Abstracts

\title{
2021 AOA Research Abstracts and Poster Competition
}

https://doi.org/10.1515/jom-2021-2000

Published online m.

This issue of the Journal of the Osteopathic Medicine (JOM) features abstracts from the posters that were presented at the 2021 Osteopathic Medical Conference and Exposition (OMED21) in the Research Track, which took place virtually on Friday, October 22 and Saturday, October 23, 2021.

This year's abstracts were organized into Basic Science, Clinical, and Health Services categories, indicated within each abstract immediately under the abstract number. Abstracts submitted by students for the poster competition (designated with "*”) were judged, and the first- and second-place winners are designated with “ $\star$ ”)."

To enhance the readability of this special feature, abstracts have been edited for basic style only. The content has not been modified; the information provided reflects information that was submitted by the primary author, including professional degrees and affiliations.

Neither the AOA's Bureau of Osteopathic Research and Public Health Education nor the JOM assumes responsibility for the content of these abstracts.

Poster No.: *B1

Abstract No.: 4

Category: Basic Science

Research Focus Area: Chronic Diseases \& Conditions, AOA Grant Award: 19057971539

\section{Spinal ankylosis in ERAP1-/- mice is independent of IL-10}

${ }^{1}$ Patrick O’Connell, OMS-IV, ${ }^{2}$ Maja K. Blake, ${ }^{2}$ Sarah Godbehere, ${ }^{2}$ Yasser A. Aldhamen, ${ }^{3}$ Andrea Amalfitano, DO, $\mathrm{PhD}$

${ }^{1}$ Michigan State University College of Osteopathic Medicine (MSUCOM); ${ }^{2}$ Department of Microbiology and Molecular Genetics, Michigan State University College of Osteopathic Medicine (MSUCOM); ${ }^{3}$ Department of Microbiology and Molecular Genetics; Department of Pediatrics, Michigan State University College of Osteopathic Medicine (MSUCOM)

Context: The endoplasmic reticulum aminopeptidase 1 (ERAP1) gene has been linked to numerous autoimmune diseases, yet we still do not fully understand the mechanistic underpinnings of these associations. The autoimmune disease ankylosing spondylitis (AS) is the disease most strongly linked to ERAP1 single nucleotide polymorphisms (SNPs) and we recently reported that mice lacking ERAP1 (ERAP1-/-) display many of the hallmark skeletal manifestations found in human AS patients.

Objective: We also found that ERAP1-/- mice lack a particular type of immune suppressive cell called type 1 regulatory $\mathrm{T}$ cells ( $\operatorname{Tr} 1$ cells), which dampen immune responses via production of high levels of the anti-inflammatory cytokine, IL-10. Since AS patients are known to have high levels of inflammation in their spine and spinal joints, we theorized that ERAP1-mediated decreases in Tr1 cells in ERAP1-/- mice may be the cause of the dysregulated bone morphology we observe.

\section{Methods:}

Objective: Here, we aimed to confirm our previously published findings of decreased Tr1 cells in ERAP1-/- mice using more sophisticated IL-10GFP reporter mice. We also performed in vitro studies to determine if the lack of $\operatorname{Tr} 1$ cells in ERAP1-/- mice was due to a defect intrinsic to CD4+ $\mathrm{T}$ cells, or from a defect in antigen presenting cells (APCs; responsible for inducing naive CD4+ T cells into Tr1 cells). Finally, we sought to determine if the defining effector molecule of Tr1 cells, IL-10, was necessary for the prevention of spinal ankylosis in our mouse model.

Methods: We crossed our ERAP1-/- mice onto an IL-10GFP background, performed in vivo stimulation with an anti-CD3 antibody to induce $\operatorname{Tr} 1$ cell differentiation, and assessed intra-epithelial (IEC) Tr1 cells in the intestines of mice using flow cytometry. We also assessed the frequency of classical FoxP3+ Tregs in the same. Furthermore, we co-cultured WT or ERAP1-/- naive CD4+ T cells with WT or ERAP1-/- APCs to determine if lack of ERAP1 was resulting in an intrinsic or extrinsic defect in Tr1 induction. Finally, we assessed spinal bone morphology using high-resolution uCT scanning of WT and IL-10-/- mice, along with 3D reconstruction, to examine the role of IL-10 in spinal ankylosis.

Flow cytometry data was analyzed with FlowJo V10.6. uCT scans were calibrated with a phantom standard and isosurface images were created using an averaged auto-threshold of 1009 using the MicroView software. Spinal ankylosis was determined as previously described (Pepelyayeva, Y. et al. 
Scientific Reports, 2018). All statistical analyses were performed in GraphPad Prism V8, and groups were compared with a two-sided, unpaired t-test. AS is a chronic, often debilitating disease that affects individuals early in their life and primary affects the musculoskeletal system. Therefore, developing a deeper understanding of its pathogenesis aligns with the AOA chronic disease research focus area.

Results: We found that, following I.P. administration of an anti-CD3 antibody, ERAP1-/- mice had significantly less IL-10GFP+ CD4+ CD11b- Tr1 cells in the intestine compared to WT mice ( $\mathrm{p}=0.0115)$. The frequency of FoxP3+ CD4+ Treg cells in the intestines of these mice was the same. Together, this confirms our previously published findings, that lack of ERAP1 results in a specific decrease in Tr1 cells and not other regulatory CD4+ T cells. Co-culturing WT naive CD4+ T cells with WT or ERAP1-/- APCs resulted in equivalent Tr1 cell induction (mean 25\%). Co-culturing ERAP1-/- naive CD4+ T cells with WT or ERAP1-/- APCs also resulted in equivalent Tr1 induction (mean 15\%). Together, this indicates that the cause of decreased numbers of Tr1 cells in ERAP1-/- mice may be intrinsic to CD4+ T cells. Finally, we compared isosurface reconstructions from high-resolution uCT scans of WT, ERAP1-/-, and IL-10-/- mice to determine if IL-10-/- mice also have spinal ankylosis similar to our ERAP1-/- mice. As $\operatorname{Tr} 1$ cells function primarily via production of high levels of IL-10, finding a similar bone phenotype in IL-10-/- mice would support the theory that an ERAP1-mediated decrease in $\operatorname{Tr} 1$ cells is responsible for the AS-like phenotype we observe in our mouse model. However, we did not observe any spinal ankylosis in our IL-10-/- mice, suggesting that either: Tr1 cells are not responsible for the bone phenotype in ERAP1-/- mice or that $\operatorname{Tr} 1$ cells are modulating spinal ankylosis in ERAP1-/mice through an IL-10-independent mechanism.

Conclusion: Our work here confirms much of our group's previously published work, further supporting the use of ERAP1-/- mice as a powerful murine model to study AS, while also bringing to light complex mechanisms of pathogenesis that underly this disease. In particular, the apparent intrinsic deficit in CD4+ T cells caused as a result of lack of ERAP1 expression supports previous studies from our group demonstrating that ERAP1 has multiple non-canonical roles across different aspects of the immune system, apart from its wellstudied role in antigen presentation. How ERAP1 is able to intrinsically prevent naive CD4+ $\mathrm{T}$ cells from differentiating into Tr1 cells remains to be seen and is an exciting area of future investigation. The surprising finding that mice lacking IL-10 do not have spinal ankylosis makes the dependence on $\operatorname{Tr} 1$ cells more difficult to confirm. It implies that the decreased $\operatorname{Tr} 1$ cells in ERAP1-/- mice is either a side effect of global immune modulation by ERAP1, or implicates an IL-10 independent $\operatorname{Tr} 1$ cell mechanism; an interesting avenue for future exploration. Future studies on how ERAP1 controls Tr1 cells may well lead to novel treatment approaches for the many individuals affected by ERAP1-linked autoimmune diseases, since $\operatorname{Tr} 1$ cells are already being investigated in clinical trials.

References N/A

Financial Disclosures: None reported.

Support: A.A. is supported by the Osteopathic Heritage Foundation. P.O. is supported by the John A. Penner Endowed Research Fellowship, a MSU CMB student grant, and AOA student grant \#: 19057971539. Funding was used to purchase and breed mice, purchase lab supplies, general lab upkeep, purchasing reagents, purchasing time on core equipment, and to fund salaries of researchers.

Ethical Approval: All animal procedures were approved by the Michigan State University Institutional Animal Care and Use Committee (http://iacuc.msu.edu/).

Informed Consent: N/A

Poster No.: *B2

Abstract No.: 6

Category: Basic Science

Research Focus Area: Chronic Diseases \& Conditions

ERAP1 modulates proinflammatory cytokine and chemokine responses of macrophages via ER stress and inflammasome pathways

${ }^{1}$ Maja Blake, OMS-III/G3; ${ }^{2}$ Patrick O'Connell, OMS-IV; ${ }^{2}$ Sarah Godbehere; ${ }^{2}$ Yasser A. Aldhamen, PhD; ${ }^{3}$ Andrea Amalfitano, DO, PhD

${ }^{1}$ Michigan State University College of Osteopathic Medicine (MSUCOM); ${ }^{2}$ Department of Microbiology and Molecular Genetics, Michigan State University College of Osteopathic Medicine (MSUCOM); ${ }^{3}$ College of Osteopathic Medicine, Michigan State University College of Osteopathic Medicine (MSUCOM)

Context: ERAP1 gene polymorphisms have been linked to several chronic autoimmune diseases that affect the musculoskeletal system. ${ }^{1-7}$ Our lab previously published that loss of ERAP1 gene functions resulted in skeletal ankylosis and exaggerated proinflammatory immune responses in mice ${ }^{8}$, mimicking a phenotype similar to the human condition ankylosing spondylitis; however, the exact mechanism underlying these findings remained undefined.

Objective: We hypothesize that ERAP deficient mice have heightened pro-inflammatory signaling due to exaggerated ER stress (caused by loss of ERAP1 activity in the ER), and that these mechanisms are responsible for the skeletal and pro-inflammatory immune phenotypes noted in ERAP1 
deficient mice. Here, we investigated the role of ERAP1 in ER stress and inflammasome responses using ERAP1 deficient bone marrow-derived macrophages (BMDMs).

Methods: Bone marrow cells were extracted from the femurs and tibiae of male 6-8 week old WT and ERAP1-/- mice and cultured in Dulbecco modified Eagle medium (DMEM) supplemented with $10 \%$ fetal bovine serum (FBS) and 30\% supernatant derived from confluent L929 cell cultures. This procedure yields a pure population of macrophage colony-stimulating factor-dependent, adherent macrophages. BMDMs were then incubated with immune system agonists, and their cytokine responses were quantified by Bioplex, as well flow cytometry to analyze various cell surface markers of immune activation. Quantitative RT-PCR determined expression levels of ER stress genes in the WT and ERAP-/- treated BMDMs. To induce intestinal inflammation, a model of inflammatory bowel disease, also associated with several ERAP1 linked autoimmune disease patients, mice were treated with $3 \%$ dextran sodium sulfate (DSS) for 7 days. Colon homogenates from naïve and DSS-treated mice were analyzed via a multiplex-based assay to determine 23 different cytokine and chemokine concentrations.

Results: We found that exposure of ERAP1-/- BMDMs to multiple inflammasome agonists resulted in increased production of inflammasome dependent cytokines including IL-1 $\beta$, IL-18 and augmented caspase- 1 activity, as compared to WT BMDMs. These responses were associated with increased expression of ER stress-associated genes in ERAP1-/- BMDMs. Moreover, utilizing DSS as an innate immune stimulus to activate TLR and inflammasome pathways, resulted in increased levels of several proinflammatory cytokines and chemokines in the colon of ERAP1-/- mice.

Conclusion: Our results imply that ERAP1 functions are necessary to prevent excessive inflammasome activation, and suggest a model in which increased ER stress due to loss of critical ERAP1 functions correlates with induction of abnormal innate immune responses. Specifically, ERAP1 functions appear to be necessary to regulate the activation of multiple inflammasome pathways, a finding that may also explain the genetic linkage of ERAP1 polymorphisms with several human autoimmune diseases. The proinflammatory and ER stress response pathways identified here may also prove to be potential targets for autoimmune disease treatment in the future.

\section{References}

1. Popa OM, Cherciu M, Cherciu LI, et al. ERAP1 and ERAP2 Gene Variations Influence the Risk of Psoriatic Arthritis in Romanian Population. Arch Immunol Ther Exp (Warsz). 2016;64(Suppl 1):123129. doi:10.1007/s00005-016-0444-4
2. Reveille JD. Genetics of spondyloarthritis - Beyond the MHC. Nat Rev Rheumatol. 2012. doi:10.1038/nrrheum.2012.41

3. Harvey D, Pointon JJ, Evans DM, et al. Investigating the genetic association between ERAP1 and ankylosing spondylitis. Hum Mol Genet. 2009;18(21):4204-4212. doi:10.1093/hmg/ddp371

4. Reeves E, Elliott T, James E, Edwards CJ. ERAP1 in the pathogenesis of ankylosing spondylitis. Immunol Res. 2014. doi:10.1007/ s12026-014-8576-2

5. McGovern DPB, Gardet A, Törkvist L, et al. Genome-wide association identifies multiple ulcerative colitis susceptibility loci. Nat Genet. 2010;42(4):332-337. doi:10.1038/ng.549

6. Kirino Y, Bertsias G, Ishigatsubo Y, et al. Genome-wide association analysis identifies new susceptibility loci for Behçet's disease and epistasis between HLA-B ${ }^{\star} 51$ and ERAP1. Nat Genet. 2013. doi:10. 1038/ng.2520

7. Burton PR, Clayton DG, Cardon LR, et al. Association scan of 14,500 nonsynonymous SNPs in four diseases identifies autoimmunity variants. Nat Genet. 2007. doi:10.1038/ng.2007.17

8. Pepelyayeva Y, Rastall DPW, Aldhamen YA, et al. ERAP1 deficient mice have reduced Type 1 regulatory T cells and develop skeletal and intestinal features of Ankylosing Spondylitis. Sci Rep. 2018;8(1). doi:10.1038/s41598-018-30159-5

Financial Disclosures: None reported.

Support: This work was completed with funds from Dr. Andrea Amalfitano's National Institute of Health [5R01AR056981] grant, the Michigan State University Foundation, as well the Osteopathic Heritage Foundation. Funds were used to purchase reagents used in experiments. Ethical Approval: This IACUC number for this study is PROTO201900047. The review process included researchers submitting the protocol, which includes all information on experiments and animal usage, then the IACUC reviews it as a committee. Researchers then are able to make any changes needed for approval. The protocols are approved for 3 years, with any amendments during that time going to IACUC to approve. After 3 years, researchers have to submit a new protocol, even if the experiments are the same.

Informed Consent: N/A

Poster No.: ${ }^{\star} \mathrm{B} 3$

Abstract No.: 11

Category: Basic Science

Research Focus Area: Impact of OMM \& OMT

Effect of Self-Performed Osteopathic Manipulative Treatment (suboccipital release) on Heart Rate Recovery: A Pilot Study

${ }^{1}$ Dylan Charles Loquist MS, OMS-I; ${ }^{2}$ Ted Wong, PhD; ${ }^{3}$ Michael Warner, DO, CPC, CPCO, CPMA, AAPC Fellow; 
${ }^{4}$ Sallie Cannumay, MS, OMS-I; ${ }^{4}$ Gurmun Singh, MS; ${ }^{4}$ Dante Nazarian, MS; ${ }^{4}$ Nitin Saukhla, MS; ${ }^{4}$ Tyler Hovsepian, MS

${ }^{1}$ Touro University College of Osteopathic Medicine-CA (TUCOM); ${ }^{2}$ Department of Basic Science, Touro University College of Osteopathic Medicine-CA (TUCOM); ${ }^{3}$ Department of Osteopathic Manipulative Medicine, Touro University College of Osteopathic Medicine-CA (TUCOM); ${ }^{4}$ Touro University College of Osteopathic Medicine-CA (TUCOM)

Context: A rapid heart rate recovery (HRR) correlates with cardiovascular health and a slower HRR with cardiac mortality. It is known that improving HRR via exercise reduces mortality in patients during cardiac rehab after a heart procedure. However, recovering patients often have difficulty performing rigorous physical activity. Thus, identifying interventions such as OMT capable of causing a more rapid HRR is a clinically important area of investigation.

Objective: 1. Examine how suboccipital release OMT influences autonomic balance via parallel changes in heart rate variability (HRV). 2. Determine the effectiveness of suboccipital release OMT to facilitate post-exercise heart rate recovery (HRR). Hypothesis: Self-administered OMT performed after a single bout of high-intensity interval training (HIIT) causes a more rapid HRR that correlates with increased parasympathetic tone during the post-exercise period.

Methods: Study utilized a prospective intervention study design. Subjects were recruited from a cohort of TUC students. Only volunteers deemed able to safely complete all study protocols based on health questionnaire responses were selected based on Inclusion/Exclusion criteria. 12 subjects were recruited and 9 successfully completed the protocol.

Subjects were trained in self-administration of the suboccipital release OMT technique by instructional video based on protocols utilized for training osteopathic students at Touro University California. Subjects also trained in the use of phone apps used to monitor HR variability (HRV) and HR recovery (HRR) (EliteHRV®; Polar Beat $\left.{ }^{\circledR}\right)$. Proficiency was supervised and facilitated by student researchers trained by principle investigators. Baseline (resting) HRV and HRR parameters for each subject were determined for later comparison to exercise measurements.

Using a repeated-measures design, subjects completed two 20-minute sessions of high-intensity interval training (HIIT) bouts followed by a 5 min recovery period: Session 1: OMM Recovery (occipital release performed) and Session 2 Passive Recovery (quite rest) during 5 min recovery period. Sessions were performed 48 hours apart and subjects were randomized in the order of session performance. HRV and HRR data were monitored throughout the exercise and recovery periods via smartphone applications synched to a heart rate chest monitor (Polar H10 $\left.{ }^{\circledR}\right)$. HRV and HRR data were stored on online platforms for later analysis.

Group HRV and HRR data from each exercise session were analyzed statistically for differences by T-test with an alpha level of 0.05

Improving HRR via exercise is known to reduce mortality in patients recovering from heart procedures. However, recovering patients have difficulty performing rigorous physical activity. Thus, identifying interventions such as Osteopathic Manipulative Treatment (OMT) capable of causing a more rapid HRR is osteopathically significant.

Results: Due to user error/incomplete results from the data collection app EliteHRV®, the sample size for HRV data was 5. Each index for HRV displayed a significantly higher HRV for the OMT recovery group compared to the no OMT recovery group. HRV data was analyzed as HRV Frequency Domain Power (FDP). Units for FDP are displayed as $\mathrm{ms}^{2}$.

The major findings from this data included significantly ( $<<0.05)$ higher HRV with the OMT Recovery vs. the Passive Recovery for the Low-Frequency (LF) domain and Total Power (TP) domain of HRV indices. The closer the HRV is to the Baseline Readiness value, the larger the HRV.

Legend: (OMT recovery; no OMT recovery; Readiness Baseline Value)

LF: $1928.83 \mathrm{~ms}^{2} ; 220.95 \mathrm{~ms}^{2} ; 2656.06 \mathrm{~ms}^{2}$

TP: $2801.19 \mathrm{~ms}^{2} ; 362.14 \mathrm{~ms}^{2} ; 3460.77 \mathrm{~ms}^{2}$

Statistically significant differences in each of the indices related to the HRV for OMT recovery were found when compared to the no OMT recovery data. All data were analyzed utilizing a t-test with a significance level of $0.05(\alpha=0.05)$.

Due to user error/incomplete results from the data collection app Polar Beat ${ }^{\circledR}$, the sample size for HRR data was 5 for the OMT recovery group data and 7 for the no OMT recovery group data. Results displayed a greater HRR for the no OMT recovery group at both one-minute post-exercise (28.4 HRR for OMT Recovery vs. 40.8 HRR for no OMT Recovery) and five minutes post-exercise (64 HRR for OMT Recovery vs. 63.7 HRR for OMT Recovery). These results are not statistically significant, and no statistical testing was able to be performed due to incomplete HRR results from issues with data collection through the HRR applications used.

Conclusion: The HRV group data was consistent with our hypothesis and previous literature, as it showed that the self-administered OMT increases certain HRV variables that correspond to increased parasympathetic tone (LF and TP). The HRR group data showed no benefit from the OMT recovery, as the no OMT recovery group had larger HRRs at one minute and five minutes post-cessation of exercise. These findings are paradoxical in that it is known HRR is predominantly a function of the reactivation of the parasympathetic nervous system, thus vagal activity. 
The paradox in our findings regarding HRR data could be possibly explained by general study limitations, limited sample size, user error on data collecting applications, and failure to create separate HRV/HRR readings during the recovery phase of the HIIT sessions.

Our results suggest that untrained individuals can perform self-administered OMT (suboccipital release) and reap the physiological benefit of an improved HRV (and in theory improved HRR). Further research is required to make a statistically significant claim regarding the extent of the benefit self-administered OMT (suboccipital release) may have on HRR/HRV.

\section{References}

1. McArdle, William. Katch, Frank. Katch, Victor. Exercise Physiology. Fourth Edition. Baltimore, Maryland: Lippincott Williams \& Wilkins, 1996.

2. Qiu, S., Cai, X., Sun, Z., Li, L., Zuegel, M., Steinacker, J. M., Schumann, U. Heart Rate Recovery and Risk of Cardiovascular Events and All-Cause Mortality: A Meta-Analysis of Prospective Cohort Studies. Journal of the American Heart Association Volume 6, Issue 5, 9 May 2017

3. Arai Y, Saul JP, Albrecht P, et al. Modulation of cardiac autonomic activity during and immediately after exercise. Am J Physiol 1989;256:H132-H141

4. Imai $\mathrm{K}$, Sato $\mathrm{H}$, Hori $\mathrm{M}$, et al. Vagally mediated heart rate recovery after exercise is accelerated in athletes but blunted in patients with chronic heart failure. J Am Coll Cardiol 1994;24:1529-1535

5. Jolly, M. A., Brennan, D. M., Leslie Cho, L. Impact of Exercise on Heart Rate Recovery. Circulation Volume 124, Issue 14, 4 October 2011, Pages 1520-1526

6. HeartMath Institute (Ed.). (n.d.). Chapter 03: Heart Rate Variability. Retrieved February 17, 2020, from https://www.heartmath.org/ research/science-of-the-heart/heart-rate-variability/

7. Shaffer, F., \& Ginsberg, J. P. (2017). An Overview of Heart Rate Variability Metrics and Norms. Frontiers in Public Health, 5. doi: 10.3389/fpubh.2017.00258

8. Giles, P. D., MS, Hensel, K., Pacchia C. F., and Smith, M.L. Suboccipital Decompression Enhances Heart Rate Variability Indices of Cardiac Control in Healthy Subjects. The Journal of Alternative and Complementary Medicine Volume 19, Number 2, 2013, pp. 92-96

9. Cuoco, J. A., Fennie, C. N., and Cheriyan, G. K. Hypothetical Link Between Osteopathic Suboccipital Decompression and Neuroimmunomodulation. J. Neurology and Neuroscience Vol.7 No.S3:133

10. Atkins, D.V. and Eichler, D. A. The Effects of Self-Massageon Osteoarthritis of the Knee: a Randomized, Controlled Trial. International Journal of Therapeutic Massage and BodyworkVolume 6, Number 1, March 2013

11. American College of Sports Medicine (ACSM) Information of High Intensity Interval Training (https://www.acsm.org/docs/defaultsource/files-for-resource-library/high-intensity-intervaltraining.pdf?sfvrsn=b0f72be6_2)
Financial Disclosures: None reported.

Support: None reported.

Ethical Approval: Approved by Expedited Review process by Touro University-California Institutional Review Board (TUC IRB FWA00009823: expiration 10/24/2024; IRB00004515; IORG0003813: expiration 10/15/2022). TUC IRB Study \# M-1221 (Approval Period 4/27/2021 - 4/27/2022

Informed Consent: Healthy males and females over the age of 18 years were recruited for the study on a volunteer basis from students enrolled in the Touro UniversityCalifornia (TUC) Master of Science in Medical Health Science (MSMHS) Class of 2021. A MSMHS 2021 class listserv and class-specific social media platforms were used for this purpose. Individuals interested in participating as subjects were provided a copy of the Consent Form to Participate as a Human Research Study to review. Consent was then provided by signing and returning the consent form, subjects were then screened via a Pre-Participation Questionnaire to determine if they met the subject eligibility and safety criteria. Questions were designed to assess 1 General Health and 2 Exercise Tolerance. Questionnaires were evaluated by the principle investigator and only healthy volunteers deemed able to safely complete all study protocols were selected based on specific Inclusion and Exclusion Criteria.

Poster No.: ${ }^{\star} \mathrm{B} 4$

Abstract No.: 14

Category: Basic Science

Research Focus Area: Chronic Diseases \& Conditions

EGR2 deletion decreases immunoglobulin production in lupus mice

${ }^{1}$ Joseph Raymond Spengler, OMS-II, MS; ${ }^{2}$ Rujuan Dai, PhD; ${ }^{3}$ Chris Reilly, $\mathrm{PhD}$

${ }^{1}$ Edward Via College of Osteopathic Medicine (Virginia); ${ }^{2}$ Department of Biomedical Sciences and Pathobiology, VA-MD College of Veterinary Medicine; ${ }^{3}$ Department of Cellular Biology and Physiology, Edward Via College of Osteopathic Medicine (Virginia)

Context: Autoimmune diseases, such as systemic lupus erythematosus, are described as auto reactive inflammatory responses where the immune system mistakenly attacks healthy body cells. When inherent immunoregulatory mechanisms are lost, the disease can progress. Early growth response gene 2 (EGR2) is highly upregulated in human and murine lupus cells. We have previously shown that EGR2 
expression induced Th1 differentiation and IFNy production in lupus effector CD4+ T cells.

Objective: In our present studies we sought to determine the role of EGR2 on B cell antibody production.

Methods: Naïve B-Cell were isolated from floxed EGR2 B6/ lpr mice (lupus mice) and from EGR2-/- B6/lpr mice, and then cultured in vitro with no stimulation (control), LPS, antiCD40, or anti-IgM for 5 days. IgG and IgM production were then measured using ELISA on cell culture supernatant.

Results: When compared to controls we found an increase in IgG production cultured B cells stimulated with antiCD40, LPS and anti-IgM. IgG production was decreased in anti-IgM stimulated B cells from EGR2-/- B6/lpr mice compared to wildtype. However, compared with control, there was no difference in IgM production among knock out and wildtype B cells stimulated with LPS and antiCD40. IgM production was decreased in both knock out and wildtype B cells stimulated with anti-IgM.

Conclusion: Our data suggests that EGR2 may play a role in antibody production in lupus, and that blocking EGR2-/- may decrease autoantibody production in lupus.

\section{References}

1. Dai R, Heid B, Xu X, Xie H, Reilly CM, Ahmed SA. EGR2 is elevated and positively regulates inflammatory IFN $\gamma$ production in lupus CD4+ T cells. BMC Immunol. 2020 Jul 9;21(1):41. doi: 10.1186/ s12865-020-00370-z. PMID: 32646370; PMCID: PMC7346656.

Financial Disclosures: None reported.

Support: One Health Grant, Internal Funds.

Ethical Approval: Institutional Animal Care and Use Committee (IACUC) approval

Informed Consent: N/A

Poster No.: B5

Abstract No.: 15

Category: Basic Science

Research Focus Area: Chronic Diseases \& Conditions

\section{Heavy Metal Exposure in Rice and its Impact on Infant and Child Health Worldwide}

\author{
${ }^{1}$ Tracee Guthrie, OMS-I; ${ }^{2}$ Brooke Benjamin; ${ }^{3}$ Gurleen Kaur; \\ ${ }^{3}$ Erin Collins
}

${ }^{1}$ Edward Via College of Osteopathic Medicine (Virginia); ${ }^{2}$ Department of Research, Kentucky College of Osteopathic
Medicine; ${ }^{3}$ Department of Microbiology and Immunology, Edward Via College of Osteopathic Medicine (Virginia)

Context: Heavy metals in particular cadmium, arsenic, and lead have developed into a prominent topic as potential sources of toxicity. This paper aims to highlight the key aspects of exposure, distribution, and metabolism of arsenic, cadmium, and lead with an emphasis on rice as a source of exposure. Additionally, this paper highlight the need for improved public awareness and education for prevention of exposure to heavy metals via rice ingestion. Objective: This paper aims to highlight the key aspects of exposure, distribution, and metabolism of the heavy metals arsenic, cadmium, and lead with an emphasis on rice as a source of exposure in vulnerable populations like pregnant and breastfeeding mothers.

Methods: 1. Rice samples grown in China, Thailand, and India, were purchased from a variety of Asian grocery stores throughout Blacksburg and Roanoke, Virginia (Zip codes 24060 and 24018).

2. Rice from each sample purchased was removed and 250 cubic centimeters placed in a plastic sample bag with its identifying collection number with identifying information recorded for each purchased sample of rice (Table 1).

3. All rice samples collected were identified as to purchase location, country of origin, exporter/importer information, whether rice was polished (white rice) or unpolished (brown rice). Controls for the experiment consisted of five samples of rice grown in the United States.

4. All rice samples were sent to the Analytical Research Laboratory, Virginia-Maryland College of Veterinary Medicine, Blacksburg, Virginia 24060, for analysis using Inductively Coupled Plasma with Mass Spectrometry Detection (ICP-MS) Analysis of Total Metals in Rice.

5. Representative portions of rice samples were microwave digested in modified polytetrafluoroethylene (TFM-PTFE) vessels using a solution mixture of trace metal grades of nitric acid (HNO3) and hydrogen peroxide (H2O2) to ensure complete digestion of the rice matrix. The resulting clear digests were then diluted in $2 \%(w / v) \mathrm{HNO}$ $+0.5 \% \mathrm{HCl}$ prior to ICP-MS analysis.

6. Metal content was quantified using a calibration curve with known concentrations of mercury in the same dilute acid diluent as the samples.

7. ICP-MS analysis of these standards and samples was performed by directly monitoring several metal isotopes at 199, 200, 201, and $202 \mathrm{~m} / \mathrm{z}$.

8. In addition to multiple isotope monitoring, the use of a helium collision cell were further minimize and/or to eliminate potential polyatomic interferences and an on-line addition internal standard solution comprised of bismuth 
( $209 \mathrm{~m} / \mathrm{z}$ ) was utilized to correct for any potential instrumental response variations observed during the analysis.

Results: Each of the 29 rice samples collected were analyzed for $\mathrm{As}, \mathrm{Cd}$, and $\mathrm{Pb}$ using inductively coupled plasma mass spectrometry (ICP-MS). The concentration for each sample was represented as ng of element/g of sample. When concerning As, USA and China were in the top 5 samples with the highest levels of As. With the USA sample (Sample ID: 143-1) having the highest level of As, with $263 \mathrm{ng}$ of element/ $\mathrm{g}$ of sample. When concerning Cd, the sample from China (Sample ID: 143-27) had the highest level of Cd, with $165.6 \mathrm{ng}$ of element/ $\mathrm{g}$ of sample. The USA, China, India and Thailand were all in the top 5 samples with the highest level of $\mathrm{Cd}$. The highest level of $\mathrm{Pb}$, came from China (Sample ID: 143-27) with $114.65 \mathrm{ng}$ of element/g of sample. In the top 5 samples with the highest levels of $\mathrm{Pb}$ were India, China, and Thailand. The red color represents the amount which is below the level of quantitation.

Conclusion: These worries of heavy metal exposure are further highlighted by the data collected. The data in table 1 , illustrates that when concerning Arsenic concentrations, that the United States (control) did not differ in concentrations when compared to those samples grown in Thailand, India, and China. The same can be said for the data in table 2, concerning Cadmium, where the United states samples did not differ in concentrations when compared to those samples grown in Thailand, India, and China. The data from table 3, highlights that the United States (control) varied in concentrations when concerning Lead. The samples which were collected and manufactured in the United States served as the control, and was hypothesized that these would not have high levels of heavy metals. But, the data highlights that from all the samples collected that the United States was in the top ten, especially when concerning Cadmium and Arsenic. It is possible that the United States has a variety of other contributing factors which would cause high levels of heavy metals similar to those samples of rice produced in other countries. Additionally, the samples which were grown and manufactured in China showed high levels of $\mathrm{As}, \mathrm{Cd}$, and $\mathrm{Pb}$ and were in the top ten for each.

Viable resolutions for preventing exposure to these heavy metals include performing more research, providing public education, and expanding regulations for heavy metal content in rice. There is a need to assess more closely what can be done to restrict how heavy metal content becomes incorporated into the rice. Moreover, public education is necessary so that informed decisions can be made prior to eating rice and possibly decrease exposure, especially when concerning infants, expecting and breastfeeding mothers. Furthermore, increasing regulations for rice production needs to be considered.

\section{References}

1. Kukusamude C, Sricharoen P, Limchoowong N, Kongsri S. Heavy metals and probabilistic risk assessment via rice consumption in Thailand. Food Chemistry. 2021;334:127402. doi:10.1016/j. foodchem.2020.127402

2. Zhang Z, Zhang N, Li H, Lu Y, Yang Z. Potential health risk assessment for inhabitants posed by heavy metals in rice in Zijiang River basin, Hunan Province, China. Environmental Science and Pollution Research. 2020;27(19):24013-24024. doi:10.1007/ s11356-020-08568-9

3. Pateriya A, Kumar R, Singh Sankhla M, Kumar Verma R. Heavy Metal Toxicity in Rice and its Effects on Human Health. Letters in Applied NanoBioScience. 2020;10(1):1833-1845. doi:10.33263/ lianbs101.18331845

4. Rothenberg SE, Jackson BP, Carly McCalla G, Donohue A, Emmons AM. Co-exposure to methylmercury and inorganic arsenic in baby rice cereals and rice-containing teething biscuits. Environmental Research. 2017;159:639-647. doi:10.1016/j. envres.2017.08.046

5. Naujokas MF, Anderson B, Ahsan H, et al. The Broad Scope of Health Effects from Chronic Arsenic Exposure: Update on a Worldwide Public Health Problem. Environmental Health Perspectives. 2013;121(3):295-302. doi:10.1289/ehp.1205875

6. Davis MA, Signes-Pastor AJ, Argos M, et al. Assessment of human dietary exposure to arsenic through rice. Science of The Total Environment. 2017;586:1237-1244. doi:10.1016/j.scitotenv.2017. 02.119

7. Zwolak I. The Role of Selenium in Arsenic and Cadmium Toxicity: an Updated Review of Scientific Literature. Biological Trace Element Research. 2019;193(1):44-63. doi:10.1007/s12011-019. 01691-w

8. Falcó G, Llobet JM, Bocio A, Domingo JL. Daily Intake of Arsenic, Cadmium, Mercury, and Lead by Consumption of Edible Marine Species. Journal of Agricultural and Food Chemistry. 2006; 54(16):6106-6112. doi:10.1021/jf0610110

9. Rolston DDK. Uncommon Sources and Some Unsual Manifestations of Lead Poisoning in a Tropical Developing Country. Tropical Medicine and Health. 2011;39(4):127-132. doi:10.2149/tmh. 2011-01

10. Mao C, Song Y, Chen L, et al. Human health risks of heavy metals in paddy rice based on transfer characteristics of heavy metals from soil to rice. CATENA. 2019;175:339-348. doi:10.1016/j. catena.2018.12.029

Financial Disclosures: None reported.

Support: None reported.

Ethical Approval: The study was deemed exempt. Informed Consent: Not relevant. 
Poster No.: B6

Abstract No.: 18

Category: Basic Science

Research Focus Area: Chronic Diseases \& Conditions

Assessing CCR1 Antagonists for Chemotaxis Inhibition in a Multiple Myeloma in vitro Model

${ }^{1}$ Stephanie Lourdes Echeverria, OMS-I, MPH, MBS; ${ }^{2}$ Annette Gilchrist, $\mathrm{PhD}$

${ }^{1}$ Midwestern University Arizona College of Osteopathic Medicine (MWU/AZCOM); ${ }^{2}$ Department of Pharmaceutical Sciences, Edward Via College of Osteopathic Medicine (Virginia)

Context: Multiple myeloma (MM) is a plasma B-cell malignancy characterized by osteolytic bone lesions. ${ }^{1} \mathrm{MM}$ cells secrete CCL3/MIP1 $\alpha$, a chemokine with the ability to induce cell migration, which has been shown to induce osteoclastogenesis. ${ }^{2}$ CCR1, a G-protein coupled receptor (GPCR) activated by CCL3 is endogenously expressed on both MM cells and osteoclast precursor cells. ${ }^{3}$

Objective: Previously, we showed that human recombinant CCL3-mediated chemotaxis was inhibited in the RPMI8226 MM cell line in a dose-dependent manner by six CCR1 antagonists. Here we assess the effects of the same six CCR1 antagonists on the U266 MM cell line and U266_CCR1, which stably express hCCR1. We hypothesized the CCR1 antagonists would result in a dose-dependent inhibition of chemotaxis in both U266 and U266_CCR1 cell lines, similar to that observed with RPMI8226.

Methods: The CCR1 antagonists used for these experiments included AZD4818, BX471, CCX354, CP481715, MLN3897, P031291, and MG1-5. We then treated either the MM cells or the RAW 264.7 cells with the CCR1 antagonists and compared the results. Equivalent numbers of U266 and U266_CCR1 cells are centrifuged, and the pellet brought up in HHBS (Hank's Balanced Salt Solution/10mM HEPES). Cells are incubated with Calcein $(2 \mathrm{mM})$ at $37^{\circ} \mathrm{C}$, washed, and brought up in HHBS. Serial dilutions of CCR1 antagonists $(0.001 \mu \mathrm{M}-9.64 \mu \mathrm{M})$ are added to the cells and the mix placed into the top chamber of a 96-well Multiscreen ${ }^{\circledR}$-MIC plates (Millipore) with $5 \mu \mathrm{M}$ pore size. 20\% RAW supernatant (spn) or 20\% Fetal Bovine Serum (FBS) are placed in the bottom chamber. The chemotaxis plate is then incubated at $37^{\circ} \mathrm{C}$ for 2 hours. $50 \mu \mathrm{L} /$ well of the bottom chamber is read using EX490/EM520 on a DTX 800 multimode plate reader. On Day 1 RAW 264.7 cells at 50-70\% confluency in DMEM Starved Media are placed in designated wellsof 96-well culture plate at $90 \mu \mathrm{L} /$ well. On Day 2, serial dilutions of CCR1 antagonists $(0.001 \mu \mathrm{M}-10 \mu \mathrm{M})$ are added to the RAW cells. On Day 3, RAW spn is removed and placed in designated wells of the bottom chamber of the chemotaxis plate for data collection 24-hour post RAW cell CCR1 antagonist treatment. The RAW cells are then re-dosed in an identical manner. On Day 4, RAW spn is removed and placed in designated wells of the bottom chamber of the chemotaxis plate for data collection 48-hour post RAW cell CCR1 antagonist treatment. The MM cell lines are prepared in an identical manner as described in Aim 1 Methods, but without the treatment of CCR1 antagonists, and placed in the top chamber of the chemotaxis plate. The chemotaxis plate is then incubated at $37^{\circ} \mathrm{C}$ for 2 hours. $50 \mu \mathrm{L} /$ well of the bottom chamber is read using EX490/EM520 on a DTX 800 multimode plate reader.

Results: Each experiment was performed as three independent experiments, $\mathrm{n}=3$ with the exception of MG1-5, $\mathrm{n}=1$. Experiments for treated MM cell lines were performed in triplicate. Data for treated RAW 264.7 cells was performed in quadruplicate. Technical replicates were used to ensure the reliability of single values. Data was normalized and non-linear regression analysis was completed. Data in the figures are expressed as mean \pm SEM. All data analysis was completed with GraphPad Prism software version 9.0. No inhibition of chemotaxis was observed to RAW spn or FBS with either cell line following treatment with the CCR1 antagonists. However, when the RAW 264.7 cells were treated with the CCR1 antagonists, CCX354 decreased cell migration of both cell lines in a concentration dependent manner.

Conclusion: Given that inhibition has previously been shown with RPMI8226 cells under similar conditions for several of the compounds ${ }^{3}$, our findings highlight the challenges of working with allosteric antagonists and how contextual differences such as cell line can have profound differences. That CCX354 was uniquely able to inhibit RAW264.7 induced cell migration may be due to species differences in CCR1. There are significant differences in expression and function of CCR1 between human and animal models. Mouse models for instance show CCR1 as a chemotactic factor for neutrophils but not monocytes. Such interspecies differences can lead to the misinterpretation of results from animal disease models and limit the ability to target the appropriate chemokine receptor in a human disease model. ${ }^{4}$ CCX721, an analogue of CCX354, has been shown to block the formation of mature osteoclasts and decrease tumor burden and osteolytic damage in a mouse model of MM (5TGM1). ${ }^{5}$ Furthermore, in double-blind, placebo-controlled, single- and multiple-dose phase I studies, CCX354 was well-tolerated, displayed a linear 
dose-exposure profile, and demonstrated high levels of receptor coverage 12-hours after an initial dose. ${ }^{6}$ That CCX354 and analogues of CCX354 (CCX721) have exhibited favorable results in both murine and human models, could be the reason why CCX354 proved effective in our mixed in vitro model containing a human MM cell line and murine osteoclast precursor. CCX354, which displayed chemotaxis inhibition for both the U266 and U266_CCR1 cell lines with antagonist treated RAW 264.7 cells, could have broader efficacy and work with the murine cell line. It will be interesting to learn if this compound will behave similarly with human MM cells induced to migrate using spn from human osteoclasts. Of the antagonists examined, CCX354 may prove to be a valuable new tool in treating multiple myeloma.

\section{References}

1. Michels TC, Petersen KE. Multiple Myeloma: Diagnosis and Treatment. Am Fam Physician. 2017;95(6):373-383.

2. Menu E, De Leenheer E, De Raeve H, et al. Role of CCR1 and CCR5 in homing and growth of multiple myeloma and in the development of osteolytic lesions: a study in the 5TMM model. Clin Exp Metastasis. 2006;23(5-6):291-300. doi:10.1007/s10585-0069038-6

3. Gilchrist A, Gauntner TD, Fazzini A, et al. Identifying bias in CCR1 antagonists using radiolabelled binding, receptor internalization, $\beta$-arrestin translocation and chemotaxis assays. $\mathrm{Br} J$ Pharmacol. 2014;171(22):5127-5138. doi:10.1111/bph.12835

4. Gladue RP, Brown MF, Zwillich SH. CCR1 antagonists: what have we learned from clinical trials. Curr Top Med Chem. 2010;10(13):12681277. doi:10.2174/156802610791561237

5. Dairaghi DJ, Oyajobi BO, Gupta A, et al. CCR1 blockade reduces tumor burden and osteolysis in vivo in a mouse model of myeloma bone disease. Blood. 2012;120(7):1449-1457. doi:10.1182/blood2011-10-384784

6. Dairaghi DJ, Zhang P, Wang Y, et al. Pharmacokinetic and pharmacodynamic evaluation of the novel CCR1 antagonist CCX354 in healthy human subjects: implications for selection of clinical dose. Clin Pharmacol Ther. 2011;89(5):726-734. doi:10.1038/clpt. 2011.33

Financial Disclosures: None reported.

Support: This work was supported by the Biomedical Sciences Department, College of Graduate Studies at Midwestern University, and the Chicago College of Pharmacy (A.G.). We would like to thank Dr. Merritt for the privilege of using the CCR1 antagonists synthesized at Kean University. Ethical Approval: Deemed exempt from IRB or IACUC approval

Informed Consent: N/A
${ }^{\star}$ Poster No.: *B7

Abstract No.: 19

Category: Basic Science

Research Focus Area: Chronic Diseases \& Conditions

High Fat Diet and High-IntensityInterval Training (HIIT) on Appetite Regulation in a Rat Model

${ }^{1}$ Sarai Burke Arbus, OMS-III; ${ }^{2}$ Kelsey Scherer, OMS-III; ${ }^{2}$ John Pirtle, OMS-III; ' ${ }^{2}$ Jessica King, OMS-II; ${ }^{2}$ Shawn Plyler, OMS-III; ${ }^{2}$ Christopher L. Pankey, PhD

${ }^{1}$ West Virginia School of Osteopathic Medicine (WVSOM); ${ }^{2}$ Department of Biomedical Sciences, West Virginia School of Osteopathic Medicine (WVSOM)

Context: The growing obesity epidemic has led to an intensified focus on the maladaptation of energy homeostasis to obesity. ${ }^{1}$ Research has revealed that high fat diets stimulate overeating, positive energy balance, and weight gain. ${ }^{2}$ High-intensity-interval training has been shown to blunt hyperphagia and improve satiety, in addition to increasing energy expenditure. ${ }^{3,4,5,6}$ The aim of this study is to evaluate the effects of high fat diet and high-intensityinterval training on appetite regulation.

Objective: To investigate the effects of a high fat diet and high-intensity-interval-training (HITT) on appetite regulation in a rat model.

Methods: Thirty-nine female rats were randomly assigned to exercise and diet protocols, resulting in 4 groups: control diet/sedentary (CONsd, $n=10$ ), control diet/exercise training (CONtr, $\mathrm{n}=10$ ), high fat diet/sedentary (HFDsd, $n=9$ ), high fat diet/exercise training (HFDtr, $n=10$ ). Control (CON) diets consisted of chow with 10\% kilocalories (kcal) from fat and high fat diets (HFD) with 45\% kcal from fat. From PND 28 to PND 84 all subjects were given ad libitum access to food and water.

Exercise training (tr) ran from PND 84 to PND 140, consisting of 4 sessions per week. For each session, rats in the same group were placed in individual lanes on a rodent treadmill. Each training session began with a 5-minute acclimation period (treadmill set to $10 \mathrm{~cm} / \mathrm{sec}$ ), followed by 10 HIIT bouts. Each bout consisted of 1 minute of running, then 2 minutes of rest. Running speed began at $55 \mathrm{~cm} / \mathrm{sec}$. Stiff bristled brushes and a shock grid (set to $2 \mathrm{~mA}$ maximum) were used to encourage forward motion. If all subjects within a cohort ran without agitation or hitting the shock grid for a full 1-minute interval, the speed was increased by $4 \mathrm{~cm} / \mathrm{sec}$ for the remaining bouts. New 
training sessions began with a speed $4 \mathrm{~cm} / \mathrm{sec}$ slower than the fastest speed obtained in the prior workout $(55 \mathrm{~cm} / \mathrm{sec}$ minimum).

Feed intake of each subject was recorded per day and analyzed using two-way analysis of variance with group (CONtr, CONsd, HFDtr, HFDsd) treated as a between group variable and time treated as a within subject variable.

Osteopathic philosophy asserts that the human body has an innate capacity to self-regulate. This self-regulation of energy intake is disrupted in high-fat diets. Unfortunately, the interplay between diet and exercise, as it relates to one's ability to self-regulate, is poorly understood. This study seeks to improve our understanding of the relationship between diet, exercise and energy homeostasis.

Results: Mean weekly caloric intake was influenced by an interactive effect of diet and exercise training. As expected, the average weekly caloric intake of rats fed a HFD was higher $(\mathrm{p}<0.0001)$ than rats fed a control diet $(430 \pm 4.6 \mathrm{vs}$ $396 \pm 3.7 \mathrm{kcal} / \mathrm{wk}$, respectively). No difference was observed in the weekly caloric intake between sedentary and exercise groups $(409 \pm 4.1$ vs. $419 \pm 5.2 \mathrm{kcal} / \mathrm{wk}$, respectively), although a trend was identified $(\mathrm{p}=0.0571)$. Weekly caloric intake was lower $(\mathrm{p}<0.05)$ in CONex than CONsd (360 \pm 5.3 vs. $402 \pm 5.4 \mathrm{kcal} / \mathrm{wk}$, respectively), but higher $(\mathrm{p}<0.05)$ in HFDex than HFDsd $(476.6 \pm 6.8$ vs. $416 \pm 6.3 \mathrm{kcal} / \mathrm{wk}$, respectively). No difference was observed in distance run between CONex and HFDex ( $54.6 \pm 0.6$ vs. $54.1 \pm 0.5 \mathrm{~m} /$ bout, respectively).

Conclusion: The goal of this study was to determine the impact of diet and high-intensity-interval training on appetite regulation in a rat model. These data indicate that subjects that consumed a high-fat diet and underwent exercise training consumed more calories than those that were sedentary and assigned a high-fat diet. In contrast, the subjects that consumed a control diet and underwent high-intensity-interval training consumed fewer calories than those that were sedentary and assigned a control diet, demonstrating an inverse response to maintaining energy homeostasis depending on diet composition. This suggests that weight loss strategies implementing a high-intensity exercise program may be blunted if individuals are consuming a high fat diet as there may be a propensity to overconsume. Conversely, exercise training coupled with a better macronutrient balance may encourage hypocaloric intake, thereby facilitating a negative energy balance. A deeper understanding of the mechanisms behind this observation are critical to developing effective weight loss strategies, and further research is needed to determine how translatable it may be to human conditions.

\section{References}

1. Timper K, Brüning JC. Hypothalamic circuits regulating appetite and energy homeostasis: pathways to obesity. Dis Model Mech. 2017;10(6):679-689. doi:10.1242/dmm.026609

2. Eckel LA, Moore SR. Diet-induced hyperphagia in the rat is influenced by sex and exercise. Am J Physiol Regul Integr Comp Physiol. 2004;287(5):R1080-5. doi:10.1152/ajpregu.00424.2004

3. Sim AY, Wallman KE, Fairchild TJ, Guelfi KJ. Effects of HighIntensity Intermittent Exercise Training on Appetite Regulation. Med Sci Sports Exerc. 2015;47(11):2441-2449. doi:10.1249/ MSS.0000000000000687

4. Steinberg GR, Smith AC, Wormald S, Malenfant P, Collier C, Dyck DJ. Endurance training partially reverses dietary-induced leptin resistance in rodent skeletal muscle. Am J Physiol Endocrinol Metab. 2004;286(1):E57-E63. doi:10.1152/ajpendo.00302.2003

5. Nance DM, Bromley B, Barnard RJ, Gorski RA. Sexually dimorphic effects of forced exercise on food intake and body weight in the rat. Physiol Behav. 1977;19(1):155-158. doi:10.1016/00319384(7790173-1)

8. Martins C, Morgan L, Truby H. A review of the effects of exercise on appetite regulation: an obesity perspective. Int J Obes (Lond). 2008;32(9):1337-1347. doi:10.1038/ijo.2008.98

Financial Disclosures: None reported.

Support: This work was supported by West Virginia School of Osteopathic Medicine internal funds.

Ethical Approval: All study procedures were reviewed and approved by the West Virginia School of Osteopathic Medicine (WVSOM) IACUC. IACUC protocol number is 2019-5.

Informed Consent: Informed consent was not relevant to this research study as it involved animal subjects only.

Poster No.: *B8

Abstract No.: 22

Category: Basic Science

Research Focus Area: Chronic Diseases \& Conditions

\section{Trp53-R172H mutant mice have abnormal hematopoiesis and gain- of-function effect after exposing to chemotherapy stress}

${ }^{1}$ William Paul Sebastian, OMS-II; ${ }^{2}$ Lauren Forchette, OMS-I; ${ }^{2}$ Christopher Butler; ${ }^{2}$ Tuoen Liu

${ }^{1}$ West Virginia School of Osteopathic Medicine (WVSOM); ${ }^{2}$ Department of Biomedical Sciences, West Virginia School of Osteopathic Medicine (WVSOM)

Context: Myeloid malignancies, comprising myelodysplastic syndromes (MDS) and acute myeloid leukemia 
(AML), are clonal diseases of hematopoietic stem or progenitor cells. ${ }^{1}$ The TP53 gene is a tumor suppressor gene and is frequently mutated in patients with MDA and AML. ${ }^{2}$ The majority of TP53 mutations in myeloid malignancies are missense mutations. One of these hotspot mutations is a G-to-A substitution at nucleotide 525 of TP53, which codes for an Arg175His (R175H) substitution. ${ }^{3}$

Objective: To fully understand the effects of TP53 R175H mutation in the blood system, we used the mutant Trp53 $\mathrm{R} 172 \mathrm{H}$ mouse model (equivalent to $\mathrm{R} 175 \mathrm{H}$ in humans) in our study. We investigated hematopoiesis and their response to hematopoietic stressors in these mice, in comparison to loss-of-function alleles (TRP53 null or heterozygous deletion mutants) mice. We hypothesized that mutant Trp53 R172H mice have abnormal effects on hematopoiesis in mice

\section{Methods:}

Mice: Mice with different genotypes (Trp53+/+ or wildtype, Trp53R172H/+, Trp53R172H/R172H, Trp53+/-, and Trp53-/-) were used in the study.

Flow cytometry: The frequency of mature blood cells and hematopoietic stem and progenitor cells (HSPCs) was measured by flow cytometry.

Bone marrow (BM) transplantation: For noncompetitive transplantations, $2 \times 106$ donor CD45.2 BM cells of indicated genotypes were injected into $11 \mathrm{~Gy}$ lethally irradiated CD45.1 recipient mice. For competitive repopulation assays, donor CD45.2 BM cells were mixed with CD45.1/ CD45.2 competitor BM at the indicated ratio and $2 \times 106$ total cells were injected into $11 \mathrm{~Gy}$ lethally irradiated CD45.1 recipient mice.

5-Fluorouracil (5-FU) treatment: 5-FU was freshly reconstituted with $\mathrm{PBS}$ at $10 \mathrm{mg} / \mathrm{ml}$ and injected intraperitoneally into mice at the indicated doses.

Statistical analysis: Statistical analysis was performed with GraphPad Prism 8 software. Data are presented as mean \pm standard deviation. Statistical significance was defined as ${ }^{\star} \mathrm{p}<0.05 ;{ }^{\star \star} \mathrm{p}<0.01 ;{ }^{\star \star \star} \mathrm{p}<0.001 ;$ ns, not significant.

\section{Results:}

(1) TRP53 R172H mutant mice have normal frequencies of bone marrow HSPCs. The frequencies of peripheral blood and bone marrow B cells, T cells, neutrophils, and monocytes were similar among genotypes. An increase in BM LSK (Lineage-cKit+Sca1+) progenitors and LSK-SLAM stem cells in Trp53+/- and Trp53-/mice compared to the Trp53+/+ mice was observed. However, the frequency of bone marrow HSPCs was similar in Trp53R172H/+ and Trp53R172H/R172H mice compared to the wild-type mice.
(2) TRP53R172H mutant mice have reduced overall survival. The median survival of Trp53-/- and Trp53R172H/ $\mathrm{R} 172 \mathrm{H}$ mice was similar. The median survival of Trp53+/- and Trp53R172H/+ mice was not significantly different from each other.

(3) Mutant TRP53R172H hematopoietic stem cells have a competitive advantage. A competitive bone marrow transplant was performed to measure the effects of mutant Trp53 on long-term stem cell function. Analysis of peripheral blood chimerism showed that hematopoietic cells harvested from Trp53+/-, Trp53-/-, Trp53R172H/+ and Trp53R172H/R172H mice had a competitive advantage over the wild-type cells, with Trp53-/- cells having the largest advantage.

(4) Mutant TRP53R172H has a gain-of-function effect following 5-FU-induced hematopoietic stress. we exposed mice to weekly 5 -FU treatment and monitored their survival for one month. All the mice harboring Trp53-/- cells were alive by the end of the month and all the Trp53+/+ mice died within 2 weeks of 5-FU injection. Mice containing Trp53R172H/+ cells were partially resistant to 5-FU exposure as only 7 out of 10 mice died with a median survival of 21 days. We also investigated the kinetics of hematopoietic recovery after the 5-FU challenge. We found that the Trp53R172H/+ mice were least affected by the 5-FU and showed the fastest and largest recovery of WBC count compared to Trp53+/+, Trp53+/- and Trp53-/- mice. This result suggested that mutant TRP53R172H confers a gain-of-function in response to 5-FU induced cytopenia.

Conclusion: This study is helpful to underline the particular genetic functions in physiological and pathological status. We found that HSPCs are not expanded in TRP53 $\mathrm{R} 172 \mathrm{H}$ mutant mice compared to wild-type mice at baseline, in contrast to Trp53-/- and Trp53+/- mice that have expanded HSPCs. However, TRP53 R172H mutant hematopoietic stem cells have a growth advantage compared to wild-type cells. TRP53 R172H confers a gain-of-function effect to hematopoietic cells following 5-FU exposure. In summary, our results showed that mutant TRP53 R172H has distinct hematological properties and responses to particular stressors, suggesting that this mutation may contribute to the poor prognosis in patients with myeloid malignancies.

This study is also significantly relevant to the tenets of osteopathic medicine. Given that the body is a unit, it is important to understand how certain environmental stressors, such as chemotherapy treatment, interact with cancer cells, and thus how the cancer cells then affect the 
entire body of patients. For this study, in particular, patients undergoing chemotherapy will experience effects not only on the targeted cancer cells but also on the rest of the body as a whole. Moreover, understanding the patients' genetic structure and function is important when trying to restore the proper functionality of the body during disease. The relationship between structure and function at the genetic level is critical when treating the patients, especially if the treatment has the potential to exacerbate the disease.

\section{References}

1. Murati A, Brecqueville M, Devillier R, et al. Myeloid malignancies: mutations, models and management. BMC Cancer. 2012, 12:304. doi: 10.1186/1471-2407-12-304.

2. Tyner JW, Tognon CE, Bottomly D, et al. Functional genomic landscape of acute myeloid leukaemia. Nature. 2018, 562(7728):526531. doi: 10.1038/s41586-018-0623-z.

3. Lindsley RC, Saber W, Mar BG, et al. Prognostic mutations in myelodysplastic syndrome after stem-cell transplantation. N Engl J Med. 2017, 376(6):536-547. doi: 10.1056/NEJMoa1611604.

Financial Disclosures: None reported.

Support: This study was partially supported by West Virginia School of Osteopathic Medicine startup fund (Dr. Liu).

Ethical Approval: Animal experiments were approved by the Institutional Animal Care and Use Committee at the Washington University in St. Louis and West Virginia School of Osteopathic Medicine.

Informed Consent: N/A

Poster No.: B9

Abstract No.: 23

Category: Basic Science

Research Focus Area: Chronic Diseases \& Conditions

AOA Grant Award: 19137759

Transcutaneous Auricular Vagus Nerve Stimulation Induces Pancreatic Secretion of C-Peptide Together with Insulin

\footnotetext{
${ }^{1}$ Harald Martin Stauss, MD, PhD; ${ }^{2}$ Erica M. Kozorosky; ${ }^{2}$ Cristina HN Lee; ${ }^{2}$ Jessica G. Lee; ${ }^{2}$ Valeria Nunez Martinez; ${ }^{2}$ Leandra E. Padayachee

${ }^{1}$ Burrell College of Osteopathic Medicine (BCOM); ${ }^{2}$ Department of Biomedical Sciences, Burrell College of Osteopathic Medicine (BCOM)
}

Context: Transcutaneous auricular vagus nerve stimulation (taVNS) prevents the development of diabetes in rats with obesity-induced diabetes. ${ }^{1}$ In contrast, blood glucose levels are not affected by cervical vagus nerve stimulation (cVNS) in patients treated with cVNS for seizure control. ${ }^{2}$ While cVNS activates afferent and efferent vagal nerve fibers, taVNS exclusively activates afferent nerve fibers. Thus, the possibility exists that taVNS - other than cVNS elicits anti-diabetic effects in humans.

Objective: The objective of this study was to investigate the effects of taVNS on glucose metabolism in generally healthy humans. Based on the finding that taVNS prevents development of diabetes in Zucker diabetic fatty rats ${ }^{1}$, the hypothesis of this study was that taVNS lowers postprandial blood glucose levels through stimulation of pancreatic insulin secretion in humans.

Methods: The study was approved by the Burrell College Institutional Review Board. Subjects $(\mathrm{n}=16)$ were recruited from the local community in Las Cruces, NM. Following informed consent, exclusion criteria (less than 18 years of age, diabetes, pregnancy, and medications affecting glucose metabolism or the autonomic nervous system) were assessed through a questionnaire. Weight, height, blood pressure (upper arm cuff), and time of last meal were recorded. Then subjects were instrumented with EKG electrodes and a finger cuff for continuous non-invasive heart rate and blood pressure monitoring to assess the impact of taVNS or sham-taVNS on autonomic tone. Following a 30-min resting period, a capillary blood sample (finger prick) was obtained to determine blood glucose concentration (ReliOn Prime Blood Glucose Monitoring System, Walmart, Bentonville, AR) and insulin, C peptide and glucagon plasma levels (Bio-Plex assay 171A7001M, Life Science, Hercules, CA). On two different days (at least 1 week apart) taVNS $(10 \mathrm{~Hz}$ and $300 \mu \mathrm{S})$ or sham-taVNS (random order) was performed for $30 \mathrm{~min}$ followed by a 30 min recovery period, after which a second blood sample was taken. Statistical analysis was conducted using the freely available WinStat software. ${ }^{3}$ Paired t-tests were used for comparisons between data obtained before and after the intervention on both study days. The ManWhitney U-test was used for comparisons between data obtained during the taVNS and the sham-taVNS protocols. Statistical significance was assumed at $\mathrm{P}<0.05$. In line with the second osteopathic tenet, we anticipated that afferent vagal nerve stimulation by taVNS activates central nervous system pathways that result in selfregulatory processes that ultimately improve insulin sensitivity and glucose metabolism, contributing to overall health maintenance. 
Results: All subjects ( $\mathrm{n}=16,13$ female, 3 male) were normotensive. Body mass index (BMI) ranged from $17 \mathrm{~kg} / \mathrm{m}^{2}$ to $44 \mathrm{~kg} / \mathrm{m}^{2}$. None of the subjects were fasted. The time since the last meal did not differ for subjects undergoing the taVNS $(2.4 \pm 0.4 \mathrm{~h}, \mathrm{n}=14)$ or sham-taVNS $(2.7 \pm 0.3 \mathrm{~h}, \mathrm{n}=12$, n.sig.) experiments. Throughout the experimental protocol, blood glucose levels tended to decline more in response to taVNS $(-8.2 \pm 2.0 \mathrm{mg} / \mathrm{dL}$, $\mathrm{n}=14)$ than in response to sham taVNS $(4.9 \pm 1.8 \mathrm{mg} / \mathrm{dL}$, $\mathrm{n}=12, \mathrm{P}=0.12)$. Glucagon and insulin responses to the two interventions did not differ significantly. In contrast, C-peptide levels declined significantly during the shamtaVNS protocol $(0.71 \pm 0.28 \mathrm{ng} / \mathrm{mL}, \mathrm{n}=8, \mathrm{P}<0.05)$, but not during the taVNS protocol $(0.41 \pm 0.71 \mathrm{ng} / \mathrm{mL}, \mathrm{n}=8$, n.sig. $)$. A negative correlation between the change in blood glucose concentration and the change in $C$ peptide plasma levels was found in response to the taVNS intervention $(\mathrm{R}=-0.91, \mathrm{P}<0.05)$ but not in response to the shamtaVNS intervention ( $\mathrm{R}=-0.01$, n.sig.), indicating that small declines in C-peptide plasma levels during the experimental protocol are associated with large decreases in blood glucose concentrations following the taVNS intervention.

Conclusion: $\mathrm{C}$-peptide is a byproduct of pancreatic insulin synthesis that is co-released together with insulin from the $\beta$-cells within the islets of Langerhans. Due to its long halflife of 30-60 min, C-peptide can be used as a surrogate measure of insulin, which has a short half-life of only 4-6 min. In participants undergoing the sham procedure, the postprandial C-peptide plasma concentration declined throughout the study duration. This decline in $\mathrm{C}$ peptide observed during the sham-taVNS intervention was prevented by taVNS. This finding suggests that taVNS maintained C-peptide levels by stimulating pancreatic secretion of C-peptide together with insulin. These findings in healthy study participants are consistent with the idea that afferent vagal nerve stimulation by taVNS may activate central nervous system pathways that initiate selfregulatory processes that improve insulin sensitivity and glucose metabolism in patients with type 2 diabetes.

\section{References}

1. Wang S, Zhai X, Li S, McCabe MF, Wang X, Rong P. Transcutaneous vagus nerve stimulation induces tidal melatonin secretion and has an antidiabetic effect in Zucker fatty rats. PLoS One. 2015;10(4):e0124195. doi: 10.1371/journal.pone.0124195.

2. Stauss HM, Daman LM, Rohlf MM, Sainju RK. Effect of vagus nerve stimulation on blood glucose concentration in epilepsy patients Importance of stimulation parameters. Physiol Rep. 2019;7(14):110. doi: $10.14814 /$ phy2.14169.
3. Stauss HM. HemoLab Software. http://www.haraldstauss.com/ HaraldStaussScientific/hemolab.

Financial Disclosures: None reported.

Support: This study was supported by the Office of Research and Sponsored Programs at Burrell College of Osteopathic Medicine, Las Cruces, NM. Support included faculty-protected time, financial support for subject compensation and laboratory supplies.

Ethical Approval: This study was approved by the Institutional Review Board at Burrell College of Osteopathic Medicine, Las Cruces, NM (IRB\# 0079_2021). The study is also registered with ClinicalTrials.gov (NCT04926415).

Informed Consent: All study participants provided written informed consent.

Poster No.: ${ }^{\mathrm{B} 10}$

Abstract No.: 24

Category: Basic Science

Research Focus Area: Chronic Diseases \& Conditions

STK11 IP identification in double membrane vesicle composition of HCoV-0C43 infected cells by subcellular fractionation

${ }^{1}$ Jessica Lynn Brumbaugh, OMS-11; ${ }^{2}$ Eleana Estrella, OMS-11; ${ }^{2}$ Michelle Vanoy-Warner, BS; ${ }^{2}$ Crystal Boudreaux, PhD

${ }^{1}$ West Virginia School of Osteopathic Medicine (WVSOM); ${ }^{2}$ Department of Biomedical Sciences, West Virginia School of Osteopathic Medicine (WVSOM) ${ }^{3}$ Department of

Context: As the novel SARS-CoV-2 coronavirus spread across the globe, the race to find effective drug therapies began. However, a significant lack of understanding of the virus's ability exploit host cell mechanisms for viral replication and survival is still unknown. Following the osteopathic tenet of the body as a unit, this research investigates the dependence of HCoV-OC43 on host cell mechanisms for viral survival.

Objective: The objective of this research is to better understand the mechanism at which HCoV-OC43 exploits host mechanics by separating nuclear, mitochondrial, plasma membrane, and cytoplastic cellular fractions. In addition, the location of cellular proteins STK11IP, STK11, and mTOR were identified within these different fractions. Methods: MA104 cells (simian monkey kidney) were infected human CoV-CO43. Subcellular fractions of the nucleus, mitochondria, plasma membrane, and cytosol from cell samples were obtained using centrifugation. 
Samples were standardized by Coomassie stained gel electrophoresis. SDS-Page and transfers of the fractions where then performed and later used for viral and cellular protein probing with targeted antibodies. Antibody cellular markers were selected to confirm fractions from the Abcam database. Infection was established using COVID Spike and $7 \mathrm{a}$ in whole cell lysate. HCoV-OC43 infection was confirmed by cell culture cytoplastic effect (CPE).

Data analysis was done via western blot chemi image analysis for cellular and viral protein band expression. Known antibodies for target proteins were used to confirm the identity of subcellular fractions in MA104 infected and mock cells. Patterns of interest were scored based on whether proteins appeared at their expected molecular weight in expected or unexpected fractions.

Results: Selected antibodies were used to target host and viral proteins. Plasma membrane markers were found in infected cellular fractions of not only the predicted plasma membrane fraction but also nuclear and mitochondrial fractions. Plasma membrane markers were also found in uninfected samples of predicted nuclear fractions. Cytoplasm markers were found in both infected and uninfected nuclear, mitochondrial and plasma fractions but not in the infected or uninfected fractions the protocol predicted for cytoplasmic fractions. Early endoplasmic markers were found in infected mitochondrial and nuclear fractions and the uninfected nuclear fraction.

In addition, patterns of host and viral cellular protein (STK11IP, STK11, mTOR, COVID Spike and OR7a) location within fractions were analyzed to aid in understanding the molecular pathogenesis of the corona virus and how different components of the cells are affected during infection. STK11IP was identified within the uninfected cell sample for predicted nuclear fractions and infected mitochondrial and membrane fractions. This pattern is the same for antibodies confirming the location of plasma membranes. Markers for mTOR were found in the uninfected nuclear fractions and infected mitochondrial fractions. STK11 was only found in uninfected fractions predicted but not supported by antibody markers for cytoplasmic proteins.

Conclusion: Preliminary data suggest a unique correlation between the presence of plasma membrane antisodium potassium ATPase antibody marker in not only the infected fractions of plasma membrane but also the nuclear infected fractions and strongly in infected mitochondrial fractions. SARS-CoV replicates within the cytoplasm of infected cells through an elaborate but poorly understood double-membranes vesicles (DMVs) that form on the host endoplasmic reticulum (ER). Our results support the theory that SARS-CoV DMV replication complexes could be made of a mixture of modified host cell membranes including plasma and mitochondrial membranes. The DMVs are reported to be very fragile and difficult to preserve when ultrastructural studies are done explaining the presence of more antibody markers associated with the mitochondrial and nuclear fractions than the plasma membrane fraction. ${ }^{1}$

As infection progresses, DMVs become concentrated in the perinuclear area separated by mitochondria along the ER, this additionally supports a sperate theory of mitochondrial associated membranes (MAMs). Literature suggests that reorganization of host cell membranes to form DMVs is a defense mechanism of the virus to hide its sites of viral RNA synthesis. ${ }^{2}$ Future research can potentially use target proteins of the host reorganized membranes for theraputic measures to stop viral replication.

Based on our previous research, increased expression of STK11IP, an upstream regulator of STK11, modulates mTOR promoting viral replication by inhibition of the AMPK pathway. Our results show mirrored location markers for both plasma membrane and STK11-IP suggesting that STK11IP may be interacting with host plasma membranes for replication. The viral DMV theory of combined cellular membranes affirms the importance of an osteopathic approach even on a subcellular level.

\section{References}

1. Knoops K, Kikkert M, Worm SH, et al. SARS-coronavirus replication is supported by a reticulovesicular network of modified endoplasmic reticulum. PLoS Biol. 2008;6(9):e226. doi:10.1371/journal. pbio.0060226

2. Wolff G, Melia CE, Snijder EJ, Bárcena M. Double-Membrane Vesicles as Platforms for Viral Replication. Trends Microbiol. 2020;28(12):1022-1033. doi:10.1016/j.tim.2020.05.009

Financial Disclosures: None reported.

Support: West Virginia Clinical Translation Science Institute (WVCTSI) National Institutes of Health (NIGMS) 5U54GM10492-04

Ethical Approval: Exempt

Informed Consent: N/A 
Poster No.: *B11

Abstract No.: 25

Category: Basic Science

Research Focus Area: Chronic Diseases \& Conditions

Subcellular fractionation in rotavirus infected cells to evaluate the role of mitochondrial associated proteins

${ }^{1}$ Eleana Cristina Estrella, OMS-II; ${ }^{2} J e s s i c a$ Brumbaugh, OMS-II; ${ }^{2}$ Michelle Vanoy-Warner, BS; ${ }^{2}$ Crystal Boudreaux, $\mathrm{PhD}$

${ }^{1}$ West Virginia School of Osteopathic Medicine (WVSOM);

${ }^{2}$ Department of Biomedical Sciences, West Virginia School of Osteopathic Medicine (WVSOM)

Context: Rotavirus is the leading cause of diarrheal disease worldwide in children under five. ${ }^{1}$ Identifying the presence of viral proteins in subcellular fractions will help convey utilization of particular parts of host cell machinery to promote infection. An osteopathic approach aligns with the tenet that the body is a unit and by discerning the many ways the virus makes use of the different parts of the cell, one can piece together the regulation of viral replication as a whole within host cells.

Objective: The objective of this project is to determine which parts of the host cell rotavirus is exploiting for infection using subcellular fractionation of host cells. These fractions were identified by using targeted antibodies to confirm known proteins in the fraction. Host proteins such as STK11 (serine threonine kinase), STK11IP (serine threonine kinase 11 interacting protein), and mTOR (mechanistic target of Rapamycin) were also used to analyze the presence in different subcellular fractions.

Methods: MA104 cells (simian monkey kidney) were infected with simian rotavirus strain SA11 with and without Rapamycin treatment. Subcellular fractions of the nucleus, mitochondria, plasma membrane, and cytosol from SA11 samples were obtained using centrifugation. Samples were standardized by Coomassie stained gel electrophoresis. SDS-PAGE and transfers of the fractions where then performed and later used for viral and cellular protein probing with targeted antibodies. Antibody cellular markers were selected from the Abcam database. Rotavirus infection was confirmed using rotavirus non-structural protein NSP2.

Data analysis was done via western blot chemi image analysis for cellular and viral protein band expression. Known antibodies for target proteins were used to confirm the identity of subcellular fractions in MA104 infected and uninfected cells. Patterns of interest were scored based on whether proteins appeared at their expected molecular weights and in their expected fractions or if they appeared in other fractions.

Results: In order to visualize the proteins of interest, select antibodies were used in western blots. Plasma membrane markers were found not only in the membrane fraction but in the nuclear and mitochondrial fractions as well for infected and mock cells. Similar unexpected patterns occurred with the other protein antibodies (nuclear, cytosolic, early endosome) that were used for detection of subcellular fractions. In addition, patterns of host and viral cellular protein (STK11IP, STK11, mTOR, and NSP2) location within fractions were analyzed. STK11-IP markers were identified within the uninfected cell sample for nuclear, mitochondrial and membrane fractions all of which were confirmed with cell organelle markers. While mitochondrial fractions were the only location of STK11IP in infected cells. STK11 was also solely found in the mitochondrial fractions however, in both infected and uninfected cells. mTOR was found in mitochondrial mock fractions of uninfected cells.

Conclusion: Preliminary data suggests a correlation between the presence of plasma membrane antibody marker in both plasma membrane fractions of infected and noninfected fractions as well as the mitochondrial fractions of infected cells. This suggests that during infection the viroplasm is not only associated with the ER but with the mitochondria as well. This association may be through mitochondrial associated membranes (MAMs) which are subdomains of the ER that foster juxtaposition of the ER and mitochondria during accumulation of ER proteins. ${ }^{2}$ During infection the viroplasm attached to the ER may exploit the protein producing ability of the ER to make its own viral proteins and thus causing an increased production of ER proteins to ensure viral progeny can be made. MAMs are abundant in cholesterol and detectable in membranes of cellular organelles like the mitochondria and ER. The close approximation of the ER and mitochondria due to MAMs may help with membrane trafficking and cell signaling to further propagate infection of host cells. This preliminary data may add to the understanding of which host proteins the virus impacts during infection providing potential targets for therapy. Normalization of data with repeated subcellular fractions will help tailor the methods we use to get pure fractions without carry over. In addition, using more known antibodies would help identify cellular proteins found within each fraction. Future directions could include detection of a MAM marker present in both infected mitochondrial and ER membrane fractions. This would elucidate why 
membrane markers are being detected in fractions other than membranes themselves in infected cells. Clarifying this particular pathogenesis of the Rotavirus through organelle membranes could help with the understanding of the molecular hijacking used in other RNA viruses such as the Sars-CoV-2, which has significantly impacted the world and has become a pressing issue as it has taken the lives of millions.

\section{References}

1. Burnett E, Parashar U, Tate J. Rotavirus Vaccines: Effectiveness, Safety, and Future Directions. Paediatr Drugs. 2018;20(3):223233. doi:10.1007/s40272-018-0283-3

2. Garofalo T, Matarrese P, Manganelli V, et al. Evidence for the involvement of lipid rafts localized at the ER-mitochondria associated membranes in autophagosome formation. Autophagy. 2016;12(6):917-935. doi:10.1080/15548627.2016.1160971

Financial Disclosures: None reported.

Support: West Virginia Idea Network of Biomedical Research Excellence. NIH (NIGMS) Grant \#P20GM103434

Ethical Approval: Exempt

Informed Consent: None

Poster No.: ^B12

Abstract No.: 26

Category: Basic Science

Research Focus Area: Chronic Diseases \& Conditions

Investigating the Behavioral and Cortical Effects of Spinocerebellar Ataxia Type 11 Mice in Response to Electric Stimulation of the Cerebellar Cortex

\author{
${ }^{1}$ Madison Cohen, OMS-I; ${ }^{2} \mathrm{Huo} \mathrm{Lu}$ \\ ${ }^{1}$ Philadelphia College of Osteopathic Medicine-Georgia \\ Campus (PCOM-GA); ${ }^{2}$ Department of Biomedical Sci- \\ ences, Philadelphia College of Osteopathic Medicine- \\ Georgia Campus (PCOM-GA)
}

Context: The role of the cerebellum in coordination of movement is universally accepted, but the mechanisms underlying this role remain elusive. There is evidence to suggest that Purkinje cells (PC) have a role as the computational center of the cerebellar cortex ${ }^{1}$, however the link between PC function and animal behavior is not fully understood. The goal of this study is to observe the behavioral and neuronal changes associated with electric stimulation of the cerebellar cortex in SCA11 mice.

Objective: Hereditary spinocerebellar ataxia (SCA) affects people worldwide, occurring at an estimated global prevalence of 1 to 5 per 100,000 people. ${ }^{5}$ SCAs are a group of genetically inherited ataxias, caused by progressive degeneration of the cerebellum and associated regions of the nervous system. ${ }^{3}$ SCA11 mice expressing a malformed cerebellar Purkinje cell layer, show characteristic symptoms of cerebellar dysfunction during behavior that is similar to those observed in people affected by SCA11.

Methods: All animal procedures were in accordance with the protocol approved by the IACUC of PCOM-GA. Mice were purchased from JAX and housed in the LAR at PCOM Georgia. Mice were trained to walk on a self propelled wheel. Video recordings taken during experiments provided both lateral and inferior views of the animal. Videos of the experiment were captured using a camera and the Pylon Software. The onset of video recording was triggered externally, with the frame rate being between 80 and 100 fps. Video data was later analyzed using DeepLabCut (DLC), a markerless pose estimation program. Electric stimulation was applied to the cerebellar cortex using a concentric electrode with a tip size of 2 to $3 \mu \mathrm{m}$. Stimulation applied consisted of a $200 \mu \mathrm{A}$ pulse train of 105 pulses over a duration of $178.2 \mathrm{~ms}$. An FHC microelectrode with a resistance of $1 \mathrm{M} \Omega$ was used to collect LFP recordings from the contralateral primary motor cortex. LFP signals were collected using pCLAMP at a sampling frequency of $10 \mathrm{kHz}$ using a band filter from $1 \mathrm{~Hz}$ to $20 \mathrm{kHz}$. Since PCs are the sole output of the cerebellar cortex and it is known that these cells are dysfunctional in $\mathrm{SCA}^{2}$, a computational neuronal model of a cerebellar Purkinje cell was used to simulate the effects of alterations of Purkinje cell morphology and synaptic properties affect cell function. In order to analyze locomotion of mice while on the wheel an open-source markerless pose estimation program, called DLC was used. ${ }^{4}$ MATLAB was then used for further analysis of DLC data and plotting. Gait parameters observed and analyzed using matlab included stride length, stride width, limb velocity, and step cycle. LFP recordings were plotted against time in order to observe the summation of activity in the primary motor cortex. Furthermore, a power spectrum was used to display the dominant frequencies present both before and after stimulation. The correlation between stepping frequency and dominant LFP frequency was studied.

Results: The key finding of this study was that there was a significant difference between control and ataxic mice in both local field potential and corresponding behavior. Furthermore, the frequency of local field potential activity 
correlated with the frequency of behavior for both control and ataxic mice. Electric stimulation of the cerebellar cortex did not cause significant changes in behavior or local field potential activity for both control and ataxic mice. A computational model of a cerebellar Purkinje cell showed that decreasing the dendritic arborization of the cell as well as removing metabotropic glutamate receptor type 1 caused the cell to decrease in firing rate.

Conclusion: In this study a significant difference between the behavior of control and SCA11 mutant mice was apparent. Upon analysis of the gait of control and ataxic mice it was found that ataxic mice displayed a significantly shorter stride length, lower frequency of stepping, and a slower limb velocity as compared to that of control mice. These results are consistent with the expected ataxic phenotype. Furthermore, LFP activity was strongly correlated with frequency of stepping for both control and ataxic mice. The key finding of this study is that there is a significant difference between control and ataxic mice in LFP activity and the associated behavior. This finding highlights an association between cerebellar dysfunction and changes in motor cortical LFP activity that correlate with an ataxic phenotype. Computational modeling of a cerebellar Purkinje cell provided a better way for us to understand the mechanism behind the morphological and synaptic changes associated with SCA. It was found that decreasing the dendritic arborization of the cell as well as removing mGluR1 caused the cell to decrease in firing rate. These findings suggest that cerebellar Purkinje cell deformations lead to a decrease in action potential generation and subsequent alteration of cerebellar output that results in abnormal motor cortical activity and behavior that is seen in SCA.

\section{References}

1. Albus, J. S. (1971). A theory of cerebellar function. Mathematical biosciences (pp. 25-61). Greenbelt, Maryland: American Elsevier Publishing Company.

2. Hoxha, E., Balbo, I., Miniaci, M. C., \& Tempia, F. (2018). Purkinje cell signaling deficits in animal models of ataxia. Frontiers in Synaptic Neuroscience, 10, 6. doi:10.3389/fnsyn.2018.00006 [doi]

3. Klockgether, T., Mariotti, C., \& Paulson, H. L. (2019). Spinocerebellar ataxia. Nature Reviews Disease Primers, 5(1), 24. doi:10. 1038/s41572-019-0074-3

4. Mathis, A., Mamidanna, P., Cury, K. M., Abe, T., Murthy, V. N., Mathis, M. W., \& Bethge, M. (2018). DeepLabCut: Markerless pose estimation of user-defined body parts with deep learning. Nature Neuroscience, 21(9), 1281-1289. doi:10.1038/s41593018-0209-y
5. Ruano L, Melo C, Silva MC, Coutinho P. The global epidemiology of hereditary ataxia and spastic paraplegia: a systematic review of prevalence studies. Neuroepidemiology. 2014;42(3):174-83. doi: 10.1159/000358801. Epub 2014 Mar 5. PMID: 24603320.

Financial Disclosures: None reported.

Support: None reported.

Ethical Approval: All animal procedures were in accordance with the protocol approved by the IACUC (IACUC number: \#A19-004) of PCOM-GA.

Informed Consent: N/A

Poster No.: ${ }^{\star} \mathrm{B} 13$

Abstract No.: 27

Category: Basic Science

Research Focus Area: Chronic Diseases \& Conditions

The differential roles of AMPK $y$ subunit isoforms on doxorubicininduced $\mathrm{H} 9 \mathrm{C} 2$ cell death

${ }^{1}$ Vignesh Gunasekaran, OMS-II; ${ }^{2}$ Naunihal Singh, OMS-II; ${ }^{2}$ Qiangrong Liang, MD, PhD; ${ }^{2}$ Satoru Kobayashi, PhD; ${ }^{2}$ Tamayo Kobayashi, MS

${ }^{1}$ New York Institute of Technology (NYITCOM); ${ }^{2}$ Department of Biomedical Sciences, New York Institute of Technology (NYITCOM)

Context: The usage of the anticancer drug Doxorubicin (DOX) is limited due to the severe cardiotoxicity through poorly defined mechanisms. AMP-activated protein kinase (AMPK) is an essential cellular energy homeostasis regulator which supports cell survival. AMPK activation is speculated to be protective against DOX-induced cardiomyopathy. However, there has been limited investigation about the role of the different subunits of AMPK or their individual isoforms in DOX-induced cardiomyocyte injury.

Objective: To investigate the effects of knocking down isoforms of the energy-sensing $y$ subunit of AMPK on DOX-induced cytotoxicity in cardiac cells.

Methods: H9c2 cardiomyoblast cells were treated with siRNA to knock down the expression of $y 1, y 2$, or both and then exposed to DOX. Cell death was determined by propidium iodide (PI) staining that indicates necrosis and Western blot analysis of the expression levels of cleaved caspase 3 that signals apoptosis.

Results: Our findings show that knockdown of the $y 1$, but not the $y 2$, isoform induces increased H9c2 necrosis compared to the control treatment, as shown by an increase in PI positive cells, without increasing cleaved 
caspase 3 expression. In the DOX-treated H9c2 cells, there is no further cell death induced by $y 1$ knockdown. Interestingly however, while DOX treatment induced apoptosis in the control cells, shown by increased expression of cleaved caspase 3 compared to non-DOX treated control cells, the increase in cleaved caspase 3 expression was reversed to normal levels in the $y 1$ knockdown cells. The effects of $y 2$ knockdown were minimal either in the $y 2$ alone or both $y 1$ and $y 2$ siRNA-treated cells.

Conclusion: These findings suggest that AMPK $y 1$ isoform may play a role to induce apoptosis but not necrosis in response to DOX.

References N/A

Financial Disclosures: This study is supported by NIH grant 1R15HL137130-01A1.

Support: None reported.

Ethical Approval: N/A

Informed Consent: N/A

Poster No.: *B14

Abstract No.: 28

Category: Basic Science

Research Focus Area: Chronic Diseases \& Conditions

P21-Activated Kinase 1 is Essential for the Gene Expression of the Autophagy Adaptor P62/SQSTM1 in Cardiomyocytes

${ }^{1}$ Shawn Geffken, OMS-II; ${ }^{2}$ Tint Tha Ra Wun; ${ }^{2}$ Tamayo Kobayashi; ${ }^{2}$ Satoru Kobayashi, PhD; ${ }^{2}$ Qiangrong Liang, $\mathrm{MD}, \mathrm{PhD}$

${ }^{1}$ New York Institute of Technology (NYITCOM); ${ }^{2}$ Department of Biomedical Sciences, New York Institute of Technology (NYITCOM)

Context: P21-Activated Kinase 1 (PAK1) is a serinethreonine kinase that plays a protective role in cardiomyocytes under several stressful conditions through unknown mechanisms. We have previously demonstrated that PAK1 is essential for maintaining autophagy and mitophagy activities. However, the downstream mediator remains to be identified. P62 (Sequestosome 1/SQSTM1) is a multifunctional adaptor protein involved in numerous cellular functions including autophagy and mitophagy.

Objective: In this study, we investigated the relationship between PAK1 and p62 in the regulation of autophagy and mitophagy in $\mathrm{H} 9 \mathrm{c} 2$ cardiac myoblast cells.

Methods: PAK1 was knocked down in H9c2 cardiac myoblast cells by using siRNA and the expression level of p62 was determined by Western blot analysis. Meanwhile, the autophagy flux and mitophagy flux were determined by measuring LC3-II protein levels in total cell lysates and mitochondrial fractions with and without the lysosomal inhibitors pepstatin A (pepA) and E64d, respectively. The qualitative imaging results for the expression of p62, the autophagy flux, and the mitophagy flux obtained from Western blot detection were quantified using Fiji ImageJ software. The collected numerical data were analyzed and presented in graphical format using Prism software. One of the tenets of osteopathy emphasizes the body's ability to self-regulate, heal, and maintain homeostasis. Understanding the role of p62 in the PAK1 autophagy and mitophagy signaling pathways is crucial in establishing therapies that restore cellular homeostasis and in turn improve outcomes in patients with various heart diseases.

Results: The results showed that PAK1 knockdown substantially reduced p62 protein levels in the total cell lysates. PAK1 knockdown also decreased LC3-II levels in the total cell lysates, which were not significantly increased by the lysosomal protease inhibitors pepstatin A (pepA) and E64d, suggesting that autophagy flux is reduced by downregulation of PAK1. Similarly, PAK1 knockdown concurrently reduced the expression levels of p62 and LC3-II in the mitochondria fractions either in the presence or absence of pepA and E64d, indicating limited mitochondrial degradation or mitophagy in the absence of PAK1.

Conclusion: Together, our results demonstrated a clear correlation between decreased p62 levels and reduced autophagy flux and mitophagy flux upon PAK1 downregulation, raising the possibility that $\mathrm{p} 62$ may be a downstream mediator that is responsible for PAK-dependent autophagy and mitophagy. Further studies are warranted to determine whether restoring p62 levels can overcome the inhibition of autophagy and mitophagy by PAK1 deficiency. It is equally important to elucidate how PAK1 knockdown can lead to the reduced p62 expression.

\section{References}

1. Kichina JV, Goc A, Al-Husein B, Somanath PR, Kandel ES. PAK1 as a therapeutic target. Expert Opin Ther Targets. 2010;14(7):703-725. doi:10.1517/14728222.2010.492779

2. Lippai M, Lőw P. The role of the selective adaptor p62 and ubiquitinlike proteins in autophagy. Biomed Res Int. 2014;2014:832704. doi:10.1155/2014/832704

3. Bao J, Li G, Yuan X, Li PL, Gulbins E. Contribution of p62 to Phenotype Transition of Coronary Arterial Myocytes with Defective 
Autophagy. Cell Physiol Biochem. 2017;41(2):555-568. doi:10. $1159 / 000457877$

Financial Disclosures: None reported.

Support: NIH grant 1R15HL137130-01A1

Ethical Approval: Exempt

Informed Consent: Not Relevant.

${ }^{\star}$ Poster No.: ${ }^{\star B} 15$

Abstract No.: 29

Category: Basic Science

Research Focus Area: Chronic Diseases \& Conditions

Characterizing Calciphylaxis-like Lesions in Mice Predisposed to Vascular Calcification

${ }^{1}$ Lesley Santos, OMS-II; ${ }^{2}$ Lara Tong, OMS-II; ${ }^{2}$ Olga Savinova, $\mathrm{PhD}$

${ }^{1}$ New York Institute of Technology (NYITCOM); ${ }^{2}$ Department of Biomedical Sciences, New York Institute of Technology (NYITCOM)

Context: Tissue nonspecific alkaline phosphatase (TNAP) can cause osteoblastic transformation of vascular cells, contributing to vascular hydroxyapatite crystallization. ${ }^{1,2}$ Calciphylaxis is a rare yet fatal form of vascular calcification often associated with end-stage renal disease (uremic calciphylaxis), but also with non-uremic causes. ${ }^{3}$ Thrombotic occlusion leads to chronic skin ulcers and necrotic lesions prone to superinfection. Risk factors include obesity, diabetes, and warfarin use.

Objective: Our study aims to validate a mouse model of non-uremic calciphylaxis secondary to alkaline phosphatase overexpression in the vasculature. Histological and micro-CT evaluation of trunk skin samples will help determine if the lesions are characteristic of calciphylaxis. We hypothesized that TNAP upregulation would lead to medial calcification and intima fibrosis of small arterioles. Consequential thrombosis and tissue hypoxia will result in skin lesions characteristic of early-stage calciphylaxis.

Methods: Our experiment used tissues from a previous study, archived in formalin. Skin was sampled from the trunks of 9 mice with TNAP-induced vascular calcification and 9 wild type (WT) mice that served as the controls. Trunk regions were chosen as they are a common location for human calciphylaxis lesions. ${ }^{3}$ Tissue samples were prepared as cryosections on TruBond ${ }^{\mathrm{TM}}$ adhesive slides to increase tissue adherence. Tears and holes were often formed when creating cryosections of calcified tissues. Thus, sections with the best preservation of tissue morphology were collected. Calcium deposits and morphological changes were characterized histologically using Alizarin and H\&E (hematoxylin and eosin) stains, respectively. Modifications were made to the Alizarin protocol to improve tissue adhesion. Slides were laid on a flat surface, and a micropipette was used to flood the tissues with $200 \mu \mathrm{L}$ of $20 \mathrm{mM}$ Alizarin Red. Afterward, a transfer pipette was used to rinse the tissues with PBS three times.

Tissues from 6 TNAP mice and 5 WT mice were also dissected and observed via micro-CT to confirm the presence of calcium. The amount of calcification was also measured volumetrically from micro CT reconstruction. From a related cohort, 1 TNAP mouse and 1 WT mouse were perfused with a vascular contrast resin (Microfil ${ }^{\circledR}$ MV-122 Yellow, Flow Tech, Inc.). Trunk tissues from these mice were then dissected, and arterial vasculature was reconstructed via micro-CT. Data were analyzed using a twotailed student's t-test using GraphPad Prism statistical software. Significance was accepted at $\mathrm{p}<0.05$.

A holistic approach to patient care makes osteopathic physicians uniquely equipped to manage ischemia patients. Osteopathic manipulative treatments (OMT) such as myofascial thoracic outlet release, diaphragm doming, pelvic diaphragm release, and pedal pump may improve lymphatic flow and restore blood flow. Evidence suggests OMT reduces edema and subsequent healing time for venous ulcers. ${ }^{4}$

Results: Both Alizarin and H\&E confirmed the presence of calcium deposits in the skin of mice with TNAP upregulation. These mice, as well as the WT mice, were all 23-week-old males. The calcification appeared to be primarily vascular in nature and localized to the dermis and subcutaneous adipose tissue. Incidentally, we also found that the TNAP mice lost the typical morphology of skin structures (such as hair follicles) compared to WT mice tissues.

Micro-CT analysis of non-perfused and perfused mice tissues further confirmed the presence as well as the extent of calcification. Non-perfusion micro-CT revealed that calcification was present in 100\% of TNAP mice compared to the complete absence of vascular calcification in WT mice. The calcification in the affected mice appeared to follow a vascular pattern and to be localized to the dermis and subcutaneous adipose tissue. Perfusion micro-CT confirmed both the vascular nature and the location of the calcification. Perfusion micro-CT also revealed that tissues of the TNAP mice had been incompletely perfused, meaning that the contrast resin did not fill the full diameter of each blood vessel. 
Conclusion: Our histological findings support the hypothesis that TNAP upregulation leads to a calciphylaxislike condition of the skin. The presence of vascular calcification in adipose-rich trunks of the TNAP mice is consistent with the pattern of lesion formation in human calciphylaxis patients. Incomplete perfusion of TNAP mice tissues, as observed via micro CT, may suggest that calcified deposits occluded the blood vessels.

Observations from our mice tissues are consistent with early-stage human calciphylaxis. According to a previously described histological and cutaneous schema, our mice tissues are most reflective earliest histologic manifestations. 5 These early stages lack ulcerations or necrotic lesions, however, they may be characterized by gross skin changes with intact epithelium. The fur coat on our mice made it difficult to identify signs of purpura, livedo reticularis, or other skin changes of this nature that may be present in early-stage human calciphylaxis. ${ }^{2,5}$

Our study had a few limitations. The mice we used did not have gross skin lesions, as is typical of human calciphylaxis at the time of diagnosis. Thus, all of our observations were done purely microscopically. Additionally, some of the TNAP mice sections were more difficult to analyze histologically due to the sections tearing at calcification sites.

While there is limited literature on uremic calciphylaxis, its non-uremic counterpart is even less understood. Detection of calciphylaxis-like lesions in TNAP mice provided supporting evidence for the TNAP activity as a target gene for non-uremic calciphylaxis. Future studies comparing the histological findings to human lesions with TNAP upregulation and the curative potential of TNAP knockout mutations for calciphylaxis may be of interest. Advances in this research may allow dermatologists and primary care physicians to recognize calciphylaxis and begin proper management leading to a better prognosis.

\section{References}

1. Sheen CR, Kuss P, Narisawa S, Yadav MC, Nigro J, Wang W, Chhea TN, Sergienko EA, Kapoor K, Jackson MR, Hoylaerts MF, Pinkerton AB, O'Neill WC and Millan JL. Pathophysiological role of vascular smooth muscle alkaline phosphatase in medial artery calcification. J Bone Miner Res. 2015;30:824-36.

2. Savinov AY, Salehi M, Yadav MC, Radichev I, Millan JL and Savinova OV. Transgenic Overexpression of Tissue-Nonspecific Alkaline Phosphatase (TNAP) in Vascular Endothelium Results in Generalized Arterial Calcification. Journal of the American Heart Association. 2015;4.
3. Jeong HS and Dominguez AR. Calciphylaxis: Controversies in Pathogenesis, Diagnosis and Treatment. Am J Med Sci. 2016;351:217-27.

4. Kilgore T, Malia M, Di Giacinto B, Minter S and Samies J. Adjuvant Lymphatic Osteopathic Manipulative Treatment in Patients With Lower-Extremity Ulcers: Effects on Wound Healing and Edema. J Am Osteopath Assoc. 2018;118:798-805.

5. Dutta P, Chaudet KM, Nazarian RM, Kroshinsky D and Nigwekar SU. Correlation between clinical and pathological features of cutaneous calciphylaxis. PloS one. 2019;14:e0218155.

Financial Disclosures: None reported.

Support: The acquisition of the microCT scanner was made possible by a Major Research Instrumentation (MRI) grant from the National Science Foundation (NSF 1828305) to PIs Simone Hoffmann, Julia Molnar, Azhar Ilyas, Olga Savinova, and Claude Gagna and with the support of NYIT's College of Osteopathic Medicine (NYITCOM), College of Engineering and Computing Sciences, and College of Arts and Sciences. LS received summer research support for NYITCOM. The funders were not involved in study design or execution.

Ethical Approval: Animal studies were approved by the New York Institute of Technology College of Osteopathic Medicine (Old Westbury, NY) and complied with the National Institutes of Health guidelines for humane treatment of laboratory animals.

Informed Consent: N/A

Poster No.: *B16

Abstract No.: 31

Category: Basic Science

Research Focus Area: Chronic Diseases \& Conditions

Downregulated Endothelial TissueNonspecific Alkaline Phosphatase Does Not Affect Calcification but Reduces Cardiac Function in Atherosclerotic Mice

${ }^{1}$ Sandy W. Than, OMS-II; ${ }^{2}$ Maria Canellos, OMS-II; ${ }^{2}$ Ethan Shamsian, OMS-II; ${ }^{2}$ Nimmy Joseph; ${ }^{2}$ Mohnish Singh, MS; ${ }^{2}$ Olga V. Savinova, $\mathrm{PhD}$

${ }^{1}$ New York Institute of Technology (NYITCOM); ${ }^{2}$ Department of Biomedical Sciences, New York Institute of Technology (NYITCOM)

Context: No therapy is available currently to reverse vascular calcification.1 We have previously shown that 
endothelial cells can promote vascular calcification and overexpression of tissue-nonspecific alkaline phosphatase (TNAP), a regulating enzyme in biomineralization, can increase osteogenic potential of endothelial cells and accelerate atherosclerosis. 2,3 However, whether TNAP activity in vascular endothelium is essential for intimal calcification and the progression of atherosclerosis is unknown.

Objective: To determine whether elimination of TNAP expression in endothelial cells would be sufficient to reduce intimal calcification associated with atherosclerosis.

In line with osteopathic principal of interconnectedness between structure and function, we sought out to see how the body attempts to keep up normal cardiac function in the presence of altered gene expression, how much it takes to induce physiological changes with this allostatic load, and if the gene expression changes these odds.

Methods: To produce endothelial-specific WHC-eTNAP knockout (KO) mice, a mouse model was introduced with human alkaline phosphatase gene (alpl), which encodes TNAP, and intercrossed with endothelial-specific CRE recombinase mice. CRE was expressed under the control of the Tie2 gene promoter. To create hypercholesterolemic conditions, a mouse model with a homozygous recessive wicked high cholesterol (WHC) mutation in the low density lipoprotein receptor (LDLR) was used. Endothelial TNAP KO $(n=8)$ and control $(n=9)$ mice were fed an atherogenic Western diet starting from 8 weeks of age and until 52 weeks of age.

At 52 weeks of age, echocardiography was performed using a Vevo3100 imaging system (Fujifilm Visual Sonics). Mice were anesthetized with $1 \%$ - $2 \%$ isoflurane, and parasternal short axis view was obtained in B-mode and recorded in M-mode. Heart rate was maintained at greater than $400 \mathrm{bpm}$, and the anesthesia level was adjusted as needed. M-mode echocardiograms were analyzed by tracing myocardial wall movement over 3 to 5 cardiac cycles with VevoLab imaging analysis software to measure cardiac time interval, left ventricular (LV) diameter and LV wall thickness in systole and diastole, and to calculate heart rate, LV mass, ejection fraction, cardiac output, and stroke volume.

Calcium volume in the aortic root and aortic arch area was obtained ex vivo with microcomputed tomography (microCT) instrument SkyScan 1173 (MicroPhotonics). 3D image stacks were reconstructed from the rotation image projections using NRecon software (MicroPhotonics). Reconstructed images were analyzed and quantified using Dragonfly 4.1 program (Object Research Systems Inc).

Data were expressed as mean \pm SD. GraphPad Prism version 7 (GraphPad Software) was used for all analyses.
Data were analyzed using a 2-way ANOVA to calculate the effects of the genotype adjusted for sex. The significance was accepted at $\mathrm{p}<0.05$.

Results: MicroCT data did not show a statistically significant decrease in calcification in the aortic root or arch area in endothelial TNAP KO mice compared to controls; however, several cardiac parameters were affected by genotype. The physiological examination of the mice demonstrated that ablation of TNAP expression in the endothelium resulted in a significant reduction in ejection fraction $(29 \%$ vs. $46 \%$, $\mathrm{p}<0.05$ ), stroke volume (0.94 vs. $1.51 \mathrm{ul} / \mathrm{g}, \mathrm{p}<0.05)$, and cardiac output ( $\left.0.43 \mathrm{vs.} 0.70 \mathrm{ml} /\left(\mathrm{min}^{\star} \mathrm{g}\right), \mathrm{p}<0.05\right)$ in the absence of cardiac hypertrophy.

Conclusion: Although overexpression of TNAP has previously been implicated in increasing vascular calcification and atherosclerosis, our observation of TNAP downregulation did not show any effect on natural progression of calcified atherosclerosis. Our data, however, indicate that TNAP is required for the protection of cardiac function under conditions of atherosclerosis in mice. The optimal expression of TNAP where it offers cardioprotective properties without an increase in vascular calcification warrants further investigations.

\section{References}

1. Sage AP, Tintut Y, Demer LL. Regulatory Mechanisms in Atherosclerotic Calcification. Nat Rev Cardiol. 2010;7(9):528-536. doi:10. 1038/nrcardio.2010.115

2. Savinov AY, Salehi M, Yadav MC, Radichev I, Millán JL, Savinova OV. Transgenic Overexpression of Tissue-Nonspecific Alkaline Phosphatase (TNAP) in Vascular Endothelium Results in Generalized Arterial Calcification. J Am Heart Assoc. 2015;4(12). doi:10. 1161/JAHA.115.002499

3. Romanelli $F$, Corbo A, Salehi $M$, et al. Overexpression of tissuenonspecific alkaline phosphatase (TNAP) in endothelial cells accelerates coronary artery disease in a mouse model of familial hypercholesterolemia. Aikawa E, ed. PLOS ONE. 2017;12(10):e0186426. doi:10.1371/journal.pone.0186426

Financial Disclosures: None reported.

Support: The acquisition of the microCT scanner was made possible by a Major Research Instrumentation (MRI) grant from the National Science Foundation (NSF 1828305) to PIs Simone Hoffmann, Julia Molnar, Azhar Ilyas, Olga Savinova, and Claude Gagna and with the support of NYIT's College of Osteopathic Medicine (NYITCOM), College of Engineering and Computing Sciences, and College of Arts and Sciences. Ethical Approval: Animal studies were approved by the New York Institute of Technology College of Osteopathic Medicine (Old Westbury, NY) and complied with the 
National Institutes of Health guidelines for humane treatment of laboratory animals.

Informed Consent: N/A

Poster No.: *B18

Abstract No.: 38

Category: Basic Science

Research Focus Area: Chronic Diseases \& Conditions

Measuring Circulating Thyroid Hormones in Mice with TissueNonspecific Alkaline Phosphatase Induced Heart Failure

\author{
${ }^{1}$ Nancy Kallimanis, OMS-II; ${ }^{2}$ Olga V. Savinova, PhD \\ ${ }^{1}$ New York Institute of Technology (NYITCOM); ${ }^{2}$ Depart- \\ ment of Biomedical Sciences, New York Institute of Tech- \\ nology (NYITCOM)
}

Context: A relationship exists between cardiac tissue thyroid hormone (TH) levels and dilated heart failure (HF). THs contribute to contractile and relaxation forces in the heart; decreased THs are associated with a worse prognosis in HF patients. ${ }^{1}$ Treatment with THs benefits HF patients. ${ }^{2}$ Overexpression of tissue-nonspecific alkaline phosphatase (TNAP) in the vascular endothelium induces vascular calcification manifesting as coronary artery disease (CAD) and HF in a mouse model (TNAP transgenic mice). ${ }^{3}$

Objective: To determine if mice with TNAP induced HF show decreased levels of circulating TH in the blood. It is hypothesized that there will be decreased levels of circulating TH in TNAP HF mice when compared to the wild type (WT) mice. The osteopathic principle of unity of the body is used by investigating the relationship between THs and cardiac outcomes.

Methods: In this study, plasma samples from TNAP mice on the C57BL/6 genetic background were compared to control WT mice. We noted that mice on the pure $\mathrm{C} 57 \mathrm{BL} / 6$ genetic background were more susceptible to $\mathrm{CAD}$ and $\mathrm{HF}$ compared with the original TNAP stain on mixed genetic background. HF in the TNAP mice was defined as reduced ejection fraction shown on an echocardiogram. The circulating THs consist of the active hormone Triiodothyronine (T3) and precursor hormone Thyroxine (T4). T3 can either be measured bound to transport proteins or unbound as free T3. AccuBind Enzyme-linked immunosorbent assay (ELISA) microwells were used to detect plasma levels of T3, Free T3, and T4, which were used according to the manufacturer's recommended protocol. Our sample consisted of 17 WT and 12 TNAP mice. T3 serum reference ranges included 7.5, 5.0, 2.5, 1.0, 0.5, $0.25,0.125$, and $0 \mathrm{ng} / \mathrm{dl} .50 \mathrm{mul}$ of serum reference, WT plasma sample, or TNAP plasma sample were pipetted in duplicate. Free T3 serum reference ranges included 19, 8.6, 5.4, 3.1, 1.4, 0.7, and $0.75 \mathrm{pg} / \mathrm{ml} .50 \mathrm{mul}$ of serum reference, WT plasma sample, or TNAP plasma sample were pipetted in duplicate. T4 reference ranges included 25.0, 15.0, 10.0, 5.0, 2.0, 1.0, and 0.5 mug/dl. $20 \mathrm{mul}$ of serum reference, WT plasma sample, or TNAP plasma sample were pipetted in duplicate. Statistical analysis was performed using IBM SPSS Statistics to compare thyroid hormone levels in the TNAP group to the WT group.

Results: The mean ejection fraction percentage difference between the TNAP group $(29.6 \pm 18.4)$ and the WT group $(57.3 \pm 15.9)$ was statistically significant $(\mathrm{p}=0.004)$

Unpaired t-tests were performed to compare the mean levels of T3, Free T3, and T4 in WT and TNAP mice. T3 showed no statistical difference in the means of the TNAP group $(1.310 \pm 0.720)$ vs. the WT group $(1.330 \pm 0.261$, $\mathrm{p}=0.916$ ). Free T3 showed no statistical difference in the means of the TNAP group $(3.403 \pm 1.035)$ vs. the WT group $(3.765 \pm 0.537, p=0.229)$. T4 showed no statistical difference in the means of the TNAP group $(5.476 \pm 2.641)$ vs. the WT group ( $4.824 \pm 2.950, p=0.546)$.

Conclusion: The results of this study show no relationship between HF and change in levels of circulating THs in a mouse model. This suggests that ischemic HF in a mouse model may develop prior to the onset of detectable imbalances in circulating TH levels. Some limitations that may be responsible for this include a faster onset of HF in the TNAP mice ( 8 weeks) relative to the human disease and the lack of cardiac tissue T3 measurements. Other limitations of this study include relatively small sample size and limited availability of plasma samples which lead to not all samples being measured as duplicates. Current research suggests that serum thyroid levels are not always a reliable indicator of tissue thyroid levels and may underestimate cardiac tissue levels2. We suggest future studies measure cardiac tissue fetal gene levels, which can act as a measurement of tissue T3 levels. We would also suggest measuring serum levels of thyroidstimulating hormone (TSH), which indicate subclinical levels of hypothyroidism when elevated with a normal free T3 level. 


\section{References}

1. Gerdes AM, \& lervasi G. Thyroid replacement therapy and heart failure. Circulation. 2010;122(4):385-393. doi:10.1161/circulationaha. 109.917922

2. Gerdes, AM, \& Ojamaa K. Thyroid Hormone and Cardioprotection. Comprehensive Physiology 2016;6(3): 1199-1219. doi:10.1002/ cphy.c150012

3. Savinov AY, Salehi M, Yadav MC, Radichev I, Millán JL, \& Savinova OV. Transgenic Overexpression of Tissue-Nonspecific Alkaline Phosphatase (TNAP) in Vascular Endothelium Results in Generalized Arterial Calcification. Journal of the American Heart Association 2015;4(12). doi:10.1161/jaha.115.002499

Financial Disclosures: None reported.

Support: None reported.

Ethical Approval: Animal studies were approved by the New York Institute of Technology IACUC; protocol number 2019-OS-01

Informed Consent: N/A

Poster No.: *B19

Abstract No.: 39

Category: Basic Science

Research Focus Area: Chronic Diseases \& Conditions

Ablation of Tissue-Nonspecific
Alkaline Phosphatase In Macro-
phages Does Not Affect Athero-
sclerotic Plaque Calcification Or
Cardiovascular Physiology

${ }^{1}$ Ethan Shamsian, OMS-II; ${ }^{2}$ Nimmy Joseph, BS; ${ }^{2}$ Maria Canellos, BS; ${ }^{2}$ Sandy Than, BS; ${ }^{3}$ Mohnish Singh, MS; ${ }^{3}$ Olga Savinova, PHD

${ }^{1}$ New York Institute of Technology (NYITCOM);

${ }^{2}$ Medical Student, New York Institute of Technology (NYITCOM);

${ }^{3}$ Department of Research, New York Institute of Technology (NYITCOM)

Context: In patients who develop atherosclerosis, inflammation upregulates tissue-nonspecific alkaline phosphatase (TNAP) activity in vessel walls that overlaps with early signs of calcium deposition in the atherosclerotic plaque. ${ }^{1}$ Our preliminary study showed that increased expression of TNAP in macrophages exacerbates atherosclerotic calcification in a mouse model. However, whether or not TNAP is necessary in the progression of calcification, or for proper cardiac function, has yet to be studied.
Objective: The purpose of this study was to determine the effect of TNAP ablation in macrophages on atherosclerotic plaque calcification and cardiovascular physiology, and compare it to a control situation.

Methods: Macrophage-specific WHC-mTNAP knockout (KO) mice were produced in our colony by intercrossing floxed alpl transgenic mice (alpl gene encodes TNAP) and macrophage-specific CRE recombinase mice, in which CRE was expressed under the control of the lysozyme gene promoter. The macrophage TNAP KO strain was developed on the background of homozygous WHC ("wicked high cholesterol") mutation in the low density lipoprotein receptor gene. WHC-TNAP KO $(n=10)$ and their WHC littermates $(n=9)$ were placed on an atherosclerosis inducing diet at 8 weeks of age. The mice were maintained on the diet for 44 weeks, or until they turned 52 weeks. Microcomputed tomography (microCT) analyses of calcification in the aortic roots and arches were performed ex vivo. Echocardiographic studies were performed to assess cardiac structure and function at 52 weeks of age. Data were analyzed via a 2-way ANOVA to calculate the effect of TNAP ablation on calcification and cardiovascular physiology while adjusting for sex.

While the efficacy and application of OMM are limited in animal models, these models can inform us about the function of the body as a unit in health and disease. ${ }^{2}$ As illustrated by this work, a biomedical hypothesis can be framed to understand the interaction between more than one organ system. Here we investigated whether a specific genetic deficiency in myeloid cells of the immune system can affect the course of vascular pathology and cardiac function. Moreover, the study was conducted on a background of a genetic mutation in the low-density lipoprotein (LDL) receptor, which is expressed in the liver and is essential for the elimination of LDL cholesterol from circulation. Collectively, our approach to basic science research follows the fourth tenet of Osteopathic Medicine, stating that - "Rational treatment is based upon an understanding of the basic principles of body unity, self-regulation, and the interrelationship of structure and function."

Results: CT data showed no significant difference in calcification levels between the two genotypes $(\mathrm{p}=0.4702)$ of the mice. The WHC control mice and WHC-TNAP KO mice had a mean calcification level of $0.1456 \mathrm{~mm}^{\wedge} 3$ and $0.1004 \mathrm{~mm}^{\wedge} 3$, respectively (3.292\% difference). Cardiac parameters such as left ventricular (LV) mass/body weight (BW) $(p=0.2248)$, ejection fraction $(E F)(p=0.2136)$, and cardiac output (CO)/BW ( $\mathrm{p}=0.0843$ ) also showed no significant difference between the two genotypes. The WHC control mice and WHC-TNAP KO mice had a mean LV mass/ 
BW of $6.287 \mathrm{mg} / \mathrm{g}$ and $5.427 \mathrm{mg} / \mathrm{g}$, respectively $(9.385 \%$ difference). The WHC control mice and WHC-TNAP KO mice had a mean EF of $46.64 \%$ and $35.05 \%$, respectively $(6.878 \%$ difference). The WHC control mice and WHC-TNAP KO mice had a mean CO/BW of $0.7141 \mathrm{ml} /\left(\mathrm{min}^{\star} \mathrm{g}\right)$ and $0.4921 \mathrm{ml} /$ $\left(\mathrm{min}^{\star} \mathrm{g}\right.$ ), respectively (13.87\% difference).

Conclusion: Our data suggest that, despite TNAP expression in macrophages being sufficient to induce calcification of atherosclerotic plaques, it is not necessary for the calcification process. To the extent of our study, the ablation of TNAP in macrophages does not appear to have any consequences in a mouse model of atherosclerosis. Future studies should focus on TNAP ablation in another organ that produces endogenous TNAP such as the liver. ${ }^{3}$ Additionally, other methods such as detecting osteocalcin with immunohistochemistry can be utilized to examine calcium levels ${ }^{4}$ Histology of the heart tissues could also be analyzed with alizarin red to locate calcium deposits. ${ }^{5}$

\section{References}

1. Aikawa E, Nahrendorf M, Figueiredo J-L, et al. Osteogenesis Associates With Inflammation in Early-Stage Atherosclerosis Evaluated by Molecular Imaging In Vivo. Circulation. 2007;116(24):2841-2850. doi:10.1161/circulationaha.107.732867

2. Bordoni B. The Benefits and Limitations of Evidence-based Practice in Osteopathy. Cureus. 2019. doi:10.7759/cureus.6093

3. Gilham D, Tsujikawa LM, Sarsons CD, et al. Apabetalone downregulates factors and pathways associated with vascular calcification. Atherosclerosis. 2019;280:75-84. doi:10.1016/j.atherosclerosis. 2018.11.002

4. Savinov AY, Salehi M, Yadav MC, Radichev I, Millán JL, Savinova OV. Transgenic Overexpression of Tissue-Nonspecific Alkaline Phosphatase (TNAP) in Vascular Endothelium Results in Generalized Arterial Calcification. Journal of the American Heart Association. 2015;4(12). doi:10.1161/jaha.115.002499

5. Romanelli F, Corbo AM, Salehi M, et al. Overexpression of tissuenonspecific alkaline phosphatase (TNAP) in endothelial cells accelerates coronary artery disease in a mouse model of familial hypercholesterolemia. PLOS ONE. 2017;12(10). doi:10.1371/ journal.pone. 0186426

Financial Disclosures: None reported.

Support: The acquisition of the microCT scanner was made possible by a Major Research Instrumentation (MRI) grant from the National Science Foundation (NSF 1828305) to PIs Simone Hoffmann, Julia Molnar, Azhar Ilyas, Olga Savinova, and Claude Gagna and with the support of NYIT's College of Osteopathic Medicine (NYITCOM), College of Engineering and Computing Sciences, and College of Arts and Sciences. Thank you to Kelsi Hurdle for technical support with collection of the microCT data.
Ethical Approval: Animal studies were approved by the New York Institute of Technology College of Osteopathic Medicine (Old Westbury, NY) and complied with the National Institutes of Health guidelines for humane treatment of laboratory animals.

Informed Consent: Not relevant.

Poster No.: *B20

Abstract No.: 40

Category: Basic Science

Research Focus Area: Osteopathic Philosophy

Can a Digital Ear Otoscope Improve Anatomical Recognition of the Inner Ear Landmarks, Comfort and Confidence in First Year Medical Students?

${ }^{1}$ Elli Kaufmann, OMS-III; ${ }^{1}$ Rawan Sultan, OMS-III; ${ }^{1}$ Dhipi Srinivasan, OMS-III; ${ }^{1}$ Tanya Omar, OMS-III; ${ }^{1}$ Imogen Kane, OMS-III; ${ }^{2}$ Tala Dajani, MD, MPH

${ }^{1}$ A.T. Still University, School of Osteopathic Medicine in Arizona (ATSU-SOMA)

${ }^{2}$ Department of Medical Education, A.T. Still University, School of Osteopathic Medicine in Arizona (ATSU-SOMA)

Context: Current literature has examined the use of digital video otoscopy (DVO) and found it to be as accurate as manual otoscopy when used by a trained medical provider. ${ }^{1,2}$ Another study suggested medical providers have a preference for DVO when identifying tympanic membrane pathology. ${ }^{3}$ It was noted that few studies had been conducted on medical students and their preferences. DVO is a low cost method that may improve student's experiences when learning how to conduct an otoscope exam.

Objective: To identify whether including training in digital video otoscopy (DVO), in addition to training in manual otoscopy examination (MOE), improves ease and accuracy of anatomical recognition for medical students compared to MOE training alone.

Methods: This study was a survey study. 40 medical students self enrolled in this study based on an advertisement posted to the class Facebook group. There was no exclusion criteria other than previous DVO training. Study participants were assigned a number. The participants received a lecture from faculty on how to use the DVO. Participants were randomly paired and assigned to positions of examiner and examinee. The participants then took turns as the examiner and examinee. First, the 
examiner utilized the MOE and then the DVO on the examinee to locate inner ear landmarks and then rate their confidence in locating these landmarks as well as their confidence in using the DVO. Examinees rated their comfort while being examined. Participants then switched roles and repeated the experiment. Data was analyzed by calculating the average score for each category and the standard deviation around that score. The mean scores for DVO were then compared to the mean scores for MOE.

Results: Using data compiled from student surveys, we assessed examinee comfort, examiner comfort, examiner confidence and anatomical landmarks recognition scores in both MOE and DVO. The response rate was $100 \%$, as all students who participated in the study completed a survey as an examinee and an examiner. In first year medical students with 3 months prior experience with MOE and no experience with DVO, examiner anatomy recognition scores for 10 anatomical landmarks were significantly higher with DVO (mean=6.44, $\mathrm{s}=2.83$ ) than MOE (mean=3.15, $\mathrm{s}=2.13)$. Examinee comfort $(\mathrm{mean}=3.95, \mathrm{~s}=1.12$ v. mean $=3.43, s=1.16)$ and examiner confidence (mean=3.82, $\mathrm{s}=1.68 \mathrm{v}$. mean $=3.34, \mathrm{~s}=0.88$ ) were found to have a statistically significant increases with DVO compared to MOE. The Examiner comfort scores trended higher with DVO than MOE but this was not found to be statistically significant in this cohort.

Conclusion: A pilot study with 25 participants done by fellow classmates in 2019 demonstrated a clear preference for DVO. This survey study indicated that DVO was preferred for anatomical recognition of the middle ear, examinees comfort, examiner's comfort, and for examiners' confidence. However, examiner comfort scores were not significant. In this cohort, DVO training was conducted one hour before study intervention. MOE had been taught 3 months prior at the beginning of the semester. Proper and timely training of DVO with MOE during the school year may affect these results and increase student comfort and confidence with DVO. The low-cost options and accessible nature of DVO makes it valuable for providers and patients alike and we hope this can be incorporated in undergraduate medical education and clinical settings in the future.

\section{References}

1. Mousseau S, Lapointe A, Gravel J. Diagnosing acute otitis media using a smartphone otoscope; a randomized controlled trial. Am J Emerg Med. 2018;36(10):1796-1801. doi:10.1016/j.ajem.2018.01. 093

2. MU, Sohal M, Valdez TA, Grindle CR. iPhone otoscopes: Currently available, but reliable for tele-otoscopy in the hands of parents?
Int J Pediatr Otorhinolaryngol. 2018;106:59-63. doi:10.1016/j. ijporl.2018.01.003

3. Sahyouni R, Moshtaghi O, Rajaii R, et al. Evaluation of an iPhone Otoscope in a Neurotrauma Clinic and as an Adjunct to Neurosurgical Education. Insights Neurosurg. 2016;1(1):4. doi:10.21767/ 2471-9633.10004

Financial Disclosures: None reported.

Support: None reported.

Ethical Approval: The study was reviewed and approved on 11/15/2019 by the ATSU-SOMA IRB via the expedited review process. This process was allowed because the research study met the criteria of minimal risk human research and made minor changes to research previously approved by the full board (pilot study done in 2019). The IRB number 2019-198.

Informed Consent: Students were informed of the study through the online facebook group shared with the class. Students self selected if they wanted to participate and were given a consent form to sign before instruction about the study began. The consent form stated the risks and benefits of this study. Each participant was given a consent form and study members were available to answer questions about the study. Questions answered were mainly involving length of time the study would take. All participants who came to the study signed the consent form.

${ }^{\star}$ Poster No.: ${ }^{\text {B } 21}$

Abstract No.: 41

Category: Basic Science

Research Focus Area: Chronic Diseases \& Conditions AOA Grant Award: 19133749

Diet Significantly Influences Physiological and Metabolic Outcomes of a Mouse Model of Cardiovascular Disease

${ }^{1}$ Kayla Boley Corbett MS, OMS-IV; ${ }^{2}$ Claire Saunders; ${ }^{2}$ Andrew Hiatt; ${ }^{2}$ Jeffrey Houghton; ${ }^{2} J o s e p h$ C. Gigliotti, PhD

${ }^{1}$ Liberty University College of Osteopathic Medicine (LUCOM); ${ }^{2}$ Department of Integrative Physiology and Pharmacology, Liberty University College of Osteopathic Medicine (LUCOM)

Context: Diet plays a significant role in health and disease, especially cardiovascular disease (CVD). ${ }^{1}$ Diets common in developed nations are rich in "empty calories," as the intake of energy-yielding nutrients is high while the average intake of several key nutrients is not sufficient ${ }^{2}$. It 
is unclear how these nutritionally inadequate diets influence CVD and its associated risk factors because nutritionally inadequate diets are not available or readily used in mechanistic, preclinical animal studies. ${ }^{3,4}$

Objective: To characterize the effect of different diets on markers of kidney and liver health and metabolic dysfunction in mice with chronic angiotensin II (AngII) infusion, a preclinical model of heart and kidney disease. Methods: Weanling male $\mathrm{C} 57 \mathrm{Bl} / 6$ mice $(\mathrm{N}=24)$ were purchased from the Jackson Laboratory and given 1-week to acclimate to a pelleted chow diet. Mice were then randomly assigned ( $\mathrm{n}=7-9)$ to receive a standard rodent chow (Teklad \#2018), a commercially available Western Diet (WD) (highfat and high-sugar, Teklad \#TD.88137) or our novel American Diet (AD) ad libitum. After 20 weeks on their assigned diet, all mice underwent a brief surgery to implant (subcutaneous) miniosmotic pumps delivering saline (vehicle control, $\mathrm{n}=3$ for each diet) or angiotensin II (AngII, $\mathrm{n}=5$ for each diet) at a dose of $700 \mathrm{ng} / \mathrm{kg}$ body weight per minute. Body weight and blood pressure were recorded each week during the infusion period. Mice remained on their assigned diets throughout the 4-week perfusion study and food intake was measured during the last week by housing mice individually in metabolic cages. Mice were then fasted for 5 hours and euthanized and serum collected for the quantification of circulating triglycerides, glucose, cholesterol, and insulin using commercially available assays (Cayman Chemical). Kidney and liver tissues were collected and processed for routine histology (hematoxylin and eosin) and the quantification of total tissue lipid content. Liver tissues were also processed to quantify the expression of genes involved in hepatic steatosis using commercially available PCR arrays (Qiagen). Data were analyzed using General Linear Models procedures in SPSS (IBM) and Tukey's Post Hoc analysis with statistically significant differences defined as $\mathrm{P}<0.05$.

Results: Diet significantly influenced body weight $(\mathrm{P}<0.001)$, with mice fed the WD being heavier ( $41 \pm 1$ grams) than mice fed the $\mathrm{AD}(30 \pm 1)$ and chow $(27 \pm 1)$. AngII was found to significantly reduce weight gain regardless of caloric intake $(\mathrm{P}=0.008)$, with the magnitude of weight loss dependent upon the diet that was fed $(\mathrm{P}=0.03)$. Diet significantly influenced fasting circulating glucose $(\mathrm{P}=0.03)$, total cholesterol $(\mathrm{P}<0.001)$, and circulating insulin $(\mathrm{P}=0.004)$, with mice fed the WD having the highest fasting glucose $(287 \pm 26 \mathrm{mg} / \mathrm{dL})$, total cholesterol $(232 \pm 15 \mathrm{mg} / \mathrm{dL})$, and insulin concentrations $(3 \pm 0.5 \mathrm{ng} / \mathrm{mL})$. AngII did not significantly influence any of the metabolic parameters measured. Mice fed the $\mathrm{AD}$ had the greatest kidney weights $(0.40 \pm 0.01$ grams, $\mathrm{P}=0.04)$ as compared to mice fed chow $(0.35 \pm 0.01)$ but not WD $(0.37 \pm 0.01)$. Kidney lipid vacuolization and increased kidney lipid content was observed in mice fed the WD and $\mathrm{AD}$, with the $\mathrm{AD}-\mathrm{fed}$ mice having the greatest evidence despite having a significantly lower body weight than mice fed WD $(\mathrm{P}<0.001)$. Mice fed the WD had the greatest liver weight ( $2.4 \pm 0.2$ grams) as compared to mice fed $\mathrm{AD}$ $(1.1 \pm 0.2)$ and chow $(1.0 \pm 0.2)$. Hepatic steatosis and increased liver lipid content was observed in mice fed WD $(\mathrm{P}<0.001)$, with minimal change in mice fed $\mathrm{AD}$ and chow. AngII did not significantly influence either kidney $(\mathrm{P}=0.3)$ or liver weights $(\mathrm{P}=0.9)$ or tissue lipid content $(\mathrm{P}=0.4)$. Mice fed chow had a slower development of hypertension in response to AngII as compared to mice fed WD or $\mathrm{AD}(\mathrm{P} \leq 0.05$ at week 2); however, the effect of AngII waned in mice fed AD and WD during the 4th week. 46-genes related to hepatic lipid metabolism and deposition were identified as having a greater than 2-fold change in response to diet and/or AngII. With validation, 10 genes were found to be significantly altered by diet while only 6 were significantly altered by AngII.

Conclusion: The findings of the current study highlight the ability of diet to significantly alter the outcomes of an animal model of CVD. A high calorie WD resulted in overt metabolic dysfunction, while our novel AD (developed to model "typical" American nutritional inadequacies) developed an intermediate metabolic dysfunction as compared to mice fed WD and chow. Interestingly, mice fed the $\mathrm{AD}$ displayed a greater degree of lipid vacuolization despite having a similar liver lipid content as mice fed chow. This alteration in kidney structure could explain why, despite a lower degree of metabolic dysfunction, the mice fed AD had an increased susceptibility to hypertension in response to chronic AngII infusion as observed in mice fed the WD. Additional studies are needed to confirm these findings in other models of CVD and determine the physiological significance of renal lipid vacuolization in mice fed the AD.

\section{References}

1. Mozaffarian D. Dietary and Policy Priorities for Cardiovascular Disease, Diabetes, and Obesity. Circulation. 2016;133(2):187-225. https://doi.org/10.1161/CIRCULATI ONAHA.115.018585. doi:10. 1161/CIRCULATIONAHA.115.018585.

2. USDA Agricultural Research Services 2019. Usual Nutrient Intake from Food and Beverages, by Gender and Age, What We Eat in America, NHANES 2013-2016. http://www.ars.usda.gov/nea/ bhnrc/fsrg. Updated 2021.

3. Lai M, Chandrasekera PC, Barnard ND. You are what you eat, or are you? The challenges of translating high-fat-fed rodents to human obesity and diabetes. Nutr Diabetes. 2014;4(9):e135. doi:10.1038/ nutd.2014.30 [doi]. 
4. Hintze KJ, Benninghoff AD, Cho CE, Ward RE. Modeling the Western Diet for Preclinical Investigations. Adv Nutr. 2018;9(3):263-271. doi:10.1093/advances/nmy002 [doi].

Financial Disclosures: None reported.

Support: J.C.G is funded by grants from Liberty University College of Osteopathic Medicine, National Institutes of Health (R01DK113632-01A1, Co-investigator), and the American Osteopathic Association (\#19133749, Principal Investigator)

Ethical Approval: All animal experiments were conducted in accordance with policies of the National Institutes of Health (NIH) Guide for the Care and Use of Laboratory Animals and the Liberty University Institutional Animal Care and Use Committee (protocol \#13.170421).

Informed Consent: N/A

${ }^{\star}$ Poster No.: ${ }^{\star B} 22$

Abstract No.: 44

Category: Basic Science

Research Focus Area: Chronic Diseases \& Conditions

Novel Micro-CT Method to Assess Atherosclerotic Calcification in Mice

${ }^{1}$ Jashandeep Kaur, OMS-II; ${ }^{2}$ Beatrice Carpo; ${ }^{2}$ Nina D. Kosciuszek; ${ }^{2}$ Olga V. Savinova

${ }^{1}$ New York Institute of Technology (NYITCOM); ${ }^{2}$ Department of Medicine, New York Institute of Technology (NYITCOM)

Context: Atherosclerosis has a common feature of calcification that can lead to restriction of blood flow to organs and tissues. Using animal models such as mice, we can study the mechanisms of calcification in atherosclerosis as a step toward prevention in humans. In animal models, we typically measure vascular calcification using laborious histological methods. In this study, we developed a microCT method to quantify calcium volume in the aortic roots and arches in mice with atherosclerosis.

Objective: The purpose of this study was to evaluate feasibility and compare ex-vivo calcium measurements between the micro-CT and histological methods qualitatively and quantitatively.

Methods: To develop this novel technique, we used a mouse model of increased atherosclerotic calcification. This mouse model utilized overexpression of tissue nonspecific alkaline phosphatase (TNAP) in macrophages, which promotes vascular calcification. TNAP regulates pyrophosphate levels, which inhibits mineralization. Upregulation of TNAP leads to pathological calcification in animal models. Based on the osteopathic principle of the interrelationship of structure and function, any disruption in cardiac function affects not only related organ systems, but also the body's physiology overall. Establishing this relationship may allow for future research on the efficacy of osteopathic techniques, such as myofascial release and lymphatic pump for regulating circulation, on patients with atherosclerosis. Using the micro-CT method, we can visualize this calcification in an uncomplicated approach. Micro-CT images of whole hearts and aortic arches were collected at a 7.1-micron resolution using the SkyScan 1173. Multiple Deep Learning models, implemented in Dragonfly 2020.1 (ORS), were trained on one sample for image segmentation. Standard histological analysis was performed using stains such as H\&E and alizarin red under microscopy to correlate findings with the micro-CT output.

Results: This section details the protocol developed.

Sample preparation: Hearts and aortic arches were dissected from formalin-fixed mice. Hearts were perfused and immersed in mineral oil. Up to three (3) whole hearts or nine (9) aortic arches were assembled for scanning in a $4 \mathrm{~mL}$ standard cryogenic sample vial (Corning). Cotton and parafilm were used as spacers between each sample.

Scanning conditions: SkyScan 1173 was used for microCT imaging. $0.5 \mathrm{~mm}$ custom aluminum filter was used. The voltage was $60 \mathrm{kV}$, current was $133 \mu \mathrm{A}$, resolution was $2 \mathrm{~K}$, and pixel size was $7.144293 \mu \mathrm{M} .1800$ projections were collected. The images were reconstructed via Bruker.

Image Reconstruction: Multiple preparations of the heart and aortic roots can be scanned in a single overnight experiment. We utilized the segmentation wizard feature in Dragonfly 2020.1 (ORS) to select and train a Deep Learning Model. Micro-CT images were classified into three regions of interest: background, soft tissue, and calcification. The range used was between -1000 to 1300 as it corresponds to the Hounsfield units used in micro-CT. Then the images were tested on various models of segmentation. It was found that the Deep Learning model with U-Net5 architecture was most successful in the segmentation of soft tissues and calcifications, while excluding micro-CT artifacts, such as rings and streaks. A subset of samples was also processed by standard histological methods and showed a positive correlation with micro-CT analysis. According to the micro-CT analysis, TNAP transgenic mice had a 4-fold increase in calcification volume in the aortic root compared to control mice.

Conclusion: Our micro-CT method is feasible and, in some aspects, superior to the traditional histological analysis because it is less time-consuming and less prone to artifacts. Moreover, our micro-CT approach is non-destructive 
thus permits analyses of tissue samples by multiple analytical methods after the removal of mineral oil.

\section{References}

1. Frostegård J. Immunity, atherosclerosis and cardiovascular disease. BMC Med. 2013;11:117. Published 2013 May 1. doi:10.1186/ 1741-7015-11-117

2. Romanelli F, Corbo A, Salehi M, et al. Overexpression of tissuenonspecific alkaline phosphatase (TNAP) in endothelial cells accelerates coronary artery disease in a mouse model of familial hypercholesterolemia. PLoS One. 2017;12(10):e0186426. Published 2017 Oct 12. doi:10.1371/journal.pone.0186426

3. Rogers JT, Rogers JC. The role of osteopathic manipulative therapy in the treatment of coronary heart disease. J Am Osteopath Assoc. 1976;76(1):21-31.

4. Borland SJ, Behnsen J, Ashton N, et al. X-ray Micro-Computed Tomography: An Emerging Technology to Analyze Vascular Calcification in Animal Models. Int J Mol Sci. 2020;21(12):4538. Published 2020 Jun 25. doi:10.3390/ijms21124538

5. Mosch J, Gleissner CA, Body S, Aikawa E. Histopathological assessment of calcification and inflammation of calcific aortic valves from patients with and without diabetes mellitus. Histol Histopathol. 2017;32(3):293-306. doi:10.14670/HH-11-797

Financial Disclosures: None reported.

Support: None reported.

Ethical Approval: Not applicable to our study.

Informed Consent: Not applicable to our study.

Poster No.: *B23

Abstract No.: 45

Category: Basic Science

Research Focus Area: Chronic Diseases \& Conditions

Characterization of corpora amylacea in the spinal cord and hippocampal formation of aged individuals

${ }^{1}$ Trevor Pritchett, OMS-II; ${ }^{2}$ Josh Rosenberger, OMS-II; ${ }^{2}$ Joyce Morris-Wiman, $\mathrm{PhD}$

${ }^{1}$ West Virginia School of Osteopathic Medicine (WVSOM); ${ }^{2}$ Department of Biomedical Sciences, West Virginia School of Osteopathic Medicine (WVSOM)

Context: Studies suggest that cerebral corpora amylacea (CCA) may function to scavenge cellular debris that are then eliminated through the glymphatic system. Spinal cord corpora amylacea (SCCA) have been previously reported to contain tau and ubiquitin, suggesting that, as in the cerebrum, SCCA function in eliminating waste. The composition and location of SCCA has led us to hypothesize that SCCA 'waste containers' would be associated with lymphatic or vasculature structures for elimination.

Objective: In this study, to further characterize CA, we have compared diameters of CCA to diameters of SCCA and $\mathrm{CA}$ in the sciatic nerve. We also investigated the association of SCCA and CCA with vascular and lymphatic structures within the cerebrum and spinal cord to provide support for the hypothesis that CA function in the elimination of waste products from the spinal cord.

Methods: Subjects: Spinal cord and sciatic nerve specimens were harvested from six female donors to the WVSOM Human Gift Program, ages 81-95, and from the medial temporal lobe of a female $83 \mathrm{yr}$. old donor. The tissue was cryoprotected, snap-frozen in isopentane cooled in liquid nitrogen, and stored at $-80^{\circ}$ until cryosectioned at $14 \mu \mathrm{M}$.

Analysis of CA distribution and size: Cryosections were stained using an alcoholic PAS method to identify CA. Digital images of spinal cord, sciatic nerve and hippocampus were acquired using a Leica Aperio system and analyzed using ImagePro Plus software. Briefly, images were first converted to 8-bit grayscale images and thresholded to produce a binary image in which CA could be counted and measured. Statistical comparisons between groups and were made using a two way ANOVA (MiniTab 20).

Analysis of colocalization of laminin and lyve1 and PAS inclusions: Cryosections were immunostained for laminin and lyve1+using standard protocols. Briefly, sections were incubated in primary antibody (antilaminin or anti-lyve1) overnight at 40C. Sections were then incubated in appropriate secondary antibody for $2 \mathrm{hrs}$. Images were acquired under epifluorescence using the Leica Aperio system. After soaking in PBS to remove coverslips and glycerol mount, sections were processed for PAS-staining as described above to delineate CA. The corresponding immunostained image was converted to an 8-bit image and thresholded to delineate vascular or lymphatic elements. The PAS binary image was then overlaid by its corresponding binary immunostained image and position of CA within the dorsal column and horn of the spinal cord, or within a delineated region of the hippocampus or sciatic nerve, characterized as either within or touching a vessel or single. Statistical comparisons between groups and 
were made using a two way ANOVA and, when significant $(\mathrm{p}<0.05)$, were examined pair-wise using post-hoc comparisons (Minitab 20).

Results: CA average diameter and distribution: As in previous studies, CA numbers were observed to be enriched within spinal cord dorsal regions, although inclusions could be identified in all regions of the spinal cord. CA in the medial temporal lobe were limited mainly to regions of the hippocampal cortex, the lateral ventricle and dural covering. CA were identified within the sciatic nerve, although their numbers were limited. The average area of CA in the spinal cord $\left(117.1 \mu \mathrm{m}^{\wedge} 2\right)$ was not statistically different from that observed for CA in the hippocampus $\left(124.4 \mu \mathrm{m}^{\wedge} 2\right)$. However, the CA detected within the sciatic nerve $\left(151.2 \mu \mathrm{m}^{\wedge} 2\right)$ were statistically larger than those in the spinal cord or cerebrum (ANOVA, LSD post-hoc test).

Location of CA within vasculature and lymphatics: Our analysis was limited to the dorsal spinal cord, as our previous study had shown that this spinal cord region was enriched in CA. Also studies of lymphatic drainage in the spinal cord suggest that much of the drainage is into the dorsal vessels. Because lyve1+ immunostaining did not adequately delineate lymphatic vessels, but had a patchy expression that appeared to parallel that of laminin, we only used laminin immunostaining to characterize CA position within the spinal cord and hippocampus. The lymphatics of the spinal cord are not well described, but studies suggest that lymphatic vessels are associated with small blood vessels. Only $31 \%$ of CA identified in the dorsal spinal cord were associated with laminin immunopositive structures; most were in the most peripheral regions of the dorsal spinal cord. $32 \%$ of CA detected within the hippocampus were associated with vessels. There were no statistical differences CA/vessel distribution between the dorsal spinal cord and the hippocampus (ANOVA).

Conclusion: The results of this study indicate that CA within the spinal cord of aged individuals, identified as PAS-positive inclusion bodies, are of a similar size to corpora amylacea described in the cerebral hemispheres of aged individuals. Their location within the spinal cord periphery, most often associated with vascular/lymphatic structures, and our previous observation of tau and ubiquitin within the inclusions, suggest that, as in the cerebrum, CA in the spinal cord function in eliminating debris.

\section{References NA}

Financial Disclosures: None reported.

Support: This project was funded by a WVSOM Intramural Grant and by WVSOM Summer Student Research.

Ethical Approval: Exempt

Informed Consent: NA

Poster No.: *B24

Abstract No.: 52

Category: Basic Science

Research Focus Area: Chronic Diseases \& Conditions

AOA Grant Award: \#19133749

\section{Investigating the Prophylactic and Therapeutic Effect of IL233 Administration on Angiotensin II-Induced Hypertension in 129SVEV and C57BL/6 Mice}

${ }^{1}$ Spencer Neaville, OMS-III; ${ }^{1}$ Cerena Merchant; ${ }^{1}$ Alyssa Kang; ${ }^{2}$ Victoria Louise Krause; ${ }^{2}$ Vikram Sabapathy; ${ }^{2}$ Rahul Sharma; ${ }^{3}$ Joseph Gigliotti

${ }^{1}$ Liberty University College of Osteopathic Medicine (LUCOM); ${ }^{2}$ Department of Medicine, University of Virginia School of Medicine; ${ }^{3}$ Department of Integrated Physiology and Pharmacology, Liberty University College of Osteopathic Medicine (LUCOM)

Context: Hypertension (HTN) is a leading cause of disease and death worldwide. ${ }^{1}$ While much of its etiology remains unknown, the immune system is believed to play a key role in the etiology of HTN and its progression to end organ damage. $^{2}$ The cytokines interleukin 2 (IL-2) and IL-33 synergistically decrease inflammation and organ damage in animal models of human disease, and recently a hybrid IL2-IL33 cytokine (IL233) was developed. ${ }^{3}$ Therefore, it is plausible that IL233 may be a therapy to reduce HTN.

Objective: To determine if prophylactic or therapeutic administration of IL233 may reduce the severity of inflammation-induced end organ damage secondary to AngII-induced hypertension in mice.

Methods: 8-week-old male C57Bl/6 mice were used due to their routine usage as a preclinical mouse model with AngII-HTN, while the 129SVEV (129) strain was chosen due 
to its increased susceptibility to cardiovascular and renal injury. In the prophylactic experiment, 129 and C57BL/6 mice received 2ug IL233 ( $\mathrm{n}=7$ and 4, respectively) or saline control ( $\mathrm{n}=7$ and 4) via intraperitoneal injection daily for five consecutive days. Miniosmotic pumps delivering $800 \mathrm{ng} / \mathrm{kg}^{\star} \min (\mathrm{n}=16)$ were then implanted subcutaneously. Systolic blood pressure (SBP) was measured every 48-hours using a tail-cuff system. After 2-weeks, 129 mice were placed in individual metabolic cages to measure food and water consumption and collect urine for quantification of albuminuria using a commercially available ELISA. 129 mice were then euthanized, and kidney and splenic leukocyte content was quantified by flow cytometry (FC). After 2-weeks of AngII infusion, 24-hour food intake and body weight were recorded in $\mathrm{C} 57 \mathrm{BL} / 6$ mice and renal blood flow (RBF) was then quantified using ultrasound. In the therapeutic experiment, 129 mice were administered AngII via subcutaneous osmotic pumps at a dose of $800 \mathrm{ng} / \mathrm{kg}$ per day. Once HTN developed (day 6), mice received either IL233 $(n=4)$ or saline control $(n=3)$. Blood pressure was recorded for 28 days after pump implantation and mice were then housed individually in metabolic cages to quantify fluid balance and collect urine. Mice were then euthanized, and kidney and spleen tissues were analyzed by FC. All data were analyzed using General Linear Models procedures in SPSS (IBM). Blood pressure was analyzed using repeated measures analysis and $\mathrm{P}<0.05$ was considered statistically significant.

Results: The 129 mice given prophylactic injections of IL233 had greater 24-hour water intake $(5.6 \pm 1 \mathrm{~mL})$ as compared to mice administered saline $(4.1 \pm 0.7, \mathrm{P}=0.04)$ which caused increased urinary output in mice administered IL233 after receiving AngII ( $2.1 \pm 0.7$ vs $0.8 \pm 0.2 \mathrm{~mL}$, $\mathrm{P}=0.009)$. However, prophylactic IL233 treatment did not prevent AngII-induced hypertension in the $129(133 \pm 10$ vs $146 \pm 10 \mathrm{mmHg}$, saline and IL233 respectively, $\mathrm{P}=0.45$ ) or C57BL/ 6 mouse strains ( $120 \pm 3$ vs $119 \pm 3$, saline and IL233 respectively, $\mathrm{P}=0.75)$. Furthermore, there was no effect of IL233 on body $(\mathrm{P}=0.26)$ or heart weights $(\mathrm{P}=0.25)$ or albuminuria $(\mathrm{P}=0.24)$. Flow cytometry analysis showed that prophylactic IL233 treatment significantly decreased tumor necrosis factor alpha (TNF $\alpha$ ) production by splenic CD4+ $\mathrm{T}$-cells compared to saline vehicle $(\mathrm{P}=0.009)$ and there was also a tendency $(\mathrm{P}=0.06)$ for decreased splenic Treg cells in mice that received prophylactic injections of IL233, which differs from an increase in Tregs and accompanied decline in TNF $\alpha$ levels in all the other inflammatory disease models. IL233-treated C57BL/6 mice had a numerical increase in RBF at both baseline and after saline administration, although neither reached statistical significance $(\mathrm{P}=0.1$ and 0.16 , respectively). In the therapeutic group, there was no overall difference in SBP between mice given saline (132 $\pm 5 \mathrm{mmHg})$ or IL233 (137 $\pm 5 \mathrm{mmHg})$, although IL233 treated mice had a heightened $(\mathrm{P}=0.049)$ reaction to AngII SBP at week $3(151 \pm 15 \mathrm{mmHg})$ as compared to mice receiving control injections (118 \pm 22$)$. After 4 weeks of AngII infusion, there was no difference in water intake $(\mathrm{P}=0.8)$ or urinary output $(\mathrm{P}=0.9)$ between saline and IL233 treated mice. Cytology testing from UVA showed no significant differences between the groups regarding kidney inflammation as well as splenic and circulating markers.

Conclusion: In male C57Bl/6 and 129 mice, we found that IL233 did not prevent HTN or kidney injury in the in mice with chronic AngII infusion. Prophylactic IL233 resulted in greater urinary output in the 129 strain, implying that IL233 may influence pressure diuresis. IL233 significantly reduced TNF $\alpha$ production by splenic CD4+ cells IL233 was effective in modulating the immune system. However the significance of this finding in the context of HTN remains unclear. Similarly, therapeutic IL233 injections had no significant protection against AngII-HTN in 129 mice. Our data suggests that IL233 administered at a dose of 2 ug per day is not effective in reducing AngII-HTN in mice. Future studies are needed to determine if higher doses of IL233 are needed, and if IL233 is effective in other animal models of HTN. Although much of the effects and physiologic mechanism of IL233 remain to be discovered, this novel cytokine continues to show promise as a future therapeutic.

\section{References}

1. Murray CJL, Aravkin AY, Zheng P, et al. Global burden of 87 risk factors in 204 countries and territories, 1990-2019: a systematic analysis for the Global Burden of Disease Study 2019. The Lancet. 2020;396(10258):1223-1249. https://doi.org/10.1016/S01406736(20)30752-2. doi:10.1016/S0140-6736(2030752-2).

2. Norlander AE, Madhur MS, Harrison DG. The immunology of hypertension. J Exp Med. 2018;215(1):21-33. doi:10.1084/jem. 20171773 [doi].

3. Stremska ME, Jose S, Sabapathy V, et al. IL233, A Novel IL-2 and IL-33 Hybrid Cytokine, Ameliorates Renal Injury. J Am Soc Nephrol. 2017;28(9):2681. http://jasn.asnjournals.org/content/28/9/2681. abstract. doi:10.1681/ASN.2016121272.

Financial Disclosures: The IL233 IP is licensed by SlateBio Inc, where RS is an equity holder.

Support: J.C.G is funded by grants from Liberty University College of Osteopathic Medicine, National Institutes of Health (R01DK113632-01A1, Co-investigator), and the American Osteopathic Association (\#19133749, Principal Investigator). RS and VS are supported by NIH/NIDDK 
awards to RS (1R01DK104963 and 1R01DK105833, Principle Investigator).

Ethical Approval: All animal studies were performed according to animal protocols approved by the Liberty University Institutional Animal Care and Use Committee (protocol \#59).

Informed Consent: None.

${ }^{\star}$ Poster No.: ${ }^{\star B} 25$

Abstract No.: 58

Category: Basic Science

Research Focus Area: Chronic Diseases \& Conditions

Evaluating the structure and

function of cysteine residues of the

Pseudomonas aeruginosa nucleotidyl cyclase type three secreted effector, ExoY

\author{
${ }^{1}$ Katherine Xenna Goh, OMS-II; ${ }^{2}$ Marc Benson, PhD \\ ${ }^{1}$ West Virginia School of Osteopathic Medicine (WVSOM); \\ ${ }^{2}$ Department of Biomedical Sciences, West Virginia School \\ of Osteopathic Medicine (WVSOM)
}

Context: Pseudomonas aeruginosa is a bacterium associated with aggressive infections in immunocompromised and hospitalized patients. ExoY is a type three secreted virulence factor that possesses nucleotidyl cyclase activity. The relationship between its structure and its enzymatic function in host cells remains unclear as ExoY bundles actin filaments independent of nucleotidyl cyclase activity. ${ }^{1}$ This study highlights our initial research to better understand these two different activities in vitro.

Objective: This project analyzes whether the amino acid structure-specifically five cysteine residues-alters the structure and/or function of ExoY. If mutations of the cysteine residues do not alter nucleotidyl cyclase activity and lower aberrant disulfide bond formation in vitro, mutagenized forms of the protein may be utilized for future research. By understanding how ExoY elevates cGMP in host cells, a potential gene therapy for diseases with low guanylyl cyclase activity could be developed.

Methods: Site-directed mutagenesis of the five cysteine residues to alanine was previously performed. Plasmids included the exoY gene with one of five cysteine codons mutagenized to an alanine codon; C100A, C205A, C279A, C331A, or C345A. Plasmids with the mutagenized exoY gene were transformed into E. coli BL21 for the expression of recombinant protein. Overnight cultures were subcultured in LB medium with kanamycin and grown to an OD600 of 0.4-0.6. Expression of ExoY was induced with the addition of isopropyl $\beta$-D-1 thiogalactopyranoside (IPTG). The culture was incubated at room temperature for 4 hours then centrifuged at 10,000 xg for 5 mins to collect the cell pellet. B-PER (Bacterial Protein Extraction Reagent) supplemented with protease inhibitors, lysozyme, DNase, and RNase was added to the pellet to lyse the cells. The supernatant was collected by centrifugation at $10,000 \mathrm{xg}$ for 10 minutes. Soluble material (supernatant) was collected by ultracentrifugation at 100,000 xg for one hour. Equal parts of $2 \mathrm{x}$ nickel resin binding buffer was added to the supernatant and passaged through nickel resin. The histidine-tagged ExoY proteins were eluted using $500 \mathrm{mM}$ imidazole, concentrated and resolved by size-exclusion chromatography. Protein concentration was determined using SDS-PAGE densitometry. ExoY guanylyl cyclase activity was measured using the cGMP Parameter assay kit (R\&D Systems, Minneapolis, MN). Structural changes were detected using limited proteolysis; Coomassie-stained bands from the protease-digested mutagenized ExoY were compared to the protease-digested wild type ExoY.

Results: As wild-type ExoY precipitates in solution without a reducing agent, we examined the solubility of the five cysteine mutagenized ExoY proteins. After purification, the mutagenized proteins were incubated at $4^{\circ} \mathrm{C}$ in the presence or absence of a reducing agent. All proteins without reducing agent precipitated within two weeks while all remained soluble in the presence of reducing agent, suggesting that no single cysteine residue, when mutagenized to alanine, abrogates aberrant disulfide formation. To determine if a single cysteine residue contributed to ExoY activity, cGMP production for each cysteine-mutagenized protein was examined. Preliminary results suggest that nucleotidyl cyclase activity among the mutagenized ExoY proteins is comparable, all producing cGMP in the range of 1,500-2,500 pmoles. This suggests that no single cysteine residue plays a role in cyclase activity in vitro. To examine how each cysteine residue contributes to the structure of ExoY, limited proteolysis was performed for each cysteine mutation and the resulting proteolytic fingerprint was compared to the wild-type protein. The ExoY C100A and C205A proteins have similar proteolytic fingerprints when compared to wild-type, suggesting that these cysteines play no role in the overall structure of ExoY in vitro. Structural and catalytic analysis of the mutagenized ExoY proteins is ongoing.

Conclusion: Findings from this study contribute to our communal understanding of P. aeruginosa pathogenesis through the examination of the structural and enzymatic activities of ExoY. Of the four type III secretion toxins secreted 
by P. aeruginosa, ExoY is the only effector that possesses cysteine residues, forming aberrant disulfide bonds that cause the recombinant protein to precipitate in solution. Recent studies have also shown that ExoY has a preference for guanosine 3',5'-cyclic monophosphate (cGMP) production over cAMP, making it a potential therapy for diseases with dysregulated levels of cGMP like hypertension. ${ }^{2,3}$ Based on ExoY's crystal structure, no disulfide bridge forms between any of the cysteine residues and cyclase activity in unchanged in the presence of reducing agents, suggesting that these cysteines do not form disulfide bridges. ${ }^{4}$ Our results confirm that the cysteine residues within ExoY do not form disulfide bridges or seem to be necessary for cyclase activity in vitro. Although activity is unaffected by cysteine mutations, we examined how the $\mathrm{C} 100 \mathrm{~A}$ and $\mathrm{C} 205 \mathrm{~A}$ mutations may affect the structure of the protein. We found that neither of these cysteines play a structural role in vitro. If all five cysteines can be replaced by the less reactive amino acid, alanine, we speculate that ExoY can be produced recombinantly and be more stable in solution, allowing for downstream biochemical and pharmacological studies to be performed. This analysis provides a preparatory foundation for future working examining how ExoY alters the host cell cytoskeleton independent of cyclase activity. From our work, perhaps a novel therapeutic that targets disease states associated with low cGMP levels could be elucidated.

\section{References}

1. Mancl JM, Suarez C, Liang WG, Kovar DR, Tang W-J. Pseudomonas aeruginosa exoenzyme $Y$ directly bundles actin filaments. J Biol Chem. 2020;295(11):3506-3517. doi:10.1074/jbc.RA119.012320

2. Beckert $\mathrm{U}$, Wolter $\mathrm{S}$, Hartwig $\mathrm{C}$, et al. ExoY from Pseudomonas aeruginosa is a nucleotidyl cyclase with preference for cGMP and cUMP formation. Biochem Biophys Res Commun. 2014;450(1):870874. doi:10.1016/j.bbrc.2014.06.088

3. Ataei Ataabadi E, Golshiri K, Jüttner A, Krenning G, Danser AHJ, Roks AJM. Nitric Oxide-cGMP Signaling in Hypertension: Current and Future Options for Pharmacotherapy. Hypertension. 2020;76(4):1055-1068. doi:10.1161/HYPERTENSIONAHA.120.15856

4. Khanppnavar B, Datta S. Crystal structure and substrate specificity of ExoY, a unique T3SS mediated secreted nucleotidyl cyclase toxin from Pseudomonas aeruginosa. Biochim Biophys Acta Gen Subj. 2018;1862(9):2090-2103. doi:10.1016/j.bbagen.2018.05.021

Financial Disclosures: None reported.

Support: Research reported in this publication was supported by the National Institute of General Medical Sciences of the National Institutes of Health under award number P20GM103434. The content is solely the responsibility of the authors and does not necessarily represent the official view of the National Institutes of Health; West Virginia Higher Education Policy Commission Division of Science and Research; West Virginia School of Osteopathic Medicine.

Ethical Approval: N/A

Informed Consent: N/A

Poster No.: *B26

Abstract No.: 61

Category: Basic Science

Research Focus Area: Chronic Diseases \& Conditions

Connexin 43 Up-regulation by AMPK Deficiency in Cardiomyocytes Treated with the Anticancer Drug Doxorubicin

${ }^{1}$ Mandeep Singh, OMS-II; ${ }^{2}$ Naunihal Singh, BS, OMS II; ${ }^{2}$ Tamayo Kobayashi; ${ }^{2}$ Saturo Kobayashi, PhD; ${ }^{2}$ Qiangrong Liang, MD, PhD

${ }^{1}$ New York Institute of Technology (NYITCOM); ${ }^{2}$ Department of Biomedical Sciences, New York Institute of Technology (NYITCOM)

Context: Doxorubicin (DOX) is an anticancer drug that can cause heart failure. ${ }^{1}$ DOX cardiotoxicity has been attributed to ROS production and genotoxicity, leading to cell death. ${ }^{2}$ AMPK, a kinase, has been proposed to modulate DOX-induced cardiotoxicity ${ }^{3}$, but the downstream mediators remain unclear. Connexin $43(\mathrm{Cx} 43)$ is a gap junction protein needed for heart function. ${ }^{4}$ Studies have suggested the role of Cx43 in DOX cardiotoxicity, but whether the response is through AMPK remains unclear. Objective: To determine the role of AMPK in the regulation of the $\mathrm{Cx} 43$ in response to DOX-induced cardiotoxicity.

Methods: H9c2 rat cardiomyocytes were seeded on 6-well plates and cultured in DMEM with 6.5\% fetal bovine serum. To investigate the role of AMPK, the cells were transfected with siRNAs targeting different AMPK isoforms using Lipofectamine RNAiMAX and Opti-MEM I reduced serum medium. After 48 hours of transfection, half of the wells received $0.5 \mu \mathrm{M}$ DOX treatment, and the other half received equal amounts of saline. Protein samples were collected 16 hours later using the Cell lysis buffer. Western blot analysis was performed to confirm the knockdown of AMPK isoforms and determine the protein expression levels of $\mathrm{Cx} 43$. 
Results: Our findings showed that siRNA transfections effectively reduced the protein levels of AMPK isoform a1 and a2, respectively, as indicated by the Western blot analysis. DOX treatment increased the expression levels of the $\mathrm{Cx} 43$ in cardiomyocytes. Also, knockdown (KD) of AMPK $\alpha 1$ isoform resulted in a similar increase in $\mathrm{Cx} 43$ expression levels as did DOX, but AMPK $\alpha 2 \mathrm{KD}$ did not significantly alter Cx43 levels, suggesting an AMPKa1 specific effect on $\mathrm{Cx} 43$ expression. Interestingly, AMPKa1 $\mathrm{KD}$, together with DOX treatment, did not produce a synergistic effect to increase Cx43 expression levels further, suggesting the possibility that DOX might have elevated the $\mathrm{Cx} 43$ expression through its effect on AMPK. Conclusion: Our results confirmed the previous studies showing that treatment of the cardiomyocytes with DOX increased the expression of Cx43. However, our investigations produced a novel finding that knocking down AMPK $\alpha 1$ also increased the $\mathrm{Cx} 43$ but did not enhance the effect of DOX on Cx43 expression, suggesting a role for AMPK in the pathway that regulates the $\mathrm{Cx} 43$ to mediate the DOX-induced cardiotoxicity. Our investigations also showed an isoform-specific effect of AMPK on Cx43 expression since only $\alpha 1$ but not $\alpha 2 \mathrm{KD}$ increased Cx43 levels. Whether the increase in $\mathrm{Cx} 43$ is due to increased gene expression or inhibited, protein degradation remains to be determined. Further investigation is also warranted to elucidate the roles of other AMPK subunits or isoforms in regulating the Connexin 43 expression in the context of doxorubicin cardiotoxicity.

\section{References}

1. Singal PK, Iliskovic N. Doxorubicin-induced Cardiomyopathy. New England Journal of Medicine. 1998;339(13):900-905. doi:10.1056/nejm199809243391307. https://doi.org/10.1056/ nejm199809243391307

2. Octavia Y, Tocchetti CG, Gabrielson KL, Janssens S, Crijns HJ, Moens AL. Doxorubicin-induced cardiomyopathy: from molecular mechanisms to therapeutic strategies. I Mol Cell Cardiol. 2012;52(6):1213-1225. doi:10.1016/j.yjmcc.2012.03.006. https:// doi.org/10.1016/j.yjmcc.2012.03.006

3. Timm KN, Tyler DJ. The Role of AMPK Activation for Cardioprotection in Doxorubicin-Induced Cardiotoxicity. Cardiovasc Drugs Ther. 2020;34(2):255-269. doi:10.1007/s10557-020-06941-x. https:// doi.org/10.1007/s10557-020-06941-x

4. Prakoura N, Kavvadas P, Chadjichristos CE. Connexin 43: a New Therapeutic Target Against Chronic Kidney Disease. Cell Physiol
Biochem. 2018;49(3):985. doi:10.1159/000493230. https://doi. org/10.1159/000493230

Financial Disclosures: This study is supported by $\mathrm{NIH}$ grant 1R15HL137130-01A1.

Support: None reported.

Ethical Approval: NA

Informed Consent: NA

Poster No.: *B28

Abstract No.: 64

Category: Basic Science

Research Focus Area: Chronic Diseases \& Conditions

AOA Grant Award: \#19133749

\section{Determining the Physiological, Immunological, and Metabolic Consequences of Chronic Angiotensin II Infusion in Mice}

${ }^{1}$ Pranav Reddy Bommineni, OMS-IV; ${ }^{2}$ Henry Miller; ${ }^{2}$ Jeffrey Houghton; ${ }^{2}$ Joseph C. Gigliotti, PhD

${ }^{1}$ Liberty University College of Osteopathic Medicine (LUCOM); ${ }^{2}$ Department of Integrative Physiology and Pharmacology, Liberty University College of Osteopathic Medicine (LUCOM)

Context: Angiotensin II (AngII) is a pleiotropic hormone best known for regulating fluid balance and blood pressure. ${ }^{1}$ Recent work suggests that AngII also modulates the immune system ${ }^{2,3}$ and metabolism ${ }^{4,5}$, however the details of these interactions are unknown. Chronic AngII infusion is a common mouse model of cardiovascular and kidney injury, and it is plausible that alterations in the immune response and energy utilization may also contribute to the phenotype observed in this important animal model.

Objective: To determine the effect of chronic AngII infusion in mice (a popular preclinical animal model of cardiovascular and kidney injury) on systolic blood pressure (SBP), renal health, and weight gain.

Methods: 8-week, male $\mathrm{C} 57 \mathrm{Bl} / 6$ mice $(\mathrm{N}=20)$ were purchased from the Jackson Laboratory and given 14-days to acclimate to the new environment and to collect baseline SBP. Mice were then randomly assigned $(n=5)$ to receive 0 (saline vehicle), 400, 800 , or $1200 \mathrm{ng} / \mathrm{kg}^{\star}$ minute AngII via miniosmotic pump implanted in a subcutaneous 
space in the rear flank. Mouse SBP and body weights were recorded each week during the 4-week infusion period. During the 4th-week, mice were individually housed in metabolic cages to collect urine and assess food intake. Urinary albumin concentration (a marker of renal injury) was quantified with a commercially available assay. Mice were then euthanized, and tissues collected and processed for quantification of inflammatory and metabolic gene expression with RT-PCR. All data were analyzed using General Linear Models Procedures in SPSS (IBM) and Tukey Post Hoc test with a $\mathrm{P}<0.05$ considered statistically significant.

Results: AngII dose significantly influenced SBP $(\mathrm{P}=0.003)$, with the $800(\mathrm{SBP}=131 \pm 4 \mathrm{mmHg})$ and 1200 $(131 \pm 4) \mathrm{mmHg}$ doses demonstrating a dramatic increase in as compared to the $400(113 \pm 4)$ and saline control $(110 \pm 5)$. Urinary albumin excretion aligned with the SBP data $(\mathrm{P}=0.003)$, with $1200(696 \pm 450 \mathrm{ug} / \mathrm{mL})$ and 800 $(600 \pm 416)$ having greater 24-hour urinary albumin excretion as compared to mice administered 400 (26 \pm 9$)$ and $0(12 \pm 6)$. Quantification of genes associated with inflammation (Il1b, Il6, Ifng, Tfgb1, Stat3, Cd3e, Ccr2, Cd19, Nfkb1) yielded minimal differences between the AngII doses, while there were strong dose dependent effects on the mRNA expression of key enzymes involved in vascular remodeling (Acta2, $\mathrm{P}<0.001$ ), oxidative stress (Nox4, $\mathrm{P}=0.02$ ), nitric oxide metabolism (Nos1, $\mathrm{P}<0.001$ ), and renal steroid hormone signaling (Hsd11b1, $\mathrm{P}=0.004$ ). Dosage of AngII also significantly $(\mathrm{P}=0.014)$ influenced weight gain over the 4-week study, with AngII dose being inversely associated with weight gain despite correction for caloric intake. Hepatic mRNA expression of Hmgcl (a key ketogenic enzyme) was significantly $(\mathrm{P}=0.007)$ upregulated in the 1200 treatment group that also had the lowest weight gain amongst the different AngII treatments.

Conclusion: Our study confirms the pressor response to increasing doses of chronic AngII administration, with a maximum SBP and albuminuria occurring with as little as $800 \mathrm{ng} / \mathrm{kg}^{\star}$ minute after 4 weeks. The mRNA expression of leukocyte-specific markers and inflammatory mediators in the kidney were not markedly influenced by AngII dose, suggesting other molecular and physiological processes (perhaps oxidative stress) play a greater role in the pressor response to increasing doses of AngII. Higher doses of AngII were associated with less weight gain over the 4-week infusion period, which could be mediated by altered metabolic state (perhaps ketosis) in mice with higher AngII infusion. Additional studies are needed to validate these findings and highlight how AngII alters the structure-function relationships of the kidneys, vasculature, and liver in preclinical animal models of human disease.

\section{References}

1. Coffman TM. The inextricable role of the kidney in hypertension. J Clin Invest. 2014;124(6):2341-2347. doi:72274 [pii].

2. Guzik TJ, Hoch NE, Brown KA, et al. Role of the T cell in the genesis of angiotensin II induced hypertension and vascular dysfunction. J Exp Med. 2007;204(10):2449-2460. doi:jem.20070657 [pii].

3. Trott DW, Thabet SR, Kirabo A, et al. Oligoclonal CD8+ T cells play a critical role in the development of hypertension. Hypertension. 2014;64(5):1108-1115. doi:10.1161/HYPERTENSIONAHA.114.04147 [doi].

4. Lu H, Wu C, Howatt DA, et al. Angiotensinogen Exerts Effects Independent of Angiotensin II. Arterioscler Thromb Vasc Biol. 2016;36(2):256-265. https://doi.org/10.1161/ATVBAHA.115.306740. doi:10.1161/ATVBAHA.115.306740.

5. Kouyama R, Suganami T, Nishida J, et al. Attenuation of dietinduced weight gain and adiposity through increased energy expenditure in mice lacking angiotensin II type 1a receptor. Endocrinology. 2005;146(8):3481-3489. doi:en.2005-0003 [pii].

Financial Disclosures: None reported.

Support: J.C.G is funded by grants from Liberty University College of Osteopathic Medicine, National Institutes of Health (R01DK113632-01A1, Co-investigator), and the American Osteopathic Association (\#19133749, Principal Investigator).

Ethical Approval: All animal experiments were conducted in accordance with policies of the National Institutes of Health (NIH) Guide for the Care and Use of Laboratory Animals and the Liberty University Institutional Animal Care and Use Committee (\#5.170421).

Informed Consent: N/A

Poster No.: *B29

Abstract No.: 69

Category: Basic Science

Research Focus Area: Chronic Diseases \& Conditions

\section{The dual nature of the distal phalanx: the bony cap}

${ }^{1}$ Shannon Elizabeth Smith, OMS-III; ${ }^{2}$ Laurel Yohe; ${ }^{3}$ Nikos Solounias

${ }^{1}$ New York Institute of Technology (NYITCOM); ${ }^{2}$ Department of Earth and Planetary Sciences, Yale University; ${ }^{3}$ Department of Anatomy, New York Institute of Technology (NYITCOM) 
Context: It has been recognized as early as the 1800s that the apex of the distal phalanx has a distinct embryological development from the main shaft of the distal phalanx, but most modern studies do not highlight this. ${ }^{1,2,3,4}$ The distal phalanx shaft is endochondral, while the bony cap is intramembranous. ${ }^{5}$ Our study reiterates and highlights this, and names the apex "the bony cap" to distinguish it. This knowledge can help DOs lead the way in regenerative medicine for our patients and students.

Objective: To identify, name, and revive the existence of the bony cap and postulate its role in human regenerative medicine, our anatomical study reiterates and highlights the special properties of the bony cap to distinguish it. We describe and revive its presence, and we identify it in three mammalian species: humans, cats and horses (Homo sapiens, Felis catus domestica, and Equus caballus). By terming the bony cap, we hope to illuminate its distinct identity and role in limb regeneration.

Methods: First, we conducted a literature review with materials about the bony cap from the 1800s-present day. Next, we used samples of three mammals to conduct our own study of the bony cap: humans, domesticated cat, and domesticated horse (Homo sapiens, Felis catus domestica, Equus caballus).

The bony cap in human embryos was investigated from specimens of the Carnegie Collection of the Walter Reed Army Institute of Research - National Museum of Health and Medicine. The specimens were studied under normal light at 20X and 40XWe also Micro-CTd three cadaveric human adult distal phalanx specimens from NYITCOM's anatomy lab. All histology was done with hematoxylin and eosin stain.

The fifth digit on the left pes of an adult domesticated cat (NS 292) was selected for figures and Micro-CT. We also performed a Micro-CT of the bony cap from a fetal cat (body length $30 \mathrm{~mm}$; NS 294. purchased from Ward's Biological Supply.

We used three samples of three horse fetuses. The whole-body length in millimeters was measured with digital calipers from frontal bone to rump. A small horse was Micro-CTd (NS 292; $210 \mathrm{~mm}$ whole body length). A larger near-full term horse was sectioned with a saw. The pes (NS 293; $762 \mathrm{~mm}$ whole body length) was stained with iodine and Micro-CTAnother near-full term horse (NS 801; $750 \mathrm{~mm}$ whole body length) was sectioned longitudinally. The other manus of the same specimen was sectioned transversely near the proximal, middle and distal end. The horse specimens were purchased from Ward's Biological Supply.

The NYITCOM Micro-CT had the following parameters: voltage: 45 , current: 177 , resolution: $2 \mathrm{~K}$, filter: $0.002 \mathrm{~mm}$
Copper exposure: 1850 , spot size: 6.4 Micro-CT ns, rotation steps: 0.3, frames: 7, random movement: 20, 360-degree scan. The Yale Micro-CT was used for the horse and the adult cat. We used a $0.1 \mathrm{~mm}$ copper filter.

Results: The Human Fetus and Adult:

The bony cap can be distinguished from the endochondral distal phalanx as early as 14 weeks of development. The bony cap can be observed in longitudinal sections of the digit. $^{7}$

In our data, fingers show representative typical bony caps that are best observed in a $22 \mathrm{~mm}$ embryo (stage 22). The apical cap is enlarged on the distal ventral side as it is in the adult.

In the adult skeleton, the bony cap is demarcated by the rough apex. Interosseous ligaments connect the middle phalanx to the bony cap of the distal phalanx. These ligaments create two tunnels between the ligaments themselves and the distal phalanx. The arteries pass through these tunnels, contributing to the large vascular bundle that supplies the nail bed and passes ventrally.

The Fetal and Adult Domestic Cat

The Micro-CT imaging of the $30 \mathrm{~mm}$ cat embryo displays ossified bony caps on all developing digits. The bony cap has an elongated crescent shape with a distinct pointed distal end and a hollow center. It is situated ventral to the keratinous claw. and it is hollow. This is because the distal phalanx does not insert into it as it does in other species.

The Fetal and Adult Horse

The Micro-CT imaging of specimen NS 294 shows the distalmost part of the bony cap as having five tubes lined up next to each other. ${ }^{8}$ These figures distinguish that the bony cap at this stage is entirely independent of the distal phalanx proper.

The mid-sagittal section of front left hoof of a near full term fetal horse (762 mm) (NS 293) displays a white line that outlines the distal phalanx where the keratin comprising the hoof originates. We suspect that this white outline is the bony cap.

The distal phalanx of the adult horse has two types of surfaces: a rough one that binds to the keratinous hoof and a smooth one proximal to the rough that is free of keratin covers. We postulate that the rough portion is the bony cap. Four foramina are visible on the proximal side.

Conclusion: The bony cap is a distinct unit of intramembranous origin found at the apex of the distal phalanx. It has an inductive role in bone regeneration and keratin development. It has been described in the literature; however, its embryological and anatomical significance has been overlooked. The ossification of the bony cap precedes that of the endochondral part of the distal phalanx. The bony cap as an intramembranous structure is an 
independent entity from the endochondral distal phalanx during early development. The two will fuse into a single bone in adulthood. This is a similar pattern to the development of the clavicle, innominates, and scapula. In adulthood, it appears as a thin, rough layer of bone surrounding the tip of the cartilaginous distal phalanx.

In all three species, the bony cap is a thin veneer. In humans and horses, it covers and extends to the middle of the distal phalanx. In the cat, the bony cap lies adjacent to the distal phalanx but not cover the distal phalanx due to the specialization of the claw(8). In humans, cats, and horses it is associated with keratin and acts as the interface between the distal phalanx and the keratinized surface of the nail, claw, or hoof. ${ }^{9}$ This interface occurs at the apex of the distal phalanx no matter the number of the phalanges.

Bone regeneration has been studied in higher vertebrates and it has been reported that the distal-most aspect of the distal phalanx has an inductive role in bone regrowth. ${ }^{5}$ In other literature, it has been said that the nail organ is responsible for bone growth. ${ }^{10}$ The location of the bony cap correlates with the findings in these studies and could be the organ responsible for bone growth. This could be the key discovery in understanding how to apply regenerative medicine in human limb regrowth.

The recognition of the bony cap with a unique name and its existence in three species reinvigorates its identity and illuminates a promising link with regenerative biology. ${ }^{5,6,10}$

\section{References}

1. Dixey FA. II. On the ossification of the terminal phalanges of the digits. Proceedings of the Royal Society of London. 1881;31(206211):63-71. doi:10.1098/rspl.1880.0009

2. Ewart JC. The development of the skeleton of the limbs of the horse PT I-III. Journal of Comparative Pathology and Therapeutics. 1894;7:236-369. doi:10.1016/s0368-1742(9480002-5)

3. Ewart JC. The Second and Fourth Digits in the Horse: their Development and Subsequent Degeneration. Proceedings of the Royal Society of Edinburgh. 1895;20:185-191. doi:10.1017/ s0370164600048525

4. Mettam AE. The Os Pedis in Ungulates. Nature. 1894;49(1267):341341. doi:10.1038/049341a0

5. Sensiate LA, Marques-Souza H. Bone growth as the main determinant of mouse digit tip regeneration after amputation. Scientific Reports. 2019;9(1). doi:10.1038/s41598-019-45521-4

6. Zhao W, Neufeld DA. Bone regrowth in young mice stimulated by nail organ. Journal of Experimental Zoology. 1995;271(2):155-159. doi:10.1002/jez.1402710212

7. Hamrick MW. Development and evolution of the mammalian limb: adaptive diversification of nails, hooves, and claws. Evolution and Development. 2001;3(5):355-363. doi:10.1046/j.1525-142x. 2001.01032.x
8. Homberger DG, Ham K, Ogunbakin T, et al. The structure of the cornified claw sheath in the domesticated cat (Felis catus): implications for the claw-shedding mechanism and the evolution of cornified digital end organs. Journal of Anatomy. 2009; 214(4):620-643. doi:10.1111/j.1469-7580.2009.01068.x

9. Kamibayashi Y, Abe S, Fujita T, Imai A, Komatsu K, Yamamoto Y. Congenital ectopic nail with bone deformity. British Journal of Plastic Surgery. 1998;51(4):321-323. doi:10.1054/bjps.1997. 0138

10. Zhao W, Neufeld DA. Bone regrowth in young mice stimulated by nail organ. Journal of Experimental Zoology. 1995;271(2):155-159. doi:10.1002/jez.1402710212

Financial Disclosures: None reported.

Support: None reported.

Ethical Approval: Exempt

Informed Consent: N/A

Poster No.: B30

Abstract No.: 72

Category: Basic Science

Research Focus Area: Chronic Diseases \& Conditions

Therapeutic Effects of Lymphatic

Pump Treatment on

Lymphangiogenesis and Inflammatory Cytokines in Lymph Nodes of Rats with AdjuvantInduced Arthritis

${ }^{1}$ Kelly Margaret Blucher, MBS, OMS-I; ${ }^{2}$ Shreya Jain; ${ }^{2}$ Daniel Weber; ${ }^{2}$ Calley Gober; ${ }^{3}$ Brian Zanotti; ${ }^{3}$ Ryan Incrocci, MS; ${ }^{3}$ Rosalinda Del Toro, MS; ${ }^{3}$ Michelle Swanson-Mungerson, $\mathrm{PhD} ;{ }^{3}$ Michael V. Volin, PhD

${ }^{1}$ Midwestern University Chicago College of Osteopathic Medicine (MWU-CCOM); ${ }^{2}$ Midwestern University Chicago College of Osteopathic Medicine (MWU-CCOM; ${ }^{3}$ College of Graduate Studies, Midwestern University Chicago College of Osteopathic Medicine (MWU-CCOM)

Context: Rheumatoid arthritis (RA) is a chronic inflammatory disease caused by self-reactive lymphocytes, eventually destroying joints. To study this disease and potential treatments, the adjuvant-induced arthritis (AIA) rat model can be used. In RA, there is a collapse of appropriate lymphatic drainage $e^{1,2}$ and an increase in inflammatory cytokines in the lymph nodes. ${ }^{3}$ The osteopathic treatment, Lymphatic pump treatment (LPT), has the potential to increase lymphatic circulation and decrease inflammation. ${ }^{4}$ 
Objective: For this study, we tested the hypothesis that LPT would prevent the collapse of lymphatic drainage and promote lymphatic vessel growth in a rat model of arthritis. Furthermore, we hypothesized that this increased lymphatic movement from LPT would allow for the systemic distribution of pro-inflammatory cytokines to alleviate local inflammation at the inflamed joint.

Methods: Experimental rats were immunized with Complete Freund's adjuvant on day zero. Control rats for this study were non-immunized and non-arthritic. After the onset of adjuvant-induced arthritis, rats received either LPT or sham treatment three times a day for six days. On day 21, the draining popliteal lymph nodes (PLN) were harvested and analyzed for cytokine levels (IL-1ß, IL-4, IL-6, IL-17, IFN- - , TNF- $\alpha$, VEGF-C) or VEGFR-3 by quantitative RT-PCR. Additionally, immunohistochemistry (IHC) was used to visualize VEGF-C and VEGFR-3 expression in the PLNs. The experiment used thirty-six rats total and all experiments were statistically analyzed initially by a oneway analysis of variance followed by a Tukey post-hoc test to determine if significant differences were identified among the groups.

Results: Injected rats successfully developed arthritis as shown through a statistically significant increase in ankle circumference and articular index score (AIS). The LPT group showed a slight decrease in ankle circumference and AIS. No statistical differences were seen among the cytokines analyzed in the draining PLNs when comparing the LPT and sham-treated groups. IHC showed an increase in VEGF-C and VEGFR-3 expression in injected animals compared to non-injected animals, with the PLNs from the LPT group having the highest expression area of VEGF-C and the lowest expression area of VEGFR-3 within the injected treatment groups.

Conclusion: The injected LPT group showed a slight reduction in ankle circumference and AIS, suggesting that LPT helped alleviate edema and inflammation. While the cytokines analyzed showed no difference between arthritic treatment groups, it is possible that other cytokines may have been impacted, which can be analyzed in future studies. Additionally, in the PLN, LPT trended towards elevated levels of VEGF-C, an important lymphatic vessel growth factor. Taken together, the data suggest LPT may alleviate inflammation in rats through the elevation of a lymphatic growth factor. Through these results, we have better insight into the effectiveness of LPT in treating chronic inflammatory diseases such as RA with the hopes of applying these techniques to improve patient care.

\section{References}

1. Foltz V et al. Power Doppler ultrasound, but not low-field magnetic resonance imaging, predicts relapse and radiographic disease progression in rheumatoid arthritis patients with low levels of disease activity. Arthritis and rheumatism 2012;64:67-76. PMID: 21904998 doi:10.1002/art.33312

2. Benaglio $\mathrm{F}$ et al. The draining lymph node in rheumatoid arthritis: current concepts and research perspectives. Biomed Res Int. 2015, 420251. PMID: 25793195 doi:10.1155/2015/420251.

3. Smith MD, et al. Successful treatment of rheumatoid arthritis is associated with a reduction in synovial membrane cytokines and cell adhesion molecule expression. Rheumatology (Oxford). 2001;40(9):965-977. PMID: 11561106 doi:10.1093/rheumatology/ 40.9.965

4. Schander A, Downey HF, Hodge LM. Lymphatic pump manipulation mobilizes inflammatory mediators into lymphatic circulation. Exp Biol Med (Maywood). 2012;237(1):58-63. PMID: 22169162 doi: 10. 1258/ebm.2011.011220

Financial Disclosures: This work was funded by the Biomedical Sciences Program at Midwestern University.

Support: The current study was funded by the Biomedical Sciences Program at Midwestern University granted to Dr. Swanson-Mungerson.

Ethical Approval: IACUC approved - IACUC 1935

Informed Consent: N/A (Animal Study Only)

${ }^{\star}$ Poster No.: ${ }^{\star B} 31$

Abstract No.: 73

Category: Basic Science

Research Focus Area: Chronic Diseases \& Conditions

Evaluation of the effects of Marfan pathogenesis and losartan treatment on aorta, coronary, and cerebral arteries in a Marfan Syndrome mouse model

${ }^{1}$ Bailey Kuechenmeister, OMS-III; ${ }^{2}$ Brikena Hoxha; ${ }^{2}$ Tala Curry; ${ }^{1}$ T. Bucky Jones, PhD; ${ }^{3}$ Mitra Esfandiarei, PhD

${ }^{1}$ Midwestern University Arizona College of Osteopathic Medicine (MWU/AZCOM)

${ }^{2}$ College Of Graduate Studies, Midwestern University Arizona College of Osteopathic Medicine (MWU/AZCOM)

Context: Marfan syndrome is a connective tissue disorder caused by mutations in the fibrillin-1 gene. ${ }^{1,2}$ This mutation results in a variety of phenotypic changes in the musculoskeletal, cardiovascular, and pulmonary systems, with a 
notable vascular effect leading to aortic aneurysm, dissection, and rupture ${ }^{2,3}$ increasing morbidity and mortality. Prior studies have shown the protective effect Losartan on the aorta ${ }^{4}$ however there is limited investigation surrounding its effect on smaller vessels.

Objective: Prior studies have demonstrated the protective effects of Losartan, an angiotensin II type I receptor (AT1R) blocker, blocker, on slowing the progression of aortic root aneurysm in the well-established mouse model of Marfan Syndrome (Fbn1 +/- p. Cys1041Gly)4. In this study, we are further investigating the impact of Marfan Syndrome (MFS) pathogenesis and Losartan treatment on smaller vessels such as the posterior cerebral artery and coronary arteries. Methods: Mice (male + female) were divided into experimental groups based on genotype and treatment plan: Control, MFS, MFS + 0.6g/L losartan. Drug therapy consisted of $0.6 \mathrm{~g} / \mathrm{L}$ of losartan in drinking water that was replenished three times a week. Water intakes were recorded to ensure the consistency of treatment across experimental groups. At 7 months of age, in vivo ultrasound imaging was performed to measure aortic root diameters, aortic pulse wave velocity (PWV), and peak blood flow of the coronary and posterior cerebral arteries5,6,7. In order to assess medial elastin fragmentation in the aortic wall, $5 \mu \mathrm{M}$ aortic cross-sections were subjected to Verhoeff-Van Gieson (VVG) staining. Blood pressure (BP) measurements were also obtained, using the tail-cuff method. All image analyses were conducted by a single observer blinded to animal genotypes. Comparisons of parameters among treatment groups were made using one-way analysis of variance (one-way ANOVA) followed by Tukey's multiple-comparison test. Comparisons of parameters between two groups were made by two-tailed Student's t-test, where a $p$ value of $p<0.05$ is considered to be statistically significant. While this study does not directly involve OMT, delaying the progression of vascular dysfunction and aortic aneurysm can improve the quality of life for these patients, providing a much-needed timeframe for other interventions, and allowing for a larger variety of osteopathic manipulations to reduce chronic pain in this vulnerable patient population.

Results: The aortic root diameter at the sinus of valsava was increased in MFS mice $(2.040 \pm 0.044, \mathrm{P}=<0.0001)$ in comparison to CTRL mice (1.644 \pm 0.032$)$ and MFS mice treated with Losartan demonstrated a significant decrease in the aortic root diameter $(1.611 \pm 0.017, \mathrm{P}=<0.0001)$. The pulse wave velocity was increased in MFS mice (5.805 $\pm 0.775, \mathrm{P}=<0.0001)$ when compared to CTRL $(1.023 \pm 0.0464)$ and treatment with Losartan in MFS mice significantly decreased the pulse wave velocity (1.009 $\pm 0.0284, \mathrm{P}=<0.0001)$ indicating improvement in aortic stiffness. The number of aortic elastin fragments was increased in MFS mice $(66.50 \pm 4.97)$ vs. CTRL mice $(41.12 \pm 3.82, \mathrm{P}=0.0015)$ and Losartan significantly reduced elastin fragmentation in MFS mice $(32.58 \pm 1.09)$ as compared to non-treated MFS $(66.50 \pm 4.97, \mathrm{P}=<0.0001)$. Elastin fragment length was decreased in MFS mice $(127.3 \pm 12.16)$ vs. control mice $(243.3 \pm 13.59, \mathrm{P}=0.0011)$ and Losartan significantly increased elastin fragment length in MFS mice (413.4 \pm 16.42$)$ as compared to non-treated MFS mice $(127.3 \pm 12.16, P=<0.0001)$. These findings of decreased number of elastin fragments and increased elastin fragment length, demonstrate improvement in aortic wall structural integrity with Losartan treatment. The systolic coronary peak flow demonstrates a statistically significant decrease in MFS mice $(146.8 \pm 22.31)$ when compared to CTRL ( $304.5 \pm 37.74, \mathrm{P}=0.005)$, however we do not see a statistically significant difference in MFS mice treated with Losartan $(96.67 \pm 12.61, \mathrm{P}=0.41)$. There is no statistical significance in diastolic coronary peak flow between CTRL vs. MFS and MFS vs. MFS + Losartan. There is no statistical significance in comparison of the posterior cerebral artery peak flow between CTRL to MFS and MFS to MFS + Losartan. There is also no statistical significance in the systolic and diastolic BP between the groups.

Conclusion: Our results show that Losartan has beneficial effects in delaying the progression of aortic aneurysm, reduces vessel wall stiffness, and reduces elastin fragmentation in the aortic root. These findings may allow for improvement in the quality of life for Marfan syndrome patients and also open up possibilities for interventions using OMT to treat the musculoskeletal phenotypic changes and chronic pain seen in this patient population. This study also provides an early insight into the disease progression of MFS in the less investigated coronary and cerebral arteries, as well as investigates the preliminary potential effects of Losartan on peak flow in coronary and cerebral vessels in the well-established MFS mouse model.

\section{References}

1. Dietz HC, Cutting GR, Pyeritz RE, et al. Marfan syndrome caused by a recurrent de novo missense mutation in the fibrillin gene. Nature. 1991;352(6333):337-339. doi: 10.1038/352337a0.

2. Judge DP, Dietz HC. Marfan's syndrome. Lancet. 2005;366(9501): 1965-1976. https://doi.org/10.1016/S0140-6736(05)66995-4

3. Pyeritz RE. The Marfan syndrome. Annu Rev Med. 2000;51:481510. DOI: 10.1146/annurev.med.51.1.481

4. Yang HH, Kim JM, Chum E, van Breemen C, Chung AW. Effectiveness of combination of losartan potassium and doxycycline versus single-drug treatments in the secondary prevention of thoracic aortic aneurysm in Marfan syndrome. J Thorac Cardiovasc Surg. 2010;140(2):305-312 e302. doi: 10.1016/j.jtcvs.2009.10.039 
5. Gao S, Ho D, Vatner DE, Vatner SF. Echocardiography in Mice. Curr Protoc Mouse Biol. 2011;1:71-83. doi: 10.1002/9780470942390. mo100130

6. Esfandiarei M, Hoxha B, Talley NA, et al. Beneficial effects of resveratrol and exercise training on cardiac and aortic function and structure in the 3xTg mouse model of Alzheimer's disease. Drug Des Devel Ther. 2019;13:1197-1211. doi: 10.2147/DDDT. S196119

7. Cui JZ, Lee L, Sheng X, et al. In vivo characterization of doxycyclinemediated protection of aortic function and structure in a mouse model of Marfan syndrome-associated aortic aneurysm. Sci Rep. 2019;9(1):2071. doi: 10.1038/s41598-018-38235-6

Financial Disclosures: None reported.

Support: This study received funding from the National Institutes of Health.

Ethical Approval: This study was reviewed and approved by IACUC, \#2853.

Informed Consent: N/A

Poster No.: ${ }^{\star B} 32$

Abstract No.: 76

Category: Basic Science

Research Focus Area: Chronic Diseases \& Conditions

Novel Approach to Organ Transport with OxyVita, A Next Generation Hemoglobin- based Oxygen Carrier in Modified Preservation Solution and Storage Box

${ }^{1}$ Chirag Soni, OMS-II; ${ }^{1}$ Jonah Duran, MS; ${ }^{1}$ Komail Jafri, BS; ${ }^{1}$ Sachin Shah, BME; ${ }^{2}$ Michael Mazzariello, II; ${ }^{1}$ Alexsandra Taylor, MS; ${ }^{1}$ Andrew Bell, BS; ${ }^{1}$ Karan Kumar, MS; ${ }^{1}$ Shannon Kiss, BS; ${ }^{3}$ Brian Wollocko, BA; ${ }^{4}$ Nilank Shah, MD, MS, CFN, CNC; ${ }^{5}$ Hanna Wollocko, MD

${ }^{1}$ Touro College of Osteopathic Medicine-Middletown (TouroCOM); ${ }^{2}$ Georgetown University

${ }^{3}$ Renaissance School of Medicine at Stony Brook University; ${ }^{4}$ Department of Medical; Physiology/Pharmacology, Touro College of Osteopathic Medicine-Middletown (TouroCOM)

${ }^{5}$ Department of Internal Medicine, Touro College of Osteopathic Medicine-Middletown (TouroCOM)

Context: Time is the biggest threat to a successful organ transplantation. Current storage and transport methods reduce the likelihood of post-transplant function, as organs are susceptible to cellular edema, ischemic damage, and oxidative damage. Recent preservation solutions attempt to prevent this organ damage but fail to support metabolic processes and oxygen delivery. ${ }^{2}$ Novel preservation solutions must deliver oxygen effectively to mitigate these harmful shortcomings.

Objective: To improve organ preservation by maximizing oxygen delivery via OxyVita's hemoglobin-based oxygen carrier to support aerobic metabolism. We are focused on optimizing the parameters of the organ preservation solution by adding sucrose and trehalose along with various loading methods for these sugars and examining their interactions with hemoglobin. Ultimately, this solution will be used with the OxyVita Organ Storage Device: a perfusion device that supports the organ with pulsatile flow.

Methods: The hemoglobin concentration of the initial samples of teramer or polymer was measured at absorbances of $538 \mathrm{~nm}$ and $680 \mathrm{~nm}$ using a Hewlett - Packard Agilent 8453 Spectrophotometer and then analyzed with ChemStation software. Based on the initial $\mathrm{Hb}$ concentration, samples were then diluted using phosphate buffer and Ringers solution to achieve both $1.5 \%$ and $3.0 \% \mathrm{Hb}$ concentration solutions. All $1.5 \% \mathrm{Hb}$ and $3.0 \% \mathrm{Hb}$ solutions were prepared to have a total volume of $10 \mathrm{ml}$, and then designated to either the Loading Protocol or the NonLoading Protocol for the uptake of the sugars. In order to test whether the order of addition of the sugars affected oxygen saturation, two sets of samples were produced. The first set involved adding the sugar to the tetramer or polymer, followed by the buffer. The second set involved adding the sugar to the buffer, followed by the tetramer or polymer according to the Loading protocol. The Loading Protocol entailed placing the solutions on hot plate stirrers in a water bath at the constant temperature of $37^{\circ} \mathrm{C}$ while being stirred using stirring rods. For this method, half the sugar was added to the solution at time 0 , and the other half was introduced into the solution at time 30 minutes. The solutions were removed from the hot plate stirrers after 60 minutes. The Non-Loading Protocol consisted of placing the solutions on The Belly Dancer Shakers for 5 minutes after the addition of the sugars. Once solutions completed their loading/non-loading method, solutions were analyzed using the Spectrophotometer protocol mentioned above. Ratios to analyze oxygen saturation for hemoglobin were measured at absorbances of 576 and $542 \mathrm{~nm}$.

Results: The initial results show the sample sets that followed the loading protocol had significant increases in their hemoglobin oxygen saturation in comparison with the samples that followed the non-loading protocol. Oxygen saturation of hemoglobin reached as high as a 3.7\% increase for the solutions that followed the loading protocol. These results suggest that increased interaction time between the sugar and the hemoglobin during the loading method lead to an increased oxygen saturation of 
hemoglobin. Evidence of this can also be observed since the solutions that added the sugars after the hemoglobin and buffer solution were mixed generated hemoglobin with increased oxygen saturation compared to those solutions in which the polymer/tetramer were added after the completion of the loading method and sugar addition. Regarding the concentrations of the sugars, the $5.2 \mathrm{mg} / \mathrm{ml}$ of trehalose solution produced an average of a $14.23 \%$ increase in the $1.5 \%$ hemoglobin solution and an average of a $7.67 \%$ increase in the $3.0 \%$ hemoglobin solution in comparison to the $.52 \mathrm{mg} / \mathrm{ml}$ trehalose solutions. It was also observed that $12.75 \mathrm{mg} / \mathrm{ml}$ of sucrose solution produced the greatest oxygen saturation in comparison to solutions produced with various decrements of sucrose concentrations. These results indicate that the optimal preservation solution contains $12.75 \mathrm{mg} / \mathrm{ml}$ sucrose, and $5.2 \mathrm{mg} / \mathrm{ml}$ trehalose.

Conclusion: Our current data suggests that the optimal preservation solution with $12.75 \mathrm{mg} / \mathrm{ml}$ sucrose and $5.2 \mathrm{mg} /$ $\mathrm{ml}$ trehalose would provide organs with the most efficient delivery of oxygen. These sugars would be added to the diluted tetramer/polymer while using a loading process where the sugar is added in half quantities at time zero and time 30 minutes. According to our results, the indicated protocol for the preservation solution would optimize the oxygen delivery of OxyVitaHb for its use in the Organ Storage Box. Future work will aim towards improving the functions of the Organ Storage Box, which is a critical component of this research project, including controls for measuring flow rate and oxygen delivery to maintain maximized oxygen carrying capacity of the preservation solution. Another area of future research includes analysis of organ tissue after storage in order to determine areas of tissue injury and organs' viability prior to transplantation. Only through delivery of the oxygen into microcirculation via the organ storage solution and perfusing the organ via the Organ Storage Box, metabolism can be maintained and stable organ conditions can be achieved, leading to a higher possibility of survival of the transplanted organ's tissue.

\section{References}

1. Guibert EE, Petrenko AY, Balaban CL, Somov AY, Rodriguez JV, Fuller BJ. Organ Preservation: Current Concepts and New Strategies for the Next Decade. Transfus Med Hemother. 2011; 38(2):125142. doi:10.1159/000327033

2. Barker CF, Markmann JF. Historical overview of transplantation. Cold Spring Harb Perspect Med. 2013; 3(4):1-18. doi: 10.1101/ cshperspect.a014977
Financial Disclosures: None reported.

Support: None reported.

Ethical Approval: None required.

Informed Consent: Not relevant.

Poster No.: *B33

Abstract No.: 79

Category: Basic Science

Research Focus Area: Osteopathic Philosophy

\section{What learning resources helped you succeed in medical school?}

${ }^{1}$ Donato Mignone, OMS-IV; ${ }^{2}$ Keya Shah, DO; ${ }^{3}$ Leslie Goldstein, PharmD

${ }^{1}$ New York Institute of Technology (NYITCOM); ${ }^{2}$ Department of Medicine, New York University Langone Long Island Hospital; ${ }^{3}$ Department of Clinical Specialties, New York Institute of Technology (NYITCOM)

Context: As medical schools around the country are moving towards a student-centered curriculum, students must have access to information about resources that have had high success rates. ${ }^{2}$ Medical educators can utilize this data to provide high-quality resources to students to improve medical education. Also, Understanding the reasons for student choices can give insight into how medical students are influenced and could eventually help guide administrators. $^{3}$

Objective: To determine what medical education learning resources correlate to academic success during preclinical medical school years. Secondary objectives sought which factors influence students to select specific resources.

Methods: A voluntary, confidential survey was employed to gather information on various learning resources and how their use correlates with a student's academic performance. The participants recorded how often they used a specific learning resource, reasons behind their selections, and board score ranges. They also recorded whether they preferred longer, comprehensive lectures or shorter, focused lectures. The survey was distributed via email to third and fourth-year medical students at the NYITCOM campuses in Old Westbury and Arkansas and recorded 124 responses.

The median was used to calculate central tendencies of the amount each resource was used and the reasons for selecting resources because they were collected as ordinal data. The correlation of resource usage to exam scores was calculated with a Spearman's Rho test also because we collected ordinal data. 
These results are significant to the osteopathic profession because they may be used to enhance osteopathic medical education. Students can benefit by having more informed choices in selecting their medical education resources and medical educators can benefit by offering strong resources to their students.

Results: The most commonly used resources were lecture PowerPoints, First Aid, Anki, and visual mnemonics, all with a median use of "more than once a week". Comprehensive textbooks were used with a median of "less than once a week". The most important reasons for using a resource were: easy to understand, convenient, reliable, fitting my learning style, and "high yield", with a median of "very important". The least important reason was to be recommended by faculty with a median of "slightly important”. United States Medical Licensing Examination (USMLE) Step 1 score was positively correlated with using Anki every (Kruskal-Wallis 10.123, $\mathrm{p}<.05$ ), and negatively correlated with lecture powerpoints $(-.327, \mathrm{p}<.01)$. We found no significant correlates with the Comprehensive Osteopathic Medical Licensing Examination (COMLEX) Level 1 or COMLEX Level 2.

Conclusion: Students found faculty resource recommendations to be "slightly important." Some students commented that they were more tailored to faculty lectures than board exams. This result agrees with previous data that recommendations of faculty are deemed less important for resource selection. If educators want to encourage the use of certain resources, it may be more beneficial to increase access to certain successful resources, rather than simply recommending them.

Using Anki everyday correlated to higher USMLE Step 1 scores. Anki uses spaced interval training strategies that are well supported in educational literature.4 Additionally, more frequent use of lecture PowerPoints correlated with lower USMLE Step 1 scores. Future studies are required to explain whether this correlation is due to the style of learning or differences in the material covered compared with material tested on the board exams.

Common to many survey studies, some inherent limitations include dishonest answers, unanswered questions, and differences in understanding and interpretation of survey questions. Also, for the simplicity of the survey, we chose to ask about the frequency of resource usage, which may not reflect the actual amount of time that students spent using them. There was a lack of significant data on the less used resources, so greater participation may be required for these resources. Also, because the study was voluntary, the sample of students may not be representative of the entire student population. While this study aims to assess academic success by using board scores, overall success as a future physician is not solely determined by board scores, but rather a wide variety of factors. It is hoped that research like this can offer students and administrators insight into the effectiveness of various medical education resources.

\section{References}

1. O'Hanlon R, Laynor G. Responding to a new generation of proprietary study resources in medical education. J Med Libr Assoc. 2019;107(2):251-257. doi:10.5195/jmla.2019.619

2. Judd T, Elliott K. Selection and Use of Online Learning Resources by First-Year Medical Students: Cross-Sectional Study. JMIR Med Educ. 2017;3(2):e17. Published 2017 Oct 2. doi:10.2196/mededu. 7382

3. Al-Hazmi A. Educational resources used by medical students in primary healthcare rotation: A cross sectional study. Pak J Med Sci. 2016;32(2):361-364. doi:10.12669/pjms.322.9784

4. Deng F, Gluckstein JA, Larsen DP. Student-directed retrieval practice is a predictor of medical licensing examination performance [published correction appears in Perspect Med Educ. 2016 Nov 18;:]. Perspect Med Educ. 2015;4(6):308-313. doi:10.1007/ s40037-015-0220-x

Financial Disclosures: None reported.

Support: None reported.

Ethical Approval: This research has been approved for exemption by the NYITCOM IRB, BHS-1574.

Informed Consent: Potential subjects were given an email that requested their voluntary participation and assured that the survey was voluntary, anonymous, confidential and was sent at the request of the investigators only, and not the NYITCOM administration. The email also shared the generic REDCap confidentiality statement.

Poster No.: *B34

Abstract No.: 80

Category: Basic Science

Research Focus Area: Chronic Diseases \& Conditions

A comparison of the effect of EBV LMP2A protein on BTK and STAT3 Phosphorylation between Murine $B$ cell tumors and human Burkitt's lymphoma cells

${ }^{1}$ Mehwish Khan, OMS-IV; ${ }^{2}$ Rosalinda Del Toro, MS; ${ }^{2}$ Ryan Incrocci, MS; ${ }^{2}$ Michelle Swanson-Mungerson, $\mathrm{PhD}$ 
${ }^{1}$ Midwestern University Chicago College of Osteopathic Medicine (MWU-CCOM); ${ }^{2}$ Department of Microbiology and Immunology, Midwestern University Chicago College of Osteopathic Medicine (MWU-CCOM)

Context: After initial infection, Epstein-Barr virus (EBV) transitions into a latent state in B lymphocytes. Our lab investigates the effect of Latent Membrane Protein 2A (LMP2A), an EBV encoded transmembrane protein, on signaling in B cells, especially B cells containing the 8;14 chromosomal translocation seen in Burkitt's Lymphoma. This pro-survival translocation causes overexpression of Myc, a gene that promotes tumor progression.

Objective: In order to ascertain if blocking LMP2Adependent signaling would impact tumor survival and development in vivo, we initiated experiments that crossed transgenic mice to generate mice that overexpress both LMP2A and MYC in B lymphocytes. We hypothesized that the effects of LMP2A on survival that we saw in human B cell Burkitt's lymphoma cells would be paralleled in a transgenic mice model.

Methods: We crossed LMP2A transgenic (-Tg) mice with $\lambda$-MYC-Tg mice that contain the human MYC gene behind the lambda promoter, so that MYC will be constitutively expressed in B cells. ${ }^{2}$ After assessing mice for tumor formation, the mice from each genetic strain were euthanized, and their tumors were collected. We obtained protein isolates from tumors and assessed phosphorylated BTK as well as phosphorylated STAT3 levels by Western blot analysis.

Results: Kaplan-Meijer curves indicated that LMP2A/ $\lambda$-MYC transgenic mice demonstrated an accelerated onset in tumor development, as demonstrated previously. ${ }^{2}$ In contrast to our findings in human Burkitt's lymphoma $B$ cells, the tumor cells from LMP2A/ $\lambda$-MYC transgenic mice did not demonstrate an increase in phosphorylation in either BTK or STAT3 by Western blot analysis.

Conclusion: In the LMP2A/ $\lambda$-MYC tumor model, LMP2A does not activate BTK and/or STAT3, suggesting that the LMP2A/ $\lambda$-MYC tumor model does not recapitulate the effects of LMP2A seen in human B cells.

\section{References}

1. Ryan Incrocci, Levi Barse, Amanda Stone, et al. Epstein-Barr Virus Latent Membrane Protein 2A (LMP2A) enhances IL-10 production through the activation of Bruton's tyrosine kinase and STAT3. Virology. 2017;500:96-102. PMID: 27792904 DOI: 10.1016/j.virol. 2016.10.015

2. Bultema, R., Longnecker R, and M.A. Swanson-Mungerson. Epstein Barr Virus Latent Membrane Protein 2A (LMP2A) accelerates Myc-
Induced Lymphomagenesis. Oncogene. 2009;19;28(11):1471-6. PMID: 19182823 DOI: 10.1038/onc.2008.492

Financial Disclosures: None reported.

Support: None reported.

Ethical Approval: Study was deemed exempt from IRB or IACUC review.

Informed Consent: N/A

Poster No.: *B35

Abstract No.: 88

Category: Basic Science

Research Focus Area: Osteopathic Philosophy

The Importance of Dermatology Education During Osteopathic Preclerkship Curriculum

${ }^{1}$ Vanessa Tan, OMS-II; ${ }^{1}$ Casey Schukow, OMS-IV; ${ }^{1}$ Vito Vitale, OMS-III; ${ }^{2}$ Carolina Restini, PharmD, PhD Assistant Professor

${ }^{1}$ Michigan State University College of Osteopathic Medicine (MSUCOM); ${ }^{2}$ Department of Pharmacology and Toxicology, Midwestern University Chicago College of Osteopathic Medicine (MWU-CCOM)

Context: Osteopathic physicians should be educated on the differences between non-emergent and emergent dermatological conditions so they can continue to provide efficient, quality patient care, regardless of their specialty. Only $40 \%$ of Osteopathic medical schools have a sole dermatology course offered during their pre-clerkship education. This is the first study to investigate the importance of dermatology education in the Osteopathic pre-clerkship curriculum from medical students' perspectives.

Objective: We hypothesize that student's perception of the importance of dermatology education would highlight the need for a single dermatology course during the Osteopathic pre-clerkship curriculum to develop well-rounded Osteopathic physicians. ${ }^{1}$ This study's specific aim and overall purpose are to identify the importance of dermatology education relative to other systems courses in the Osteopathic pre-clerkship curriculum from medical students' perspectives.

Methods: A cross-sectional study was conducted at Michigan State University College of Osteopathic Medicine (MSUCOM) during the months of June and July of 2021. Medical students from every class (2022-2025) were emailed an invitation to volunteer to participate in the study. Google Forms was utilized as a platform to retrieve answers from participants. The study was completely voluntary and 
included an anonymous survey of 19 multiple-choice questions. Participants were made aware that their responses were anonymous and had no impact on their academic performance. MSU IRB approval: STUDY00006234. No personal identifying information was collected, and participation was strictly voluntary. Data was collected from the survey via a 5-point question scale (e.g., "1" resembling lower/lowest importance while " 5 " resembles greater/greatest importance). The results were analyzed via statistical tests (Pearson's Chi-square or Fisher's exact test). These tests were performed with GraphPad Prism 5.0 (GraphPad Software, San Diego, CA). This study presents negligible risks to the participants. As the human body is a functional unit, the absence of in-depth dermatological understanding can pose consequences for future Osteopathic physicians when treating patients, potentially resulting in physical, mental, and spiritual harm. ${ }^{2}$ Only $40 \%$ of Osteopathic medical schools have a sole dermatology course during pre-clerkship education. ${ }^{3}$ Dermatology education is extremely limited in both pre-clerkship and clerkship training, with only a small percentage of institutions offering required dermatology courses and rotations. ${ }^{4}$ This poses a concerning lack regarding dermatology education, as the integumentary system is the largest organ of the body and can suggest underlying medical conditions of other organ systems. ${ }^{1,5}$

Results: A sample of 191 MSUCOM students ( $\mathrm{n}=191)$ have completed the survey. 63 (33\%) were first-years (OMS-I) of the Class of 2025, $41(21.5 \%)$ were OMS-II of the Class of 2024, 44 (23\%) were OMS-III of the Class of 2023, and 43 (22.5\%) were OMS-IV of the Class of 2022. Compared to other pre-clerkship course subjects such as neurology, endocrinology, and gastroenterology, 104 (54.5\%), 110 (57.6\%), and 112 (58.6\%) MSUCOM students, respectively, rated dermatology of equal importance (i.e., 3/5). However, when asked about the value of having a sole dermatology course in pre-clerkship Osteopathic curriculum, 107 (56\%) MSUCOM students responded with "highly valuable" (i.e., 5/5).

Chi-square test demonstrated no difference $(\mathrm{P}>0.05)$ between the proportion of the students' rate for the importance of dermatology only vs. derm and the other course systems.

Conclusion: There is a questionable absence of dedicated dermatology education in the Osteopathic pre-clerkship curriculum in the United States. This is the first study investigating the importance of dermatology education relative to other systems courses in the Osteopathic preclerkship curriculum from medical students' perspectives. Our results demonstrate that dermatology education is valued by over $50 \%$ of the students with equal importance to the other course systems, which suggests implementation of a dedicated integumentary course in pre-clerkship curriculum would be well-received. The results gathered from our study support our hypothesis that medical students view dermatology as crucial towards their Osteopathic medical education so that they would be better trained to offer quality patient care as practicing physicians.Though we are limited to just MSUCOM medical students, our study is a foundational launching point to bring attention to the hole in our training that could negatively impact patients with undiagnosed/misdiagnosed dermatological conditions. As future Osteopathic physicians, we must practice the Osteopathic tenants and ensure that we are treating the patient as a whole. ${ }^{5}$ We hope this study encourages the implementation of dedicated dermatology education for all medical student's training. Further studies can be done to further generalize to the overall Osteopathic medical community as a whole.

\section{References}

1. Weishar, M (2014). Integrating dermatology education into the medical school curriculum: creation and evaluation of a clerkshipbased curriculum for third-year medical students. JAAD. 70(5). doi:10.1016/j.jaad.2014.01.323

2. Cahn, BA, et al. (2020) Current status of dermatologic education in US medical schools. JAMA Dermatology, 156:468-70. doi:10.1001/ jamadermatol.2020.0006

3. American Association of Colleges of Osteopathic Medicine (2020) Student Guide to OMC. https://choose do.org/wp-content/ uploads $/ 2020 / 08 / 2020-2021-S t u d$ ent-Guide-ACCESSIBLE-WEBAug17.pdf

4. Hansra, NK, et al. (2009). Medical school dermatology curriculum: are we adequately preparing primary care physicians? JAAD. 61: 23-9. doi:10.1016/j.jaad.2008.11.912

5. Austin E et al. (2005) Osteopathic medicine and the practice of dermatology: history and current status. J Am Acad Dermatol. 53:1047-52. doi: 10.1016/j.jaad.2005.08.016.

Financial Disclosures: None reported.

Support: None reported.

Ethical Approval: This study was reviewed and approved by the IRB on May 19, 2021. MSU IRB approval: STUDY 00006234.

Informed Consent: N/A

Poster No.: *B36

Abstract No.: 90

Category: Basic Science 
Research Focus Area: Chronic Diseases \& Conditions Perceived Stress and Perceptions of Vaginal Health Amongst Female Medical Students During COVID-19

Rachel Southard, OMS-III; Kristina P. Burger

Western University of Health Sciences College of Osteopathic Medicine of the Pacific (WesternU/COMP)

Context: Hormonal changes induced by stress cultivates a habitat that promotes pathogen growth in the vagina. As a result, infections can occur, which causes psychological stress leading to a vicious cycle of illness. ${ }^{1}$ Chronic vaginal infections impact physical and mental health and may also impact fertility. As some female medical students intend on bearing children, a combination of long-term stress from academic demands and chronic vaginal infections may pose barriers.

Objective: To examine the relationship between perceived stress among female medical students and its impact on vaginal health.

Methods: An anonymous, voluntary online survey was administered to cis females that are currently enrolled in medical school in the United States, who are not pregnant, and are over the age of 18 . An invitation to participate was posted on Rachel Southard's YouTube platform which reaches an estimate of 10,000 female medical students in the United States. The exclusion criteria include currently breastfeeding, currently pregnant, non-United States medical students, and individuals under the age of 18 . Of those who met the criteria and volunteered to participate, 1,057 women participated between 09/25/2020 and 10/25/ 2020. The twenty-eight, multiple choice question survey addressed exercise, diet, sexual practices, use of contraceptives, stress perception, and stress-coping methods over the previous 6 months. Data were analyzed through partial correlation and multiple regression analysis. The software, IBM SPSS Statistics V25 was used for analysis. As health is multidimensional, it is important to examine both internal and external factors that impact the body's selfregulating ability.

Results: A total of 1029 participants were included in the final analysis (response rate: 9.72\%). Partial correlation analysis revealed that vaginal health was correlated with sexual activities ( $\mathrm{r}=-0.056, \mathrm{p}=0.037)$, use of sex toys $(r=-0.089, p=0.002)$, having more than one sexual partner $(\mathrm{r}=-0.127), \mathrm{p}<0.001)$, systemic antibiotic use $(\mathrm{r}=-0.200$, $\mathrm{p}<0.001)$, hormonal contraceptive use $(\mathrm{r}=-0.109, \mathrm{p}<0.001)$, and perceived stress $(\mathrm{r}=0.112, \mathrm{p}<0.001)$. Multiple regression analysis found that vaginal health was predicted by the full model (adjusted $R 2=0.069, p<0.001)$, with stress $(\beta=0.128$, $\mathrm{p}<0.001)$, having more than one sexual partner $(\beta=-0.066$, $\mathrm{p}=0.040)$, systemic antibiotic use $(\beta=-0.163, \mathrm{p}<0.001)$, and oral sex $(\beta=-0.097, p=0.007)$ emerging as unique predictors of vaginal health.

Conclusion: There are many factors that influence vaginal health; however, perceived stress is an important element that is often neglected. While perceptions of vaginal health can be significantly predicted by the variables measured, only $6.9 \%$ of vaginal health can be predicted from this sample. Nonetheless, there are variables that contribute uniquely to perceptions of vaginal health. These include having multiple sexual partners, systemic antibiotic use, oral sex practices, and perceived stress.

\section{References}

1. Nansel TR, Riggs MA, Yu KF, Andrews WW, Schwebke JR, Klebanoff MA. The association of psychosocial stress and bacterial vaginosis in a longitudinal cohort. Am J Obstet Gynecol. 2006;194(2):381386. doi:10.1016/j.ajog.2005.07.047

Financial Disclosures: None reported.

Support: Summer Student Fellowship Grant from Western University of Health Sciences was awarded to Rachel Southard and Kristina Burger. In addition, an anonymous donor donated Amazon gift cards, which were raffled to participants as gratitude for their time.

Ethical Approval: The survey was approved by the Western University of Health Sciences Institutional Review Board on an exempt basis under listing number 1579676-2. Informed Consent: Informed consent was obtained through statements at the beginning of the survey declaring the criteria required to complete the survey and that the survey was both voluntary and anonymous. Submission of a completed survey was considered acceptance of informed consent to participate in the study.

Poster No.: B37

Abstract No.: 91

Category: Basic Science

Research Focus Area: Acute and Chronic Pain Management AOA Grant Award: 20146802 


\section{OMM alters chronic ethanol- induced changes to VTA GABA neurons, NAC DA release and measures of withdrawal in rats}

\author{
${ }^{1}$ Kyle Bills, PhD; ${ }^{2}$ Christina Small; ${ }^{2}$ Dallin Otteson; ${ }^{2}$ Gavin \\ Jones; ${ }^{3}$ Scott Steffensen, PhD \\ ${ }^{1}$ Brigham Young University; ${ }^{2}$ Department of Neuroscience, \\ Brigham Young University; ${ }^{3}$ Department of Neuroscience, \\ Brigham Young University and Noorda College of Osteo- \\ pathic Medicine
}

Context: Emerging mechanistic data suggests that stimulation to spinal mechanoreceptors may be effective in the treatment of opioid and ethanol-use disorders by modulating brain regions involved in chronic drug exposure. ${ }^{123}$ These modulations include robust changes in the mesolimbic circuitry. ${ }^{4} 5$ These data underlie the import of evaluating potential effects of osteopathic manual manipulation on the mesolimbic circuitry.

Objective: Frequency specific tissue displacement, as a reproducible analogue to cervical spine OMM (termed 'OMMa'), in rodents has been used to modify neuronal GABA activity in the ventral segmental area (VTA) and dopamine (DA) release in the nucleus accumbens (NAc). ${ }^{4,5}$ To determine whether OMMa to the cervical spine can ameliorate the effects of ethanol-induced changes to VTA GABA neurons, DA release in the NAc and behavioral measures of withdrawal in rats.

Methods: In this mechanistic original research we randomly assigned Wistar rats to one of four groups, saline no OMMa, saline OMMa, ethanol no OMMa and ethanol OMMa. Groups were given isovolumetric injections of ethanol $(2.5 \mathrm{mg} / \mathrm{kg}$; IP) or saline and either given OMMa or sham. OMMa was administered by a surgically implanted mechanical stimulation device adjacent to the right C3-7 laminae. Intervention was given immediately following injection 2x/day for 7 days to induce dependence. 24-hours post final injection, during withdrawal, behavioral assays were performed. Dopamine release and GABA neuron firing was measured during withdrawal following a reinstatement dose of ethanol $(2.5 \mathrm{mg} / \mathrm{kg}$; IP) with microdialysis and single unit electrophysiology respectively. Data were analyzed using ANOVA followed by Tukey post hoc analyses. The purpose of this study was to determine a mechanistic rationale for potential human testing of OMM in the treatment of alcohol use disorder.
Results: We show that when OMMa is administered concurrently with alcohol, it alters alcohol-induced desensitization of VTA GABA neuron firing rate in response to a reinstatement dose of ethanol $(2.5 \mathrm{mg} / \mathrm{kg} ; \mathrm{IP})$ from $117.5 \%$ of baseline to $32.3 \%$. Dopamine release in the $\mathrm{NAc}$ at 20 min post-injection was changed from $95.4 \%$ of baseline to $144.4 \%$ and at $80 \mathrm{~min}$ from $104.1 \%$ to $138.2 \%$. Further, behavioral indices of withdrawal (rearing, openfield crosses, tail stiffness, gait and elevated-plus times) were substantively ameliorated with concurrent OMMa treatment.

Conclusion: These data suggest a possible role for the use of OMM in the treatment of alcohol and opioid-use disorders. However, further studies are needed to evaluate hodological underpinnings of the findings presented and to evaluate proper frequency and timing of potential treatments. In all, this study demonstrates that further study in this area is indicated.

\section{References}

1. Steffensen SC, Stobbs SH, Colago EE, et al. Contingent and noncontingent effects of heroin on mu-opioid receptor-containing ventral tegmental area GABA neurons. Exp Neurol 2006;202(1):13951 doi: 10.1016/j.expneurol.2006.05.023[published Online First: Epub Date]|.

2. Gallegos RA, Criado JR, Lee RS, Henriksen SJ, Steffensen SC. Adaptive responses of GABAergic neurons in the ventral tegmental area to chronic ethanol. J. Pharmacol. Exp. Ther. 1999;291:1045-53

3. Hirose N, Murakawa K, Takada K, et al. Interactions among muand delta-opioid receptors, especially putative delta1- and delta2-opioid receptors, promote dopamine release in the nucleus accumbens. Neuroscience 2005;135(1):213-25 doi: 10.1016/j. neuroscience.2005.03.065[published Online First: Epub Date]|.

4. Bills KB, Obray JD, Clarke T, et al. Mechanical stimulation of cervical vertebrae modulates the discharge activity of ventral tegmental area neurons and dopamine release in the nucleus accumbens. Brain Stimul 2019 doi: 10.1016/j.brs.2019.11.012 [published Online First: Epub Date]|.

5. Bills KB, Clarke T, Major GH, et al. Targeted Subcutaneous Vibration With Single-Neuron Electrophysiology As a Novel Method for Understanding the Central Effects of Peripheral Vibrational Therapy in a Rodent Model. Dose Response 2019;17(1):1559325818825172 doi: 10.1177/1559325818825172[published Online First: Epub Date]|.

Financial Disclosures: None reported.

Support: The American Osteopathic Association and Departmental funding for supplies, animals, student stipends and protected faculty time from Noorda College of Osteopathic Medicine and Brigham Young University. 
Ethical Approval: All experiments were reviewed and approved by the IACUC under protocol number 18-1202. Informed Consent: N/A

Poster No.: ${ }^{\star} \mathrm{C} 1$

Abstract No.: 2

Category: Clinical

Research Focus Area: Osteopathic Philosophy

Analysis of Price Transparency via Mandated Standard Charge Listings for Routine Vaginal Delivery in Tennessee Hospitals

Lillie Campbell, OMS-III

Lincoln Memorial University DeBusk College of Osteopathic Medicine (LMU-DCOM)

Context: The cost of a vaginal birth in the United States saw an increase of $148 \%$ from 2008 to 2015 raising the maternal financial burden from $\$ 2910$ to $\$ 4314$ on average. The US Centers for Medicare \& Medicaid Services mandated that institutions licensed as hospitals were required to post their standard charges prominently on publicly available websites by January 1, 2021. This allows patients to look up the price of a vaginal delivery and consider financial burden as part of the birth planning process.

Objective: To investigate the price variation in one healthcare market in Tennessee using newly available information as mandated by the CMS price transparency rule and extrapolate the accuracy and ease of access of this information.

Methods: We identified the January 2021 publicly available standard charge listings for hospitals in eastern Tennessee. We isolated the charge corresponding to diagnosis related group 807 which codes for vaginal delivery without sterilization or D\&C and without comorbidity or complication. We then calculated the mean (SD) charge at all included hospitals. No human participants were included in this study.

We analyzed the degree of price variation and compared the mean price to the price paid by Medicare using the Geographic Practice Cost Index published in the 2021 Medicare Physician Fee Schedule.

We noted the importance of cost to patients when the focus is empathetic, whole-person health care.

Results: Of the 14 hospitals, the mean (SD) listed price for vaginal delivery as DRG 807 was $\$ 6,499.43(\$ 1,181.68)$ in January 2021. The prices ranged from $\$ 4,978.97$ to $\$ 9,529.40$, representing a difference by a factor of 1.9 . The mean listed price of $\$ 6,499.43$ was 2.7 times the price of $\$ 2,398.29$ that Medicare states it would pay based on the MPFS data.

Conclusion: The availability of CMS-mandated hospital standard charge listings and the nomenclature used for vaginal delivery are not uniform, and the listed prices are variable. The association between listed charges and actual prices paid by patients or their insurers is unclear, mitigating the value of the CMS rule for patients considering financial burden in their birth plans. This study suggests that although implementation of the CMS price transparency policy may be insufficient to enable patients to estimate or compare prices, it may play a role in disclosing a financial factor contributing to the increasing maternal mortality rate in the United States.

\section{References}

1. Moniz, M. H., Fendrick, A. M., Kolenic, G. E., Tilea, A., Admon, L. K., \& Dalton, V. K. (2020). Out-of-pocket spending for maternity care among women with employer-based insurance, 2008-15. Health Affairs, 39(1), 18-23,23A-23H. https://doi.org.Imunet.idm.oclc. org/10.1377/hlthaff.2019.00296

2. "To Help Fix The Maternal Health Crisis, Look To Value-Based Payment," Health Affairs Blog,July 16, 2019.DOI: 10.1377/ hblog20190711.816632

3. Health Care Transformation Task Force. (2019). Expanding Access to Outcomes-Driven Maternity Care through Value-Based Payment. https://hcttf.org/outcomes-driven-maternity-care-vbp/

4. How To Get the Most Out Of Hospital Price Transparency. https:// www.cms.gov/hospital-price-transparency/consumers

5. Skepticism about new US government hospital pricing transparency rule. (2019). Lancet Oncology, 20(2), 188. https://doi.org. Imunet.idm.oclc.org/10.1016/S1470-2045(19)30002-6

6. Agarwal, A., Dayal, A., Kircher, S. M., Chen, R. C., \& Royce, T. J. (2020). Analysis of Price Transparency via National Cancer Institute-Designated Cancer Centers' Chargemasters for Prostate Cancer Radiation Therapy. JAMA oncology, 6(3), 409-412. https:// doi.org/10.1001/jamaoncol.2019.5690

7. Cook, K., \& Loomis, C. (2012). The Impact of Choice and Control on Women's Childbirth Experiences. The Journal of perinatal education, 21(3), 158-168. https://doi.org/10.1891/1058-1243.21.3.158

Financial Disclosures: None reported.

Support: None reported.

Ethical Approval: No IRB approval is required.

Informed Consent: Not relevant.

Poster No.: C2

Abstract No.: 3

Category: Clinical 
Research Focus Area: Osteopathic Philosophy

Development of Osteopathic Neuromusculoskeletal Medicine (ONMM) Residency Curriculum Guidelines to meet ACGME Milestones

${ }^{1}$ Elizabeth Balyakina DO, MS, MPH, PGY-4; ${ }^{2}$ Malinda M. Hansen, DO, MS, CAQSM; ${ }^{2}$ David Mason, DO, MBA, FACOFP

${ }^{1}$ Medical City Fort Worth (MCFW) Hospital; ${ }^{2}$ Department of Family Medicine and Department of Osteopathic Manipulative Medicine, University of North Texas Health Science Center

Context: Residencies accredited through the American Osteopathic Association (AOA) completed merging with programs accredited through the Accreditation Council for Graduate Medical Education (ACGME) in 2020. ${ }^{1,2}$ This included the transition of Osteopathic Neuromusculoskeletal Medicine (ONMM) residencies. Progress through ONMM residency is evaluated based on fifteen ACGME milestones. ${ }^{3}$ However, there are no guidelines to help guide the achievement of these milestones as there are in other specialties. ${ }^{4-6}$

Objective: The primary purpose of this study was to develop a proposed structure and content for an ONMM residency curriculum (1) based on the alignment of residency curriculum with ACGME milestones in one ACGME accredited ONMM residency program, and (2) the perceived needs of residents and faculty for an ONMM residency curriculum.

Methods: A mixed methods exploratory sequential approach with embedded design was used.7 Qualitative analysis of didactics curriculum content for the past two years was hand coded according to themes identified in the residency curriculum content which were further coded according to ACGME milestones. ${ }^{3}$ Curriculum topics identified in qualitative analysis were used to create a questionnaire which was administered to residents and faculty $(\mathrm{n}=24)$ in the ONMM residency program to examine perceived importance of each curriculum topic based on a five-point Likert scale. Open-ended questions were embedded in the questionnaire that asked how faculty and residents define ONMM and what they believe should be the purpose of an ONMM residency curriculum.

Results: Five themes were identified in qualitative analysis of curriculum, namely, (1) OMM laboratory topics, defined as didactics topics that involve the hands-on application of psychomotor skills, (2) faculty-led activities and lecture topics, (3) resident-led activities and lecture topics, (4) research, and (5) training courses and volunteer activities. The most important perceived curriculum topics for faculty and residents were Osteopathic structural examination, orthopedic exam, direct and indirect methods, Osteopathic Cranial Manipulative Medicine, pediatric OMT, common upper and lower extremity injuries, and low back pain. Each of these topics aligned well with ACGME milestones. Residents reported that integrative medicine topics such as acupuncture were a significantly more important ONMM residency laboratory topic (mean $=3.58, \quad \mathrm{SD}=0.996$ ) compared to faculty (mean=2.33, $\mathrm{SD}=0.985), \mathrm{t}(22)=-3.091$, $\mathrm{p}=0.005$. Study participants most commonly described ONMM in terms of the specialized knowledge required for the discipline ( $\mathrm{n}=19,79.2 \%)$ and the Tenets of Osteopathy $(\mathrm{n}=17,70.8 \%)$, and felt that the purpose of an ONMM residency curriculum should be to gain knowledge $(n=20$, 83.3\%) and become a competent physician ( $\mathrm{n}=19,79.2 \%)$. Conclusion: Present findings were employed in the development of proposed ONMM residency curriculum guidelines and submitted to the American Academy of Osteopathy for consideration. They are presented here as a resource for application to ONMM residency curriculum. Where possible, it may be beneficial to adopt components of ONMM curriculum from existing evidence-based medical and post-graduate educational programs, and implement evaluation methodologies that ensure the appropriate application of curriculum to individual program needs. ${ }^{8-12}$

\section{References}

1. Ahmed AH, Schnatz PF, Adashi EY. Allopathic and Osteopathic medicine unify GME accreditation: a historic convergence. Fam Med. 2017;49(5):374-377.

2. Accreditation Council for Graduate Medical Education. Frequently Asked Questions: Single Accreditation System. https://acgme. org/Portals/0/PDFs/Nasca-Com munity/FAQs.pdf. Accessed on September 6, 2020.

3. Sharp H, Carnes M, Edgar L, Kisiel S, Leuenberger J, et al. The Osteopathic Neuromusculoskeletal Medicine milestone project; 2015. https://www.acgme.org/Portals/0/PDFs/Milestones/ OsteopathicNeuro musculo skeletalMedicnieMilestones.pdf? ver=2016-09-02-105148-897. Accessed on April 18, 2021.

4. American Academy of Family Physicians. Family medicine residency curriculum guidelines. https://www.aafp.org/studentsresidents/residency-program-directors/curriculum-guidelines. html. Accessed on September 6, 2020.

5. Clerkship Directors in Internal Medicine/Society of General Internal Medicine. Core medicine clerkship curriculum guide: a 
resource for teachers and learners, version 3.0; 2006. https:// higherlogicdownload.s3.amazonaws.com/IM/fecab58a-0e31416b-8e56-46fc9e da5c37/UploadedImages/Documents/Online CDIMCurriculum.pdf. Accessed on April 18, 2021.

6. Surgical Council on Resident Education. Curriculum outline for general surgery; 2020-2021. http://files.surgicalcore.org/20202021_GS_CO_Booklet_v3editsfinal.pdf. Accessed on April 18, 2021.

7. Creswell JW. A Concise Introduction to Mixed Methods Research. Thousand Oaks, California: SAGE Publications, Inc.; 2015.

8. Thomas PA, Kern DE, Hughes MT, Chen BY, ed. Curriculum Development for Medical Education: A Six-Step Approach, Third Edition. Baltimore, Maryland: John Hopkins University Press; 2016.

9. Cochran N CP, Reed V, Thurber P, Fisher E. Beyond fight or flight: the need for conflict management training in medical education. Confl Resolut Q. 2018;35:393-402.

10. Furney SL, Orsini AN, Orsetti KE, Stern DT, Gruppen LD, et al. Teaching the one-minute preceptor: a randomized controlled trial. J Gen Intern Med. 2001;16(9):620-624.

11. Leis JA, Shojania KG. A primer on PDSA: executing plan-do-studyact cycles in practice, not just in name. BMJ Qual \& Saf. 2017;26(7):572-577.

12. Harrell H, Wipf J, Aronowitz P, Rencic J, Smith DG, et al. Resident as teacher curriculum. MedEdPORTAL; 2015. https:// doi.org/10.15766/mep_2374-8265.10001. Accessed on April 18, 2021.

Financial Disclosures: None reported.

Support: This work was supported in whole or in part by a grant from the Texas Higher Education Coordinating Board (THECB). The opinions and conclusions expressed in this document are those of the author(s) and do not necessarily represent the opinions or policy of the THECB.

This research was supported (in whole or in part) by HCA and/or an HCA affiliated entity. The views expressed in this publication represent those of the author(s) do not necessarily represent the official views of HCA or any of its affiliated entities.

Ethical Approval: The study protocol was submitted under CARRIE (Centralized Algorithms for Research Rules on IRB Exemption) through Medical City Fort Worth; the project was determined to be exempt from Institutional Review Board oversight (IRB reference\# 2020-753) on October 1, 2020.

Informed Consent: The survey portion of the study was administered online via Qualtrics between October 21, 2020 and November 12, 2020. Potential study participants were emailed a link to complete the survey with three subsequent reminder emails. Potential participants were asked to complete a consent form on Qualtrics. Individuals not wishing to complete the survey could simply close the link.
Poster No.: ${ }^{\star} \mathrm{C} 3$

Abstract No.: 17

Category: Clinical

Research Focus Area: Impact of OMM \& OMT

\section{The Effect of Counterstrain}

Technique on Muscle Stiffness and

Pain on Trapezius Tender Points in Medical Students

${ }^{1}$ Richard Liang, OMS-III; ${ }^{2}$ Sammi Wong, OMS-III; ${ }^{2}$ Kevin Song, OMS-III; ${ }^{2}$ Lerone Clark, OMS-II; ${ }^{2} J a i$ Joshi, OMS-II; ${ }^{2}$ Aziz Ur Rahman Khalid, OMS-II; ${ }^{3}$ Min-Kyung Jung, PhD; Sheldon Yao, DO, FAAO; ${ }^{4}$ Philip Noto, DO, FAAPMR

${ }^{1}$ New York Institute of Technology (NYITCOM); ${ }^{2}$ Department of Osteopathic Manipulative Medicine, NYIT College of Osteopathic Medicine; ${ }^{3}$ Department of Research, NYIT College of Osteopathic Medicine; ${ }^{4}$ Department of Osteopathic Manipulative Medicine, NYIT College of Osteopathic Medicine;

Context: Medical students often exhibit poor studying posture, which can result in increased muscle stiffness surrounding the neck, pain and manifest into tender points. Previous studies on Counterstrain (CS) techniques have shown efficacy in decreasing pain scores. A device called the MyotonPRO has been used to assess muscle tone after non-CS osteopathic techniques. However, to date, no study has established a significant relationship between CS tender points and muscle tonicity using a MyotonPRO.

Objective: The primary aim of this study was to investigate the efficacy of CS technique in decreasing pain in trapezius tender points in medical students. The secondary aim was to utilize the MyotonPRO device to determine if CS technique changes muscle tone in the treated tender points.

Methods: Twenty-seven medical students (17 females and 10 males) were evaluated for tender points in the upper trapezius bilaterally by a board-certified faculty member in the OMM department. The side with the highest initial pain rating was labeled as the treatment side, whereas the other side was labeled as the control side. A student investigator, who was blinded to the treatment and control trapezius tender points, measured all parameters, including muscle tone, using the MyotonPRO, bilaterally. The physician treated the treatment side with CS technique. Subjective reports of pain rating (out of 10) were recorded for the treatment side both pre and post-treatment. The points were measured again post-treatment by the blinded student investigator using the MyotonPRO, bilaterally. Pain 
scale changes and muscle stiffness data collected by the MyotonPRO was analyzed using SPSS statistical software. Results: The mean change in subjective pain scores before and after CS was 4.9 with a p-value of $<0.001$, indicating a statistically significant difference. The mean change in muscle stiffness measured by the MyotonPRO before and after CS treatment was $4.9 \mathrm{~N} / \mathrm{m}$ with a p-value of 0.09 , indicating a statistically insignificant difference.

Conclusion: This study demonstrated that CS was effective in reducing pain levels in trapezius tender points in medical students. Further research may be needed regarding the use of MyotonPRO in measuring the physiological parameters of muscle in both pre- and post-counterstrain treatment. Further investigation is warranted regarding whether physiological changes in the trapezius muscle, as measured by the MyotonPRO, can be adequately correlated to the test subject's decrease in subjective pain scores.

\section{References}

1. Du, J. Y., Aichmair, A., Schroeder, J. E., Kiely, P. D., Nguyen, J. T., \& Lebl, D. R. Neck Pain and Low Back Pain in Medical Students: A Cross-Sectional Study. International Archives of Public Health and Community Medicine. 2017; doi: 10.23937/IAPHCM-2017/1710002

2. Borg-Stein, J., \& Stein, J. Trigger points and tender points: one and the same? Does injection treatment help?. Rheumatic Disease Clinics. 1996. 22(2), 305-322. doi: 10.1016/s0889857x(0570274-x)

3. Seffinger, M. A. Foundations of osteopathic medicine: philosophy, science, clinical applications, and research. Philadelphia: Lippincott Williams \& Wilkins. 2018. 763-783, 864-884.

4. Kisilewicz, A., Urbaniak, M., \& Kawczyński, A. Effect of muscle energy technique on increased calf muscle stiffness after eccentric exercise in athletes. Journal of Kinesiology and Exercise Sciences. 2018. 81(28), 21-29. doi: 10.5604/01.3001.0012.7985

Financial Disclosures: None reported.

Support: None reported.

Ethical Approval: This study was approved by the New York Institute of Technology Institutional Review Board under Protocol \#BHS-1561.

Informed Consent: Research subjects were informed of the purpose, description, potential risks and potential benefits of the research study by a member of the research team. Research subjects then signed an informed consent form after being given adequate time to ask any questions they may have. All health information of research subjects remained confidential and accessed only by members of the research team. Research subjects voluntarily agreed to participate in this study and were given the option to withdraw from the study at any time without penalty or loss of benefits.

Poster No.: ${ }^{\star} \mathrm{C} 4$

Abstract No.: 20

Category: Clinical

Research Focus Area: Chronic Diseases \& Conditions

Metabolic Profile of Women with Hypermobile Ehlers-Danlos Syndrome/Hypermobility Spectrum Disorders compared to Age Matched Controls.

${ }^{1}$ Madeline Margulies OMS-III; ${ }^{2}$ Bernadette Riley, DO; ${ }^{3}$ MinKyung Jung, PhD; ${ }^{4}$ Veronica Southard, PT, DHSc; ${ }^{5}$ Joanne DiFrancisco-Donoghue, PhD;

${ }^{1}$ New York Institute of Technology (NYITCOM); ${ }^{2}$ Department of Family Medicine, New York Institute of Technology College of Osteopathic Medicine; ${ }^{3}$ Department of Research, New York Institute of Technology College of Osteopathic Medicine; ${ }^{4}$ Department of Physical Therapy, New York Institute of Technology School of Health Professions; ${ }^{5}$ Department of Osteopathic Manipulative Medicine, New York Institute of Technology College of Osteopathic Medicine

Context: Hypermobile Ehlers-Danlos Syndrome (hEDS) and Hypermobility Spectrum Disorders (HSD) are associated with musculoskeletal pain, decreased bone mineral density (BMD) and gastrointestinal (GI) complications ${ }^{1,2,3}$. Though the etiology of low BMD may be multi-factorial, the role of GI symptoms and diet in BMD has not been established in this population. This study investigates how GI distress influences nutritional intake and the effect it may have on BMD and body composition in this rare disorder. Objective: The primary objective of this study is to determine the frequency and severity of GI symptoms compared to body composition and bone mineral abnormalities in individuals with hEDS and HSD. The secondary objective is to examine the effect of GI symptoms on energy balance and body composition.

Methods: This pilot study was approved by the New York Institute of Technology College of Osteopathic Medicine (NYITCOM) Institutional Review Board. This was an observational study that recruited $10 \mathrm{hEDS} / \mathrm{HSD}$ subjects age $28 \pm 4$, Body Mass Index (BMI) $21 \pm 3$ from the NYITCOM Ehlers-Danlos Syndrome (EDS) Center and 10 age, sex, and BMI matched controls (age $27 \pm 4$, BMI $22 \pm 2$ ) that were 
recruited from the local community. Subjects signed written consent to participate in this study. Inclusion criteria required a Beighton score of $\geq 4$. Excluded were anyone with other forms of EDS, other forms of connective tissue diseases and pregnancy. Subjects recorded mealtime, meal composition, and the amount of food consumption for 2 days. The macronutrient breakdown and caloric intake was calculated in an online application. The Gastrointestinal Symptom Rating Scale (GSRS) is a validated diseasespecific instrument that consists of 15 questions combined into five symptom groups: reflux, abdominal pain, indigestion, diarrhea, and constipation. Dual-Energy X-ray absorptiometry (DXA) imaging was utilized to determine body composition including lean body mass (LBM), body fat $\%$ and BMD. Data was analyzed using a Mann Whitney U Test and a Spearman's Rho Correlation in the Statistical Package for the Social Sciences (SPSS) (alpha=0.05). The results from this study will assist osteopathic physicians in treating the whole patient by helping guide future clinical recommendations for diet and therapy in this rare population.

Results: There was no correlation between GSRS score and BMD ( $r s=0.06, p=0.82, n=20$ ). There was no significant difference in caloric intake and fat consumption between hEDS/HSD and controls ( $p=0.58 ; p=0.28)$. However, hEDS/ HSD subjects consumed a significantly lower amount of protein and a significantly higher amount of carbohydrates $(\mathrm{p}=0.002 ; \mathrm{p}=0.023)$. The hEDS/HSD subjects reported higher GI symptoms on the GSRS compared to the controls $(p<0.001)$. Interestingly, GSRS was negatively correlated to protein intake $(\mathrm{rs}=-0.57, \mathrm{p}=0.019, \mathrm{n}=20$ ). Therefore, the greater GI symptoms reported, the lower amount of protein the subject consumed. No difference in BMD, LBM or body fat between the hEDS/HSD and healthy controls were found ( $\mathrm{p}=0.28 ; \mathrm{p}=1.0 ; \mathrm{p}=0.39$ ).

Conclusion: This pilot study has identified that women with hEDS/HSD presented with a deficit in protein intake, which correlated with increased GI complications that are associated with hEDS/HSD. Although no difference was found between BMD, LBM and fat mass in this cohort, the reduced protein intake long-term may have a lasting impact on bone and muscle health. These results may be due to the small sample size. The data is still being collected in a larger cohort of subjects. The information from this project will help physicians gain a better understanding of the relationship between GI symptoms, diet, and BMD in this population. Future studies will need to be conducted to understand the longitudinal effects of a protein deficit in this rare disease and to help guide future preventive and nutritional treatment approaches in individuals with hEDS and HSD.

\section{References}

1. Eller-Vainicher, C. et al. Bone involvement in adult patients affected with Ehlers-Danlos syndrome. Osteoporos Int 27, 25252531, doi:10.1007/s00198-016-3562-2

2. Fikree, A., Chelimsky, G., Collins, H., Kovacic, K. \& Aziz, Q. Gastrointestinal involvement in the Ehlers-Danlos syndromes. Am J Med Genet C Semin Med Genet 175, 181-187, doi:10.1002/ajmg.c. 31546

3. Evans, W. J. Skeletal muscle loss: cachexia, sarcopenia, and inactivity. Am J Clin Nutr 91, 1123S-1127S, doi:10.3945/ajcn.2010. 28608A

Financial Disclosures: None reported.

Support: This study was funded by the Marfan Foundation Research Grant (Grant \#: 589241). Research subjects were compensated for their time.

Ethical Approval: This study was reviewed and approved by the New York Institute of Technology College of Osteopathic Medicine Institutional Review Board. IRB \#: BHS-1453

Informed Consent: Subjects read the consent form and were given an explanation of the study's risks and purpose. Subjects were given the opportunity to ask questions. Subjects were consented with a witness, the principle investigator.

Poster No.: ${ }^{\star} \mathrm{C} 5$

Abstract No.: 21

Category: Clinical

Research Focus Area: Impact of OMM \& OMT

Patient Perception of Clinical Trials With and Without Osteopathic Manipulative Treatment During the COVID-19 Pandemic

${ }^{1}$ Jacqueline Nikakis MBS, OMS-II; ${ }^{2}$ Uddampreet Singh Arora, OMS-II; ${ }^{2}$ Leana Wang, OMS-II; ${ }^{2}$ Chiya Abramowitz, OMS-II; ${ }^{2}$ Denis Malkov, OMS-II; ${ }^{2}$ Todd J. Cohen, MD

${ }^{1}$ New York Institute of Technology (NYITCOM); ${ }^{2}$ Department of Clinical Specialties, New York Institute of Technology College of Osteopathic Medicine

Context: During the COVID-19 pandemic, many medical facilities that were providing hands-on therapy, such as Osteopathic Manipulative Therapy (OMT), had to discontinue or limit operations for safety purposes. Not much is known about whether the resumption of hands-on treatment has been met with enthusiasm, reluctance, or 
indifference by patients, and if these dispositions are influenced by vaccination or infection status.

Objective: To test the hypothesis that patients are more hesitant to participate in clinical trials with and without OMT during a pandemic as opposed to before the pandemic. In addition, this study will test the hypothesis that perceived immunity (through prior COVID-19 infection or vaccination) will enhance agreement to participate in clinical trials with or without OMT.

Methods: Clinical trials are an important tool used to understand the effects OMT has on human physiology. Following the COVID-19 pandemic, patients may have increased reluctance to receive OMT. To study this potential effect, 35 surveys were handed out to patients at the Long Island Heart Rhythm Center (LIHRC) at NYIT College of Osteopathic Medicine between June and July of 2021. The surveys were used as a screening tool for the ongoing IRB-approved study Effects of Osteopathic Manipulative Therapy on Arrhythmias (BHS-1464). In our survey study, cardiology patients answered five questions: (1) Have you ever tested positive for COVID-19? (2) Have you received at least one dose of a COVID-19 vaccine? (3) Are you willing to participate in a clinical trial? (4) Are you willing to participate in a clinical trial with Osteopathic Manipulative Therapy from a physician? (5) If you answered "No" or "Maybe" to question \#4, would you have participated in a clinical trial with hands-on treatment from a physician before the onset of the COVID-19 pandemic? The answer choices for questions 1 and 2 were "Yes" or "No," and for questions 3-5 the answer choices were "Yes," "No," or "Maybe." Additionally, under questions 3-5 there was a section to leave comments if the patient chose "Maybe." A quantitative analysis was performed on the data to make conclusions about patient outlook on clinical and OMT trials. Results: Out of the 35 patients surveyed, 29 patients received at least one dose of a COVID-19 vaccine, 6 patients previously contracted COVID-19, 3 patients did not have a COVID-19 vaccination or previous infection, and 2 patients received a COVID-19 vaccination and previously contracted COVID-19. 20 patients were willing to participate in a clinical trial, 21 were willing to participate in a clinical trial with OMT, and 29 would have participated in a clinical trial with OMT before the pandemic.

Previously Infected $(n=6): 5$ patients were willing to participate in a clinical trial, and 4 were willing to participate in a trial with OMT. 2 patients would not participate currently or prior to the pandemic.

Not Previously Infected $(n=29): 15$ patients were willing to participate in a clinical trial, and 17 were willing to participate in a trial with OMT. Out of the 12 patients who were unsure or would not currently participate, 8 reported that they would have participated in an OMT trial prior to the pandemic.

Vaccinated ( $\mathrm{n}=28$, includes patients who have also tested positive for COVID-19):

15 patients were willing to participate in a clinical trial, and 17 were willing to participate in a trial with OMT. Out of the 13 patients who were unsure or would not currently participate, 7 reported that they would have participated in an OMT trial prior to the pandemic.

Not vaccinated $(n=7): 5$ patients were willing to participate in a clinical trial, and 4 were willing to participate in a trial with OMT. Out of the 3 patients who were unsure or would not currently participate, 1 reported that they would have participated in an OMT trial prior to the pandemic.

Conclusion: This study demonstrates that more than half of the cardiology clinic patients surveyed were willing to participate in clinical trials with and without OMT. More than 20 percent of the participants that would not currently participate would have participated in a clinical trial with OMT before the pandemic.

Preliminary results appear to indicate that there is no difference between patients' willingness to participate in clinical trials with and without OMT, regardless of vaccination and previous COVID-19 infection status. Two patients indicated concern over clinical trials involving new medications and dangerous procedures, however, they were willing to participate in an OMT clinical trial.

74 percent of patients in our survey were vaccinated and did not have a previous COVID-19 infection. The sample size of patients without vaccinations and/or prior COVID-19 infection was too small for a statistical comparison. Additional patient recruitment is needed in order to truly determine whether there is a difference in participation in clinical trials between those with perceived immunity and those without. Further recruitment is also necessary to assess the effect of the COVID-19 pandemic on patients' dispositions towards participating in both clinical and OMT trials.

\section{References}

1. Ivy, C. C., Doerrer, S., Naughton, N., Priganc, V. (2021). The impact of COVID-19 on hand therapy practice. Journal of Hand Therapy. ISSN 0894-1130, https://doi.org/10.1016/j.jht.2021.01.007

2. Meijer, L. L., Hasenack, B., Kamps, J., Mahon, A., Titone, G., Dijkerman, H. C., \& Keizer, A. (2021, June 8). Out of touch: Touch deprivation and affective touch perception during the COVID-19 pandemic. https://doi.org/10.31234/osf.io/peq7m 
Financial Disclosures: None reported.

Support: None reported.

Ethical Approval: This survey was used as a screening tool used to enroll patients in an ongoing IRB-approved clinical trial (BHS-1465). ClinicalTrials.gov Identifier: NCT0400 4741. https://clinicaltrials.gov/ct2/show/NCT04004741? term $=$ BHS-1464\&draw $=2 \&$ rank $=1$

Informed Consent: Patients verbally consented to participate in the survey.

Poster No.: ${ }^{\star} \mathrm{C} 6$

Abstract No.: 30

Category: Clinical

Research Focus Area: Chronic Diseases \& Conditions

\section{Dementia Screening Effectiveness} in a Suburban Family Medicine Clinic

${ }^{1}$ Nguyen Huynh Pham OSM-III; ${ }^{2}$ Samantha Easterly, MA,

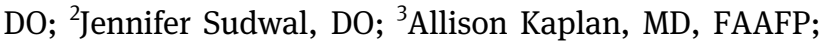
${ }^{4}$ Pamela Potter, $\mathrm{PhD}$

${ }^{1}$ Midwestern University Arizona College of Osteopathic Medicine (MWU/AZCOM); ${ }^{2}$ Family Medicine Residency, Mountain Vista Medical Center Residency Program; ${ }^{3}$ Family Medicine, Mountain Vista Medical Center Residency Program; ${ }^{4}$ Arizona College of Osteopathic Medicine, Midwestern University

Context: Early dementia detection offers benefits for patients' long-term health, treatment options, quality of life, and financial cost. However, there is a great conflict amongst dementia screening guidelines, especially in primary care settings. Therefore, there is a growing need for establishing a uniform set of screening guidelines as well as improving primary care providers' ability to recognize dementia and utilize assessment tools that are available to administer in the primary care setting.

Objective: The objective of this cohort study is to determine the extent of dementia screening of patients over the age of 65 in a primary care setting at a suburban family medicine clinic, and to track the rate of return for further evaluation.

The process improvement for family medicine will be to add research that helps balance the risks and benefits of dementia screening.

Methods: In a suburban family medicine clinic, 182 Medicare annual wellness visits of patients over 65 yearsold were reviewed. These visits occurred between 02/01/ 2020 and $07 / 31 / 2020$.
Data collected included demographics, Mini-Cog screening results with scoring by Peterson's criteria, selfreported memory problems, PHQ-9 score for depression evaluation, average pain score, follow-up recommendations, and treatment/management.

Standard of care for primary dementia screening is the Mini-Cog: a 3-word recall test, and a clock-test. It is $99 \%$ sensitive and $93 \%$ specific. $^{1,2}$

Results: - Out of the total 182 charts reviewed, 122 patients (67\%) screened negative, 14 (7\%) screened positive, and 46 (25\%) were not screened.

- The median age of screening was 73. The median of those that screened positive was 79 , whereas the median age screening negative was $72.5(\mathrm{p}=0.012)$.

- Return for further evaluation was recommended for $25(13.7 \%)$ patients. Of those, 9 screened positive, 8 screened negative, and 8 were not screened.

- Logistic regression analysis indicated that patients screening positive were more likely to return for further evaluation compared to patients not tested (OR 40.2, 95\% $\mathrm{Cl}$ 4.51-358.3) $(\mathrm{p}=0.001)$, or screened negative (OR 26.6, 95\% Cl 3.61-196.4) $(\mathrm{p}=0.001)$.

Conclusion: The median age of patients who screened positive for cognitive impairment by Peterson's criteria was 7.5 years older than those who screened negative. This is consistent with epidemiology showing an exponential increase in the prevalence and incidence of dementia from age 65 and onwards. ${ }^{3}$

Unfortunately, $25 \%$ of patients who were not screened represent a population where a diagnosis of dementia could be missed. Potential contributing factors for this finding include: the availability of various conflicting guidelines, providers' lacking the ability in screening for cognitive impairment properly, under-utilizing the tools available for screening, and hesitancy to proceed with dementia diagnosis. ${ }^{4,5}$

Out of 14 patients who screened positive at their initial visit, only 9 patients were recommended to return. Our future goal is to identify the specific factors contributing to this discrepancy, and perhaps initiate an internal quality improvement project to improve competence in dementia diagnosis and care amongst primary care providers.

Risks of screening: Evidence supports less is more with medical evaluation, and those who screened positive were advised to return for further testing. ${ }^{6}$ With the screen being a survey, there wasn't any reported harm to the patients who participated in it.

Benefits of screening: Patients screening positive were more likely to return for further evaluation compared to patients not tested (OR 40.2), or screened negative (OR 
26.6). This proves that proper screening at annual wellness visit is critical to address patients and caregivers' denial of cognitive problems and resistance to further evaluation. ${ }^{6}$

In conclusion, we identified a significant need for clear and reliable diagnostic guidelines to decrease the prevalence of missed and late diagnosis of dementia. It is important to improve providers' ability in assessing risk factors, diagnosing dementia before complications occur, and establishing a patient-centered care plan.

\section{References}

1. Ebell MH. Brief screening instruments for dementia in primary care. Am Fam Physician. 2009;79(6):497-500.

2. Tsoi KK, Chan JY, Hirai HW, Wong SY, Kwok TC. Cognitive Tests to Detect Dementia: A Systematic Review and Meta-analysis. JAMA Intern Med. 2015; 175(9):1450-1458. doi:10.1001/jamainternmed. 2015.2152

3. Alzheimer's Association. 2019 Alzheimer's Disease Facts and Figures. Alzheimers Dement 2019;15(3):321-87

4. Krogsbøll LT, Jørgensen KJ, Gøtzsche PC. General health checks in adults for reducing morbidity and mortality from disease. Cochrane Database Syst Rev. 2019;1(1):CD009009. Published 2019 Jan 31. doi:10.1002/14651858.CD009009.pub3

5. Bahar-Fuchs A, Martyr A, Goh AMY, Sabates J, Clare L. Cognitive training for people with mild to moderate dementia. Cochrane Database Syst Rev. 2018;2018(7):CD013069. Published 2018 Jul 6. doi:10.1002/14651858.CD013069

6. Falk N, Cole A, Meredith TJ. Evaluation of Suspected Dementia. Am Fam Physician. 2018;97(6):398-405.

Financial Disclosures: None reported.

Support: None reported.

Ethical Approval: This study was reviewed and approved.

IRB number: AZ 1382

Informed Consent: None.

Poster No.: ${ }^{\star} \mathrm{C} 7$

Abstract No.: 32

Category: Clinical

Research Focus Area: Chronic Diseases \& Conditions

\section{Vaccination and COVID-19: Pro- vider's Perspective}

\author{
${ }^{1}$ Jonathan Junqua OMS-IV; ${ }^{2}$ Geetika Sengupta \\ ${ }^{1}$ Touro University College of Osteopathic Medicine-CA \\ (TUCOM); ${ }^{2}$ Primary Care Department, Touro University \\ College of Osteopathic Medicine-CA (TUCOM)
}

Context: Vaccination is a crucial public health initiative for the health of youth worldwide. Vaccination ordering and administration fell dramatically in the age group under 24 months of age with the advent of COVID-19 ${ }^{[1]}$ and the biggest barrier to vaccination was the decrease in-person visits due to parental fear of COVID19 ${ }^{[2]}$. This pilot study aims to provide more context via physician practice-based perspectives to the low vaccination rates observed.

Objective: To assess changes in patient volume, percent and type of appointments cancelled, and vaccine hesitancy or anti-vaccine sentiments from before to during the COVID19 pandemic. To explore interventions conducted by providers or employers to encourage patient appointment attendance.

Methods: This is a survey based study, in which 64 participants were recruited through convenience sampling using a link and brief study explanation posted on physician and residency groups on social media or sent in a mailing list. Physicians specializing in pediatrics, family medicine or $\mathrm{OB} / \mathrm{GYN}$ were contacted between the months of September and December 2020. The inclusion criteria for the study were: 1) Participants must be healthcare providers who currently practice in the United States, 2) The participants provide vaccinations on a regular basis.

Chi-square test for independence was used for questions pertaining to patient volume and types of appointments cancelled. Remaining questions concerning percent of appointments cancelled by patients/providers and vaccine hesitancy were analyzed using analysis of variance testing with post hoc tests for statistically significant results. Three time points were examined for each question: 1) Before COVID19; 2) At lowest patient volume; 3) At the time of survey completion. Statistical significance was set at a $\mathrm{p}$-value of under 0.05. Free response answers for interventions or changes providers and/or employers implemented to encourage patients to attend their appointments were grouped and categorized into themes by following abridged steps outlined by Nowell et al. ${ }^{[3]}$. Responses could include more than one theme each, and the total frequency of each theme and sub-category was calculated.

The osteopathic significance of this study centers around the components of mind and spirit for physicians and patients. Adherence to public health and healthcare recommendations revolve largely around issues of trust, social contexts, and belief systems. By understanding the motivations and context of decreased vaccination in the setting of the COVID19 pandemic, providers will be better able to overcome these obstacles in the future.

Results: Out of 64 total respondents 8 respondents were excluded, resulting on 56 complete responses. Respondents practiced in 24 states, with the most responses coming from California (19), New York (4), and Texas (3). 
Most responses came from pediatricians (53) working in private or group practices (34).

Chi-square test for independence found a statistically significant decrease from pre-COVID19 patient volumes to lowest patient volumes as well as patient volumes at the time of survey completion ( $\mathrm{p}<0.0001$ for both comparisons). In addition, significant differences in types of appointments cancelled by patients/guardians versus providers were found $(\mathrm{p}<0.001)$, with providers cancelling more illness visits than guardians and providers cancelling less well-child checks below 7 years of age than guardians.

Analysis of variance testing found higher percentages of no-shows/cancellations by patients/guardians at the time of lowest patient volume versus pre-COVID19 $(\mathrm{t}=-3.5$, $\mathrm{p}<0.001)$ and lower percentages at the time of survey completion versus lowest patient volume $(t=2.17, p<0.05)$. The percent of cancellations by physicians/employers was higher at lowest patient volumes and at the time of survey completion versus pre-COVID19 $(\mathrm{t}=-5.12, \mathrm{p}<0.0001 ; \mathrm{t}=$ $-2.57, \mathrm{p}<0.05$ respectively), and lower cancellation percentages were found at the time of survey completion versus at lowest patient volume $(\mathrm{t}=4.05, \mathrm{p}<0.0005)$. Finally, no significant difference was found in the percent of guardians refusing vaccines or requesting alternative vaccination schedules $(\mathrm{F}=0.11, \mathrm{p}=0.89)$.

40 free responses were received detailing interventions or practice changes implemented to encourage appointment attendance. The most popular intervention theme was Reminders (found in 26 responses) followed closely by the theme of Practice Change (20 responses). Of the 40 total responses, nine of them used multiple interventions to encourage patients.

Conclusion: A sustained decrease from pre-COVID19 patient volumes was found versus those at lowest patient volumes and at the time of survey completion. This may be due to practice changes like socially distanced waiting rooms or continued parental fear of COVID19 ${ }^{[4]}$. The increase in appointment no-show/cancellation percentages by patients/guardians and providers may be contributing to the decrease in patient volumes. However, the percentages are returning to pre-COVID19 levels for patients/ guardians.

Providers/employers appear to be following AAP guidelines to prioritize in-person well-child checks ${ }^{[5]}$. Less well-child checks under 7 years old were cancelled by providers/employers than expected compared to guardians. Greater cancellation of illness visits by providers/ employers may be due to practice changes like the separation of well-child visits from illness visits.
Although there was no change found in the percent of patients/guardians refusing vaccines or requesting alternative vaccination schedules from before and during the pandemic, a separate study found that were differences in vaccine attitude changes based on political identity between March to August $2020{ }^{[6]}$, indicating the possibility that question specificity or study power may have been lacking to measure any changes that occurred.

Thematic analysis of free response questions revealed the theme of reminders to be most prevalent. Of note, automated or clinic staff based systems were most often used, which have been found to increase appointment attendance ${ }^{[7]}$. Practice based changes were also prevalent, and reflected similar interventions mentioned in a recent study ${ }^{[8]}$. The low number of responses mentioning multitheme interventions illustrates the potential for future research in its efficacy and cost-effectiveness. Limitations of this pilot study include the lack of generalizability, high likelihood of low response rate, and low representation of family medicine physician in the sample.

\section{References}

1. Santoli, J. M., Lindley, M. C., DeSilva, M. B., Kharbanda, E. O., Daley, M. F., Galloway, L., Gee, J., Glover, M., Herring, B., Kang, Y., Lucas, P., Noblit, C., Tropper, J., Vogt, T., \& Weintraub, E. Effects of the COVID-19 Pandemic on Routine Pediatric Vaccine Ordering and Administration-United States, 2020. MMWR. Morbidity and Mortality Weekly Report, 2020; 69(19), 591-593. Accessed January 12, 2021. doi: 10.15585/mmwr.mm6919e2

2. O’Leary, S. T., Cataldi, J., Lindley, M. C., Beaty, B. L., Hurley, L. P., Crane, L. A., Brtnikova, M., Gorman, C., Vogt, T., Kang, Y., \& Kempe, A. US Primary Care Providers' Experiences and Practices Related to Routine Pediatric Vaccination During the COVID-19 Pandemic. Centers for Disease Control and Prevention. Published March 23, 2021. Accessed May 9, 2021. https://www. cdc.gov/vaccines/hcp/pediatric-practices-during-COVID-19. html

3. Nowell, L. S., Norris, J. M., White, D. E., \& Moules, N. J. Thematic Analysis: Striving to Meet the Trustworthiness Criteria. International Journal of Qualitative Methods, 2017; 16(1), 1-13. Accessed May 5, 2021. doi: 10.1177/1609406917733847

4. Suffren, S., Dubois-Comtois, K., Lemelin, J.-P., St-Laurent, D., \& Milot, T. Relations between Child and Parent Fears and Changes in Family Functioning Related to COVID-19. International Journal of Environmental Research and Public Health, 2021; 18(4). Accessed May 9, 2021. doi: 10.3390/ijerph18041786

5. American Academy of Pediatrics. Guidance on Providing Pediatric Well-Care During COVID-19. American Academy of Pediatrics website. May 8, 2020. Accessed May 9, 2021. https://services. aap.org/en/pages/2019-novel-coronavirus-covid-19-infections/ clinical-guidance/guidance-on-providing-pediatric-well-care-during-covid-19/ 
6. Fridman, A., Gershon, R., \& Gneezy, A. COVID-19 and vaccine hesitancy: A longitudinal study. PLOS ONE, 2021; 16(4). Accessed May 10, 2021. doi: 10.1371/journal.pone. 0250123

7. McLean, S., Booth, A., Gee, M., Salway, S., Cobb, M., Bhanbhro, S., \& Nancarrow, S. Appointment reminder systems are effective but not optimal: Results of a systematic review and evidence synthesis employing realist principles. Patient Preference and Adherence, 2016; 10, 479-499. doi: 10.2147/PPA. S93046

8. O’Leary, S. T., Cataldi, J., Lindley, M. C., Beaty, B. L., Hurley, L. P., Crane, L. A., Brtnikova, M., Gorman, C., Vogt, T., Kang, Y., \& Kempe, A. US Primary Care Providers' Experiences and Practices Related to Routine Pediatric Vaccination During the COVID-19 Pandemic. Centers for Disease Control and Prevention. Published March 23, 2021. Accessed May 9, 2021. https://www. cdc.gov/vaccines/hcp/pediatric-practices-during-COVID-19. html

Financial Disclosures: None reported.

Support: None reported.

Ethical Approval: Touro University California IRB: Application Number M-1820

Status: Exempt

Informed Consent: Informed consent was obtained by providing a written invitation to participation for each social media post or email that was sent to potential participants. The beginning of the survey included a similar written explanation of the research project and information collected.

Poster No.: C8

Abstract No.: 33

Category: Clinical

Research Focus Area: Osteopathic Philosophy

\section{Understanding medical student perspectives in virtual osteopathic manipulative medicine education during the COVID pandemic}

\footnotetext{
${ }^{1}$ Thomas Liu, MS, DO; ${ }^{2}$ Martin Torrents, DO; ${ }^{3}$ Sahil Sharma, DO, PGY-II; ${ }^{2}$ Dennis Burke, DO; ${ }^{2}$ Dr. Athina Giovanis, DO

${ }^{1}$ Touro College of Osteopathic Medicine-Middletown (TouroCOM); ${ }^{2}$ Department of Osteopathic Manipulative Medicine, Touro College of Osteopathic Medicine - Middletown; ${ }^{3}$ Department of Emergency Medicine, Garnet Health Medical Center
}

Context: During the pandemic, medical education has been interrupted. Schools have been forced to shift toward virtual learning. In the past, pre-recorded videos have been recommended as a supplement to in-person lab education. To our knowledge, full virtual learning OMM lab has never been attempted. Our goal with this study was to evaluate the student's perspectives about our virtual OMM lab course. We hypothesize the results would show a negative response due to the lack of hands-on practice.

Objective: To assess osteopathic medical student's perspectives regarding virtual learning with osteopathic manipulative medicine (OMM) versus traditional inclassroom learning.

Methods: The survey study consisted of a ten-question survey using a Likert scale design. The study was designed with Microsoft Forms and an email request was sent to all 1st and 2nd-year medical students at TCOM asking for completion of the electronic survey. The questions focused on student's perspectives on confidence in learning, academic preparedness, and comparison of the aspects of the virtual and in-class learning settings. Data collection had no identifying information and responses were anonymous. No compensation was provided for student participation. IRB approval was obtained for this study.

Results: A total of 180 responses from 239 students were obtained with a response rate of $75.3 \%$. $68 \%$ of the responses did not generally enjoy the virtual learning environment. $63 \%$ of the responses did not feel that OMM was taught more efficiently through virtual learning. $71 \%$ of the responses did not feel confident overall. Free text response was allowed for the last survey question. Some comments suggested that virtual learning allowed a better understanding of the material especially with the use of small group "breakout” sessions. Other comments suggested the technology involved with virtual learning allowed for better communication among peers.

Conclusion: Responses of the survey study appear to support our hypothesis regarding a negative perspective to virtual learning due to the lack of hands-on practice. However, some responses suggest that virtual learning may be helpful as a supplement to traditional in-class OMM education. More collaboration among our peers in the OMM may help elucidate what is the most efficient manner in using virtual education for the future. 


\section{References}

1. Ferrel MN, Ryan JJ. The Impact of COVID-19 on Medical Education. Cureus. 2020;12(3):e7492. Published 2020 Mar 31. doi:10.7759/ cureus.7492

2. Sahi PK, Mishra D, Singh T. Medical Education Amid the COVID-19 Pandemic. Indian Pediatr. 2020;57(7): 652-657. doi:10.1007/ s13312-020-1894-7

3. Ray AM, Cohen JE, Buser BR. Osteopathic emergency physician training and use of osteopathic manipulative treatment. J Am Osteopath Assoc. 2004;104(1):15-21.

4. Snider KT, Seffinger MA, Ferrill HP, Gish EE. Trainer-to-student ratios for teaching psychomotor skills in health care fields, as applied to osteopathic manipulative medicine [published correction appears in J Am Osteopath Assoc. 2012 Jun;112(6):385]. J Am Osteopath Assoc. 2012;112(4):182-187.

5. Seals R, Gustowski SM, Kominski C, Li F. Does Replacing Live Demonstration With Instructional Videos Improve Student Satisfaction and Osteopathic Manipulative Treatment Examination Performance?. J Am Osteopath Assoc. 2016;116(11):726-734. doi:10.7556/jaoa.2016.143

Financial Disclosures: None reported.

Support: None report.

Ethical Approval: Institutional Review Board approved exempt - \#HSIRB_2048.

Informed Consent: Not applicable.

Poster No.: *C9

Abstract No.: 34

Category: Clinical

Research Focus Area: Chronic Diseases \& Conditions

\section{Long Term Subjective Functional Outcomes of Prostate Cancer: Prostatectomy VS. Radiation}

${ }^{1}$ Kellie Gaura OMS-III; ${ }^{1}$ Bryce Beatty, OMS-III; ${ }^{1}$ Natalie Ohlde, OMS-II; ${ }^{2}$ Elisabeth Guenther, MD; ${ }^{3}$ Johannie Spaan, $\mathrm{PhD}$

${ }^{1}$ Western University of Health Sciences College of Osteopathic Medicine of the Pacific (WesternU/COMP); ${ }^{2}$ Department of Career and Professional Development, Western University of Health Sciences College of Osteopathic Medicine of the Pacific (WesternU/COMP); ${ }^{3}$ Department of Research, Western University of Health Sciences College of Osteopathic Medicine of the Pacific (WesternU/COMP)
Context: Prostate cancer is the one of the most common cancers in men with over 190,000 men diagnosed annually. ${ }^{1}$ Prostate cancer is well-researched from an objective point of view in terms of treatment and prognosis, but the long term subjective outcomes from a patient's perspective remains relatively unknown. ${ }^{2}$ The Oregon Urology Institute (OUI) has been collecting subjective functional outcome data on patients since 2008.

Objective: To determine the long term subjective outcomes of prostatectomy versus radiation therapy and determine if the functional outcome scores can predict a patient's satisfaction with their treatment. This study aims to address the gap in research and patient reported outcomes in prostate cancer.

Methods: Patients treated at OUI were asked to complete a mailed Expanded Prostate Index Composite (EPIC) questionnaire post-treatment at regular intervals. The survey assessed various functional outcomes on a subjective basis such as: urinary function, bowel function, sexual function, hormonal function, and overall satisfaction of treatment. Patients were divided into two cohorts, ones treated surgically via prostatectomy vs radiation. Each cohort completed this survey post-treatment at 3, 6, 9, and 12 months then annually for the past 12 years (radiation patients) and 14 years (surgical patients). Results from each cohort were compared and analyzed with a linear mixed effect model.

Results: Both radiation and surgery cohorts consisted of 824 patients each. Urinary function score decreased by $4 \%$ for every month post treatment with radiation $(\mathrm{P}<0.0001)$, whereas surgery had a $5 \%$ increased urinary function score. Sexual functional scores after radiation decreases by $8 \%$ for every month post treatment $(\mathrm{P}<0.0001)$, whereas surgery cohort had a 13\% increased sexual functional score $(\mathrm{P}<0.0001)$. Hormonal function score after radiation treatment increases by $3 \%$ for every month post treatment $(\mathrm{P}<0.0001)$, whereas surgery cohort had a $2 \%$ decreased hormonal functional score $(\mathrm{P}=0.0001)$. Satisfaction scores for overall treatment after radiation treatment decreased by $3 \%$ for every month post treatment $(\mathrm{P}<0.0001)$, whereas surgery patients have a $2 \%$ higher satisfaction score $(\mathrm{P}=0.0065)$. The odds of a prostate cancer patient's satisfaction score increased by $7 \%$ for every one unit increase in urinary function and by $17 \%$ for every one unit increase in patients hormonal functional scores, after accounting for treatment $(\mathrm{P}<0.0001)$. 
Conclusion: There is a significant increase in overall subjective function and satisfaction of treatment with patients treated surgically vs radiation for prostate cancer.

\section{References}

1. Survival rates for prostate cancer. The American Cancer Society. Updated February 2, 2021. Accessed February 26, 2021. https:// www.cancer.org/cancer/prostate-cancer/detection-diagnosisstaging/survival-rates.html

2. Sanda MG, Dunn RL, Michalski J, et al. Quality of life and satisfaction with outcome among prostate-cancer survivors. New England Journal of Medicine. 2008;358(12):1250-1261. Accessed December 17, 2021. doi:10.1056/NEJMoa074311. https://pubmed. ncbi.nlm.nih.gov/18354103/

Financial Disclosures: None reported.

Support: None reported.

Ethical Approval: IRB \#: 21/RFD/005. The project was deemed to be not human research and was approved but the IRB at Western University of Health Sciences.

Informed Consent: The subjects of this study are all patients at the Oregon Urology Institute (OUI). At the clinic they were given written consent forms that they signed before filling out the functional outcomes survey. All data was collected by OUI and then retrospectively examined by the research team.

Poster No.: ${ }^{\star} \mathrm{C} 10$

Abstract No.: 35

Category: Clinical

Research Focus Area: Impact of OMM \& OMT

\section{The Efficacy of an OMM Virtual Reality Program on Learning for Osteopathic Medical Students}

${ }^{1}$ Jerry Jose OMS-III; ${ }^{2}$ Erum Ahmed, OMS-IV; ${ }^{2}$ Edward Piscitelli, OMS-III; ${ }^{2}$ Randy F. Stout, PhD; ${ }^{3}$ Sheldon C. Yao, DO, FAAO

${ }^{1}$ New York Institute of Technology (NYITCOM); ${ }^{2}$ Department of Biomedical Sciences, New York Institute of Technology (NYITCOM); ${ }^{3}$ Department of Osteopathic Manipulative Medicine, New York Institute of Technology (NYITCOM)

Context: Virtual Reality (VR) is a relatively new medium created to provide the public with an immersive and interactive gaming experience. In recent years, VR has made its way into medical education, gamifying the learning environment (1). Studies have shown the benefits of using VR for surgical training and anatomy learning ${ }^{(1,2)}$; however, this novel approach has not been explored in the field of osteopathic medicine. So, we set out to create a VR OMM program that would enhance osteopathic learning.

Objective: To determine whether the use of Virtual Reality (VR) for learning Osteopathic Manipulative Medicine (OMM) results in high learning motivation among osteopathic medical students. We believe that the unique aspects of medical VR that allow for the interactive viewing of the human body with immediate feedback, will motivate students to learn and accurately practice osteopathic diagnoses and techniques.

Methods: A pilot VR OMM program was developed to determine if osteopathic medical students felt that the use of VR would enhance the learning of osteopathic techniques and diagnosis. The program was designed and developed using Unity development software. A virtual reality OMM classroom environment was created for students to enter using an Oculus VR Headset and practice their osteopathic techniques such as identifying landmarks, assessing symmetry, determining thoracic range of motion, and conducting the skin drag and erythema tests. An email regarding the pilot study was sent out to first year medical students at NYITCOM and those who volunteered were lent an Oculus VR Headset for one week with video instructions on how to use the program. Total play times and scores during each game session were recorded using the headset, and Instruction Materials Motivation (IMMS) and OMM in VR feedback surveys were given after headset use to assess the students' thoughts and motivation to learn OMM using a VR medium ${ }^{(3,4)}$. The IMMS survey assessed the students' motivation through four categories: attention, relevance, confidence, and satisfaction (ARCS) (5). From the IMMS survey, which consisted of 36 questions, the 12 Reduced Instructional Material Motivation Survey (RIMMS) questions were used for analysis (3). The RIMMS contains three questions pertaining to each category of the ARCS Model of Motivation. The surveys, except the free response section, were answered on a 1-5 Likert-scale. SPSS was used to statistically analyze the data and check for overall agreement or disagreement on the practicality and benefits of using VR for OMM.

Results: A total of 45 first year osteopathic medical students attending NYITCOM expressed interest in being a part of this pilot study, out of which 44 students actively participated in the OMM VR program. From these $44 \mathrm{stu}-$ dents, 39 filled out the post-program survey, however only 37 fully completed the survey to its entirety. From the 44 
students who participated in the program, the data collected from the headsets showed students spent an average of 11.5 minutes in the OMM VR program, with the average first score being 68.3 and the average highest score being 75.5 . The modes, medians, and interquartile ranges (IQR) of the 1-5 Likert-scale responses were calculated for each question in the RIMMS. The results of the statistical analysis were as follows: attention (modes $=3,4,4$; medians $=3$, 4, 4; IQR $=1,1,1$ ), relevance (modes $=5,3$, 4; medians $=5$, 4, 4; IQR $=1,1,1)$, confidence (modes $=5,5$, 4; medians $=4$, 4, 4; IQR=2, 2, 1), and satisfaction (modes $=3$, 5, 5; medians=4, 4, 4; IQR=2, 2, 2). The modes and medians show there was an overall agreement that practicing OMM in the VR program was useful and motivating. The IQR ranges of 1 and 2 also show that there was little disagreement between the students for each question. Student comments in the free response section confirmed the overall positive experience with OMM and VR. Students liked how it allowed them to review and practice skills they already learned in class, and they expressed interest in seeing more OMM techniques as modules in the future. Additional survey responses pointed out that there were some challenges to learning how to use the VR controls and that improved instructions for the module could be helpful for the future.

Conclusion: This was a survey-based pilot study that aimed to determine whether OMM could be brought to VR in a meaningful way. The goal was to determine if practicing OMM in VR would be helpful for students, and if it could motivate them as a novel additional learning tool. The results of the RIMMS and OMM in VR survey showed that OMM could potentially be brought into VR for remote or even in class learning. We also received useful feedback on what worked and what could be improved for the next phase of this study. The next version of this program will include more techniques and will incorporate some of the suggested improvements to the pilot program. The use of virtual reality could pave the way for the future use of outof-classroom OMM learning and assessment to fine tune osteopathic skills and create enthused and motivated osteopathic physicians.

\section{References}

1. Nicola S, Virag I, Stoicu-Tivadar L. VR medical gamification for training and education. Health Informatics Meets eHealth. 2017:97-103. doi:10.3233/978-1-61499-759-7-97

2. Samadbeik M, Yaaghobi D, Bastani P, Abhari S, Rezaee R, Garavand $A$. The applications of virtual reality technology in medical groups teaching. Journal of Advanced Medical Education and Professionalism. 2018;6(3):123-129.

3. Loorbach N, Peters O, Karreman J, Steehouder M. Validation of the Instructional Materials Motivation Survey (IMMS) in a self-directed instructional setting aimed at working with technology. British Journal of Educational Technology. 2014;46(1):204-218. doi:10. 1111/bjet.12138

4. Sattar MU, Palaniappan S, Lokman A, Hassan A, Shah N, Riaz Z. Effects of Virtual Reality training on medical students' learning motivation and competency. Pakistan Journal of Medical Sciences. 2019;35(3). doi:10.12669/pjms.35.3.44

5. Reigeluth CM, Keller JM. Motivational design of instruction. In: Instructional design theories and models: An overview of their current Status. Hillsdale, New Jersey: Lawrence Erlbaum Associates; 1983:386-434.

Financial Disclosures: None reported.

Support: None reported.

Ethical Approval: This study was approved by the NYIT Institutional Review Board - BHS-1582.

Informed Consent: Research subjects were informed of the purpose, description, potential risks and potential benefits of the research study by a member of the research team. Research subjects then signed an informed consent form after being given adequate time to ask any questions they may have. All health information of research subjects remained confidential and accessed only by members of the research team. Research subjects voluntarily agreed to participate in this study and were given the option to withdraw from the study at any time without penalty or loss of benefits.

Poster No.: ${ }^{\star} \mathrm{C} 11$

Abstract No.: 42

Category: Clinical

Research Focus Area: Chronic Diseases \& Conditions

\section{At-Home ECG Monitoring with a Real-Time Outpatient Cardiac Telemetry System During the COVID-19 Pandemic}

${ }^{1}$ Nolberto Jaramillo, Jr., OMS-II; ${ }^{2}$ Denis Malkov, OMS-II; ${ }^{2}$ Uddampreet Singh Arora, OMS-II; ${ }^{2}$ Jacqueline Nikakis, OMS-II; ${ }^{2}$ Todd J. Cohen, MD

${ }^{1}$ New York Institute of Technology (NYITCOM); ${ }^{2}$ Department of Clinical Specialties, New York Institute of Technology (NYITCOM) 
Context: During the COVID-19 pandemic, hospitals closed catheterization and electrophysiology labs while many patients continued to suffer from cardiac conditions. An emergency declaration expanded telehealth utilization, however, essential in-person ECG recordings became more difficult. To circumvent these challenges, the cardiology clinic at NYIT College of Osteopathic Medicine (NYITCOM) transitioned to remote real-time outpatient cardiac telemetry (ROCT) using devices shipped to patients' homes.

Objective: To test the hypothesis that at-home ROCT, provided by the Cardiac Clinic at NYITCOM, is an effective method of providing ECG monitoring to symptomatic patients during the COVID-19 pandemic.

Methods: "Diagnostic and Therapeutic Outcomes from the Long Island Heart Rhythm Center (LIHRC)”, is an NYITCOM Institutional Review Board approved registry that permits this retrospective analysis of observations and outcomes of cardiac patients (BHS-1464). All patients who received athome ROCT care by the clinic's team during the first COVID-19 surge were included in the analysis (from 3/11/ 2020-8/1/2020). The FDA-approved ROCT devices (DMS-300 from Stateline, Nevada) were shipped to and from the patients' homes and the electrodes were selfapplied to patients' chests through adhesive patches. These small, waterproof, plastic devices were connected to a computer at the clinic's base which stores real-time data using cloud-based Smartsheet ${ }^{\circledR}$ software. The system then produced clinical reports which included a continuous 6-lead ECG and many cardiovascular health parameters including heart rate, heart rate variability, ST-segment analysis, supraventricular and ventricular ectopy, bradycardia (including significant pauses $>2.5 \mathrm{~s}$ ), QT/QTc intervals, as well as other arrhythmias. Additionally, patients were instructed to activate the device's symptom recorder to correlate symptomatic episodes to the ECG recordings. The patients' medical records were de-identified and reviewed for age, gender, indications, and findings. Patient comfort and ease of use of the devices were important aspects evaluated in consideration of the mind and spirit in the osteopathic approach and the psycho-behavioral components of osteopathic care. The data was analyzed and presented as the mean \pm standard deviation.

Results: 17 patients (15 female) from the LIHRC were included in the analysis with an average monitoring duration of 27 hours. The patients' ages ranged from 21 to 85 years old with a mean of $37 \pm 19$ years old. ROCT indications included palpitations $(n=8)$, presyncope $(n=9)$, chest pain $(n=5)$, shortness of breath $(n=2)$, and syncope $(n=3)$. Some also received ROCT due to short PR intervals observed on a pre-pandemic ECG $(n=3)$.

Two patients experienced palpitations while wearing the ROCT device: one had supraventricular tachycardia at 150 beats per minute; the other had unifocal premature ventricular contractions, and eventually underwent a successful cardiac ablation. Most patients experienced no symptomatic episodes during ROCT $(n=15)$. The 6-lead ECG for five of those patients showed arrhythmias including wandering atrial pacemaker $(n=2)$, sinus tachycardia $(n=1)$, premature ventricular contractions $(\mathrm{n}=1)$, premature atrial and ventricular contractions ( $n=1)$, ectopic atrial rhythms $(n=1)$, and sinus arrhythmia $(n=1)$. The LIHRC staff experienced some device/connectivity issues. The majority of patients had no problems, however four patients had the following issues: two removed their devices due to discomfort, one could not set up the device, and one could not use the device due to poor internet connection.

Conclusion: This innovative remote real-time cardiac telemetry service circumvented the challenges presented by the COVID-19 lockdown by allowing ECGs to be performed at-home. ROCT also produced an ECG recording for a longer duration than the typical in-person 12-lead ECG, which occurs at a single time point. In addition, the system successfully correlated the patients' cardiac arrhythmia symptoms to ECG findings. The ROCT device provided 24/7 6-lead ECG monitoring and various other cardiac health parameters. Most patients and staff were able to use the system without issues.

With the unique challenges of the COVID-19 pandemic, physicians can use at-home ROCT to prevent infection and diagnose cardiac conditions. This can also be used to deliver patient-centered care to those with limited mobility when coupled with a telemedicine visit.

This study is limited by its retrospective and observational nature, and the small sample size. Further studies are necessary to determine the effectiveness of at-home ROCT ECG monitoring compared to the traditional inperson ECG.

References Not applicable.

Financial Disclosures: None reported.

Support: None reported.

Ethical Approval: The IRB approval process determined the study to be exempt status due to its de-identified, retrospective nature (BHS-1464).

Informed Consent: Not applicable.

Poster No.: ${ }^{\star} \mathrm{C} 12$

Abstract No.: 46

Category: Clinical 
Research Focus Area: Osteopathic Philosophy

\section{Examining Harassment Based on Gender Identity in Online Gaming Platforms}

${ }^{1}$ Nicole Volino, OMS-III; ${ }^{2}$ Joanne DiFrancisco-Donoghue, MS, PhD; ${ }^{2}$ Sophia Ahmad, OMS-IV

${ }^{1}$ New York Institute of Technology (NYITCOM); ${ }^{2}$ Department of Osteopathic Manipulative Medicine, New York Institute of Technology (NYITCOM)

Context: There are more than 214 million video game players in the United States today, with $41 \%$ of those being women. ${ }^{1}$ Research has shown that women are experiencing harassment while gaming online, which can lead to anxiety and stress. ${ }^{2}$ Cyber harassment has been shown to have a detrimental effect on mental health, including depression, anxiety, suicidal ideation, and panic attacks. ${ }^{3}$ The mental health impact that online harassment may have is of concern for physicians who treat women gamers.

Objective: The primary purpose of this study was to examine the harassment that women who play video games are experiencing while gaming. This included harassment directed at their gender identity, sexual harassment, and non-sexual harassment. Secondarily, this survey examined how this harassment impacted their willingness to play video games and how they felt gaming platforms addressed the harassment.

Methods: Data for this study were collected utilizing an online survey. This survey was exempt by the Institutional Review Board at New York Institute of Technology. The survey consisted of 18 multiple choice and open ended questions. There were no personal identifiers used according to HIPAA and NIH guidelines, therefore consent was not required. The survey was distributed to online gaming platforms that included Twitch, Discord, Twitter, Reddit, and Facebook. Descriptive statistics were used to analyze and describe all data. Many physicians may not be aware that the cyber harassment women face can impact both mental and physical health. The results from this study will help bring awareness to osteopathic physicians, who can then help these women achieve optimal health by addressing the needs of both their mind and body.

Results: There were 166 survey respondents. Because this study is focused on women gamers, 22 respondents who listed their gender identity as male were excluded. Of the 144 remaining participants, $19.4 \%$ (28) were ages $18-20$, $59.0 \%$ (85) were ages $21-30,16.0 \%$ (23) were ages $31-40$, and 5.6\% (8) were older than 40 years. The results indicated that $82.6 \%$ (119) of respondents had reported experiencing at least one form of harassment while gaming, $68.1 \%$ (98) reported experiencing harassment based on their gender identity, 59.0\% (85) reported experiencing sexual harassment, and $72.7 \%$ (104) reported experiencing non-sexual harassment. When asked if sexual harassment is properly addressed in the gaming community, only $7.6 \%$ (11) responded "yes", while $32.0 \%$ (46) responded "sometimes" and $60.4 \%(87)$ responded "no". When asked the same question of non-sexual harassment, $7.7 \%$ (11) responded "yes". 33.6\% (48) responded "sometimes", and $58.7 \%$ (84) responded "no". Lastly, when asked if harassment had impacted their willingness to play games, 56.5\% (48) of those who had experienced sexual assault responded "yes", while $58.7 \%$ (61) of those who had experienced non-sexual assault responded "yes". At the end of the survey, participants were asked to describe a time they had experienced harassment based on their gender identity, if they felt comfortable enough to do so. Of those that answered, several respondents mentioned being told to "get back in the kitchen" (12), hearing sexually explicit comments (16), being asked for sexually explicit photos (3), and hearing comments pertaining to or threatening rape (6).

Conclusion: The results of this survey suggest that over $80 \%$ of women gamers are experiencing harassment while playing online video games. Of those, more than half indicated that the harassment has impacted their willingness to play games. Furthermore, most of the respondents did not feel as though the gaming community is doing enough to address both non-sexual and sexual harassment. Some limitations of this study include the number of respondents and that the level of harassment that men who game are experiencing was not collected and compared. Future studies should be done in a larger cohort and should include all genders. Cyber harassment, coupled with a lack of responsiveness from online platforms, can have serious, detrimental effects on women's mental health. Although mental health is multi-factorial, it's important for physicians to be aware of their patients who game and the possible harassment that may be contributing negatively to their physical and mental health.

\section{References}

1. Fitzgerald D. 2020 Essential Facts About the Video Game Industry. Entertainment Software Association. Accessed July 9, 2021. https://www.theesa.com/resource/2020-essential-facts/ 
2. McLean L., Griffiths M.D. Female Gamers' Experience of Online Harassment and Social Support in Online Gaming: A Qualitative Study. Int J Ment Health Addiction 17, 970-994 (2019). https://doi. org/10.1007/s11469-018-9962-0

3. Stevens F., Nurse J., Arief B. Cyberpsychology, Behavior, and Social Networking.Jun 2021.367-376. https://doi.org/10.1089/ cyber.2020.0253

Financial Disclosures: None reported.

Support: None reported.

Ethical Approval: This study was deemed exempt by the Institutional Review Board at New York Institute of Technology. BHS-1547.

Informed Consent: There were no personal identifiers used according to HIPAA and NIH guidelines, therefore consent was not required.

${ }^{\star}$ Poster No.: ${ }^{\star} \mathrm{C} 13$

Abstract No.: 48

Category: Clinical

Research Focus Area: Impact of OMM \& OMT

\section{Assessing usage and perceptions of osteopathic manipulative treatment (OMT) and self-identity among osteopathic physicians}

${ }^{1}$ Mahima Mangla, OMS-III; ${ }^{2}$ Saljooq Asif, MS, OMS-II; ${ }^{2}$ Soubhana Asif MA, OMS II; ${ }^{2}$ Michael J. Terzella, DO; ${ }^{2}$ Sheldon Yao, DO

${ }^{1}$ New York Institute of Technology (NYITCOM); ${ }^{2}$ Department of Osteopathic Manipulative Medicine, New York Institute of Technology (NYITCOM)

Context: Understanding viewpoints towards OMT and degree of OMT usage can identify factors influencing its practice. ${ }^{1,2}$ Given the recent single accreditation, exploring how osteopathic physicians (D.O.) believe their education sets them apart can highlight what aspects of osteopathic training should be maintained..$^{3-5}$ The notion of stigma against D.O.s will also be investigated. In doing so, we can establish how to support positive D.O. self-identity and even societal perceptions of OMT.

Objective: To explore the degree to which D.O.s were exposed to OMT in training and currently practice OMT. We also seek to understand barriers and facilitators to the use of OMT. Finally, we examine how receiving osteopathic training might influence clinical practice, future opportunities, and growth as medical professionals.

Methods: All members of the New York State Osteopathic Medical Society (NYSOM; N=300) were surveyed on their use of OMT during training and in present clinical practice. The survey collected demographic information, perceptions of the osteopathic profession, thoughts on the future direction of osteopathic medicine, and anecdotal data on how being a D.O. might have influenced career advancement. The survey was sent to all members via email using REDCap. Follow-up efforts included weekly emailed reminders for one month from the first send date in June 2021. Statistical analyses were performed to establish factors influencing use of OMT during training and practice.

Results: In total, $22.0 \%$ (65/300) of distributed surveys were completed during the recruitment period. Of the sample, 49.0\% (32/65) practice primary care (family medicine, internal medicine, pediatrics, or geriatrics), $75.0 \%$ (49/65) have been in practice for over 10 years, and $71 \%$ (46/65) completed an osteopathic residency. 80.0\% (49/61) of respondents reported that osteopathic training gave them an advantage over allopathic counterparts. Next, $57.0 \%$ (37/65) reported observing OMT and 78.0\% (47/60) reported utilizing OMT during training. At present, 48.0\% $(30 / 62)$ of respondents practice OMT. When assessing barriers to the practice of OMT, $49.0 \%$ (27/55) of respondents stated time, 33.0\% (18/55) indicated reimbursement, and 25.0\% (14/55) noted confidence level in OMT skills as important reasons preventing use of OMT. Other barriers include patient population, physical space, institutional support, and patient and colleague skepticism about OMT. 54.0\% (32/65) of respondents also shared that their allopathic medical colleagues have expressed interest in osteopathic principles and techniques. $88 \%$ of respondents agreed or strongly agreed to both statements that that OMT positively enhances the doctor-patient relationship and yields positive effects on patient health outcomes. When asked about stigma, 41\% (28/65) reported discrimination due to training as an osteopathic physician. Analysis of qualitative responses highlights missed professional, teaching, and training opportunities due to respondents' osteopathic medical degrees. Finally, while $46.0 \%$ of respondents agreed or strongly agreed that there is a stigma associated with osteopathic medicine in the medical field, $31.0 \%(14 / 45)$ disagreed or strongly disagreed with this statement. 
Conclusion: Observation and utilization of OMT were greater during training then in clinical practice postresidency. Barriers to OMT that were most influential included time, reimbursement, and physician confidence in OMT skills. Most respondents indicated that they found OMT helpful for patients and noted that it built a therapeutic alliance between patient and provider. Unfortunately, many physicians noted stigma associated with their medical training. Lack of professional recognition, fewer hospital privileges, fewer residency sites, and lost teaching opportunities comprised many of the stated reasons. It is important to note some limitations of this study. First, there could be a regional bias as the survey sample was only from New York. Also our overall sample size is small so our claims might not extend reliably to a larger sample of osteopathic physicians. As the osteopathic profession evolves, gleaning viewpoints towards OMT and osteopathic education from D.O.s can help shape future efforts to support positive D.O. identity and maintain what distinguishes the profession.

\section{References}

1. Healy CJ, Brockway MD, Wilde BB. Osteopathic manipulative treatment (OMT) use among osteopathic physicians in the United States. Journal of Osteopathic Medicine. 2021;121(1):57-61. doi:10. 1515/jom-2020-0013

2. Barnhardt EW, Comer F, Zmuda E, Rakowsky A. Impact of an osteopathic presence in a large categorical pediatric residency training program. Journal of Osteopathic Medicine. 2021;121(1):35-42. doi:10.1515/jom-2019-0317

3. Benefits of Single GME. Accreditation Council for Graduate Medical Education (ACGME). https://www.acgme.org/What-WeDo/Accreditation/Single-GME-Accreditation-System/Benefitsof-Single-GME

4. Connett DA. Effect of the Single Accreditation System. Journal of Osteopathic Medicine. 2014;114(7):524-526. doi:10.7556/jaoa. 2014.101.

5. Gevitz N. Center or Periphery? The Future of Osteopathic Principles and Practices. Journal of Osteopathic Medicine. 2006;106(3):121129. doi.org/10.7556/jaoa.2006.106.3.121

Financial Disclosures: None reported.

Support: None reported.

Ethical Approval: The NYITCOM IRB reviewed and approved the IRB for this survey study. The IRB number of this study is BHS1648 and it was approved on 5-10-21.

Informed Consent: Consent was implied by completion of the optional survey.

Poster No.: C14

Abstract No.: 49

Category: Clinical
Research Focus Area: Osteopathic Philosophy

\section{Ultrasound Examinations of the} Head and Neck Anatomy Increases Student's Confidence of Anatomical and Clinical Comprehension of a Complex Region

${ }^{1}$ Randall L. Nydam, PhD; ${ }^{1}$ Jay F. Olson, DO; ${ }^{1}$ Amanda G. Mark, DO; ${ }^{1}$ Victoria J. Smith, DO; ${ }^{1}$ Danielle K. Garner, DO; ${ }^{1}$ Samantha L. Rudy, OMS-V; ${ }^{2}$ Charles A. Finch, Jr, DO FACOEP; ${ }^{3}$ Randall L. Nydam, PhD

${ }^{1}$ Midwestern University Arizona College of Osteopathic Medicine (MWU/AZCOM); ${ }^{2}$ Department of Integrated Medicine, Midwestern University Arizona College of Osteopathic Medicine (MWU/AZCOM); ${ }^{3}$ Office of the Dean, Midwestern University Arizona College of Osteopathic Medicine (MWU/AZCOM)

Context: With the increased utilization of ultrasound in the clinical setting today, ultrasound education has rapidly become integrated in the curricula of medical schools to assist students in gaining a more holistic understanding of anatomy. This integration was to enforce and supplement knowledge of anatomical systems and relationships. The head and neck are sites for numerous clinical ultrasound procedures, thus head and neck ultrasound workshops are an essential component in clinical education.

Objective: To evaluate the effectiveness of two head and neck ultrasound workshops toward a student's understanding of this region's complex anatomy.

Methods: Two ultrasound workshops were developed to provide students an opportunity to explore the soft tissue encompassing the orbit-an examination to assess the eye as well as offer a method to assess increased intracranial pressure - and the anterior triangle of the neck which includes the carotid artery-commonly scanned using ultrasound to measure stenosis. Both workshops were developed with the assistance of skilled physicians and sonographers.

After participating in head- and neck-specific ultrasound workshops, all AZCOM and AZPOD students from the 2017-2018 and 2018-2019 academic year were emailed an anonymous survey to gauge their perception of the utilization of ultrasound examinations. The surveys were also evaluated on how the workshops aided their holistic understanding of the relatability of structure and function in the head and neck anatomy during the gross anatomy courses in 2017-2018 and 2018-2019 academic years. After 
the collection period, the Likert-style questions were converted to numerical data on a scale of 1 to 5 with 1 being "strongly agree", 3 being "neither agree nor disagree", and 5 being "strongly disagree". The data was then analyzed with independent t-tests using SPSS software to determine if there were differences in perception between those in the different academic programs or between identified genders.

Results: The response rate for the survey that was distributed after the workshop on the orbit for both the 2017-2018 as well as 2018-2019 academic years were 66\%. In general, the respondents overwhelmingly "strongly agreed" that the ultrasound integration enhanced their anatomical studies (Likert-style quantification of 1.79 with a standard deviation (SD) of 0.88), prepared them for future clinical experiences (1.68, SD 0.81), as well as helped them interpret ultrasounds more confidently (1.59, SD 0.80). Most students agreed that the ultrasound workshop had inspired them to do further research on the clinical concerns for the eye (2.41, SD 1.07). There were no significant differences ( $p>0.05$ ) on any of the questions between the academic programs or genders.

The response rate for the survey for both academic years after the anterior neck workshop was $29 \%$. In general, the respondents overwhelmingly "strongly agreed" that the ultrasound integration enhanced their anatomical studies (1.57, SD 0.69), prepared them for future clinical experiences (1.52, SD 0.64), as well as helped them interpret ultrasounds more confidently (1.41, SD 0.65). Most students agreed that the ultrasound workshop had inspired them to do further research on the clinical concerns for the eye (2.30, SD 1.00). There were no significant differences ( $p>0.05$ ) on any of the questions between the academic programs. Female students more strongly agreed that the carotid artery assessment workshop led them to further clinical investigation on their own than male students (1.94, SD 0.83 vs 2.50 SD 1.06; $p=0.006$ ).

Conclusion: The integration of ultrasound in the head and neck gross anatomy curriculum was greatly supported by both genders of osteopathic and podiatric medical students as a supplemental tool to strengthen their understanding of the complex nature of regional anatomy and its clinical correlations. These students felt that utilizing this technology while exploring this region will be useful in their future practice in the clinical settings. This study was conducted collecting subjective data via survey results and was limited to a single medical school, paving the way for further investigation into whether ultrasound workshop integration in gross anatomy is correlated with improved test scores. Overall, this study provides significant evidence that ultrasound workshops can be integrated with the gross anatomy courses in medical schools to provide students with a more holistic understanding of the head and neck.

\section{References None}

Financial Disclosures: None reported.

Support: Funding from MWU AZCOM and a grant from HonorHealth Phoenix General Hospital Osteopathic Education Foundation training and curricular development assistance contributed to help establish the ultrasound integration at Midwestern University Arizona College of Osteopathic Medicine.

Ethical Approval: This study was given Institutional Review Board approval from the IRB at Midwestern University in October 2017, IRB number 1092

Informed Consent: Informed Consent statement was included in the survey that was emailed to the students of the 2017-2018 and 2018-2019 Arizona College of Osteopathic Medicine and Arizona School of Podiatric Medicine

Poster No.: ${ }^{\star} \mathrm{C} 15$

Abstract No.: 51

Category: Clinical

Research Focus Area: Osteopathic Philosophy

Factors Affecting Osteopathic Medical Students' Specialty Choice in Light of a Future Pass/Fail COMLEX Level 1 and USMLE Step 1

${ }^{1}$ Risa Nicole Kiernan, OMS-III; ${ }^{2}$ Katherine Keever, OMS-III; ${ }^{2}$ Ashley Monaco, OMS-III; ${ }^{2}$ Maria Pino, PhD, MS, Rph; ${ }^{2}$ Gregory Saggio, DO

${ }^{1}$ New York Institute of Technology (NYITCOM); ${ }^{2}$ Department of Clinical Specialties, New New York Institute of Technology (NYITCOM)

Context: Osteopathic medical students (OMS) continue to pursue medical specialties outside of primary care. ${ }^{1,2}$ One factor crucial to OMS pursuing these fields is COMLEX Level 1/USMLE Step 1 scores. With changes to pass/fail grading of these exams, it is unknown how this may affect OMS. Studies have shown that factors such as medical school hospital affiliations, mentorship, and research will become increasingly important, potentially putting OMS interested in these fields at a disadvantage.,

Objective: To investigate differences in perceived barriers of OMS applying to residency between residents and current medical students before the new COMLEX Level 1 and USMLE Step 1 pass/fail grading format. Results from this study can serve as a tool for medical school administrators 
to better advise OMS when applying to residency in the future and aid in the development of a curriculum that enhances OMS interests.

Methods: This is a cross-sectional study of OMS from the classes of 2022 - 2024 and recent medical school graduates from New York Institute of Technology College of Osteopathic Medicine (NYIT-COM) from both the New York and Arkansas campuses. From March-June 2021, 1,525 OMS and recent graduates were asked to complete a survey regarding perceptions of factors that impact medical students' ability to match into their desired residency program. Following the initial email, three follow-up reminders were sent to all participants. The survey was administered electronically on Red Cap (Nashville) and institutional review board (IRB) approval was obtained from NYIT-COM. The survey consisted of 49 questions covering aspects of the residency application including residency interest, medical board exam results, clinical grades, mentorship, medical school reputation, clinical affiliations of the medical school, and research. Responses were rated on a 4-point Likert scale ranging from 1 ("strongly disagree") to 4 ("strongly agree"). Other variables such as demographics, board exam scores, and match year/anticipated match year were also collected. Respondents from the classes of 2022 - 2024 were labeled as "Prospective" and respondents from the classes of 2021 and earlier were labeled as "Retrospective" having already matched into a residency program. Statistical analyses were calculated using IBM SPSS, ver. 27 and $p$-values less than or equal to 0.05 were considered significant. Descriptive statistics for categorical variables are reported as absolute frequencies/percentages and were analyzed by a chi-square test. Continuous variables are reported as medians/interquartile ranges and are analyzed by a student's t-test. This study is important for the osteopathic medical community to gain a better understanding of factors that OMS perceive as being important to the residency match process and to allow medical schools to better cater curriculums towards OMS needs.

Results: A total of 221 completed surveys were received (15\% response rate) with $35 \%$ of responses being from the Prospective group and $65 \%$ of responses from the Retrospective group. Respondents were split almost evenly between male (49\%) and female (51\%) with the distribution of males and females in both the Prospective and Retrospective groups being nonsignificant. The majority of respondents were White $(61 \%)$, Asian $(25 \%)$, or Black $(5.0 \%)$ and 3.6\% were Hispanic or Latino. When comparing perceptions of various factors that influence OMS residency decisions between Prospective and Retrospective groups, both groups found USMLE Step 1/COMLEX Level 1 (82\% vs.
$84 \% ; \mathrm{p}=0.84)$ and USMLE Step 2/COMLEX Level 2 (78\% vs. $94 \% ; \mathrm{p}=0.20$ ) as being important factors in an individual's ability to match. Both groups agreed that pass/fail USMLE Step 1/COMLEX Level 1 would have put them at a disadvantage to match to their residency of choice ( $49 \%$ vs. $59 \%$; $\mathrm{p}=0.19$ ) with the majority of respondents agreeing that this change will make the medical school attended and its hospital affiliations more important when matching to a residency program ( $93 \%$ vs. $92 \% ; p=1.00)$. The majority of the Prospective group agreed their institution helped them find a mentor in their field of interest $(64 \%$ vs. $16 \%$ in Retrospective, $\mathrm{p}<0.05)$ and had a research mentor $(57 \%$ vs. $34 \%$ in Retrospective, $\mathrm{p}<0.05)$. The majority of the Prospective group also agreed that the number of publications on their residency application was important to match at their residency of choice, while less than half of the Retrospective group agreed (66\% vs. $41 \%$ in Retrospective, $\mathrm{p}<0.05)$. No significant differences were found between the Prospective and Retrospective groups perceiving boards scores, clinical grades, school affiliations, school reputation, Sigma Sigma Phi status, and clinical exposure as being important factors that influence students' decision to pursue a particular residency.

Conclusion: Our study confirms that in light of a pass/fail COMLEX Level1 and USMLE Step 1, there has been a shift in the factors that are perceived as important for residency by current and future OMS as compared to graduates. The most significant differences included the perceptions of research and mentorship, with more of the Prospective group agreeing these factors are important when deciding which residency to apply to. We speculate that this difference can be attributed to the recent change to a pass/fail COMLEX Level 1 and USMLE Step 1 with both groups agreeing board examination scores are an important component when choosing a residency and this change would have or will put them at a disadvantage with the residency match process. Because these scores will no longer be part of their decisionmaking process for choosing a residency, other factors such as COMLEX Level 2 and USMLE Step 2, which both groups agreed were important, as well as mentorship, research, and hospital affiliations will become increasingly important to OMS based on these preliminary results. This study had several limitations. First, it was administered to only one medical school and its branch campus and excluded the opinion of allopathic medical students. Second, this study did not include perceptions of residency directors. Future studies comparing both allopathic and OMS as well as opinions from residency directors should be evaluated to determine the implications of a pass/fail USMLE Step 1 and COMLEX Level 1. We believe this study may encourage osteopathic medical schools to establish formal mentorship 
programs for OMS and arrange for students to participate in research electives to improve their chance of securing their residency of choice.

\section{References}

1. Dogbey GY, Collins K, Russ R, Brannan GD, Mivsek M, Sewell S. Factors associated with osteopathic primary care residency choice decisions. J Am Osteopath Assoc. 2018;118(4):225-233. doi: 10. 7556/jaoa.2018.046

2. Cummings M. Osteopathic students' Graduate Medical Education aspirations versus realities: The relationship of osteopathic medicine and primary care. Acad Med. 2016;91(1):36-41. doi: 10. 1097/ACM.0000000000000892

3. Ehrlich $H$, Sutherland M, McKenney M, Elkbuli A. Implications of the United States Medical Licensing Examination Step 1 examination transition to pass/fail on medical students education and future career opportunities. Am Surg. Published online 2020:313482 0973382. doi: $10.1177 / 0003134820973382$

4. Goshtasbi K, Abouzari M, Tjoa T, Malekzadeh S, Bhandarkar ND. The effects of pass/fail USMLE Step 1 scoring on the otolaryngology residency application process. Laryngoscope. 2021;131(3): E738-E743. doi: 10.1002/lary.29072

Financial Disclosures: None reported.

Support: None reported.

Ethical Approval: Our study was reviewed and deemed exempt by the NYITCOM IRB committee. IRB number: BHS-1629

Informed Consent: Informed consent was not needed for this study.

Poster No.: ${ }^{\star} \mathrm{C} 16$

Abstract No.: 55

Category: Clinical

Research Focus Area: Osteopathic Philosophy

\section{Factors Influencing Osteopathic Medical Students' Decision to Pursue a Surgical Specialty}

\author{
${ }^{1}$ Katherine Keever, OMS-III; ${ }^{2}$ Risa Kiernan, OMS-III; ${ }^{2}$ Ashley \\ Monaco, OMS-III; ${ }^{2}$ Maria Pino, PhD; ${ }^{2}$ Gregory Saggio, DO \\ ${ }^{1}$ New York Institute of Technology (NYITCOM); ${ }^{2}$ Depart- \\ ment of Clinical Specialities, New York Institute of Tech- \\ nology (NYITCOM)
}

Context: Classically, the osteopathic medical profession has trained students towards primary care careers. Previous studies found clinical exposure as a key factor in osteopathic medical students (OMS) decision to focus on primary care. However, there are OMS who end up applying to surgical specialties. Studies on allopathic students have shown clerkships and mentorship as important for pursuing surgical specialties. It is unknown what factors are important to OMS pursuing surgical specialties.

Objective: To investigate perceived barriers of OMS applying to or already matched to surgical residency programs compared to those in non-surgical programs. These barriers are important to explore as while osteopathic medical schools classically train students to primary care careers, some OMS do apply to surgical specialties. This study can potentially serve as a tool to the medical school administration to better serve this cohort of OMS needs.

Methods: From March to June 2021, 1,525 students from an osteopathic medical school and its branch campus were emailed to fill out a questionnaire on their perceptions of what factors influence their ability to match into their residency choice. Participation was voluntary and the email was sent to third and fourth year OMS as well as graduates. The survey was administered electronically on the anonymous and secure web application RedCap. Three follow-up reminders were sent to the participants. Institutional review board (IRB) approval for this cross-sectional study was obtained from the New York Institute of Technology's IRB. The questionnaire consisted of 49 questions and covered aspects of the residency application based on a review of the literature. Questions included residency interest, boards scores, clinical grades, mentorship, hospital affiliations, and research. Responses were rated on a 4-point Likert scale ranging from 1 ("strongly disagree") to 4 ("strongly agree"). Other variables such as demographics and year of match or anticipated match were also collected. Respondents were divided into Surgical and Non-Surgical groups with Surgical specialties including general, orthopedic, otolaryngology, neurological, thoracic, plastic, urological, and vascular surgery as well as obstetrics and gynecology. Statistical analyses were calculated using IBM SPSS ver. 27, and $\mathrm{p}$ values 0.05 were considered significant. Descriptive statistics for categorical data were reported as absolute frequencies/percentages and analyzed by a chisquare test. Continuous variables are reported as medians/ interquartile ranges and are analyzed by a student's t-test. Currently there is no literature that explores an osteopathic medical students' decision to pursue general surgery or a subsurgical specialty. By obtaining this data, we hope that it will be used to better inform both OMS and osteopathic medical schools on what is needed to prepare for residency.

Results: A total of 221 completed surveys were received (14.5\%) response rate with $77 \%$ of respondents being labelled as non-surgical and $23 \%$ being labelled as surgical. Respondents were split almost evenly between male (49\%) and female (51\%) with the distribution of males and 
females being uneven in the surgical group, with $67 \%$ of respondents being female. Distribution of females in the non-surgical group was $47 \%$. The majority of respondents were White (67\%), Asian (55\%), or Black (5\%), and 3.6\% were Hispanic or Latino. Both surgical and nonsurgical groups found their clinical rotations important in their decision to pursue their residency ( $96 \%$ vs. $89 \%$; $p=0.18$ ) with both groups having moderate interest in their specialty before starting medical school (67\% vs. 58\%; $\mathrm{p}=0.26)$. USMLE Step 1/COMLEX Level 1 scores were important to both groups ( $87 \%$ vs. $81 \%$; $=0.51$ ) with USMLE Step 2/COMLEX Level 2 scores being more important to the surgical group ( $94 \%$ vs. $76 \%$; p $<0.05)$. Additionally, surgical group was more likely to take both USMLE and COMLEX compared to the non-surgical group (88\% vs. $67 \%$; $\mathrm{p}<0.05)$. Both groups thought having a mentor in the field they applied to would be helpful (100\% vs. $96 \% ; p=0.20$ ) with $55 \%$ of the surgical and $45 \%$ of the non-surgical $(p=0.21)$ having a mentor and $45 \%$ surgical and $32 \%$ non-surgical $(\mathrm{p}=0.14)$ unable to find a mentor. The ability to do research was more important to the surgical group ( $89 \%$ vs. $67 \%$; $p<0.05)$ with the ability to publish papers more important to the surgical group $(71 \%$ vs. $44 \%$; $\mathrm{p}<0.001)$. Both groups believed that hospital affiliations with their school were important ( $56 \%$ vs. $63 \%$; $\mathrm{p}=0.50$ ).

Conclusion: Our study shows that for surgical versus nonsurgical OMS, there were clear differences in perceived barriers to matching to their preferred specialty. Unsurprisingly, students interested in surgery weighted board examinations and research as more important than nonsurgical specialties. While both groups found USMLE Step 1/COMLEX Level 1 scores to be important, the surgical group perceived USMLE Step 2/COMLEX Level 2 to be important for matching to their preferred residency specialty. Likewise, the surgical group perceived the ability to do research as well as the number of publications they author to be important when compared to the non-surgical group. Consistent with studies previously conducted on OMS in primary care fields as well as allopathic students in surgical fields, mentorship and clinical experience were perceived as important to both groups. This study had several limitations including that the survey was only administered to OMS at one school and its branch campus. Additionally, there were less surgical respondents then non-surgical respondents which is to be expected at an osteopathic medical school. Interestingly, there were more female than male surgical respondents despite the fact that there are more male versus female surgical residents, potentially contributing to a selection bias in our results.
Despite these limitations, this study highlights how osteopathic medical schools can better serve the needs of OMS applying to surgical specialties by allowing OMS to potentially participate in research electives as well as the student body as a whole by establishing mentorship programs to improve OMS ability to match to the residency of their choice.

\section{References}

1. Dogbey GY, Collins K, Russ R, Brannan GD, Mivsek M, Sewell S. Factors Associated With Osteopathic Primary Care Residency Choice Decisions. J Am Osteopath Assoc. 2018;118(4):225-233. doi:10.7556/jaoa.2018.046

2. Trinh LN, O'Rorke E, Mulcahey MK. Factors Influencing Female Medical Students' Decision to Pursue Surgical Specialties: A Systematic Review. J Surg Educ. 2021;78(3):836-849. doi:10.1016/ j.jsurg.2020.08.050

3. Drolet BC, Sangisetty S, Mulvaney PM, Ryder BA, Cioffi WG. A mentorship-based preclinical elective increases exposure, confidence, and interest in surgery. Am J Surg. 2014;207(2):179-186. doi:10.1016/j.amjsurg.2013.07.031

4. Shelton J, Obregon M, Luo J, Feldman-Schultz O, MacDowell M. Factors Influencing a Medical Student's Decision to Pursue Surgery as a Career. World J Surg. 2019;43(12):2986-2993. doi:10. 1007/s00268-019-05167-9

5. Schmidt LE, Cooper CA, Guo WA. Factors influencing US medical students' decision to pursue surgery. J Surg Res. 2016;203(1):6474. doi:10.1016/j.jss.2016.03.054

Financial Disclosures: None reported.

Support: None reported.

Ethical Approval: Our study was reviewed and deemed exempt by the NYIT-COM IRB committee. IRB number is BHS -1629.

Informed Consent: No informed consent was needed for this survey based study.

Poster No.: ${ }^{\star} \mathrm{C} 17$

Abstract No.: 56

Category: Clinical

Research Focus Area: Osteopathic Philosophy

Quantification of Palpatory Forces

Exerted During Abdominal

Examination of Generally Healthy Subjects by Practicing Physicians

${ }^{1}$ Kylee Marie Stevenson OMS-II; ${ }^{2}$ Tina T. Wong, OMS-II; ${ }^{2}$ Cesar Yeelot, OMS-II; ${ }^{2}$ Adrienne M. Kania, DO, FAAO 
${ }^{1}$ Burrell College of Osteopathic Medicine (BCOM); ${ }^{2}$ Department of Clinical Medicine, Burrell College of Osteopathic Medicine (BCOM)

Context: Palpation is a complex skill for beginning medical personnel to learn. Standardization of palpation is difficult as pressures exerted during an abdominal exam are unknown without the use of pressure sensors (Hyde et al, 2012). There is a lack of research in standardizing pressures, however, one current manuscript has a similar protocol. The limitation of that research was that it had only one physician using an alternative pressure sensor on limited subjects (Hsu et al., 2020).

Objective: To know typical pressures that are applied while performing an abdominal exam will create a normative database and could be valuable for the instruction of this skill. Not knowing this can easily result in misdiagnosis from beginning medical personnel until they acquire a normative database for the pressures needed to accurately perform the exam (Frellick et al., 2021).

Methods: Thirty-four physicians were recruited to palpate the abdomen of subjects with varying body mass indices (BMI): normal, overweight, and obese. The physicians were consented and instructed to complete a survey that identifies the following factors: gender, years in practice, physician specialties, location of training, and hand dominance. Eighteen subjects were recruited and prescreened to ensure there was no history of various abdominal disorders or prior surgeries. The pressures exerted during the abdominal exam were quantified with Novel LoadPad sensors attached to the palpating hand while examining the four abdominal quadrants, liver, spleen, and kidney. Pressure readings were plotted against varying gender, length of time in practice, physician specialties, US-trained vs non-US-trained physician examiners, and subject BMI. Statistical analysis was completed using Two-Tailed T-tests and Analysis of Variance (ANOVA). Normal distribution curves were formulated using Excel software. By quantifying pressures used during an abdominal examination, we are creating normative data that can be used when evaluating pressures used during visceral manipulation.

Results: The range of pressures used to palpate lightly was $8.8 \pm 3.4 \mathrm{~N}$, liver $17.0 \pm 8.2 \mathrm{~N}$, spleen $16.5 \pm 7.3 \mathrm{~N}$, and kidney $17.6 \pm 7.7 \mathrm{~N}$. There was no difference between men and women when palpating lightly in the four quadrants or kidneys. However, women used lighter pressure when evaluating the liver and spleen, trending toward significance. When evaluating pressures generated during palpation, the years in practice showed no difference for light four-quadrant, spleen, or kidney evaluation, but a significant difference $(\mathrm{p}=0.028)$ when palpating the liver. Generally, the longer a physician is in practice, the less pressure is used. When evaluating the data based on clinical specialty, those specialized in Osteopathic Neuromuscular Manipulative Medicine (ONMM) tended to use lighter pressures. There was a statistically significant difference in pressure used for palpation based on whether a physician did their training in the US or abroad when assessing the spleen $(p=0.03)$. Non-US-trained physicians used lighter pressures than US-trained physicians. There was no distinction for light palpation in four quadrants or spleen in subjects regardless of their BMI, but did show a trend for less force applied for the liver and kidney evaluation for those subjects with normal BMI.

Conclusion: There is a wide range of pressures used when performing an abdominal exam. The results acquired during this study confounds recommendations for teaching methods for those in the healthcare profession. This suggests a need for more specificity of pressures when performing an abdominal examination. Women, ONMM specialists, and non-US based-trained physicians tended to use lighter pressures, regardless of subject BMI. The limitations of this study include the narrow variation of BMI of the subjects, the limited number of subjects evaluated per physician participant, few participants in various specialties, such as those who are in pediatrics, surgery, and emergency medicine, and uncertainty as to whether the organ in question was truly palpated. Future studies could be conducted utilizing ultrasound technology to determine when the organ is being contacted by the physician. A larger-based study that includes more variety in physician specialty and more variability in subjects could alleviate these limitations.

\section{References}

1. Hyde L, Erolin C, Ker J. Creation of abdominal palpation model prototype for training of medical students in detection and diagnosis of liver disease. Journal of Visual Communication in Medicine. 2012;35(3):104-114. doi:10.3109/17453054.2012.713855

2. Hsu JL, Lee $\mathrm{CH}$, Hsieh $\mathrm{CH}$. Digitizing abdominal palpation with a pressure measurement and positioning device. PeerJ. 2020;8:e10511. Published 2020 Dec 17. doi:10.7717/peerj.10511

3. Frellick, M., \& Vega, C. P. How Big a Problem Is Misdiagnosis in Medicine? Medscape. Accessed July 9, 2021. http://www. medscape.org/viewarticle/933116

Financial Disclosures: Dr. Kania is a co-investigator on the AOA grant (\#19137759), "Anti-Inflammatory Actions of OMT - Role of the Cholinergic Anti-Inflammatory Reflex 
and Translocation of Immune Cells from Reticular Organs to the Systemic Circulation," and is the mentor for $\mathrm{t}$

Support: This study was funded by Burrell College of Osteopathic Medicine Summer Research Experience, 2021. Compensation for subjects was $\$ 15$ per hour of time committed to the study.

Ethical Approval: This study was deemed reviewed and approved. IRB Approval: Burrell IRB 0080-2021.

Informed Consent: Participants: Physicians gave their consent to have the pressure they generated when performing abdominal exams recorded and completed a questionnaire located on a secured server that documented handedness, years in practice, gender, specialty, and licensure. The consent form and questionnaire were approved by the IRB committee. Test Subjects: Subjects were screened for previous abdominal surgery or abdominal disorders. This information was obtained via a questionnaire on a secured server. If eligible, the subjects were informed of the study protocol and gave their consent. Both the consent and questionnaire were approved by the IRB committee.

Poster No.: ${ }^{\star} \mathrm{C} 18$

Abstract No.: 59

Category: Clinical

Research Focus Area: Impact of OMM \& OMT

AOA Grant Award: 431607710

\section{Serum osteocalcin in the} osteopathic treatment for motor function in Parkinson's Disease

${ }^{1}$ Swati Gupta, OMS-II; ${ }^{2}$ Sheena Lamba, OMS-II; ${ }^{2}$ Sheldon

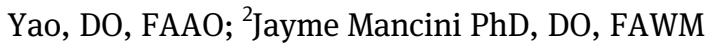

${ }^{1}$ New York Institute of Technology (NYITCOM); ${ }^{2}$ Osteopathic Manipulative Medicine, New York Institute of Technology (NYITCOM)

Context: Osteocalcin (OCN) is secreted by osteoblasts to facilitate bone development and remodeling in response to mechanical stress. It also stimulates anabolic processes such as improvement in age-related nervous system decline in humans. ${ }^{1}$ Treatment with $\mathrm{OCN}$ was protective against neurodegeneration in a Parkinson's disease (PD) rat model. ${ }^{2}$ Although OCN may be a useful biomarker of bone stimulation by osteopathic manipulative medicine (OMM) in PD, serum OCN has not been studied in PD.

Objective: To determine if OCN levels in individuals with $\mathrm{PD}$ are associated with disease-severity.

Methods: This observational cross sectional study of correlations of OCN to validated assessment of severity of motor function in PD (MDS-UPDRS-part 3), sensory organization testing (SOT), and activities of daily living in PD (PDQ39) is part of an IRB-approved (BHS 975) randomized controlled trial of OMM for motor function and balance in PD. ${ }^{3}$ Of the 33 individuals participating in the trial, 10 subjects with a Hoehn \& Yahr of 2 were selected. Their pre-intervention OCN serum levels were analyzed by Life Extension-Labcorp. Serum OCN was statistically tested for Spearman's correlation (2-tailed p-value) with demographics and functional measures.

Results: The mean age was $67.4( \pm 5.5)$ years. Participants had PD for 1-17 years and were $80 \%$ male; $20 \%$ female. Mean serum OCN was $16( \pm 7.8) \mathrm{ng} / \mathrm{ml}$. Serum OCN correlations were age $r s=-0.1515(\mathrm{p}=.676)$, BMI $\mathrm{rs}=-0.1849$ $(\mathrm{p}=.634), \quad$ MDS-UPDRS-part3 $\mathrm{rs}=0.7699 \quad(\mathrm{p}=.015)$, SOT $\mathrm{rs}=0.1982 \quad(\mathrm{p}=.670), P D Q 39 \mathrm{rs}=0.2833 \quad(\mathrm{p}=.460)$ and the number of years having PD rs=0.2594 ( $\mathrm{p}=.500)$.

Conclusion: Serum OCN levels were within the established lab reference range for healthy individuals. Serum OCN significantly, positively correlated with severity of motor function impairment. This subset of participants came from a study in which there was significant improvement on MDS-UPDRS-part 3 in the OMM group. The lack of correlation to activities of daily living in PD and balance suggests that these would not be confounding variables in serum OCN if used as a biomarker for mechanical stress into bone during OMM. Further analysis is required to determine if release of $\mathrm{OCN}$ was part of the therapeutic effect.

\section{References}

1. Shan C, Ghosh A, Guo X, et al. "Roles for osteocalcin in brain signalling: implications in cognition- and motor-related disorders." Molecular brain, 2019; 12(1): 23. https://doi.org/10.1186/ s13041-019-0444-5

2. Guo X, Shan C, Hou Y, Zhu G, Tao B, Sun L, Zhao H, Ning G, Li S and Liu J. Osteocalcin Ameliorates Motor Dysfunction in a 6-Hydroxydopamine-Induced Parkinson's Disease Rat Model Through AKT/GSK3 $\beta$ Signaling. Front. Mol. Neurosci. 2018; 11:343. https://doi.org/10.3389/fnmol.2018.00343

3. DiFrancisco-Donoghue J, Apoznanski T, de Vries K, Jung MK, Mancini J, Yao S. Osteopathic manipulation as a complementary approach to Parkinson's disease: A controlled pilot study. NeuroRehabilitation. 2017;40(1):145-151. doi:10.3233/NRE-161400

Financial Disclosures: The authors have no financial interest or conflict of interest in relation to this abstract to disclose. 
Support: American Academy of Osteopathy LBORC External Grant to Jayme Mancini 2020; American Osteopathic Association (AOA) 2016 Research Grant

Ethical Approval: Approved by NYIT IRB BHS975

Informed Consent: Research participants consented by IRB-approved consent form.

Poster No.: *C19

Abstract No.: 66

Category: Clinical

Research Focus Area: Osteopathic Philosophy

\section{Efficacy of High Titer vs. Low Titer Convalescent Plasma against SARS-CoV-2 in a Rural Hospital}

${ }^{1}$ James Katsilometes, OMS-II; ${ }^{2}$ Rondon Clavo Carlos, MD PGY 2; ${ }^{3}$ Anthony Santarelli, PhD; ${ }^{3}$ John Ashurst, DO; ${ }^{3}$ Dietrich Tyson, PharmD

${ }^{1}$ Pacific Northwest University of Health Sciences College of Osteopathic Medicine (PNWU-COM); ${ }^{2}$ Emergency Medicine, Kingman Regional Medical Center; ${ }^{3}$ General Medical Education, Kingman Regional Medical Center

Context: As of June 24, 2021, 177,866,160 cases of SARS-CoV-2 infection had been reported globally with an overall mortality count of $3,857,974 .{ }^{1}$ A combination of therapies are administered to hospitalized patients to mitigate disease progression. One treatment, convalescent plasma (CP), may reduce mortality and appears safe. ${ }^{3}$ Limitations in the availability of convalescent plasma has required community hospitals to seek multiple suppliers of CP. Thus, neutralizing antibody levels are of varying titer.

Objective: To determine if $\mathrm{CP}$ containing high titer of neutralizing antibodies was associated with better clinical improvement in hospitalized patients with SARS-CoV-2 infection when compared to those received low titer.

Methods: A retrospective chart review of all patients administered CP for nucleic acid positivity of SARs-CoV-2 via a nasopharyngeal sample from November 2020 to June 2021 was conducted. Neutralizing Antibody (NAb) titer of was determined via anti-SARS-CoV-2 IgG chemiluminescent immunoassay. ${ }^{2} \mathrm{CP}$ was then categorized as High titer (HT; IgGNAb>1;1000) or Low titer (LT; IgGNAb <1:1000). Patients were then matched on demographics and comorbidities to patients receiving LT CP. Primary outcome was defined 28-day mortality. Secondary outcomes were; number of days hospitalized, 28-day readmission, and 28-day admission to the ICU. The results are were assessed for significance with the chi-squared and Mann-Whitney test.

Results: A total of 62 participants, 31 in each cohort, were included in the final analysis and 54.5\% (34/62) were female. A minority of patients were transferred to the ICU $21.0 \%(13 / 62)$ or expired $19.4 \%$ (12/62). The median age of patients enrolled in the study was 64.5 years old; (58-75.25). The most common comorbidity was hypertension 30/62 (48.4\%), followed by obesity $38.7 \%$ (24/62). No significant difference between HT and LT cohorts was detected for age $(\mathrm{p}=0.667)$, body mass index $(\mathrm{p}=1.000)$, or multiple comorbidities $(\mathrm{p}=0.799)$. Patients administered HT CP had a median length of stay in the hospital of 5 days (4-8) while patients receiving LT CP had a median length of stay of 8 days $(5-18)(\mathrm{p}=0.034)$.

Conclusion: Of the participants who received convalescent plasma upon hospitalization with a diagnosis of SARS-CoV-2, high titer of NAb should be preferred over convalescent plasma with low titer. The reduction in hospital length of stay may extend to other rural community hospitals.

\section{References}

1. WHO. Coronavirus Disease (COVID-2019) Situation Report, 22.06.2021. Geneva. 2021. https://www.who.int/emergencies/ diseases/novel-coronavirus-2019/situation-reports/. Accessed 24.06.2021.

2. Theel ES, Harring J, Hilgart H, Granger D. Performance Characteristics of Four High-Throughput Immunoassays for Detection of IgG Antibodies against SARS-CoV-2. J Clin Microbiol. 2020;58(8):e01243-20. Published 2020 Jul 23. doi:10.1128/ JCM.01243-20

3. Mair-Jenkins J, Saavedra-Campos M, Baillie JK, et al. The effectiveness of convalescent plasma and hyperimmune immunoglobulin for the treatment of severe acute respiratory infections of viral etiology: a systematic review and exploratory metaanalysis. J Infect Dis. 2015;211(1):80-90. doi:10.1093/infdis/ jiu396

4. Joyner MJ, Carter RE, Senefeld JW, et al. Convalescent Plasma Antibody Levels and the Risk of Death from Covid-19. N Engl J Med. 2021;384(11):1015-1027. doi:10.1056/NEJMoa2031893

5. https://www.accessdata.fda.gov/scripts/cdrh/cfdocs/cfcfr/ CFRSearch.cfm? $\mathrm{fr}=640.65$ (Accessed on June 25, 2021).

6. “AZDHS: Epidemiology \& Disease Control - Covid 19 - Data." Arizona Department of Health Services, www.azdhs.gov/ covid19/data/index.php\#hospital-bed-usage.

7. Pijls, B. G., Jolani, S., Atherley, A., Derckx, R. T., Dijkstra, J., Franssen, G., Hendriks, S., Richters, A., Venemans-Jellema, A., Zalpuri, S., \& Zeegers, M. P. (2021). Demographic risk factors for COVID-19 infection, severity, ICU admission and death: a metaanalysis of 59 studies. BMJ open, 11(1), e044640. https://doi. org/10.1136/bmjopen-2020-044640 
8. 2. Gallo Marin, B., Aghagoli, G., Lavine, K., Yang, L., Siff, E. J., Chiang, S. S., Salazar-Mather, T. P., Dumenco, L., Savaria, M. C., Aung, S. N., Flanigan, T., \& Michelow, I. C. (2021). Predictors of COVID-19 severity: A literature review. Reviews in medical virology, 31(1), 1-10. https://doi.org/10.1002/rmv.2146

9. 3. Du Y, Lv Y, Zha W, Zhou N, Hong X. Association of body mass index (BMI) with critical COVID-19 and in-hospital mortality: A dose-response meta-analysis. Metabolism. 2021 Apr;117:154373. doi: 10.1016/j.metabol.2020.154373. Epub 2020 Sep 16. PMID: 32949592; PMCID: PMC7493748.

10. 4. Rajpal A, Rahimi L, Ismail-Beigi F. Factors leading to high morbidity and mortality of COVID-19 in patients with type 2 diabetes. J Diabetes. 2020 Dec;12(12):895-908. doi: 10.1111/17530407.13085. Epub 2020 Sep 2. PMID: 32671936; PMCID: PMC7405270.

11. Shah P, Owens J, Franklin J, et al. Demographics, comorbidities and outcomes in hospitalized Covid-19 patients in rural southwest Georgia. Ann Med. 2020;52(7):354-360. doi:10.1080/ 07853890.2020 .1791356

12. Ricketts TC. The changing nature of rural health care. Annu Rev Public Health. 2000;21:639-57. doi: 10.1146/annurev.publhealth. 21.1.639. PMID: 10884968.

13. "Therapeutics and COVID-19: Living Guideline." World Health Organization, World Health Organization, www.who.int/ publications/i/item/WHO-2019-nCoV-therapeutics-2021.1.

Financial Disclosures: None reported.

Support: None Reported.

Ethical Approval: IRB \#0189

Informed Consent: I hereby consent, James Katsilometes

Poster No.: * $\mathrm{C} 20$

Abstract No.: 71

Category: Clinical

Research Focus Area: Osteopathic Philosophy

Improving Upon Osteopathic Palpatory Skills using a 3D Printed Rotational Lumbar Spine Model

${ }^{1}$ Tiffany Rey, OMS IV; ${ }^{2}$ Adam Thomas, OMS-III; ${ }^{3}$ James Nolin, FNP; ${ }^{2}$ J. Gage Sanders, OMS III; ${ }^{2}$ Kaitlyn Cedoz, OMS-III; ${ }^{2}$ Rheketah Berwick, OMS IV

${ }^{1}$ Alabama College of Osteopathic Medicine (ACOM); ${ }^{2} \mathrm{ACOM}$ Fellowship, Alabama College of Osteopathic Medicine (ACOM); ${ }^{3}$ Department of Clinical Sciences, Alabama College of Osteopathic Medicine (ACOM)

Context: Most osteopathic medical schools teach students intersegmental motion testing as a part of diagnosing somatic dysfunctions of the spine. Previous studies have investigated the palpatory skills of medical students in a transverse plane using a static lumbar spine model. ${ }^{1}$ One way in which students can learn and practice palpatory skills is through the use of 3D printed models. ${ }^{2}$ Many pathologies and examples can be printed, reducing cost and time needed to train students.

Objective: To design and validate an improved instructional model to teach intersegmental diagnosing using a 3D printed rotational lumbar spine.

Methods: Using a 3D printer, a lumber spine was constructed. A layer of dense foam and artificial silicone skin was placed overlying the 3D printed spine to simulate the texture of muscles and skin. These segments were fixated using a bronze stud that travelled through the body of each segment to allow for rotation in a transverse axis of each segment. For the purpose of the study, washers were used between the segments to mimic the joint space of each segment. Additionally, facet joints were cut to allow independent rotation of each segment rather than introducing group mechanics of the entire model.

Springs and threaded rods were mounted onto the transverse processes of each segment. Nuts were placed on the front and back of the threads as they were mounted into the framework. Segments were placed at $0 \mathrm{~mm}, 2 \mathrm{~mm}$, $4 \mathrm{~mm}, 6 \mathrm{~mm}$, and $8 \mathrm{~mm}$ of asymmetry based on the measured amount of thread behind the mounted structure, with $0 \mathrm{~mm}$ serving as a control. Segments and rotation (left or right) were assigned randomly for two different sessions.

First, second-, and third-year medical students were invited to participate in the study with no exclusion criteria. Students were instructed to determine the orientation of the lumbar segments based on rotation and filled out a survey to calculate results. Two trials were completed with randomization of segment rotation for each trial. 82 students participated in the first trial, and 33 students participated in the second trial. An ANOVA was used to determine which segment was considered significant compared to the control. A two-way ANOVA was used to determine significance in relation to OMS status or gender. This study holds osteopathic significance as its goal is to validate a lumbar spine model for students to use in the future to practice palpatory skills.

Results: A total of 115 trials (87 OMS I, 25 OMS II, 3 OMS III) were performed with no exclusions. GraphPad Prism version 9.1 was used for statistical analysis. The ratio of correct to incorrect identification of rotation at $0 \mathrm{~mm}, 2 \mathrm{~mm}, 4 \mathrm{~mm}$, $6 \mathrm{~mm}$, and $8 \mathrm{~mm}$ were $0.51+/-0.046,0.27+/-0.042,0.25+/-$ $0.040,0.34+/-0.045$, and $0.46+/-0.047$, respectively. A One-way AVOVA with a Dunnett post-hoc test was used to compare statistical difference from the $0 \mathrm{~mm}$ control revealing $2 \mathrm{~mm}$ ( $\mathrm{p}=0.0008), 4 \mathrm{~mm}(\mathrm{p}=0.0001)$, and $6 \mathrm{~mm}$ $(p=0.0298)$ were statistically significant. The $8 \mathrm{~mm}$ segment was not statistically significant $(\mathrm{p}=0.82)$ compared to the 
control. A two-way ANOVA was not significant when comparing OMS I and II years $(\mathrm{p}=0.29)$.

Conclusion: The goal of this study was to determine if a more accurate model could be developed to better teach students the principles of osteopathic manipulative medicine as related to intersegmental motion testing of the lumbar spine. When polling OMS I, II and III students with two different trials, a total of 115 students performed the experiment with no final exclusion criteria. When compared to the control, students were just as likely to determine rotation of a spinal segment at $8 \mathrm{~mm}$ of rotation. Students did not perform as well when segments were rotated at $2 \mathrm{~mm}, 4 \mathrm{~mm}$ and $6 \mathrm{~mm}$ as determined by a oneway ANOVA.

Many limitations were witnessed throughout the trials of this study. Correct location of landmarks was the largest challenge experienced by students as there was no guidance given for the location of the segments within the apparatus. Additionally, the level of interest was varied among different cohorts of students based on the time of the year that the study was conducted.

Future research is expected to be performed to investigate some of these limitations to include; telling students where to palpate the transverse processes, removal of the muscle and skin layer to introduce more of a layered palpation approach to the model, and to be performed blindfolded.

This study was used to determine the amount of rotation needed for future models to be calibrated and appropriately allow students to learn how to palpate. Additionally, a validated model could be explored in clinical encounters or simulation settings for students to use.

\section{References}

1. Snider EJ, Pamperin K, Pazdernik V, Degenhardt BF. Influence of transverse process landmark localization on palpation accuracy of lumbar spine models. Journal of Osteopathic Medicine. 2018;118(3):151-158. doi:10.7556/jaoa.2018.034

2. Han $M$, Portnova AA, Lester $M$, Johnson $M$. A do-it-yourself 3D-printed thoracic spine model for anesthesia resident simulation. Meyer MJ, ed. PLoS ONE. 2020;15(3):e0228665. doi:10. 1371/journal.pone.0228665

Financial Disclosures: None reported.

Support: None reported.

Ethical Approval: Reviewed and approved. IRB\# HS210128-EX
Informed Consent: Consent was obtained from each participant with an option to withdraw at any given time.

Poster No.: ${ }^{\star} \mathrm{C} 21$

Abstract No.: 75

Category: Clinical

Research Focus Area: Chronic Diseases \& Conditions

Inflammatory Bowel Disease: Genitourinary Complications in a Cross-Race Analysis

${ }^{1}$ Jake Herbert, OMS-IV; ${ }^{1}$ Landen Burstiner, OMS-IV; ${ }^{1}$ Ralfi Doka, OMS-IV; ${ }^{2}$ Emily Teeter, BS; ${ }^{3}$ Amor Royer, MD; ${ }^{3}$ Anna H. Owings, DO; ${ }^{4}$ Julia Liu, MD; ${ }^{5}$ Sarah C. Glover, DO; ${ }^{5}$ Pegah Hosseini-Carroll, MD

${ }^{1}$ Nova Southeastern University Dr. Kiran C. Patel College of Osteopathic Medicine (NSU-KPCOM); ${ }^{2}$ College of Liberal Arts and Sciences, University of Florida; ${ }^{3}$ Department of Internal Medicine, University of Mississippi Medical Center; ${ }^{4}$ Division of Gastroenterology, Morehouse School of Medicine; ${ }^{5}$ Department of Digestive Disease, University of Mississippi Medical Center

Context: Inflammatory Bowel Diseases (IBD), like ulcerative colitis (UC), are associated with numerous extra-intestinal manifestations (EIM). Commonly studied genitourinary (GU) EIM's found in Crohn's disease (CD) include urolithiasis, urinary tract infections (UTI's), and cystitis. ${ }^{1}$ Literature reviewed for this study identifies an increased association of CD and urolithiasis against the general population. ${ }^{2}$ The rate of GU comorbidities has not been well characterized in cross-race analyses.

Objective: To establish the proliferation of common GU comorbidities in $\mathrm{CD}$ and UC and to further determine at what rate these affect the African American (AA) and Caucasian (CA) populations.

Methods: This is a retrospective cohort study using data collected from a research data base that included 6 integrated, tertiary healthcare facilities from 2012 to 2020. The electronic chart records for 3104 CA and AA IBD patients were reviewed for incidences of urolithiasis, UTI, and cystitis via diagnosed ICD-10 codes. Comparison between data groups was made using t-tests and chi square tests.

Results: Our study included 3,104 patients of which 38\% were AA, 59\% female, and 43\% diagnosed with UC. Similar 
proportions of UC and CD diagnosed patients developed urolithiasis $(6.0 \%$ vs $6.7 \%, \mathrm{p}=0.46)$, as well as cystitis and UTI's ( $5.6 \%$ vs. $4.3 \%, \mathrm{p}=0.11 ; 14.0 \%$ vs. $12.9 \%, \mathrm{p}=0.35$, respectively). Additionally, fistula formation in $\mathrm{CD}$ was associated with a 2.80 times greater chance of UTI's (95\% CI 2.077-3.785, $\mathrm{p}<0.001)$. Similar proportions of AA and CA patients developed urolithiasis ( $5.5 \%$ vs $7.0 \%, p=0.09$ ) with AA's showing a higher risk of developing UTI's and cystitis $(17.0 \%$ vs $11.1 \%, p=<0.001 ; 6.2 \%$ vs $4.0 \%, p=0.006$, respectively).

Conclusion: This study found that there were similar rates of urolithiasis formation in both $\mathrm{UC}$ and $\mathrm{CD}$. Furthermore, these rates were not significantly different between AA and CA IBD populations. This could potentially represent that patients with UC have a similarly elevated risk of urolithiasis as those with CD. Further studies of patients with UC against a demographicmatched control cohort would help to clarify these findings. Additionally, there were similar rates of UTI's and cystitis formation in both UC and CD. However, AA's with IBD have a significantly higher burden of UTI and cystitis complications as compared to their CA cohorts. This could possibly be due to health disparities between the $\mathrm{AA}$ and CA populations. These findings suggest physicians should be vigilant about screening all IBD patients with renal dysfunction for urolithiasis and UTI's to prevent severe complications like renal failure and sepsis. ${ }^{3}$

\section{References}

1. Gaspar SR, Mendonca, T, Oliveira P, et al. "Urolithiasis and Crohn's Disease." Urol Ann, vol. 8, no. 3, 2016, pp. 297-304, doi:10.4103/0974-7796.184879.

2. Larsen S, Bendtzen K, Nielsen OH. "Extraintestinal Manifestations of Inflammatory Bowel Disease: Epidemiology, Diagnosis, and Management.” Annals of Medicine, vol. 42, no. 2, 2010, pp. 97114, doi:10.3109/07853890903559724.

3. Varda BK., McNabb-Baltar J, Sood A, et al. "Urolithiasis and urinary tract infection among patients with inflammatory bowel disease: a review of US emergency department visits between 2006 and 2009." Urology, Volume 85, Issue 4, 2015, Pages 764-770, doi:10. 1016/j.urology.2014.12.011.

Financial Disclosures: None reported.

Support: None reported.

Ethical Approval: IRB File \#2019-0194

Informed Consent: N/A

Poster No.: ${ }^{\star} \mathrm{C} 22$

Abstract No.: 77
Category: Clinical

Research Focus Area: Chronic Diseases \& Conditions

\section{A Modified Charlson Comorbidity Index Scoring System Aids in Trauma Mortality Prediction and Population Health Improvement}

${ }^{1}$ Sonia Amanat OMS III; ${ }^{2}$ Stephanie De Mel, OMS-III; ${ }^{2}$ Dana Schulz, OMS-III; ${ }^{2}$ Scott Kivitz, OMS-III; ${ }^{2}$ Taner B. Celebi, OMS-III; ${ }^{2}$ Stephen DiRusso, PhD, MD

${ }^{1}$ New York Institute of Technology (NYITCOM); ${ }^{2}$ Department of Trauma Surgery, New York Institute of Technology (NYITCOM)

Context: This project will illustrate a modified Charlson Comorbidity Index (CCI) and it's use in the mortality correlation of trauma patients in a level 2 trauma center. The original CCI was updated to fit the demographic profile of the patients admitted to the trauma center. The update includes an increased comorbidity profile and numerical weighted system. This modified CCI may aid in identifying areas of improvement for comorbid conditions and trauma outcomes.

Objective: The primary objective of this study is to develop a modified CCI that takes into account multiple comorbidities that can affect trauma patients.

The secondary objective is to utilize the modified CCI to predict mortality.

Methods: This is a retrospective cross sectional study of 4,391 trauma patients at level 2 trauma center St. Barnabas Hospital in Bronx, New York. Patients who were dead on arrival were removed from the data set. The creation of the modified CCI began with the original CCI's framework. Additional comorbidities found within the trauma database were incorporated based on similarities to previously included comorbidities. The weighting system was retained in order to place emphasis on potentially more severe comorbidities. A total of 15 comorbidities were added to the original CCI creating the modified one.

Using SPSS (IBM Version 26), a binomial logistic regression was run to determine the association between the modified CCI and outcome of death. The Hosmer-Lemeshow test was used to assess goodness of fit of the single-predictor model, while the odds ratio was a determinant of the association. Column proportion tests were used to determine if a 
relationship exists between the modified CCI and outcome of death, and if there are intergroup differences. Modified CCI groups were developed based on the original CCI (mild $=1-2$; moderate $=3-4$; and severe $=\geq 5$ ).

Results: The modified CCI is associated with an outcome of death (Chi-squared=38.23, $\mathrm{p}<.001$ ). The model based solely on modified CCI is a good fit for the data $(\mathrm{HL}=14.32, \mathrm{p}<.05)$. Modified CCI could be used as an independent predictor of death for trauma cases at SBH based on available data, however the model is only a moderate classifier with an auROC of .635 (95\% CI: 0.583, 0.687). Column proportions were significant for Moderate and Severe vs Mild, but there was not a significant difference between moderate and severe.

Conclusion: The modified CCI has promise when incorporated into trauma mortality prediction. Modification of the weighting system and the expansion of the original CCI illustrates a positive correlation with an outcome of death in the trauma patients. Additionally, the modified CCI allows for appropriate categorization of comorbidities that influence patient prognosis. Changing the severity scale to combine moderate and severe could result in a better predictor. Evaluation of the modified CCI can be assessed as a predictive measure for mortality by incorporation into existing trauma metrics (i.e., TRISS) or in a new predictive model. A comparison study between the original CCI and modified CCI is necessary to conclude the significance of changing the metric. This initial study demonstrates the possible utility of the CCI in improving trauma patient outcomes in the hospital service area, highlighting the abundance of comorbidities affecting the local population.

\section{References}

1. Champion HR. Trauma Scoring. Scandinavian Journal of Surgery. 2002;91(1):12-22. doi:10.1177/145749690209100104

2. Lefering R. Trauma scoring systems. Current Opinion in Critical Care. 2012;18(6):637-640. doi:10.1097/mcc.0b013e3283585356

3. Sundararajan V. New ICD-10 version of the Charlson comorbidity index predicted in-hospital mortality. Journal of Clinical Epidemiology. 2004;57(12):1288-1294. doi:10.1016/j.jclinepi.2004. 03.012

Financial Disclosures: None reported.

Support: None reported.

Ethical Approval: N/A

Informed Consent: N/A
Poster No.: ${ }^{\star} \mathrm{C} 23$

Abstract No.: 78

Category: Clinical

Research Focus Area: Chronic Diseases \& Conditions

\section{A Retrospective Analysis of Coronavirus in the Pediatric Population}

${ }^{1}$ Adalee Gardner OMS-IV; ${ }^{2}$ Samantha Hlebak, OMS-IV; ${ }^{2}$ Hannah Simpson; ${ }^{3}$ Hanna Sahhar, MD

${ }^{1}$ Edward Via College of Osteopathic Medicine (South Carolina); ${ }^{2}$ Department of Pediatrics, Edward Via College of Osteopathic Medicine (South Carolina); ${ }^{3}$ Department of Pediatrics, Spartanburg Medical Center

Context: Coronaviruses are among the common acute respiratory infections that children acquire. The differences in how the subtypes of Coronavirus present clinically and the complications patients experience when infected with them have not been characterized thoroughly. This study delineates the clinical presentation, disease severity, and demands of care associated with each of the five Coronavirus subtypes commonly seen in the pediatric population.

Objective: 1 . Describe and analyze how Coronavirus subtypes (OC43, 229E, NL63, HKU1, and SARS-CoV-2) vary in clinical presentation and disease outcome in pediatric patients.

2. Analyze respiratory support required, length of stay, and potential complications in regards to each Coronavirus subtype.

3. Analyze each viral subtype to determine any predisposition to having a more severe course of infection, requiring admission to Pediatric Intensive Care Unit (PICU).

Methods: This retrospective, observational study conducted in a single hospital center reviewed 85 pediatric patients who were admitted over the period from 10/01/ $2016-07 / 07 / 2021$ to the pediatric ward or PICU at Spartanburg Medical Center with a Coronavirus subtype. The subtypes reviewed include: OC43, 229E, NL63, HKU1, and SARS-CoV-2. The criteria for inclusion in the study was a diagnosis of the before mentioned Coronavirus Subtypes in patients under the age of 18 years. The presence of the virus was confirmed with polymerase chain reaction based 
testing, specifically the FilmArray Respiratory panel which identifies common viral and bacterial pathogens that cause upper respiratory infections. The patients in this study were identified through data management software and stratified by Coronavirus subtype in order to perform data analysis. By doing so, the following data was collected: Coronavirus subtype (OC43, 229E, NL63, HKU1, and SARS-CoV2), demographics (age, gender, race, zip code), month of patient diagnosis, exposure risk, significant past medical history (prematurity, immunodeficiency, congenital disease, etc), type of hospital admission (pediatric ward or PICU), length of hospitalization stay, initial clinical presentation, concomitant infection in addition to coronavirus (other viruses detected), clinical management, complications during time of stay, and patient outcome (discharge, transfer to higher level of care, death). The data was then analyzed to determine patterns among the five Coronavirus subtypes.

Results: The data collected shows that $31.6 \%$ of patients across all Coronavirus subtypes presented with nonspecific upper respiratory symptoms. Of the patients in Coronavirus subtype NL63, 17\% presented with croup symptoms. The most widely recognized concomitant infections among all subtypes were Respiratory Syncytial Virus (RSV) and rhino-/enterovirus. The most frequently diagnosed subtype over the study period was OC43. Subtype OC43 resulted in $30 \%$ PICU admissions, resulting in greater patient management demands.

Conclusion: This retrospective, observational study conducted within a single hospital center contributes to understanding the presenting symptomology and severity of the Coronavirus subtypes within the pediatric population. Conducting additional studies using an increased sample size within multiple hospital facilities would be beneficial in supporting the data obtained and contribute to further understanding of the clinical course of these subtypes.

\section{References}

1. Kuypers J, Martin ET, Heugel J, Wright N, Morrow R, Englund JA. Clinical Disease in Children Associated With Newly Described Coronavirus Subtypes. PEDIATRICS. 2007;119(1). doi:10.1542/ peds.2006-1406

Financial Disclosures: None reported.

Support: None reported.

Ethical Approval: IRB reviewed and approved. IRB \# 1705136-1

Informed Consent: N/A
Poster No.: ${ }^{\star} \mathrm{C} 24$

Abstract No.: 81

Category: Clinical

Research Focus Area: Chronic Diseases \& Conditions

\section{The Relationship Between Glycosylated Hemoglobin and Adverse Childhood Experiences in Resource Poor Communities in Lima, Peru.}

${ }^{1}$ Kaitlyn E. Eckert, OMS-IV; ${ }^{2} J a m i e$ Eckert, MPH, MS-I; ${ }^{3}$ Laura Jensen, MPH; ${ }^{4}$ Joseph Bianco, PhD; ${ }^{5}$ David Drozek, DO, FACLM

${ }^{1}$ Ohio University Heritage College of Osteopathic Medicine (OU-HCOM); ${ }^{2}$ Department of Medical Education, Ohio State University College of Medicine; ${ }^{3}$ Department of Lifestyle Medicine, Springfield Health Center; ${ }^{4}$ Department of Social Medicine, Ohio University Heritage College of Osteopathic Medicine (OU-HCOM); ${ }^{5}$ Department of Specialty Medicine; Department of Surgery, Ohio University Heritage College of Osteopathic Medicine (OU-HCOM)

Context: Repeatedly, the adverse childhood experience (ACE) scale significant predicts poor health outcomes. High ACE scores are associated with obesity, Type 2 Diabetes Mellitus (T2DM) and decreased longevity. ${ }^{1-4}$ In 2009, the World Health Organization called for more ACE research in developing countries. Since, research has targeted impoverished and rural communities; yet 10 years later no South American data exists. We seek to evaluate ACE association with HbA1c in select Lima, Peru neighborhoods. Objective: Research Question: Is there a correlation between glycosylated hemoglobin (HbA1c) and adverse childhood experience (ACE) score in resource poor communities of semi-urban Lima, Peru?

Objective: To examine the relationship between selfreported ACEs and finger-stick $\mathrm{HbA1c}$ in a population not previously assessed

Aim 1: To establish ACE prevalence and type disclosed in current sample

Aim 2: To assess relationship of ACE score and T2DM based on HbA1c

Aim 3: To inform patient-centered, holistic care Methods: Between July 8-18, 2018 data was collected from individuals who presented for care at the Ohio University Heritage College of Osteopathic Medicine (OUHCOM) 
medical brigade in five resource poor communities in Lima, Peru. Clinic location and dates were discussed in community worship centers and spread by word of mouth. Our study stemmed from a longitudinal program which visits these communities yearly allowing for growth in community trust and participation. Subjects were consented adults ( $\mathrm{n}=318$; 18-89 yrs) with baseline variables of age, gender, BMI, and past medical history collected. A modified ACE module derived from the Behavioral Risk Factor Surveillance Survey was self-administered in Spanish. HbA1c was determined by finger-stick with standard Center for Disease Control (CDC) cut-off points used for diabetic and BMI (body mass index) categories. BMI was calculated as $\mathrm{kg} / \mathrm{m} 2$. Incomplete ACE surveys were excluded from data analysis. Descriptive and inferential statistical analysis was conducted using SPSS with univariate t-tests and Pearson Correlation Coefficient administered. The relationship between HbA1c and ACE score was evaluated in efforts to better understand and promote patient-centered care based on the four tenets of osteopathy.

Results: Our investigation failed to show a relationship between ACEs and health outcomes previously found in other resource poor communities. When controlling for BMI, sex, and age, there was no statistical significance between ACE scores and risk of T2DM $(r=.09)$. Nor was there statistical significance between ACE and BMI $(\mathrm{r}=0.1)$ or BMI and HbA1c $(\mathrm{r}=0.21)$. Greatly limiting analysis, sample size was reduced from $n=471$ to $n=318$ after removing incomplete ACE surveys. Of the ten questions asked on the modified survey, domestic violence in the home (42.8\%) and physical or emotional abuse (50.3\%) had the most confirmatory answers, designated as any answer excluding "never." Females ( $M=45.6$ yrs) reported greater ACE exposure than males ( $M=51.6 \mathrm{yrs})$, but were heavily represented in this sample (82.4\% Female). There is a trend of increasing ACE scores and HbA1c approaching a positive association from scores 0-5, however scores 6-9 digress from this trend. Overall, there was low ACE scores (range: $0-8$, avg=2.53) across all diabetic classifications: normal HbA1c (avg ACE=2.55); Prediabetic ( $n=49$, avg ACE=2.76); T2DM ( $n=33$, avg ACE $=2.09$ ). With $71.7 \%$ of participants reporting ACE scores 0-3 and only 6.3\% reporting scores above 6 , these results opposed current theories concerning childhood trauma and adult health outcomes.

Conclusion: This study aimed to measure the relationship between adverse childhood experiences and HbA1c in a population not previously assessed. Previous research showed a dose-response correlation of ACE exposures to poorer health outcomes ${ }^{1-4}$, unlike our results. Study limitations include small sample size, language barriers, disproportionate sample of sexes, and cultural differences unable to be detected by this research design. Nonetheless, the minimal childhood trauma reported in relation to health measures in these resource-poor Lima communities is unexpected and warrants further investigation. Noting the sensitive nature of the ACE survey, did the methods and clinical setting allow for greater reporting bias than other studies? Is there a factor which makes this dose-response relationship less potent in these regions? Is there a characteristic of Peruvian culture which is protective to the ACE effect? Acknowledging our sample did not capture the real population, an extensive gap in knowledge remains concerning ACEs impact among South American nations. Great emphasis should be placed on continuing this investigation into ACE relationships and health outcomes as the prevalence of chronic diseases continue to rise globally without paralleled increase in resources. As osteopathic physicians, striving to help our patients achieve self-healing and body unity, it is especially important to understand the complexity of underlying disease causation. Resource poor regions ignite an even greater need to optimize our clinical approach. Screening for ACEs may be a vital statistic to better understand our patients, promote a trusting relationship, and push for increased trauma-informed care training if only we had a more representative data sample to analyze.

\section{References}

1. Felitti VJ, Anda RF, Nordenberg D, et al. Relationship of childhood abuse and household dysfunction to many of the leading causes of death in adults. The Adverse Childhood Experiences (ACE) Study. Am J Prev Med. 1998;14(4):245-258. doi:10.1016/s07493797(9800017-8)

2. Huang $H$, Yan $P$, Shan Z, et al. Adverse childhood experiences and risk of type 2 diabetes: A systematic review and meta-analysis. Metabolism. 2015;64(11):1408-1418. doi:10.1016/j.metabol.2015. 08.0193.

3. Hughes K, Bellis MA, Hardcastle KA, et al. The effect of multiple adverse childhood experiences on health: a systematic review and meta-analysis. Lancet Public Health. 2017;2(8):e356-e366. doi:10. 1016/S2468-2667(1730118-4)

4. Liming, K.W., Grube, W.A. Wellbeing Outcomes for Children Exposed to Multiple Adverse Experiences in Early Childhood: A Systematic Review. Child Adolesc Soc Work J. 2018. 35, 317-335. https://doi.org/10.1007/s10560-018-0532-

Financial Disclosures: None reported.

Support: Acknowledgement: Statistician Alperen Korkmaz, MS \& Ohio University Heritage College of Osteopathic Medicine Seed Grant.

Seed grant funding covered costs of medical brigade supplies (finger-stick HbA1c equipment, personal protective 
equipment for clinic staff, copy and printing costs for survey distribution, etc.). Remaining funds were used to ameliorate travel costs of student researchers to and from Lima, Peru. Ethical Approval: This study was reviewed and approved by Ohio University Institutional Review Board IRB Protocol number: 17-X-88

Informed Consent: Patients could elect to participate after having a consent form verbally explained in Spanish by a native-speaker, in addition to being provided a written copy. Care was taken to ensure participants took time to familiarize themselves with the consent and understood survey completion and a finger-stick blood test would be involved before signing. If an adult could not provide written consent without outside intervention, such as a family member electing participation for them, said patient was excluded. Any patient $<18$ years old was also deemed ineligible.

Poster No.: ${ }^{\star} \mathrm{C} 25$

Abstract No.: 83

Category: Clinical

Research Focus Area: Chronic Diseases \& Conditions

\section{Spondylosis as a Potential} Musculoskeletal Manifestation of Ulcerative Colitis

${ }^{1}$ Ralfi Doka, OMS-IV; ${ }^{1}$ Landen "Shane" Burstiner, MS; ${ }^{1}$ Jake Herbert; ${ }^{1}$ Emily Teeter; ${ }^{2}$ Amor J. Royer, MD; ${ }^{2}$ Anna Owings, DO; ${ }^{3}$ Sarah Glover, DO; ${ }^{3}$ Pegah Hosseini-Carroll, MD

${ }^{1}$ Nova Southeastern University Dr. Kiran C. Patel College of Osteopathic Medicine (NSU-KPCOM); ${ }^{2}$ Department of Internal Medicine, University of Mississippi Medical Center; ${ }^{3}$ Department of Digestive Diseases, University of Mississippi Medical Center

Context: Musculoskeletal complaints are the most commonly seen extraintestinal manifestations (EIM) in inflammatory bowel disease (IBD) with a frequency up to $40 \%$; however, there is a continuous need to further explore this area. Literature has focused mainly in sacroiliatis and ankylosing spondylitis as axial manifestations of IBD; however, there is a lack of focus on other underlying axial complaints in IBD patients.

Objective: To determine if there is an underlying association between ulcerative colitis and the involvement of the axial skeletal system, mainly spondylosis.

Methods: This is a cross sectional study using a database of patients from 6 integrated healthcare facilities from 2011 to 2019. Data was collected from electronic health records of 3,104 patients diagnosed with IBD as determined by their ICD-10 codes (i.e., K50 or K51) and further subdivided based on the diagnosis of spondylosis as determined by the ICD-10 code M47. Our cohort was composed of African Americans and Caucasian patients. Statistics were calculated using t-test and chi-squared. A multivariate model was constructed for logistic regression.

Results: Our study had 228 patients with spondylosis diagnosed with an ICD-10 code, M-47. 5.8\% of our population with $\mathrm{CD}$ had comorbid spondylosis, compared to 9.4\% of our UC population $(\mathrm{p}<0.001)$. Multivariate modeling demonstrated UC was associated with an increased risk of spondylosis, even when controlling for age, race, sex, BMI, smoking status, and number of corticosteroid prescriptions (OR 1.64 [1.22-2.19] $\mathrm{p}=0.001$ ).

After conducting univariate analysis, female sex (OR $1.96[1.45-2.66] \mathrm{p}<0.001)$, BMI (OR 1.05 [1.03-1.06] $\mathrm{p}<0.001$ ), and diagnosis of UC (OR 1.58 [1.20-2.07] $\mathrm{p}=0.001)$ had positive associations with diagnosis of spondylosis. Age also had a positive association with diagnosis of spondylosis (OR 1.007 [1.0002-1.0139] $\mathrm{p}=0.045)$. It should be noted that the age has a positive correlation with UC, so the OR increases as the age increases. In order to better represent this association, we calculated the OR of the decade of life which resulted in a higher positive association (OR 1.073 [1.002 - 1.148] $\mathrm{p}=0.044$ ).

When looking at the combined MRI and X-Ray imaging of the spine $13.3 \% \mathrm{CD}$ patients and $17.6 \% \mathrm{UC}$ patients were imaged at our facilities. There was a statistical significance between these two findings $(\mathrm{p}=0.001)$ indicating that significantly more UC patients were subjected to imaging of the spine. When looking at these two imaging modalities separately, 6.1\% CD patients and 13.7\% UC patients had $\mathrm{X}$-Ray of the spine done at our facilities. On the other hand, 6.1\% of CD patients and 9.2\% patients obtained an MRI of the spine at our facilities. Even when stratifying for each imaging modality separately, the differences remained statistically significant for X-Ray and MRI of the spine, ( $\mathrm{p}=0.004$ and $\mathrm{p}=0.001$, respectively). There was no statistical significance when further subdividing the imagining of the spine in the cervical, thoracic, and lumbar regions. Conclusion: In our study we report spondylosis to be significantly more prevalent in Ulcerative Colitis, a relatively surprising finding. Given that spondylosis is not normally studied as a musculoskeletal manifestation of IBD, we were not able to find any reported association between IBD and spondylosis in the literature. It is well known that spondylosis is exacerbated due to inflammatory processes. Inflammation exacerbates the degeneration of the disc, endplate, fascia, and nerve tissue. We stipulate that the systemic inflammation of IBD could be a contributing factor to the association with spondylosis; however, the literature still lacks an explanation for why UC would 
have a significantly higher incidence of spondylosis than $\mathrm{CD}$. One prospective study showed that arthritis was the most common extraintestinal manifestation in CD (33\%) and UC (21\%). Another study reports that arthritis of peripheral or axial joints in IBD could be up to $40 \%$. While this could support the pathogenesis of joint degeneration due to systemic inflammation, our findings on spondylosis showed that it has a stronger association with UC.

To further characterize this association, we looked at how many patients had an image based diagnosis of spondylosis. While there was no significant association between $\mathrm{CD}$ and UC patients diagnosed with spondylosis via imaging, we did find that UC patients were subjected to significantly more imaging of the spine via X-Ray and MRI.

While our study could not define the underlying cause of this significant association, there could be an important link between UC and spondylosis, which has yet to be discovered. Future studies that include endoscopic evaluation of inflammation and radiographic characterization of the degree of spondylosis would be helpful to explore the connection between the inflammatory burden of IBD and the severity of spondylosis.

\section{References}

1. Wordsworth P. Arthritis and inflammatory bowel disease. Curr Rheumatol Rep. 2000 Apr;2(2):87-8. doi: 10.1007/s11926-0000045-3. PMID: 11123044.

2. Kolenkiewicz, M., Włodarczyk, A., \& Wojtkiewicz, J. (2018). Diagnosis and Incidence of Spondylosis and Cervical Disc Disorders in the University Clinical Hospital in Olsztyn, in Years 2011-2015. BioMed Research International, 2018, 5643839. https://doi.org/10.1155/2018/5643839

3. Adams, M. A., \& Roughley, P. J. (2006). What is intervertebral disc degeneration, and what causes it?. Spine, 31(18), 2151-2161. https://doi.org/10.1097/01.brs.0000231761.73859.2c

4. Ossum, A. M., Palm, Ø., Lunder, A. K., Cvancarova, M., Banitalebi, H., Negård, A., Høie, O., Henriksen, M., Moum, B. A., Høivik, M. L., \& IBSEN Study Group (2018). Ankylosing Spondylitis and Axial Spondyloarthritis in Patients With Long-term Inflammatory Bowel Disease: Results From 20 Years of Follow-up in the IBSEN Study. Journal of Crohn's \& colitis, 12(1), 96-104. https://doi. org/10.1093/ecco-jcc/jjx126

5. Shivashankar, R., Loftus, E. V., Jr, Tremaine, W. J., Harmsen, W. S., Zinsmeister, A. R., \& Matteson, E. L. (2013). Incidence of Spondyloarthropathy in patients with ulcerative colitis: a population-based study. The Journal of rheumatology, 40(7), 1153-1157. https://doi.org/10.3899/jrheum.121029

6. Middleton, K., \& Fish, D. E. (2009). Lumbar spondylosis: clinical presentation and treatment approaches. Current reviews in musculoskeletal medicine, 2(2), 94-104. https://doi.org/10. 1007/s12178-009-9051-
7. Buckwalter, J. A., Saltzman, C., \& Brown, T. (2004). The impact of osteoarthritis: implications for research. Clinical orthopaedics and related research, (427 Suppl), S6-S15. https://doi.org/10. 1097/01.blo.0000143938.30681.9d

8. Grant MP, Epure LM, Bokhari R, Roughley P, Antoniou J, Mwale F. Human cartilaginous endplate degeneration is induced by calcium and the extracellular calcium-sensing receptor in the intervertebral disc. Eur Cell Mater. 2016 Jul 25;32:137-51. doi: 10. 22203/ecm.v032a09. PMID: 27452962.

9. Li W, Gong Y, Liu J, Guo Y, Tang H, Qin S, Zhao Y, Wang S, Xu Z, Chen B. Peripheral and Central Pathological Mechanisms of Chronic Low Back Pain: A Narrative Review. J Pain Res. 2021 May 27;14:1483-1494. doi: 10.2147/JPR.S306280. PMID: 34079363; PMCID: PMC8166276.

10. Vavricka SR, Brun L, Ballabeni P, Pittet V, Prinz Vavricka BM, Zeitz J, Rogler G, Schoepfer AM. Frequency and risk factors for extraintestinal manifestations in the Swiss inflammatory bowel disease cohort. Am J Gastroenterol. 2011 Jan;106(1):110-9. doi: 10. 1038/ajg.2010.343. Epub 2010 Aug 31. PMID: 20808297.

11. Stephan R. Vavricka, MD, Alain Schoepfer, MD, Michael Scharl, MD, Peter L. Lakatos, MD, Alexander Navarini, MD, Gerhard Rogler, MD, Extraintestinal Manifestations of Inflammatory Bowel Disease, Inflammatory Bowel Diseases, Volume 21, Issue 8, 1 August 2015, Pages 1982-1992, https://doi.org/10.1097/ MIB. 0000000000000392

Financial Disclosures: None reported.

Support: None reported.

Ethical Approval: IRB File \#2019-0194

Study was reviewed and approved.

Informed Consent: N/A

Poster No.: ${ }^{\star} \mathrm{C} 26$

Abstract No.: 84

Category: Clinical

Research Focus Area: Osteopathic Philosophy

\section{A Pilot Study to Examine Medical Students' Perception of their Osteopathic Manipulative Therapy Education}

${ }^{1}$ Jessica Mazzi OMS-II; ${ }^{2}$ Nathan Leavitt; ${ }^{2} J e s s i c a$ Mazzi; ${ }^{2}$ Glen Kisby, PhD

${ }^{1}$ Western University of Health Sciences College of Osteopathic Medicine of the Pacific (WesternU/COMP); ${ }^{2}$ Department of Research, Western University of Health Sciences College of Osteopathic Medicine of the Pacific (WesternU/ COMP)

Context: Osteopathic manipulative therapy (OMT) is a practical outworking of the osteopathic principles and one of the foundations of an osteopathic physician's training. 
However, the utilization of OMT is on the decline. Previous studies have demonstrated a positive correlation between a medical student's propensity to utilize OMT in their future practice with both their OMT exposure prior medical school and the level of preclinical and clinical training that they received throughout medical school.

Objective: The goal of this pilot study was to assess how the OMT training curriculum influenced a medical student's perception of OMT and their intent to use the treatment modality as a future practicing physician.

Methods: A 13-question survey was created utilizing Qualtrics. The survey link was distributed through email by the clinical education department at two osteopathic medical schools to students in all four class years $(n=1320)$. The survey was open for one month. During this month, two additional reminder emails were sent; one at the twoweek mark and one on the last day of the survey. Results were collected through Qualtrics and analyzed with R.

Results: A response rate of $18.3 \%$ was collected for a sample distribution across year of Osteopathic Medical Student (OMS): OMS I (31.7\%), OMS II (21.3\%), OMS III $(24.6 \%)$ and OMS IV (22.5\%). Nearly half of all students (42.98\%) indicated that they were satisfied with their preclinical OMT training while $46.28 \%$ reported being "somewhat confident" in their ability to treat patients with OMT. The most notable positive influences on their perception of OMT were hands on experiences (41\%) and experiences prior to medical school (16.63\%). The most notable negative influences on a student's perception of OMT were clinical lecture hours $(31 \%)$ and virtual learning experiences (32\%). Overall, half of all students $(50 \%)$ had either a "greatly improved" or an "improved" perception of OMT through the course of their medical education, but the majority of students indicated that they would rarely $(21.6 \%)$ or never $(21.6 \%)$ use OMT in their future practice.

Conclusion: The most beneficial training modalities that had a positive effect on a student's perception of OMT were hands-on training and OMT exposure prior to medical school. In contrast, virtual lecture hours and preclinical lecture hours were perceived as negatively influencing a student's perception of OMT. These findings indicate that hands-on and real-world experiences, rather than passive learning modalities had the greatest impact on their perception of OMT. Despite these positive educational experiences, most students were only "somewhat confident" in their abilities, which is consistent with the majority of students indicating that they do not plan to routinely utilize their post-graduation OMT skills. Additional studies with a larger sample size and response rate will be required to determine the generalizability of our results with the goal of optimizing OMT education among Colleges of Osteopathic Medicine. Such optimization will ensure continued interest in and use of this valuable and underutilized treatment modality for patients.

\section{References}

1. Chamberlain NR, Yates HA. A prospective study of osteopathic medical students' attitudes toward use of osteopathic manipulative treatment in caring for patients. J Am Osteopath Assoc. 2003;103(10):470-478. doi:10.7556/jaoa.2003.103.10.470

2. Draper BB, Johnson JC, Fossum C, Chamberlain NR. Osteopathic Medical Students' Beliefs About Osteopathic Manipulative Treatment at 4 Colleges of Osteopathic Medicine. Med Educ.:16.

3. Pierce-Talsma S, Hiserote RM, Lund G. Osteopathic Medical Student Practice of Osteopathic Manipulative Treatment During School Break. J Am Osteopath Assoc. 2017;117(3):176-182. doi:10. 7556/jaoa.2017.033

4. Vazzana KM, Yao SC, Jung M-K, Terzella MJ. Perception-based effects of clinical exposure to osteopathic manipulative treatment on first- and second-year osteopathic medical students. J Am Osteopath Assoc. 2014;114(7):572-580. doi:10.7556/jaoa. 2014.111

5. R Core Team. 2019. R: A Language and Environment for Statistical Computing. Vienna, Austria: $R$ Foundation for Statistical Computing. http://www.R-project.org/.

Financial Disclosures: None.

Support: None.

Ethical Approval: Reviewed and approved by an Institutional Review Board with the following IRB number: 1721442-1.

Informed Consent: The informed consent was explicitly stated in the email that contained the survey link after listing the potential risks to the survey. Subjects by clicking the link to the survey consented to being a part of the study.

Poster No.: ${ }^{\star} \mathrm{C} 27$

Abstract No.: 85

Category: Clinical

Research Focus Area: Chronic Diseases \& Conditions Effects of face masks on oxygen saturation at graded exercise interval

Clinnt Luna Favo OMS-III; ${ }^{2}$ Varnita Vishwanath; ${ }^{3}$ Blake Anderson 
Midwestern University Arizona College of Osteopathic Medicine (MWU/AZCOM)

Context: During the progression of the Covid-19 pandemic, most states across the U.S.A. mandated/recommended mask wearing to slow the spread of the virus. With the increased use of various mainstream (cloth) and medical face masks (surgical and N95) available to the public, there is potential public concern in regard to how face masks may affect our breathing and cardiopulmonary health. Such non-evidence based intrepidations may limit use of mask wearing in the general population.

Objective: To assess heart function (via heart rate) and lung function (via pulsoximetry) among healthy adults exposed to various types of face masks under conditions of rest and graded exercise. We aimed to provide evidence that mask wearing does not influence cardio-respiratory function in healthy individuals.

Methods: We performed a randomized controlled study where we evaluated male $(n=20)$ and female $(n=20)$ healthy subjects that met our inclusion criteria (between the ages of 21-65 years old). Subjects with underlying medical conditions such as any respiratory and cardiovascular illness, or any other illness that may affect the ability to conduct any activity required of the study were excluded. We recruited participants that were faculty, staff, and students from Midwestern University. All experiments were performed on university campus grounds abiding by school and CDC guidelines on social distancing. This is a cross-sectional study where physiological parameters were measured while subjects participated in three activity levels (duration of 10 minutes each) in a randomly assigned order: no intensity/rest (sitting), moderate intensity (walking), and high intensity (climbing stairs). Each subject served as their own control. The subject's age and BMI were collected prior to the start of the activity. Subjects conducted every activity level wearing every face mask condition separately: no mask, surgical mask, and N95 respirator; and therefore, each subject performed 9 activities. Heart rate (HR) and blood oxygen saturation levels (SpO2) via pulse oximeter were collected after every activity level. Perceived exertion level was assessed utilizing the Borg-15-point scale. We performed a one-way ANOVA to compare the results of the Borg scale, HR, and $\mathrm{O} 2$ saturation between each activity level for the same mask variation. The study was approved by MWU-IRB (AZ\#1422).

Results: There were 20 males (aged $28.35 \pm 1.98$ ) and 20 females (aged $29.10 \pm 2.45$ ) in our study. We found no statistically significant difference in SpO2 levels at either exercise or rest between the no mask, surgical mask, and
N95 mask group. Additionally, we performed a one-way ANOVA for the results of the $\mathrm{SpO} 2$ between each mask variation for the same activity level. The means for $\mathrm{SpO} 2$ at rest was compared between no mask, surgical mask and N95. No differences noted between the groups $(\mathrm{P}>0.05$, one-way ANOVA). Our study did, however, find a statistically significant difference in $\mathrm{SpO} 2$ at rest between male and female subjects within every mask variation. The $\mathrm{SpO} 2$ levels were on average decreased by 1.6\% (no mask), $1.4 \%$ (surgical mask), 1.7\% (N95 mask) in males compared to female counterparts at rest. We found this to inversely correlate with BMI such that the male subjects (average $\mathrm{BMI}=27.79 \pm$ 0.98), had consistently lower SpO2 levels at rest in comparison to female subjects (average BMI $=24.01 \pm 0.69$ ). Our study also found a statistically significant difference in perceived exertion between each successive exercise level. This was directly correlated with an increase in heart rate with graded exercise.

Conclusion: Our results indicate that face masks (both surgical and N95) do not influence either heart rate or blood oxygen levels, providing evidence of adequate cardiorespiratory function while wearing masks with concomitant physical activity. Our data provides evidence to quell fears that mask wearing adversely affects heart and respiratory function in healthy individuals. Future studies evaluating face masks and blood oxygen levels in those with underlying medical conditions may further provide insight on effects of face masks on cardio-respiratory health.

\section{References}

1. Center for Disease Control and Prevention. CDC calls on Americans to wear masks to prevent COVID-19 spread. CDC Newsroom website. Published July 14, 2020. Accessed March 17, 2021. https://www.cdc.gov/media/releases/2020/p0714-americans-towear-masks.html.

2. Joo H, Miller GF, Sunshine G, et al. Decline in COVID-19 hospitalization growth rates associated with statewide mask mandates 10 states, March-October 2020. MMWR Morb Mortal Wkly Rep. 2021;70(6):212-216. https://doi.org/10.15585.mmwr.mm7006e2.

3. Liang $M$, Gao L, Cheng $C$, et al. Efficacy of face mask in preventing respiratory virus transmission: A systematic review and metaanalysis. Travel Med Infect Dis. 2020;36:101751. doi: 10.1016/j. tmaid.2020.101751.

4. Batazy A, Toivola M, Adhikari A, Sivasubramani SK, Reponen T, Grinshpun SA. Do N95 respirators provide 95\% protection level against airborne viruses, and how adequate are surgical masks? Am J Infect Control. 2006;34(2):51-7. doi: 10.1016/j.ajic.2005.08.018.

5. The White House. Executive order on protecting the federal workforce and requiring mask-wearing. The White House Briefing Room 
website. Published January 21, 2021. Accessed March 17, 2021. https://www.whitehouse.gov/briefing-room/presidentialactions/2021/01/20/executive-order-protecting-the-federalworkforce-and-requiring-mask-wearing/.

6. Markowitz, A. State-by-State guide to face mask requirements. AARP Health website. Published March 16, 2021. Accessed March 17, 2021. https://www.aarp.org/health/healthy-living/info-2020/ states-mask-mandates-coronavirus.html.

7. University of Washington. Maps of mask use. IMHE website. Published January 20, 2021 Accessed March 17, 2021. http:// www.healthdata.org/acting-data/maps-mask-use.

8. Kreuter F, Barkay N, Bilinski A, et al. (2020). Partnering with a global platform to inform research and public policy making. Surv Res Methods. 2020;14(2):159-63. https://doi.org/10.18148/ srm/2020.v14i2.7761.

9. Social Data Science Center. Global trends of mask usage in 19 million adults. University of Maryland. Social Science Data Center website. Published October 12, 2020. Accessed March 17, 2021. https://socialdatascience.umd.edu/global-trends-of-maskusage-in-19-million-adults/.

10. Epstein D, Korytny A, Isenberg Y, et al. Return to training in the COVID-19 era: The physiological effects of face masks during exercise. Scand J Med Sci Sports. 2021;31:70-75. https://doi. org/10.1111/sms.13832.

11. Centers for Disease Control and Prevention. Perceived exertion (Borg rating of perceived exertion scale). CDC website. Published September 17, 2020. Accessed March 17, 2021. https://www.cdc. gov/physicalactivity/basics/measuring/exertion.htm.

12. Rating of perceived exertion: Borg scales. Heart Online website. Updated November 2014. Accessed September 4, 2020. https:// www.sralab.org/sites/default/files/2018-04/Rating_of perceived_exertion_-_Borg_scale.pdf.

Financial Disclosures: None reported.

Support: None reported.

Ethical Approval: MWU-IRB (AZ\#1422)

Informed Consent: Consent forms were attained in addition to the school's COVID-19 health questionnaire upon admission. The health assessment was reported as a selfassessment of each subject.

Poster No.: ${ }^{\star} \mathrm{C} 28$

Abstract No.: 87

Category: Clinical

Research Focus Area: Chronic Diseases \& Conditions

Prioritizing Preventative Health in Older Adults Residing in Long-Term Care Facilities Through Vaccination

${ }^{1}$ Fatima Maqsood, OMS-III; ${ }^{1}$ Margaret M. McGrath, OMS-III; ${ }^{1}$ Sandra Rabat, OMS-III; ${ }^{2}$ Kate Whelihan, MPH CPH

${ }^{1}$ A.T. Still University, School of Osteopathic Medicine in Arizona (ATSU-SOMA); ${ }^{2}$ Department of Public Health, A.T.
Still University, School of Osteopathic Medicine in Arizona (ATSU-SOMA)

Context: Long-term care facilities (LTCFs) house adults who require acute rehabilitation or long-term high-level care. Influenza and pneumonia cause $90 \%$ of deaths in adults $65+$; only $42-66 \%$ of Pennsylvania LTCF residents receive these vaccinations. ${ }^{1}$ Given the COVID-19 pandemic, it is crucial to bring awareness to gaps in vaccination of LTCF residents in order to promote the overall health of vulnerable populations and limit the spread of disease.

Objective: To identify the perceived barriers to the vaccination of residents in LCTFs across Pennsylvania.

Methods: LTCFs located in Pennsylvania, identified by zip-code, were contacted via publicly available contact information and administrators were asked to participate in an anonymous phone survey designed to gather information regarding vaccination practices. A total of $22 \mathrm{LTCF}$ agreed to participate. Our survey contained questions and discussion prompts designed to assess the following: how vaccines are recorded, what information is recorded, how charts are checked for vaccination gaps, the vaccination process, and perceived barriers to vaccination. Analysis of individual LTCFs consisted of a basic descriptive analysis of the aggregated data.

Results: Of 406 eligible LTCFs, 109 were contacted and 22 agreed to participate (20.2\%). Thirteen centers reported use of electronic records; 7 use both paper and electronic records. Vaccine records reportedly included patient identifying information (95.2\%), injection site (76.1\%), injection information (38.1\%), and vaccination history (90.5\%). Methods for vaccine tracking included chart reviews (28.6\%), electronic alerts (42.9\%), scheduled audits (38.1\%), and vaccination at admission (19\%). The most common vaccination process involved a physician ordering and administered by a nurse (57.1\%). The main barriers perceived by more than half of participants included lack of patient education and issues with vaccine acquisition.

Conclusion: Although vaccination is vital to preventative health, $52.4 \%$ of LTCFs perceived barriers in vaccinating their residents. We determined a baseline understanding of how LTCFs track vaccination status and perceived barriers they face in getting residents vaccinated. Individual LTCFs can examine their own system to understand gaps they may face in vaccinations. Given the importance of vaccination in bringing an end to the COVID-19 pandemic, recognizing potential impediments to achieving higher vaccination rates in such a vulnerable population is essential. Future research projects could look at steps that 
can be taken to overcome these barriers and ultimately increase vaccination rates.

\section{References}

1. Pennsylvania Patient Safety Advisory. Increasing Influenza and Pneumonia Vaccination Rates in Long-Term Care: Advisory.Pennsylvania Patient Safety Authority, 2013

Financial Disclosures: None reported.

Support: None reported.

Ethical Approval: The ATSU-AZ IRB reviewed the application titled "Prioritizing Preventative Health in Older Adults Residing in Long Term Care Facilities Through Vaccination.” The study was deemed exempt by the IRB.

IRB Determination: Exempt Protocol \#2020-263 (minimal risk)

Informed Consent: Identified facilities were contacted via phone for an initial assessment of willingness to participate and gain of verbal consent to participate in the survey. Contacted facilities were told of the purpose and aim of our study. Facilities that were contacted were offered to have a study information document sent to them outlining our study prior to obtaining consent. Verbal consent was obtained over the phone prior to proceeding with the survey.

Poster No.: *C29

Abstract No.: 89

Category: Clinical

Research Focus Area: Acute and Chronic Pain Management

\section{Hispanic and Non-Hispanic Atti- tudes Towards Total Joint Arthroplasty}

${ }^{1}$ Yesenia Anaya PhD, OMS-II; ${ }^{2}$ Jaydee Foster, MA; ${ }^{3} \mathrm{Scott}$ Smith, PhD; ${ }^{2}$ Roberto J Fajardo

${ }^{1}$ University of the Incarnate Word School of Osteopathic Medicine (UIWSOM); ${ }^{2}$ Department of Clinical and Applied Science Education, University of the Incarnate Word School of Osteopathic Medicine (UIWSOM); ${ }^{3}$ Department of Mathematics and Statistics, University of the Incarnate Word School of Osteopathic Medicine (UIWSOM)

Context: The Hispanic population in the US utilizes TKA approximately $30 \%$ less when compared to non-Hispanic Caucasians (NHC) despite a similar incidence of OA. This discrepancy persists even when socioeconomic factors (e.g., health insurance availability) are controlled for in statistical analyses. The social, cultural, and socioeconomic factors underlying underutilization are not well studied and require further investigation to better understand patters of underutilization.

Objective: To identify cultural, social, and socioeconomic factors that influence a person's decision to pursue medical attention or to undergo a surgical joint replacement procedure due to osteoarthritis (OA).

Methods: We used a cross sectional survey study design. Subjects were recruited in person at community events/ centers, farmers markets, vaccination drives, and other public gatherings on the south side of San Antonio. This is a federally designated medically underserved region of Bexar County, Texas. Eligible subjects were between the ages of 50 and 89, residents of Bexar County, and fluent in English. All responses were anonymous, and no identifiers were collected. Surveys consisted of a combination of specific questions and Likert scale questions to determine an individual's socioeconomic status, pain tolerance, health literacy, family support conditions, and other factors that influence a person's decision to seek medical treatment. Nonparametric and polychoric correlation analyses were used to compare responses from Hispanic and nonHispanic Caucasians (NHC) and the associations between survey questions/prompts. Although this work ultimately relates to end-stage arthritis, it examines one step in the natural history of osteoarthritis and thus incorporates aspects of the biomechanical and behavioral osteopathic models.

Results: $60 \%$ of the 233 subjects were female. Female and male mean ages were $65.8( \pm 9.0)$ and $64.3( \pm 8.8)$, respectively. Hispanic subjects comprised $63 \%$ of the sample. More than $80 \%$ of respondents reported having private, military, Medicare, or Medicaid health insurance plans. Among all respondents that reported daily knee or hip pain, approximately 50\% indicated they would avoid a total joint arthroplasty (TJA) due to cost even though it could alleviate joint pain. Polychoric correlations indicated a significant relationship between those who reported a lower household income and an avoidance of surgery due to fear. Furthermore, respondents who indicated that enduring long-term pain was part of God's plan reported having an above average pain tolerance. Compared to NHW, Hispanics were more likely to report fear of TJA ( $p=0.001$ ) and being influenced by another's surgical experience $(p=0.045)$. Within women, Hispanic females were more likely to report fear of TJA than NHC females 
$(\mathrm{p}=0.002)$. Compared to Non-Hispanic females, Hispanic females were more likely to choose a doctor based on their cultural heritage $(\mathrm{p}=0.034)$ and indicated a belief that pain is part of God's plan ( $\mathrm{p}=0.000)$. Within Hispanics, females did not like to ask for help $(\mathrm{p}=0.001)$ and reported that they don't want their health to affect others ( $\mathrm{p}=0.016)$.

Conclusion: These results begin to reveal several possible personal and cultural attitudes that may underly underutilization trends by Hispanic patients. First, Hispanic respondents indicated a greater fear of a TJA procedure than NHC counterparts. Also, Hispanic respondents indicated that the surgical experience of another would influence his/her decision to undergo surgery. Finally, our data suggest that some Hispanic individuals see enduring longterm pain as fate or destiny associated with their religious beliefs. This preliminary work may provide us some insight into the multifactorial contributions to Hispanic TJA underutilization. This is a critical first step towards developing strategies to mitigate underutilization and thus, reduce the number of patients that endure arthritic pain when a pain-reducing treatment is available.

\section{References}

1. Stone Andrea, MacDonald James, Joshi Maulik, King Paul. 2019. Differences in Perioperative Outcomes and Complications Between African American and White Patients After Total Joint Arthroplasty. J Arthroplasty

2. Venugopal Vivek, Gronbeck Christian, Harvey Lucas, Patel Aalok, Harrington Melvyn, Halawi Mohamad. 2020. Time Trends in Perioperative Characteristics and Health Outcomes in Hispanic Patients Undergoing Primary Total Knee Arthroplasty. J Racial Ethnic Health Disp.

3. Cusano A, Venugopal V, Gronbeck C, Harrington M, Halawi M. September 17, 2020. Where Do We Stand Today on Racial and Ethnic Health Inequalities? Analysis of Primary Total Knee Arthroplasty from a 2011-2017 National Database. J Racial Ethnic Health Disp

4. Conner-Spady Barbera, Bohm Eric, Loucks Lynda, Dunbar Michael, Marshal Deborah, Noseworthy Tom. 2020. Patient expectations and satisfaction 6 and 12 months following total hip and knee replacement. Qual Life Res.

Financial Disclosures: None reported

Support: RJF received a faculty seed grant for this study from the Office of Research and Innovation at the University of the Incarnate Word School of Osteopathic Medicine. Ethical Approval: This exempt study is approved by the UIW institutional review board (IRB) (\#19-03-002).

Informed Consent: Verbal informed consent was obtained from all subjects prior to completing a survey.
Research team members used a script to ensure that descriptions of the study were accurate and uniform. Private/ identifiable information was not collected during by the survey instrument. After providing verbal consent, each subject received a copy of the survey face page that included a description of the study and detailed the contact information for the PI, research coordinator, and contacts at IRB office.

Poster No.: ${ }^{\star} \mathrm{H} 1$

Abstract No.: 8

Category: Health Services

Research Focus Area: Osteopathic Philosophy

\section{Helping Individuals Learn of Their Federal Right to Read Their Health Record with a YouTube Community Outreach Message}

${ }^{1}$ Shalaya Asal Yazdi, OMS-I; ${ }^{2}$ Susan St. Pierre, DO; ${ }^{3}$ Glenn Davis, MS; ${ }^{4}$ Michael Warner, DO, CPC, CPCO, CPMA, AAPC Fellow

${ }^{1}$ Touro University College of Osteopathic Medicine-CA (TUCOM); ${ }^{2}$ Department of Primary Care, Touro University College of Osteopathic Medicine-CA (TUCOM); ${ }^{3}$ Department of Academic Affairs, Touro University College of Osteopathic Medicine-CA (TUCOM); ${ }^{4}$ College of Osteopathic Medicine, Touro University College of Osteopathic Medicine-CA (TUCOM)

Context: Individuals have a federal right to read their health records. Why it is important to read your health record and how it needs to be requested may not be common knowledge. Benefits of reading your health record after a medical encounter gives you a chance to check for errors or omissions and also assess how your story is represented. This project created a community outreach video, posted it on YouTube and assessed viewer responses with an IRB approved survey.

Objective: To measure the effect of a community outreach message educating the public of the importance of reading their health record and how to gain access to health records in accordance with the law. This message also stressed the importance of reading the entire health record, including the History, Examination, and Medical Decision Making. The hypothesis of this community outreach message believes informed patients can make better advocates for themselves when engaging in health care services. 
Methods: An 18:45-minute YouTube video was created to raise awareness of the new federal medical documentation guidelines.

A script was written prior to the recording to assure that information delivered was accurate and concisely delivered in an 18:45-minute episode. The script was reviewed by a certified professional coder/medical auditor/physician to assure accuracy when describing new federal policies rights conferred by law. Cast members were recruited and given the opportunity to review and rehearse the script before the recording.

The video was recorded from a Zoom meeting with 14 participants, which included Osteopathic Medical Students, Master of Science students, a faculty member and a founder and Community Outreach Coordinator of the IgA Nephropathy Foundation of America. Participants were asked to join a Zoom meeting on Monday, October 12, 2020 05:29 PM Pacific Time (US and Canada). Participants were informed The Patient Advocacy Video will be recorded and signed general release waivers granting our research team permission to share the community outreach video. The video was approved by the Provost's Office with disclaimers posted in the description and verbalized during the video introduction.

An IRB approved Qualtrics Anonymous Survey was created. The Qualtrics Anonymous Survey was offered in the YouTube Comment section after the video was posted on YouTube. Informed consent was obtained from all participants via Qualtrics survey.

Results: 19 surveys were completed with $100 \%$ of participants consenting to protocols of the IRB approved study [IRB M-2120]. When asked if viewers were aware of federal rights to access or read their health record, $25 \%$ knew of this federal right, $37.5 \%$ knew of the right, but not the details, and 37.5\% learned about this right with the YouTube video community outreach message. 32\% reported being curious about what was written about them in their health record. 50\% had requested to access or read their health records. Of those who had requested access to their health care records, $57 \%$ were actually able to do so, $0 \%$ were told they did not have permission, and $43 \%$ did not receive any feedback from a request. Of those who read their story in their health record: $20 \%$ reported very accurate, $40 \%$ reported mostly accurate, 40\% reported accurate, but not complete, $0 \%$ reported not accurate. When asked if participants knew of their federal right to amend/co-author their health record, $7 \%$ knew of the right, 20\% knew, but not the details, and 73\% learned by viewing the community outreach video. $29 \%$ identified themselves as a medical professional or training to enter the medical profession. Viewers commented appreciation for the knowledge and requested more educational videos.

Conclusion: Medical records started as a means for health care providers to document findings from medical health encounters. Studies have shown that the use of electronic health record documentation has been associated with physician burnout and reduction of professional satisfaction, which can impact the accuracy of the medical health record. Accurate and thorough medical health records are critical elements for successful patient care, risk management, and liability prevention. Individuals have a federal right to access and read their medical health records. Benefits of reading the health record after a medical encounter gives a patient a chance to review for errors or omissions.

According to this study, most viewers have limited knowledge of their federal right to access or read their records; such rights may impact all individuals seeking healthcare. People are curious about what is written in their health records, it's important to be able to access the health records and ensure accuracy.

The objective of this study was to measure the effect of a community outreach message educating the public of the importance of reading their medical health record. In addition, this study had provided information to viewers regarding how to gain access to health records in accordance with the law. We believe this type of engagement with the public has the potential to educate why it is important to read the entire health record and how it can be done in accordance with federal law. Additional studies can investigate the implications of inaccurate medical health records.

\section{References}

1. 1995 Documentation Guidelines for Evaluation and Management Services, CMS.gov, Medicare Physician Guide. https://www.cms. gov/Outreach-and-Education/MedicareLearning-Network-MLN/ MLNEdWebGuide/Downloads/95Docguidelines.pdf 2.

2. 1997 Documentation Guidelines, CMS.gov, Medicare Physician Guide. https://www.cms.gov/Outreach-and-Education/MedicareLearning-NetworkMLN/MLNEdWebGuide/Downloads/ 97Docguidelines.pdf

3. Valikodath NG, Newman-Casey PA, Lee PP, Musch DC, Niziol LM, Woodward MA. Agreement of Ocular Symptom Reporting Between Patient-Reported Outcomes and Medical Records. JAMA 
Ophthalmol. 2017;135(3):225-231. doi:10.1001/jamaophthalmol. 2016.5551

4. Berdahl CT, Moran GJ, McBride O, Santini AM, Verzhbinsky IA, Schriger DL. Concordance Between Electronic Clinical Documentation and Physicians' Observed Behavior. JAMA Netw Open. 2019;2(9): e1911390. Published 2019 Sep 4. doi:10.1001/ jamanetworkopen.2019.11390

5. Warner M, St. Pierre S, Davis G, Bains R, Gill A, Singh J, Antos A, Lim S, Molga H, Malik A, Masek M, Prasad P, Yazdi S. Touro University California Documentation Guidelines- a guide for medical students in light of drastic 2021 health record documentation policy changes. 2020. http://patientadvocacyinitiatives.org/Portals/0/ TUC-DG\%201sept2020.pdf

6. Warner M. 2018. When a Patient Requests Access to their Medical Record. Healthcare Business Monthly. https://www.aapc.com/blog/ 40514-when-a-patient-requests-access-to-their-medical-record/

7. Street RL Jr, Liu L, Farber NJ, et al. Provider interaction with the electronic health record: the effects on patient-centered communication in medical encounters. Patient Educ Couns. 2014;96(3):315-319. doi:10.1016/j.pec.2014.05.004

8. "How Doctors Feel About Electronic Health Records; National Physician Poll by The Harris Poll." Stanford Medicine EHR National Symposium, 4 June 2018, med.stanford.edu/ehr/electronic-health-records-poll-results.html.

9. Wachter, R "The Digital Doctor: Hope, Hype, and Harm at the Dawn of Medicine's Computer Age," 2017 The Digital Doctor: Hope, Hype, and Harm at the Dawn of Medicine's Computer Age

10. Gutheil TG. Fundamentals of medical record documentation. Psychiatry (Edgmont). 2004;1(3):26-28.

11. Ehrenfeld JM, Wanderer JP. Technology as friend or foe? Do electronic health records increase burnout?. Curr Opin Anaesthesiol. 2018;31(3):357-360. doi:10.1097/AC0.0000000000000588 [https://pubmed.ncbi.nlm.nih.gov/29474217/]

Financial Disclosures: None reported.

Support: None reported.

Ethical Approval: This study has IRB approval [IRB M-2120], granted by the Touro University California Internal Review Board. Researchers have CITI Certifications.

Informed Consent: An IRB approved Qualtrics Anonymous Survey was created. The Qualtrics Anonymous Survey was offered in the YouTube Comment section after the community outreach video was posted on YouTube. Informed consent was obtained from all participants via Qualtrics survey. The informed consent was asked as follows: "I consent to answering this survey knowing my responses are anonymous in accordance with Touro University California's Institutional Review Board (IRB).

(Skip logic: Yes -> \#2, No -> end of survey)”

\footnotetext{
${ }^{\star}$ Poster No.: ${ }^{\star} \mathrm{H} 2$

Abstract No.: 37

Category: Health Services
}

Research Focus Area: Chronic Diseases \& Conditions

\section{Early Detection of Lung Cancer in Adults: Quality Improvement and Implementation in the Rural Health Setting}

${ }^{1}$ Jonathan Carbungco MS, OMS IV; ${ }^{1}$ Brittany Woody, MS; ${ }^{1}$ Britni Smith, DO; ${ }^{2}$ Veronica Hill, MSN; ${ }^{2}$ Robin Fischer, DNP; ${ }^{2}$ Justin Hovey, MD

${ }^{1}$ Alabama College of Osteopathic Medicine (ACOM); ${ }^{2}$ Ashford Clinic, Alabama College of Osteopathic Medicine (ACOM)

Context: Lung and bronchus cancers represented the highest incidence of cancer related deaths in the United States, and the third highest incidence of new cancers, these rates are even higher in Alabama. In 2013, the USPSTF issued a recommendation advising all patients between age 55-80 years old with a 30-pack-year or greater smoking history who smoked in the last 15 years to receive annual Low Dose CT scan. The goal of LDCT is to identify malignancy early to improve prognosis and treatment efficacy.

Objective: Rural clinics face many difficulties implementing screening guidelines compared to their urban counterparts. Cultural, educational, socioeconomic, and resource barriers pose challenges both to providers and patients and require rural clinics to find creative and efficient solutions to provide the care patients require. These problems prompted the ACOM Ashford Clinic to implement a QI study to identify patients meeting the 2013 USPSTF, assess, and improve the rates of low dose CT scans.

Methods:

Method: The data was collected using a quasi-experimental study design.

Participants: A report from the EMR including patients age 55-77 with a smoking history yielded 273 patients. $\mathrm{Pa}-$ tients were called by phone to collect detailed smoking history. Individuals who did not meet LDCT criteria or declined participation were excluded. 84 eligible patients were identified and appropriately consented for participation. Statistical analyses of the participants showed no significant difference in sex, age, pack years, or current smoking status.

Intervention and Data Collection: A standardized script for calling the eligible patients. After exclusion, participants' next appointment date was gathered that clinic staff could 
reference to provide intervention at that time. A tobacco dependence syndrome identifier was added to the patient's chart with smoking history details, allowing clinicians to calculate pack years per patient. Clinicians were given decision-making tools for the appointment to provide guidance on LDCT and note patient eligibility. Patients were referred for LDCT if desired. At the end of the implementation period, participant records were examined to note LDCT compliance.

Statistical Analysis: Demographic statistics including sex, age, pack years, and current/noncurrent smokers were recorded before and after analysis of participants. The chisquare and Mann-Whitney U tests showed no significant differences in these population comparisons. A MannWhitney $\mathrm{U}$ test was run to compare the rate of compliance in ordering LDCTs before and after implementation.

Osteopathic Significance: The objective of this study promote the Osteopathic Tenets by providing rational, evidence-based care to all patients, acknowledging the body as a unit, promoting its ability to self-regulate, and understanding the structure-function relationship and educating patients on how these affect their state of health. Results: After implementation, 55 of 84 patients were compliant with LDCT guidelines, yielding a compliance rate of $65.5 \%$. Twenty-one participants who were not compliant before the study became compliant after implementation and six patients who were compliant prior to the study became noncompliant by the end. Two of six patients who were compliant before but not after the study were not seen in the clinic after the study began. Of the 25 patients who were noncompliant before and after implementation, five were not seen at the clinic after the study began. The two patients who initiated care during the study and were eligible for LDCT were compliant. Two patients were considered compliant despite deciding not to receive an LDCT as shared decision making was employed.

A compliance rate of $46.3 \%$ before implementation, a rate of $65.5 \%$ after implementation, and a sample size of 82 and 84 patients respectively yielded a Z-score of 2.5292 with a p-value of 0.0057 . There was an overall 19.2\% increase in compliance rate. The relative uplift in compliance rate (change divided by initial rate) is $41.29 \%$. The observed power is $88.86 \%$. The standard error of the before and after rates are 0.055 and 0.052 , respectively. The standard error of difference is 0.076 .

Conclusion: The changes implemented during this study were seen to have significantly increased the clinic's compliance with the USPSTF lung cancer screening guidelines. Improving a provider's awareness of LDCT guidelines and providing a tangible document to share with patients improved the rate of compliance. Additionally, providing literature to patients and having a brief discussion of the recommendations might have decreased the number of patients who declined an LDCT despite being eligible. Only two of 84 patients (2.4\%) denied an LDCT when they were offered one by a provider.

References None reported.

Financial Disclosures: None reported.

Support: None reported.

Ethical Approval: This study was approved by the Alabama College of Osteopathic Medicine IRB (Number HS201217-EX) and meets the criteria for Expedited Category 7.

Informed Consent: Informed consent was obtained by all participants by standardized phone call.

^Poster No.: ${ }^{\star} \mathrm{H} 3$
Abstract No.: 47
Category: Health Services
Research Focus Area: Osteopathic Philosophy
High school students` perceptions
of careers in healthcare and
awareness of the osteopathic
profession increase by
participation in a healthcare camp

${ }^{1}$ Ashley Lynn Orr MS, OMS-II; ${ }^{2}$ Kasey M. Kruse; ${ }^{2}$ Joseph M. Vroegop; ${ }^{2}$ Erica L. Ausel, PhD; ${ }^{3}$ David C. Eland, DO; ${ }^{3}$ John C. Turner, PhD; ${ }^{4}$ Clint L. Whitson, MS; ${ }^{5}$ Brian W. Skinner, PharmD, BCPS; ${ }^{2} J u l i a$ M. Hum, PhD

${ }^{1}$ Marian University College of Osteopathic Medicine (MU-COM); ${ }^{2}$ Division of Biomedical Sciences, Marian University College of Osteopathic Medicine; ${ }^{3}$ Division of Clinical Sciences, Marian University College of Osteopathic Medicine; ${ }^{4}$ Office of Student Affairs, Marian University College of Osteopathic Medicine; ${ }^{5}$ Division of Clinical Sciences, Marian University College of Osteopathic Medicine;

Context: Efforts to increase the diversity among healthcare providers may help close the gap of health disparities, combat inequities, and address medical access in underserved populations ${ }^{1}$. In response to the need for more healthcare providers from populations under-represented in medicine, Marian University College of Osteopathic Medicine (MU-COM) hosted a week-long day camp for high school students with the goal of introducing them to different healthcare careers and osteopathy. 
Objective: To determine the effect of a healthcare camp on recognition of careers in medicine and awareness of the osteopathic profession.

Methods: The hypothesis was tested through administration of a voluntary survey pre- and post-camp. All 26 campers that attended the healthcare camp participated in the voluntary survey. To maintain anonymity, each participant generated a unique code used for both pre-and post-camp surveys to facilitate matched response data analysis. In addition to asking participants to list careers in healthcare during the pre-camp survey, demographic questions were collected. Demographic data included gender, race, ethnicity, the presence of a healthcare worker in their immediate family, and previous participation in a healthcare camp. Campers rotated through activity stations daily, highlighting a unique patient case using the Simulation Center, Anatomage table, ShareCare virtual reality system, and osteopathic manipulative medicine (OMM) lab. Further, onboarding and debriefing sessions were utilized to facilitate discussion and contextualize relative healthcare provider roles related to the patient case of the day. Current osteopathic medical students, along with other students in the health professions, served as counselors and activity leaders allowing campers to create connections with in-training members of the medical community. At the conclusion of the healthcare camp, a second survey was administered to all participants that asked campers to list careers in healthcare. A Wilcoxan Signed-Rank test was employed to compare participants' pre- and post-camp responses of their perceptions of healthcare careers. The effect of demographic data on responses to careers in healthcare were analyzed using either the Mann-Whitney U test or the Kruskal-Wallis ANOVA, as appropriate.

Results: The response rate for both pre- and post-camp surveys was $100 \%$ with $15 \%$ of participants identifying as male and $85 \%$ as female. The participants included three sophomores (11.5\%), nine juniors (34.6\%), twelve seniors (46.2\%), and two incoming college freshmen (7.7\%). Additionally, 24\% of participants indicated their ethnicity as Hispanic while the remainder selected Non-Hispanic. Of the campers $46.1 \%$ identified as Caucasian, $42.3 \%$ as Black, and $15.3 \%$ preferred not to answer. Prior to the intervention of the healthcare camp, participants were able to list on average 5.4 healthcare professions, which significantly increased to 11.5 careers on the post-camp survey $(\mathrm{p}<0.05)$. Awareness of the osteopathic profession, as indicated by listing DO, osteopathy, osteopathic, or OMM in the pre- and post-camp surveys, increased from 0 to $42 \%$. Demographic data did not significantly affect program outcomes as assessed by survey data related to perception of careers in healthcare or awareness of the osteopathic profession.

Conclusion: The survey results support the implementation of a healthcare camp as an effective measure to increase the perception of careers in healthcare while also enhancing the awareness of the osteopathic profession in high school students. Future healthcare camps will include an overnight component to expand the student population beyond that of central Indiana. Further, as a means of encouraging and supporting campers to continue to pursue a career in healthcare, camp attendees are eligible for additional scholarships should they attend Marian University.

\section{References}

1. Marrast LM, Zallman L, Woolhandler S, Bor DH, McCormick D. Minority physicians' role in the care of underserved patients: diversifying the physician workforce may be key in addressing health disparities. JAMA Intern Med. 2014; 174(2): 289-291. doi: 10.1001/ jamainternmed.2013.12756

Financial Disclosures: None reported.

Support: Support for the Marian University Healthcare Camp was provided through a generous donation from the Julie and Tom Wood Family Foundation. The survey distribution and completion was not financially supported.

Ethical Approval: This study was submitted to the Marian University Institutional Review and received IRB approval (IRB \#S21.283).

Informed Consent: Parental consent was obtained for each participant, as well as assent forms for each camper were signed.

Poster No.: * ${ }^{\star} 4$

Abstract No.: 60

Category: Health Services

Research Focus Area: Chronic Diseases \& Conditions

The Incidence of Cancer Diagnoses in the Rural Southwest: A 16-Year Retrospective Analysis

${ }^{1}$ Phillip Hasenbalg, OMS-IV; ${ }^{2}$ Diana Lalitsasivimol, PhD; ${ }^{3}$ Anthony Santarelli, PhD; ${ }^{4}$ Edgardo Rivera, MD; ${ }^{3} \mathrm{John}$ Ashurst, DO

${ }^{1}$ Pacific Northwest University of Health Sciences College of Osteopathic Medicine (PNWU-COM); ${ }^{2}$ Department of Research, Kingman Regional Medical Center, W.L. Nugent 
Cancer Center; ${ }^{3}$ Department of Graduate Medical Education, Kingman Regional Medical Center; ${ }^{4}$ Department of Graduate Medical Education, Kingman Regional Medical Center, W.L. Nugent Cancer Center

Context: Though remaining the 2nd leading cause of death in the United States, non-specific tumorigenesis has been decreasing over the past 20 years. ${ }^{1,2}$ When assessed by geographical location it is apparent that the incidence of cancer diagnosis is reducing more quickly in urban settings than rural communities. ${ }^{3,4}$ However, reliable data from rural areas is sparse and likely idiosyncratic to the community of origin

Objective: To determine the incidence of cancer diagnosis of single organ systems within a rural southwest community.

Methods: A retrospective chart review of all patients diagnosed with a cancerous mass from 2004-2019 was conducted. Abstracted data included the patient sex, site of diagnosis (gastrointestinal, breast, bone, brain, cutaneous, gynecologic, head and neck, lung, pancreas, and urological), and major histopathology. Rates of new diagnosis by site and patient sex were compared to one another using repeated measures linear regression.

Results: The overall cancer incidence linearly increased ( $\mathrm{r} 2=0.900$ ) among both men and women by $372 \%$ from 2004 to $2019(\mathrm{p}<0.001)$. Rates of new diagnosis for cancer of the lung (339.6\% increase; 48(2004) vs 163(2019); $\mathrm{p}=0.010$ ) and breast (350.0\% increase; 10(2004) vs 35(2019); $\mathrm{p}=0.034$ ) showed the steepest incline. The most common histopathology observed in 2004 was squamous cell carcinoma $(25 \%$; 12/48), whereas the most common in 2019 was adenocarcinoma $(31 \%$; $51 / 163)$. No significant differences were detected between the sexes for the total new diagnoses across or within system.

Conclusion: Despite national trends showing a reduction in the number of new cancer patients per year, rates of diagnosis in the rural southwest are increasing. However, the mechanism producing this geographical divide remains unclear but may be due to changes in diagnostic screening or increases in patient access to care centers. Further research is needed to elucidate the factors driving increased rates of cancer diagnosis in rural America.

\section{References}

1. Siegel RL, Miller KD, Jemal A. Cancer statistics, 2020. CA Cancer J Clin. 2020 Jan;70(1):7-30. doi: 10.3322/caac.21590. Epub 2020 Jan 8. PMID: 31912902.

2. Meza R, Meernik C, Jeon J, Cote ML. Lung cancer incidence trends by gender, race and histology in the United States, 1973-2010.
PLoS One. 2015 Mar 30;10(3):e0121323. doi: 10.1371/journal. pone.0121323. PMID: 25822850; PMCID: PMC4379166.

3. Zahnd WE, James AS, Jenkins WD, Izadi SR, Fogleman AJ, Steward DE, Colditz GA, Brard L. Rural-Urban Differences in Cancer Incidence and Trends in the United States. Cancer Epidemiol Biomarkers Prev. 2018 Nov;27(11):1265-1274. doi: 10.1158/10559965.EPI-17-0430. Epub 2017 Jul 27. PMID: 28751476; PMCID: PMC5787045.

4. Meza R, Meernik C, Jeon J, Cote ML. Lung cancer incidence trends by gender, race and histology in the United States, 1973-2010. PLoS One. 2015 Mar 30;10(3):e0121323. doi: 10.1371/journal. pone.0121323. PMID: 25822850; PMCID: PMC4379166.

Financial Disclosures: None reported.

Support: None reported.

Ethical Approval: KRMC-0230. All procedures were approved by the Kingman Regional Medical Center Institutional Review Board.

Informed Consent: This study was conducted as a retrospective chart review of existing medical records.

^Poster No.: ${ }^{\star} \mathrm{H} 5$
Abstract No.: 82
Category: Health Services
Research Focus Area: Chronic Diseases \& Conditions
Medication Usage in African
American Inflammatory Bowel
Disease (IBD) Patients

${ }^{1}$ Landen Shane Burstiner, OMS-IV; ${ }^{2}$ Anna Owings, DO; ${ }^{2}$ Amor Royer, MD; ${ }^{3}$ Hannah Laird, MS-III; ${ }^{3}$ Jeshanah Johnson, MS-IV; ${ }^{3}$ Yousef Hreish, MS-IV; ${ }^{3}$ Madelyn Barr, MS-IV; ${ }^{4}$ Julia Liu, MD; ${ }^{5}$ Sarah Glover, DO

${ }^{1}$ Nova Southeastern University Dr. Kiran C. Patel College of Osteopathic Medicine (NSU-KPCOM); ${ }^{2}$ Department of Internal Medicine, University of Mississippi Medical Center; ${ }^{3}$ School of Medicine, University of Mississippi; ${ }^{4} \mathrm{Chief}$ of Gastroenterology, Morehouse School of Medicine; ${ }^{5} \mathrm{Chief}$ of Gastroenterology, University of Mississippi Medical Center

Context: Aminosalicylates, immunomodulators, corticosteroids (CS), and biological agents are the main classes of therapies used to treat IBD patients. The pattern of use for these agents amongst minority IBD populations is not well documented, especially the use of biologic treatment in African Americans (AAs).

Objective: To determine if any differences exist in the type and/or quantity of medications used among AA vs Caucasian American (CA) populations in the treatment of IBD. 
Methods: This was a retrospective chart review conducted using electronic health records of CA and AA patients with at least two ICD10 codes for Crohn's Disease (CD) or Ulcerative Colitis (UC) from 2012 to 2020 at a university-based tertiary center. Patients were excluded if they had incomplete health records and/or missing demographic information. Patient data was exported from Epic EMR software and de-identified prior to analysis. The charts of 100 randomly selected patients were manually checked to verify accuracy of exported data. Estimated income is based on Zip Code using 2013-2018 ACV Median Household Income. Prescriptions were extracted using both the generic and brand names of specific medications. Comparisons between groups were made using the Student's t-test, one way ANOVA, or Chi-squared test. Multivariate logistic regression models were adjusted for covariates on medication.

Results: 3049 patients met the inclusion criteria. A higher proportion of AA patients received prednisone $(p=0.041)$, while a higher proportion of CA patients received budesonide $(\mathrm{p}=0.020)$. Moreover, AAs had higher CS prescriptions per patient than CAs (1.81 vs 1.41 , respectively, $\mathrm{p}=0.006)$. Other than corticosteroids, there was also a significant difference in the proportion of African Americans who were prescribed aminosalicylates. There were no differences in the percentage of AA and CA patients on immunomodulators or biologics.

History of any IBD complications was positively associated with the prescription of CS ( $\mathrm{p}<0.001)$. Estimated income was not significantly associated with the prescription of prednisone, but it was positively associated with the prescription of budesonide $(\mathrm{p}=0.002)$. Of the covariates, only age, sub-type of IBD, and history of IBD complications were significantly associated (all $\mathrm{p}<0.001$ ) with the receipt of biologics. Race, sex, BMI, and estimated income were other covariates that were included in the model, but were not associated.

Conclusion: AA patients and patients at a lower income have similar access to biologic treatments, but are limited in their access to budesonide. AA patients receive significantly more CS prescriptions on average. Further studies are necessary to elucidate the reason behind the increased amount of CS prescriptions in AA patients, disease may be more severe in AA patients as indicated by the higher prevalence and amount of IBD-related complications, or CS may be less effective in the AA population, among other explanations. The finding that AAs are prescribed aminosalicylates less frequently is interesting. One explanation could be that AAs may have more severe disease (on average) at presentation, which may necessitate skipping over first line medications like aminosalicylates and proceeding straight to second or third line medications. Our study did not reveal any significant association between estimated income and access to biologic medications. However, additional studies examining the lowest income quartile or patients below poverty level may uncover limitations to care.

References None

Financial Disclosures: None Reported.

Support: None reported.

Ethical Approval: Study was reviewed and approved.

IRB File \#2019-0194

Health Outcomes of Inflammatory Bowel Disease (IBD) Subjects

Informed Consent: N/A 